\title{
EFEITO DA DEFORMAÇÃO POR CORTANTE NO CÁLCULO DE EDIFÍCIOS DE ANDARES MÚLTIPLOS COM NÚCLEOS ESTRUTURAIS
}

Ivan Francisco Ruiz Torres

Dissertação apresentada à Escola de Engenharia de São Carlos da Universidade de São Paulo, como parte dos requisitos para obtenção do título de Mestre em Engenharia de Estruturas

ORIENTADOR : Prof. Dr. Dagoberto Dario Mori

São Carlos

1999 


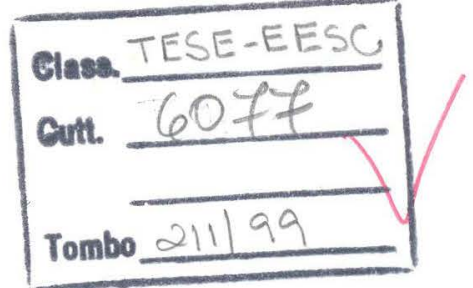

Ficha catalográfica preparada pela Seção de Tratamento da Informação do Serviço de Biblioteca - EESC/USP

Dissertação (Mestrado) -- Escola de Engenharia de São Carlos-Universidade de São Paulo, 1999.

Área: Engenharia de Estruturas.

Orientador: Prof. Dr. Dagoberto Dario Mori.

1. Núcleo. 2. Deformação por cortante. 3. Fator de forma. 4. Viga de Timoshenko. I. Título. 
FOLHA DE APROVACÃO

Candidato: Engenheiro IVAN FRANCISCO RUIZ TORRES

Dissertação defendida e aprovada em 07-05-1999

pela Comissão Julgadora:

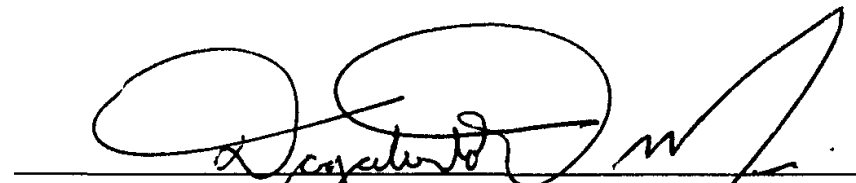

Prof. Doutor DAGOBERTO DARIO MORI (Orientador)

(Escola de Engenbaria de São Carlos - Universidade de São Paulo)

Lemo lotect xe $C$.

Prof. Doutor MARCIO ROBERTO SILVA CORRÊA

(Escola de Engenharia de São Carlos - Universidade de São Paulo)

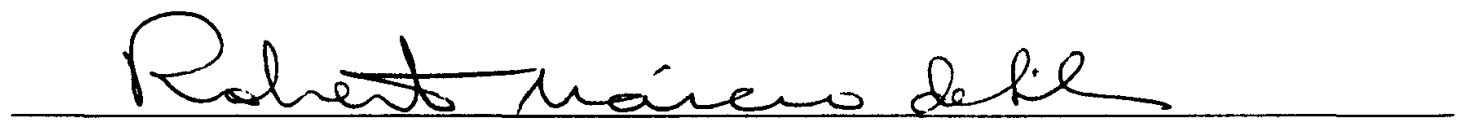

Prof. Doutor ROBERTO MARCIO DA SILVA

(Universidade Federal de Minas Gerais - UFMG)

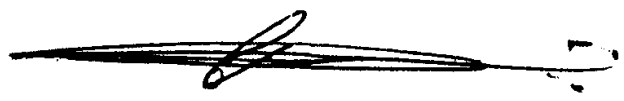

Prof. Titular CARLITO CALIL JUNIOR

Coordenador da Área de Engenharia de Estruturas

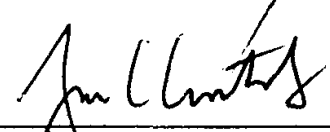

JOSÉ CARLOS A. CINTRA

Presidente da Comısşo de Pós-Graduação da EESC 
Ao Professor Dagoberto Dario Mori, pela atenção e excelente orientação dadas ao longo da elaboração deste trabalho.

À Coordenadoria de Aperfeiçoamento de Pessoal de Nível Superior - CAPES, pela bolsa de estudo concedida.

Aos professores e funcionários do Departamento de Estruturas da EESC-USP que de alguma forma colaboraram para a realização desta pesquisa.

Aos funcionários da Biblioteca Central da EESC-USP, pelo auxílio na realização da pesquisa bibliográfica desta dissertação.

A todos os colegas que dispuseram de parte de seu tempo e de seus conhecimentos para me auxiliar na elaboração deste trabalho, especialmente os doutorandos Ângelo Vieira Mendonça e Carlos Humberto Martins. 


\section{SUMÁRIO}

LISTA DE FIGURAS

LISTA DE TABELAS

RESUMO

ABSTRACT

1 - INTRODUÇÃO ......................................................................... 1

1.1 - Considerações gerais ................................................................... 1

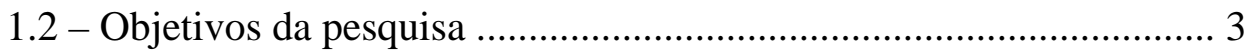

1.3 - Justificativa da pesquisa .......................................................... 5

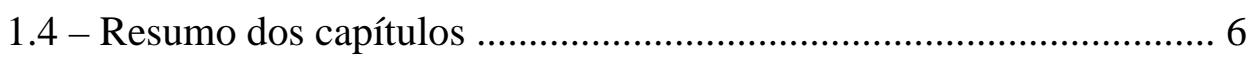

2 - REVISÃO BIBLIOGRÁFICA ....................................................... 8

2.1 - Dissertações e Teses realizadas no Departamento de Estruturas da EESC - USP ........................................................ 13

3 - TEORIAS DE FLEXÃO DE BARRAS ........................................... 17

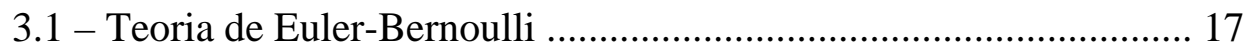

3.2 - Teoria de Timoshenko ............................................................ 19

3.3 - Teoria de alta-ordem .............................................................. 21

4 - MÉTODO SEMI-INVERSO NA FLEXÃO DE BARRAS -

APLICAÇÃO À TEORIA DE TIMOSHENKO....................................26

4.1 - Tensão de cisalhamento na seção transversal de uma barra suposição da Resistência dos Materiais .........................................26

4.2 - Método semi-inverso aplicado à flexão de barras - cálculo de fatores de forma e da distribuição das tensões de cisalhamento na seção transversal ................................................. 29

4.2.1 - Dedução da expressão do fator de forma ........................... 34

4.3 - Formulação em elementos finitos para cálculo da função de

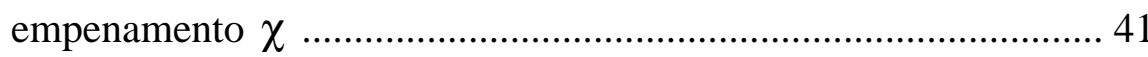

4.3.1 - Elemento quadrangular isoparamétrico ...............................44 
4.4 - Elementos de Timoshenko e respectivas matrizes de rigidez 55

5 - TEORIA DE TIMOSHENKO APLICADA AOS ELEMENTOS

VERTICAIS DE CONTRAVENTAMENTO_...................................... 58

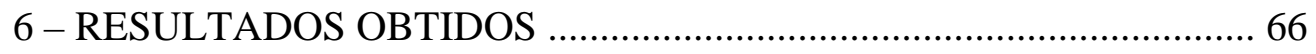

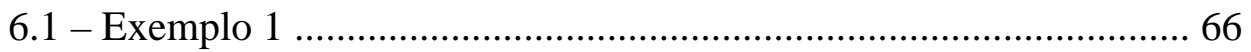

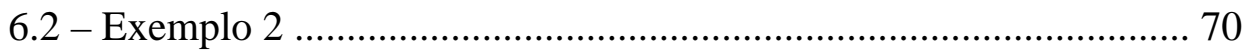

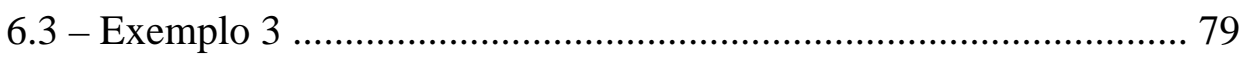

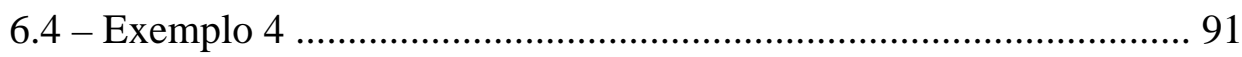

6.5 - Exemplo 5 .......................................................................... 101

7 - FATORES DE FORMA DE PERFIS ESTRUTURAIS DE AÇO

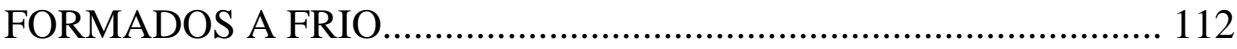

7.1 - Tabelas com o valor dos fatores de forma dos perfis formados a frio

7.2 - Exemplos de aplicação dos fatores de forma ................................ 121

8 - CONSIDERAÇÕES FINAIS E SUGESTÕES................................... 124

REFERÊNCIAS BIBLIOGRÁFICAS............................................ 126

BIBLIOGRAFIA COMPLEMENTAR …........................................ 131 


\section{LISTA DE FIGURAS}

Figura 1 - Sistema de coordenadas (formulação das teorias de Euler-Bernoulli

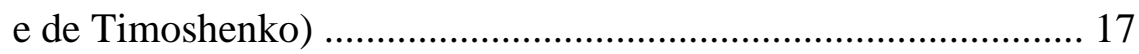

Figura 2 - Flexão pela teoria de Euler-Bernoulli ........................................ 18

Figura 3 - Rotação da seção transversal (teoria de Euler-Bernoulli) .......... 19

Figura 4 - Parâmetros nodais (teoria de Euler-Bernoulli) ........................... 19

Figura 5 - Rotação da seção transversal (teoria de Timoshenko) ............... 20

Figura 6 - Sistema de coordenadas (formulação da teoria de

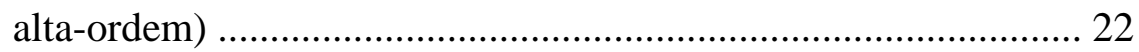

Figura 7 - Corte de uma fatia de viga …..................................................... 27

Figura 8 - Equilíbrio da fatia de viga, em vista lateral .............................. 27

Figura 9 - Convenção de forças transversais e momentos

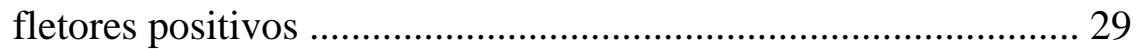

Figura 10 - Seção deformada e seu plano médio de rotação .......................... 33

Figura 11 - Força transversal com componentes nas direções y e z ............. 36

Figura 12 - Elemento quadrangular isoparamétrico .................................... 44

Figura 13 - "Elemento escravo" ............................................................... 45

Figura 14 - Transformação de elementos bidimensionais em

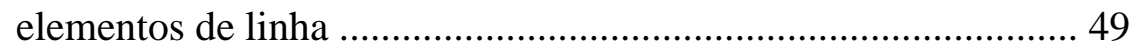

Figura 15 - Definição de parâmetros no elemento de linha ........................... 50

Figura 16 - Sistema de coordenadas - matriz de ARCHER …...................... 56

Figura 17 - Sistema de coordenadas - Matriz de PILKEY et al. .................. 56

Figura 18 - Coordenadas de deslocamento do ELM - 01 ........................... 58

Figura 19 - Coordenadas de deslocamento do ELM - 02 ........................... 59

Figura 20 - Coordenadas de deslocamento do ELM - 03 ........................... 60

Figura 21 - Coordenadas de deslocamento do ELM - 04 ............................ 65 
Figura 22 - Coordenadas de deslocamento do ELM - 05

Figura 23 - Viga engastada com carga concentrada na extremidade ............ 66

Figura 24 - Seção transversal da viga do exemplo 1 .................................. 67

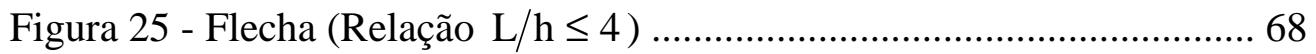

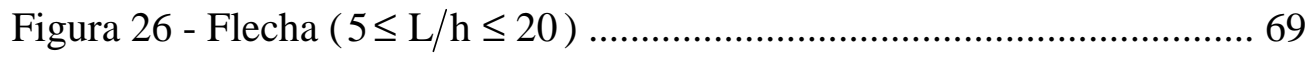

Figura 27 - Corte transversal da estrutura do exemplo 2 ........................... 70

Figura 28 - Equilíbrio à torção do pavimento .............................................75

Figura 29 - Rotação em torno de $X_{g}$ no topo dos pavimentos .................... 77

Figura 30 - Momento torçor no núcleo $\mathrm{N}_{1}$............................................ 77

Figura 31 - Esforço cortante na direção do eixo z local do

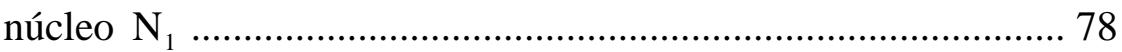

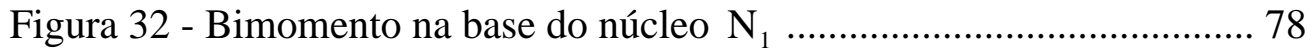

Figura 33 - Planta do pavimento-tipo do exemplo 3 ................................... 79

Figura 34 - Translação em $Y_{g}$ do nó mestre das lajes ................................. 81

Figura 35 - Rotação das lajes em torno de $X_{\mathrm{g}}$ …................................... 82

Figura 36 - Momento fletor em torno do eixo $\mathrm{z}$ na base do pilar 3 ............. 83

Figura 37 - Momento fletor em torno do eixo y local na base do

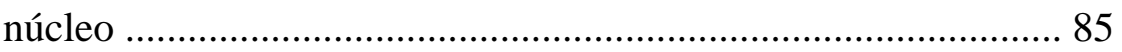

Figura 38 - Esforço cortante na direção do eixo z local do núcleo ................ 86

Figura 39 - Somatória de esforços cortantes nos pilares na

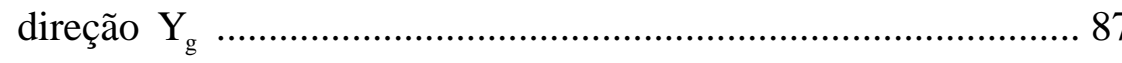

Figura 40 - Esforço cortante no pilar 1 na direção do eixo y local ............... 88

Figura 41 - Bimomento na extremidade inferior do núcleo ........................ 89

Figura 42 - Planta do pavimento-tipo do exemplo 4 .................................... 92

Figura 43 - Translação do nó mestre da laje na direção $Z_{\mathrm{g}}$...................... 93

Figura 44 - Proporção do esforço cortante na direção $Z_{\mathrm{g}}$ absorvido pelo núcleo em relação ao total do pavimento .................................. 95

Figura 45 - Esforço cortante na direção do eixo z local do pilar 2 ............... 96

Figura 46 - Momento fletor em torno do eixo y local no topo do 
pilar 2 97

Figura 47 - Esforço cortante na direção do eixo z local do pilar 8 98

Figura 48 - Momento fletor em torno do eixo y local no topo do pilar 8 99

Figura 49 - Planta do pavimento-tipo do exemplo 5 102

Figura 50 - Translação do nó mestre da laje na direção do eixo $Z_{g}$ 104

Figura 51 - Rotação das lajes em torno do eixo $\mathrm{X}$ 105

Figura 52 - Esforço cortante no pilar 20 na direção de $Z_{g}$........................... 108

Figura 53 - Momento fletor em torno de y na base do pilar 7 .................... 109

Figura 54 - Momento torçor no núcleo $\mathrm{N}_{1}$................................................. 110

Figura 55 - Perfil U simples ............................................................... 112

Figura 56 - Perfil U enrijecido ……………………………………..... 112

Figura 57 - Perfil Z simples .................................................................... 113

Figura 58 - Perfil Z enrijecido ............................................................... 114

Figura 59 - Perfil cartola …………………………………………...... 114

Figura 60 - Viga de aço submetida a força uniformemente distribuída ...... 121

Figura 61 - Viga de aço submetida a força concentrada na

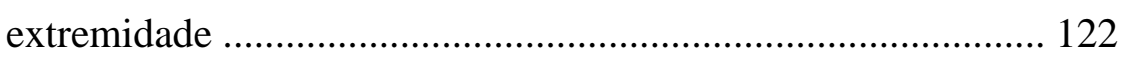

Figura 62 - Seção transversal do perfil Z simples ......................................... 123 


\section{LISTA DE TABELAS}

Tabela 1 - Sumário de elementos de Timoshenko apresentados

Tabela 2 - Deslocamentos segundo as teorias de Euler-Bernoulli, de Timoshenko, de Alta-ordem e da Elasticidade

Tabela 3 - Rotação em torno do eixo $X_{g}$ no topo dos pavimentos ............. 72

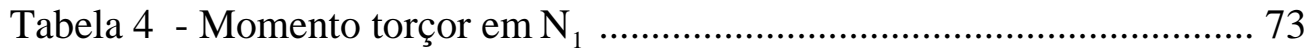

Tabela 5 - Esforço cortante no núcleo $\mathrm{N}_{1}$ na direção de seu eixo $\mathrm{z}$ local 74

Tabela 6 - Bimomento no núcleo $\mathrm{N}_{1}$ (ou no núcleo $\mathrm{N}_{2}$ ) 76

Tabela 7 - Translação do nó mestre das lajes na direção de $\mathrm{Y}_{\mathrm{g}}$ 81

Tabela 8 - Rotação em torno do eixo vertical $X_{g}$ 82

Tabela 9 - Momento fletor em torno do eixo z local do pilar 3, em sua extremidade inferior

Tabela 10 - Momento fletor em torno do eixo z local do pilar 2, em sua extremidade inferior

Tabela 11 - Momento fletor em torno do eixo y local do núcleo, na extremidade inferior

Tabela 12 - Esforço cortante na direção do eixo z local do núcleo 85

Tabela 13 - Somatória de esforços cortantes nos pilares na direção $Y_{g}$...... 86

Tabela 14 - Esforço cortante no pilar 1 na direção y local ......................... 87

Tabela 15 - Bimomento na extremidade inferior do núcleo ......................... 88

Tabela 16 - Translação do nó mestre da laje na direção de $Z_{\mathrm{g}}$.................... 93

Tabela 17 - Distribuição entre o núcleo e os pilares do esforço cortante na direção do eixo $Z_{\mathrm{g}}$ sem considerar deformação por cortante 
Tabela 18 - Distribuição entre o núcleo e os pilares do esforço cortante na direção do eixo $Z_{\mathrm{g}}$ considerando deformação

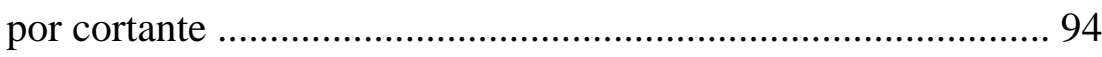

Tabela 19 - Momento fletor em torno do eixo z local do núcleo ................ 95

Tabela 20 - Esforço cortante na direção do eixo z local do pilar 2 .............. 96

Tabela 21 - Momento fletor em torno do eixo y local do pilar 2 ................ 97

Tabela 22 - Esforço cortante na direção do eixo z local do pilar 8 .............. 98

Tabela 23 - Momento fletor em torno do eixo y local do pilar 8 ................ 99

Tabela 24 - Dimensões dos pilares .......................................................... 101

Tabela 25 - Dimensões das vigas ......................................................... 101

Tabela 26 - Carregamento aplicado à estrutura ....................................... 103

Tabela 27 - Translação $(\mathrm{cm})$ do nó mestre da laje na direção de $Z_{\mathrm{g}}$......... 104

Tabela 28 - Rotação das lajes em torno do eixo $\mathrm{X}_{\mathrm{g}}\left(10^{-3} \mathrm{rad}\right)$................... 105

Tabela 29 - Soma dos esforços cortantes nos núcleos $\mathrm{N}_{1}$ e $\mathrm{N}_{2}$ na direção do

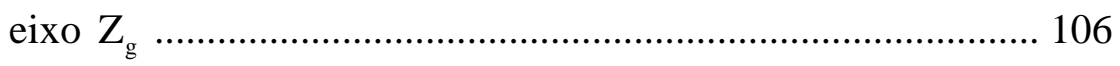

Tabela 30 - Soma dos esforços cortantes em todos os pilares na direção do

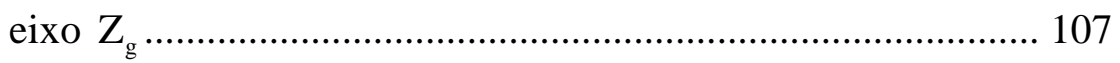

Tabela 31 - Esforço cortante $(\mathrm{kN})$ na direção do eixo $Z_{\mathrm{g}}$ no pilar 20 ........ 108

Tabela 32 - Momento fletor (kN.cm) em torno do eixo y local do pilar 7 (paralelo ao eixo $\mathrm{Y}_{\mathrm{g}}$ ), na extremidade inferior dos elementos 109

Tabela 33 - Momento torçor $(\mathrm{kN} . \mathrm{cm})$ no núcleo $\mathrm{N}_{1}$

Tabela 34 - Características geométricas da seção transversal dos perfis U simples

Tabela 35 - Características geométricas da seção transversal dos perfis U enrijecidos

Tabela 36 - Características geométricas da seção transversal dos perfis Z simples

Tabela 37 - Características geométricas da seção transversal dos perfis Z 
enrijecidos

Tabela 38 - Características geométricas da seção transversal dos perfis cartola 


\section{RESUMO}

TORRES, I. F. R. (1999). Efeito da deformação por cortante no cálculo de edifícios de andares múltiplos com núcleos estruturais. São Carlos, 1999. 131p. Dissertação (Mestrado) - Escola de Engenharia de São Carlos, Universidade de São Paulo.

O principal objetivo deste trabalho é realizar a análise estrutural de edifícios de andares múltiplos que apresentam núcleos resistentes, considerando a deformação pelo esforço cortante nos mesmos, bem como nos pilares. Para atingir esse objetivo, será preciso que o comportamento à flexão dos elementos verticais de contraventamento passe a ser regido pela teoria de barras de Timoshenko e não mais pela de Euler-Bernoulli. Foram então desenvolvidos algoritmos que, utilizando o Método dos Elementos Finitos (MEF), permitem calcular os fatores de forma de quaisquer seções transversais abertas de paredes delgadas pertencentes a núcleos estruturais, bem como a distribuição da tensão de cisalhamento na seção transversal em função do esforço cortante atuante. As alterações acima descritas foram feitas em um programa de análise de edifícios denominado CEASO 01, de autoria de MATIAS JR (1997). Embora esse programa realize análise não-linear geométrica, a consideração da deformação por cortante só foi implementada na análise linear. Apresentam-se, ao final, exemplos numéricos que permitem avaliar a influência da deformação pelo esforço cortante sobre os deslocamentos e esforços de núcleos resistentes e pilares

Palavras-chave : Núcleo; deformação por cortante; fator de forma; viga de Timoshenko. 


\begin{abstract}
TORRES, I. F. R. (1999). Effect of shear deformation in the analysis of multistory buildings with structural cores. São Carlos, 1999. 131p. Dissertação (Mestrado) Escola de Engenharia de São Carlos, Universidade de São Paulo.
\end{abstract}

The main aim of this work is to perform structural analysis of multistory buildings with resistant cores, taking into account shear deformation in those elements, as well as in columns. To achieve this objective, the flexural behaviour of vertical elements must be governed by Timoshenko beam theory, rather than the Euler-Bernoulli theory. Procedures using the finite element method (FEM) were developped, which enable to evaluate shear correction factors of generic thin-walled open sections and shear stress distribution as a function of the shear resultant. Changes described above were made in a structural analysis program named CEASO 01, whose author is MATIAS JR (1997). Even though this program is able to perform nonlinear analysis, only in linear analysis the effect of shear deformation is taken into account. Numerical examples are provided, which enable to evaluate the influence of taking into account shear deformation on displacements and stress resultants of resistant cores and columns.

keywords : core; shear deformation; shear correction factor; Timoshenko beam. 


\section{1 - INTRODUÇÃO}

\section{1 - Considerações gerais}

Entre os diversos sistemas estruturais projetados para permitir o contraventamento de estruturas de edifícios altos, podem-se destacar:

- Pórticos planos: constituídos pela associação de pilares, vigas e eventualmente elementos diagonais, ligados entre si por nós rígidos ou semi-rígidos e apresentando flexão num único plano.

- Painéis treliçados: também constituídos por pilares, vigas e barras diagonais, ligados por nós perfeitamente articulados. Esse sistema é muito utilizado em edifícios de estrutura metálica.

- Painéis-parede: constituídos por pilares-parede, que são aqueles em que uma das dimensões é consideravelmente maior que a outra. Quando dois ou mais pilares-parede estão associados tridimensionalmente, assumem comportamento estrutural semelhante ao de núcleos resistentes.

- Sistema tubular: neste sistema, os pórticos são colocados principalmente no contorno do edifício, formando uma estrutura assemelhada a um tubo. Os pilares que integram os pórticos são posicionados bastante próximos entre si e as vigas apresentam grande altura. $\mathrm{O}$ objetivo é aumentar a rigidez da estrutura à flexão.

- Núcleos resistentes: são formados pela associação tridimensional de pilares-parede, vindo a formar, num corte horizontal, uma seção transversal aberta de paredes delgadas. Podem ser parcialmente fechados por vigas no nível das lajes (lintéis). Devido à sua geometria, são usualmente modelados como barras de seção transversal aberta, às quais se aplica a teoria de flexo-torção de VLASSOV (1961).

Deve-se ressaltar que na análise de estruturas de edifícios altos pode-se admitir que a laje seja infinitamente rígida em seu próprio plano, o que faz com que ela atue como um elemento de compatibilização das translações no plano horizontal e das rotações em torno do eixo vertical das extremidades dos pilares e dos núcleos 
resistentes que incidem nela. Assim, define-se um ponto no plano da laje que irá concentrar a rigidez à translação de todos os elementos verticais que nela incidem, bem como a contribuição por parte de todos esses elementos à rigidez da laje à torção. Esse ponto é denominado nó mestre da laje. Se a laje apresentar regiões vazadas ou dimensões muito grandes, deve-se estudar a conveniência de analisá-la como casca, ou seja, além da flexão (comportamento de placa), a laje apresentará deformações no seu próprio plano (comportamento de chapa).

$\mathrm{Na}$ análise estrutural de edifícios altos, o método mais utilizado é o dos deslocamentos, com o emprego de técnicas de análise matricial.

Quanto à análise estrutural de núcleos resistentes, existem basicamente dois métodos: o que utiliza a técnica do meio contínuo e o que utiliza os chamados métodos discretos, sendo o principal destes o método dos deslocamentos.

Pela técnica do meio contínuo, o comportamento do núcleo é definido por uma equação diferencial ou por um sistema delas, que são resolvidas por integração direta ou por algum método numérico. A solução das equações diferenciais fornece os deslocamentos e esforços, ao longo do comprimento da estrutura. Quando se utiliza este método, o núcleo resistente é analisado como uma barra cujo comportamento à torção segue a teoria de flexo-torção de Vlassov. Esse método leva geralmente a soluções simples, mas tem o inconveniente de não permitir que as características geométricas do núcleo variem ao longo de sua altura.

Já o tratamento discreto consiste na subdivisão da estrutura em um certo número de elementos, cuja interação se dá através de pontos nodais. No método dos deslocamentos, transfere-se para os nós a rigidez dos elementos que a eles se ligam, sendo que a cada um desses nós estão associados parâmetros de deslocamento. Mediante resolução de um sistema linear, calculam-se os deslocamentos nodais que permitirão calcular, utilizando as matrizes de rigidez de cada elemento, os esforços em cada divisão do núcleo. Este é um processo mais versátil do que a técnica do meio contínuo, por permitir variação de características da estrutura ao longo de sua altura.

Quando se utiliza o método dos deslocamentos, são adotados basicamente dois modelos para o núcleo resistente: 
- O que se baseia na teoria de flexo-torção de Vlassov, em que o conjunto de paredes delgadas forma um único elemento de barra, o qual terá uma coordenada adicional, a primeira derivada da rotação, associada a um esforço adicional, o bimomento. A matriz de rigidez e o vetor de forças nodais desse elemento foram desenvolvidos por TARANATH (1968).

- O que considera o núcleo como uma associação tridimensional de paredes planas sendo que cada parede, no intervalo de duas lajes, é substituída por um pilar de rigidez equivalente, com duas vigas de rigidez infinita à flexão nas extremidades. Consideram-se apenas forças de cisalhamento como interação entre esses pilares.

Sendo o modelo adotado para o núcleo resistente, neste trabalho, o de Taranath, o estudo da consideração da deformação por esforço cortante em edifícios altos com núcleos resistentes requer uma análise das principais teorias de flexão de barras, de modo a considerar o efeito acima citado.

\section{2 - Objetivos da pesquisa}

O objetivo desta pesquisa é incluir a consideração do efeito da deformação por esforço cortante na análise de estruturas de edifícios altos com núcleos resistentes, de modo a retratar de forma mais precisa o seu comportamento estrutural e atender com maior eficiência aos requisitos de economia e segurança.

Esse efeito foi considerado por meio de alterações no programa de análise estrutural de edifícios altos CEASO 01, de autoria de MATIAS JR. (1997). A sigla CEASO vem de Cálculo de Edifícios Altos em teoria de Segunda Ordem, programa desenvolvido por MORI (1992) e que serviu de base para o programa CEASO 01. Este último analisa a interação entre núcleos resistentes e pórticos planos ou tridimensionais, mediante a definição de cinco tipos de elementos verticais (o quinto é o elemento de núcleo), de dois tipos de elementos horizontais (vigas) e de dois tipos de elementos diagonais, sendo todos estes elementos de barra. Admite-se que a laje seja indeformável em seu plano, sendo portanto um elemento compatibilizador de translações e da rotação em torno do eixo longitudinal dos elementos verticais. $\mathrm{O}$ programa permite a análise em teoria de primeira e de segunda ordens, sendo que a montagem da matriz de rigidez do núcleo em segunda ordem é feita segundo o 
processo desenvolvido por MORI (1992), que utiliza técnicas numéricas auxiliadas pelas sub-rotinas ODEPACK* desenvolvidas por HINDMARCH (1983).

Em CEASO 01, a flexão tanto dos núcleos estruturais quanto dos pilares em torno dos eixos y e z (sendo yz o plano horizontal) é regida pela teoria de EulerBernoulli, na qual a distorção da seção transversal da barra é desprezada e considerase, portanto, que o deslocamento transversal do eixo da barra seja relacionado apenas à flexão.

No presente trabalho, foi adotada, para a flexão dos elementos verticais de contraventamento, a teoria de Timoshenko, que leva em conta a influência da deformação pelo cisalhamento no plano da seção transversal.

As alterações no programa CEASO 01, que possibilitaram a análise de núcleos e pilares pela teoria de Timoshenko, consistiram basicamente no acréscimo de sub-rotinas (e sua adaptação ao programa original) com as seguintes funções:

- Cálculo dos fatores de forma de qualquer seção transversal aberta de paredes delgadas. Estes fatores são necessários à montagem da matriz de rigidez dos núcleos resistentes.

- Cálculo das matrizes de rigidez dos elementos de núcleo e dos demais tipos de elementos verticais com a consideração da deformação pelo esforço cortante.

- Cálculo das tensões de cisalhamento em qualquer ponto da seção transversal do núcleo, em função do esforço cortante atuante. Com a distribuição de tensões obtida, calcula-se a posição do centro de torção do núcleo. Esse cálculo será mais preciso do que aquele realizado com base na suposição de que a tensão de cisalhamento seja constante ao longo da espessura das paredes.

As alterações na entrada de dados foram mínimas: é preciso optar por considerar ou não a deformação por cortante (havendo a possibilidade de considerála separadamente nos núcleos e nos pilares); é preciso informar se os fatores de forma serão calculados pelo processo geométrico ou de energia e deve-se também informar os fatores de forma das seções dos pilares, quando estas não forem retangulares. Se a seção for retangular, o programa irá calculá-los.

\footnotetext{
* Conjunto de sub-rotinas para resolução de sistemas de equações diferenciais ordinárias.
} 
As alterações no CEASO 01 que possibilitam a análise dos elementos verticais com a admissão de deformação pelo cisalhamento devido ao cortante só estão adaptadas à análise em teoria de primeira ordem.

Dispondo-se do CEASO 01 modificado, pôde-se realizar a análise estrutural de alguns exemplos e verificar a influência que as alterações descritas acima tiveram sobre os resultados obtidos.

\section{3 - Justificativa da pesquisa}

A grande maioria dos trabalhos na área de edifícios altos desconsidera a deformação pelo esforço cortante na análise dos elementos de barra (vigas e pilares), pois sabe-se que esse esforço só passa a ter maior influência no comportamento desses elementos quando a relação entre seu comprimento (L) e a altura da seção transversal ( $\mathrm{h}$ ) torna-se reduzida. Mais exatamente, o parâmetro que indica o maior ou menor efeito do esforço cortante é $\mathrm{g}=12 \cdot \mathrm{E} \cdot \mathrm{I} \cdot \alpha /\left(\mathrm{G} \cdot \mathrm{A} \cdot \mathrm{l}^{2}\right)$, em que E, I, $\alpha, G, A$, e 1 são, respectivamente o módulo de elasticidade longitudinal do material, o momento de inércia em relação ao eixo em torno do qual se dá a flexão, o fator de forma na direção do carregamento, o módulo de elasticidade transversal, a área da seção transversal e o comprimento da barra.

Em relação aos núcleos estruturais, sabe-se que, por sua própria geometria, apresentam razão entre momento de inércia e área da seção transversal maior que a de seções transversais cheias. Além disso, o fator de forma de seções de paredes delgadas tende a ter valor substancialmente mais elevado do que o das seções cheias, como será visto no capítulo 6 (Resultados Obtidos). Esses dois fatores fazem com que o efeito do esforço cortante em núcleos resistentes seja mais significativo do que em pilares de dimensões usuais.

No caso de edifícios com núcleos resistentes, o comportamento da estrutura à flexão devida à carga de vento será fortemente influenciado pelo comportamento destes elementos, uma vez que eles absorvem uma parcela muito grande do carregamento horizontal. Como decorrência, pode-se prever em edifícios com núcleos estruturais que o efeito da deformação por esforço cortante será bem maior 
do que em edifícios cuja estrutura de contraventamento seja constituída exclusivamente por pórticos planos ou tridimensionais e este trabalho objetiva oferecer uma avaliação desse efeito.

\section{4 - Resumo dos capítulos}

O capítulo 2 apresenta um breve resumo da evolução histórica das teorias de flexão de barras, desde o surgimento da teoria tradicional (Euler-Bernoulli) em 1705, passando pela exposição da teoria de TIMOSHENKO (1921), até se chegar à formulação da teoria de alta-ordem (1981).

Num item à parte são reunidas dissertações e teses realizadas no Departamento de Estruturas da Escola de Engenharia de São Carlos, da Universidade de São Paulo (EESC-USP) que analisam núcleos resistentes isoladamente ou edifícios cuja estrutura apresenta núcleos resistentes, indicando-se para cada um desses trabalhos: o método de análise empregado e o modelo adotado para o núcleo, se a análise inclui a interação dos núcleos com pórticos tridimensionais, se a análise é feita em teoria de primeira ou de segunda ordens e se é considerado o efeito da deformação por esforço cortante nas paredes do núcleo estrutural.

No capítulo 3, apresentam-se as hipóteses cinemáticas nas quais se baseiam as três teorias de flexão de barras, bem como as equações diferenciais que regem a flexão em cada uma delas.

No capítulo 4, apresenta-se inicialmente a dedução do equacionamento das tensões de cisalhamento, do empenamento $\chi$ e dos fatores de forma da seção transversal de uma barra pelo método semi-inverso.

Apresenta-se em seguida a formulação em elementos finitos para cálculo de tensões de cisalhamento e de fatores de forma tanto de seções cheias como de seções de paredes delgadas.

No final do capítulo, são apresentadas as duas matrizes de rigidez de elementos de barra de Timoshenko que foram utilizadas na alteração de CEASO 01. O cálculo dessas matrizes requer o conhecimento dos fatores de forma da seção transversal da barra. 
No capítulo 5, apresentam-se as coordenadas de deslocamento dos elementos verticais de contraventamento do programa CEASO 01 e as matrizes de rigidez desses elementos modificadas para que a distorção devida ao cortante fosse considerada.

O capítulo 6 é dedicado à análise de estruturas tomadas como exemplos, para verificar a influência de considerar a deformação por força cortante sobre os deslocamentos e esforços apresentados pela estrutura analisada. São ao todo cinco exemplos, sendo o primeiro utilizado para aferição das alterações feitas no programa CEASO 01. Os três exemplos seguintes foram extraídos de trabalhos de outros autores e o último criado por este autor de modo a possuir características que o tornassem representativo de edifícios projetados. Em cada exemplo, apresentam-se os resultados em tabelas e também graficamente.

No capítulo 7 são apresentadas tabelas de características geométricas de perfis de aço formados a frio, que são complementadas com o acréscimo dos valores dos fatores de forma de suas seções transversais. Com este capítulo, pretende-se oferecer como contribuição à análise de estruturas metálicas valores mais precisos de fatores de forma de seções de perfis de chapa dobrada do que os que são usualmente empregados, uma vez que se dispõe de um processo de cálculo mais refinado. Apresentam-se também dois exemplos numéricos, para demonstrar a utilização dos fatores de forma no cálculo de flechas em vigas.

No capítulo 8 são apresentadas conclusões que puderam ser extraídas da análise dos resultados obtidos no capítulo 6, assim como sugestões para trabalhos futuros que tratem deste tema.

Apresentam-se ao final as Referências Bibliográficas, com as obras e artigos que foram citados nesta dissertação, enquanto na Bibliografia Complementar são apresentadas obras com conteúdo relacionado ao trabalho desenvolvido. 


\section{2 - REVISÃO BIBLIOGRÁFICA}

A teoria tradicionalmente utilizada para a flexão de elementos de barra, conhecida como teoria de Euler-Bernoulli, ou teoria de engenharia, data de 1705. Neste ano, JAMES BERNOULLI estabeleceu que a resistência de uma barra fletida provém da extensão e contração das fibras longitudinais, além de definir a linha elástica como a posição deformada do eixo. Em 1742, DANIEL BERNOULLI sugere a EULER que a equação diferencial da linha elástica poderia ser encontrada através da minimização da integral do quadrado da curvatura ao longo do comprimento da barra.

Baseado nessa sugestão, EULER foi capaz de obter a equação diferencial da linha elástica e de classificar as várias formas que ela podia assumir. Em seu trabalho, a barra é entendida como uma linha de partículas que resistem à flexão.

Deve-se também destacar o trabalho de COULOMB, que foi o primeiro a tratar as barras como tendo uma seção finita, obtendo a posição da linha neutra ou eixo de equilíbrio e calculando corretamente o momento das forças elásticas. Também demonstrou que as tensões normais em uma barra fletida derivam unicamente da extensão e contração de suas fibras longitudinais.

A teoria de viga de Timoshenko, que considera a deformação pela tensão de cisalhamento, foi exposta inicialmente pelo autor em 1921. Em seu artigo, já se colocava a necessidade de um fator de forma, que corrigisse a suposição de tensão de cisalhamento constante ao longo da altura da viga adotada nessa teoria.

A partir da década de 50, com o desenvolvimento do método dos elementos finitos (MEF), iniciou-se o desenvolvimento de elementos de barra baseados na teoria de Timoshenko, sendo que os principais deles estão indicados a seguir: 
McCALLEY* apud TESSLER \& DONG (1981): realizou a subdivisão do deslocamento transversal $\mathrm{w}$ em duas parcelas - $\mathrm{w}_{\mathrm{b}}$ devido à flexão e $\mathrm{w}_{\mathrm{s}}$ devido ao cisalhamento. O elemento apresenta dois nós e tem como parâmetros nodais W $\left(\mathrm{w}_{\mathrm{b}}+\mathrm{w}_{\mathrm{s}}\right)$ e $\theta$ (definido como $\left.\mathrm{dw}_{\mathrm{b}} / \mathrm{dx}\right)$.

ARCHER (1965): utilizando a mesma formulação de McCalley, deduziu as matrizes de rigidez para vigas de seção transversal variável.

SEVERN (1970) e DAVIS et al. (1972): utilizaram abordagens levemente diferentes, mas chegaram a resultados equivalentes aos anteriores. Severn derivou a matriz de rigidez a partir das componentes de tensão usualmente assumidas no problema de flexão.

Entre os elementos cuja formulação se baseou em princípios variacionais, destacam-se o de CARNEGIE et al. (1969) e o de DONG \& WOLF (1973). Ambos desenvolveram elementos com mais de dois nós.

HUGHES et al. (1977): até o trabalho destes autores, o elemento de dois nós com aproximação linear tanto para o deslocamento transversal do centróide da seção transversal ( w ) como para a rotação da seção $(\theta)$ apresentava resultados razoáveis apenas para vigas em que a razão entre a altura da seção transversal e o comprimento da barra era elevada. Se utilizado para vigas em que o comportamento à flexão fosse dominante (vigas de maior comprimento e menor altura da seção), o elemento tornava-se cada vez mais rígido conforme aumentava a sua esbeltez, evidenciando um bloqueio da solução.

Este fenômeno, conhecido como shear locking, deriva do fato de que aproximações de mesmo grau para w e $\theta$ levam a que não se consiga satisfazer, no limite, a condição de distorção nula na seção transversal (condição de EulerBernoulli para vigas esbeltas).

Entre as opções para contornar o problema estão: utilizar polinômios interpoladores de graus distintos para o deslocamento e a rotação; adotar um campo de deformações impostas ao longo do comprimento da barra ou realizar, como proposto pelos autores, a chamada integração seletiva reduzida, que consiste na

\footnotetext{
* McCALLEY, R. B. (1963). Rotary inertia correction for mass matrices. General Electric Knolls Atomic Power Laboratory, Schenectady, New York (Report DIG/AS 63-73) apud TESSLER, A. ; DONG, S. B. (1981). On a hierarchy of conforming Timoshenko beam elements. Computers \& Structures v. 14, n. 3-4, p. 335-344.
} 
subintegração dos termos da matriz de rigidez referentes à energia de deformação por esforço cortante, integrando-se normalmente os termos relativos à deformação normal devida à flexão. Este procedimento permitiu que se eliminasse o shear locking e que se pudesse utilizar o elemento numa ampla gama de relações entre a altura da seção transversal e o comprimento da barra.

Entre os denominados elementos de ordem superior, assim chamados por envolverem a continuidade entre elementos de mais parâmetros nodais do que os exigidos pelos princípios variacionais, como, por exemplo, $\mathrm{dw} / \mathrm{dx}, \mathrm{d} \theta / \mathrm{dx}, \gamma$, etc, destacam-se os de KAPUR (1966), NICKEL \& SECOR (1972), THOMAS et al. (1973) e THOMAS \& ABBAS (1975).

Deve-se notar que estes quatro últimos elementos apresentam um número elevado de parâmetros nodais e interpolam deslocamentos e rotações de forma independente. Entretanto, como será visto posteriormente, a relação existente entre momento fletor e esforço cortante permite que se estabeleçam relações entre esses parâmetros, possibilitando que se formulem elementos com um menor número deles.

PILKEY et al. (1995): apresentam matrizes de rigidez, de massa e geométricas para um elemento de viga de Timoshenko, considerando o acoplamento da flexão nas direções principais y e $\mathrm{z}$, por meio dos fatores de forma $\alpha_{\mathrm{yz}}$ e $\alpha_{\mathrm{zy}}$. As equações diferenciais da flexão envolvendo os fatores acima são dadas no capítulo 4.

Na tabela 1, extraída de TESSLER \& DONG (1981), está um sumário dos elementos acima referidos, com suas principais características.

Com relação ao cálculo do fator de forma, podem-se citar os seguintes trabalhos:

COWPER (1966): utilizando as expressões das tensões de cisalhamento e do empenamento $\chi$ no plano da seção transversal obtidas da teoria da elasticidade, o autor deduz uma expressão para o cálculo do fator de forma $\mathrm{k}_{\mathrm{s}}$ (razão entre a distorção média na seção e a distorção no centróide). Esses conceitos serão melhor detalhados no capítulo 4. O autor apresenta no final do artigo uma tabela com as seções transversais mais comumente utilizadas e seus respectivos fatores de forma, dados em função de suas dimensões. As únicas seções transversais abertas de paredes delgadas apresentadas são os perfis I e T. 
Tabela 1 - Sumário de elementos de Timoshenko apresentados

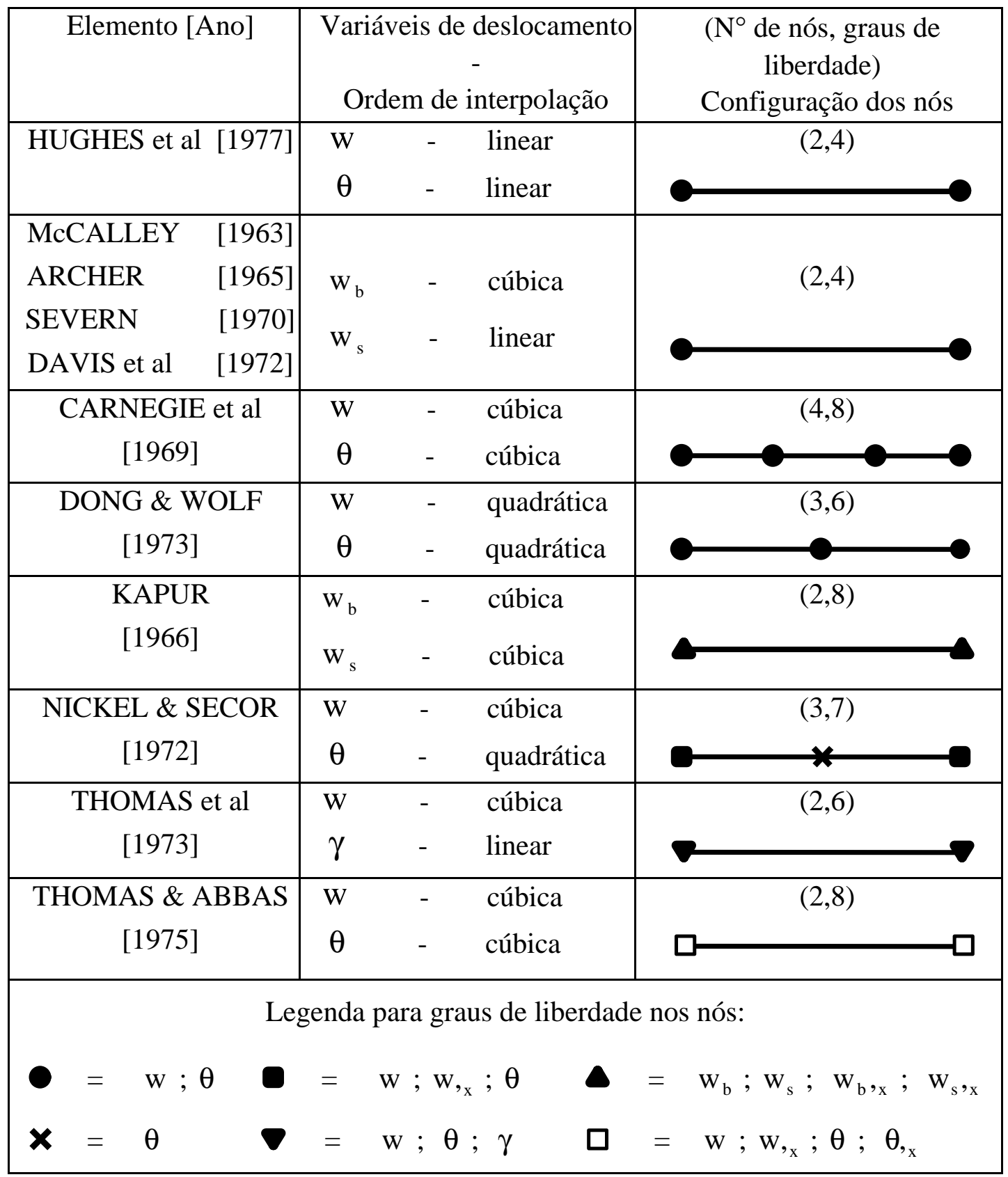

HERRMANN \& MASON (1968): realizam uma dedução semelhante a de Cowper, a qual também resulta em fatores de forma dados em função da integração da função de empenamento $\chi$ na área da seção. As expressões de $\chi$, entretanto, estão disponíveis apenas para determinadas seções e o seu cálculo para seções irregulares é muito complexo. Para contornar essa dificuldade, os autores apresentam uma formulação em elementos finitos triangulares utilizados na discretização da seção transversal em que os parâmetros nodais são os valores de $\chi$. A partir da 
solução obtida, ou seja, dispondo-se dos valores discretos de $\chi$, podem-se calcular os fatores de forma e as tensões de cisalhamento na seção.

SURANA (1979): utiliza o mesmo equacionamento de Herrmann \& Mason e formula elementos isoparamétricos de linha para cálculo da função $\chi$. Estes elementos, resultantes de uma mudança de variáveis feita em elementos bidimensionais, são mais apropriados para discretizar seções de paredes delgadas. A formulação desenvolvida por este autor será apresentada no capítulo 4, no item 4.3.2.

PILKEY et al. (1994): apresentam a dedução da matriz de rigidez e do vetor de forças nodais de um elemento de barra que segue a teoria de Timoshenko pela equação diferencial de equilíbrio, ou seja, não utilizam a formulação de elementos finitos, que se baseia no uso de polinômio interpolador e na minimização da energia potencial total. No que se refere aos fatores de forma, demonstram que ao contrário do que haviam considerado autores anteriores, como HERRMANN \& MASON (1968) e KOSMATKA (1993), os fatores $\alpha_{y z}$ e $\alpha_{z y}$ não se anulam para qualquer seção quando os carregamentos se dão segundo os eixos principais de inércia, mas apenas para as seções com pelo menos um eixo de simetria. Para as seções assimétricas, esses fatores são em geral não-nulos e portanto as flexões segundo as duas direções principais serão acopladas.

Além disso, apresentam duas formulações para cálculo dos fatores de forma de uma seção transversal: a primeira baseia-se num enfoque geométrico, que consiste em estabelecer condições de contorno em termos de deslocamentos e rotações, extraindo relações que permitem obter os fatores de forma; a segunda baseia-se num enfoque energético, em que se igualam a energia de deformação da situação real (tensão cisalhante variando na seção transversal) com a energia de deformação da situação idealizada (tensão cisalhante uniforme na seção e fatores de forma para compatibilizar o trabalho de deformação).

A teoria de Timoshenko apresenta, entretanto, uma deficiência primária, que é a suposição de tensões de cisalhamento uniformes na seção transversal, a qual não permite que se possa atender à condição de contorno de tensões cisalhantes nulas nas extremidades da altura das seções transversais de um elemento fletido. 
Para contornar isso, LEVINSON (1981) introduz a teoria de alta-ordem, adotando para o deslocamento longitudinal de um ponto genérico da seção transversal da viga uma função que varia de forma cúbica com a altura da mesma.

Com isso, é possível impor a condição de contorno de tensões nulas nos extremos da seção. Sua formulação seria posteriormente considerada variacionalmente inconsistente e corrigida por BICKFORD (1982). Em seu trabalho, este autor apresenta soluções analíticas para casos de geometria e carregamentos bastante simples.

A formulação em elementos finitos de barra da teoria de alta-ordem foi apresentada por REDDY \& HEYLIGER (1988), tendo PETROLITO (1995) desenvolvido uma formulação alternativa, que utiliza funções hiperbólicas na aproximação do campo de deslocamentos no interior do elemento.

Como pode ser verificado em TESSLER (1991), PETROLITO (1995) e PLAIS (1998), a teoria de alta-ordem mostra-se mais acurada do que as de EulerBernoulli e de Timoshenko, sendo a que apresenta resultados que mais se aproximam dos obtidos com o uso da teoria da elasticidade.

\section{1 - Dissertações e teses realizadas no Departamento de Estruturas da EESC - USP}

YAGUI (1971): analisa núcleos resistentes utilizando o processo de substituir cada trecho de parede entre lajes por um pilar de rigidez equivalente. $\mathrm{Na}$ formulação da matriz de rigidez do pilar, leva-se em conta a deformação pelo esforço cortante atuante, devido ao carregamento horizontal. Esta abordagem foi utilizada posteriormente por vários autores.

BARBOSA (1978): realiza a análise estrutural de núcleos resistentes contraventados por lintéis, tanto pela técnica do meio contínuo quanto pelo tratamento discreto.

$\mathrm{Na}$ análise pela técnica do meio contínuo, admite-se a flexão dos painéisparede sem considerar, nas equações diferenciais regentes do problema, a deformação pelo esforço cortante. 
No tratamento discreto, utiliza o elemento desenvolvido por TARANATH (1968) que, como visto anteriormente, apresenta sete coordenadas por nó e baseia-se na teoria de Vlassov. No que se refere à submatriz de flexão, a deformação devida ao esforço cortante é desprezada.

COSTA (1982): este trabalho analisa núcleos estruturais contraventados por lintéis sobre fundações flexíveis. São utilizados dois processos: o primeiro utiliza técnicas do meio contínuo, porém analisando apenas o comportamento da estrutura à torção. No segundo, adota-se o modelo empregado por Yagui, havendo consideração da deformação por esforço cortante. Adota-se como fator de forma das seções dos pilares que modelam as paredes o valor dado por COWPER (1966) para seções retangulares $\left(\mathrm{k}_{\mathrm{s}}=\frac{1+\mathrm{v}}{1,2+1,1 \cdot \mathrm{v}}\right)$.

RIBEIRO (1987): seu trabalho tem como principal objetivo a análise da associação tridimensional de pórticos com núcleos resistentes em estruturas de edifícios altos. Utiliza como instrumento as técnicas matriciais e o processo dos deslocamentos.

O modelo adotado para o núcleo é o de barra de sete deslocamentos por nó. No que diz respeito à submatriz de flexão, leva-se em conta a deformação pelo esforço cortante. O programa desenvolvido por esse autor permite a análise de quatro tipos de seção transversal (todas com espessura da parede constante): $\mathrm{U}, \mathrm{H}, \mathrm{C}$ e duplo T. Os seus fatores de forma são calculados a partir de fórmulas dadas em função das dimensões das seções.

SILVA (1989): analisa o comportamento de estruturas tridimensionais de edifícios altos em teoria de primeira ordem e de segunda ordem (efeito $\mathrm{P}-\delta$ ), compostas por pórticos e núcleos resistentes. Estes últimos são analisados como associações de paredes planas, com interações discretizadas no nível das lajes. $\mathrm{Na}$ formulação de suas matrizes de rigidez, leva-se em conta a deformação por esforço cortante do elemento de pilar que é utilizado para representar a rigidez à flexão da parede.

BECKER (1989): desenvolve um programa que analisa a associação tridimensional de pórticos, núcleos e pilares individuais contraventados entre si pelas lajes e vigas. Denominam-se pilares individuais àqueles que apresentam flexão em 
torno de dois eixos principais, além da rotação em torno do eixo longitudinal. Esses pilares podem ser tanto aqueles isolados quanto os situados na intersecção de dois pórticos planos. O programa emprega o método dos deslocamentos e as técnicas de análise matricial e realiza o cálculo em teoria de primeira ordem.

O modelo para os núcleos resistentes é o de barras com sete deslocamentos por nó.

MORI (1992): analisa a interação tridimensional entre pórticos planos, pilares individuais, núcleos estruturais e vigas por meio de um programa que utiliza o processo dos deslocamentos e realiza os cálculos em teorias de primeira e segunda ordens. Os núcleos estruturais podem apresentar seções transversais assimétricas e serem ou não enrijecidos por lintéis.

Nos dois últimos trabalhos, não é considerada, na submatriz de rigidez à flexão do núcleo, a distorção da seção transversal pelo esforço cortante.

SERRA (1994): analisa núcleos resistentes pelo método do pilar de rigidez equivalente à parede. $\mathrm{O}$ efeito da deformação por esforço cortante nas paredes é considerado. É feita a análise em teoria de primeira e de segunda ordens.

MATIAS JR. (1997): analisa a interação tridimensional entre núcleos e as estruturas usuais de contraventamento, como treliças, pórticos e pilares individuais, em primeira e segunda ordens. O programa desenvolvido por este autor permite considerar a flexibilidade das fundações, a influência de trechos rígidos de pilares e as excentricidades entre os eixos longitudinais dos elementos verticais incidentes em um mesmo ponto nodal. Não é considerada, na submatriz de rigidez à flexão do núcleo, a deformação pelo esforço cortante.

PEREIRA (1997): o autor realiza um estudo dos diferentes métodos adotados na análise de edifícios, assim como dos principais modelos adotados para os núcleos resistentes. A seguir, apresenta exemplos numéricos, onde são comparados os resultados obtidos com os modelos de Yagui e de Taranath, além de dois outros, freqüientemente utilizados nos escritórios de cálculo:

- o processo chamado simplificado, que consiste na substituição dos tramos de núcleo entre duas lajes por elementos simples de barra de rigidez equivalente, com seis graus de liberdade por nó, posicionados no centro de torção do núcleo. A rigidez ao empenamento é, portanto, desprezada. 
- o processo dito prático, que consiste em modelar as paredes de um núcleo resistente independentemente, sem considerar as forças de cisalhamento entre elas. 


\section{3 - TEORIAS DE FLEXÃO DE BARRAS}

Sejam os deslocamentos nas direções $\mathrm{x}, \mathrm{y}$ e $\mathrm{z}$ denominados $\mathrm{u}, \mathrm{v}$ e w, respectivamente. A formulação apresentada a seguir irá considerar a flexão apenas em torno do eixo y .

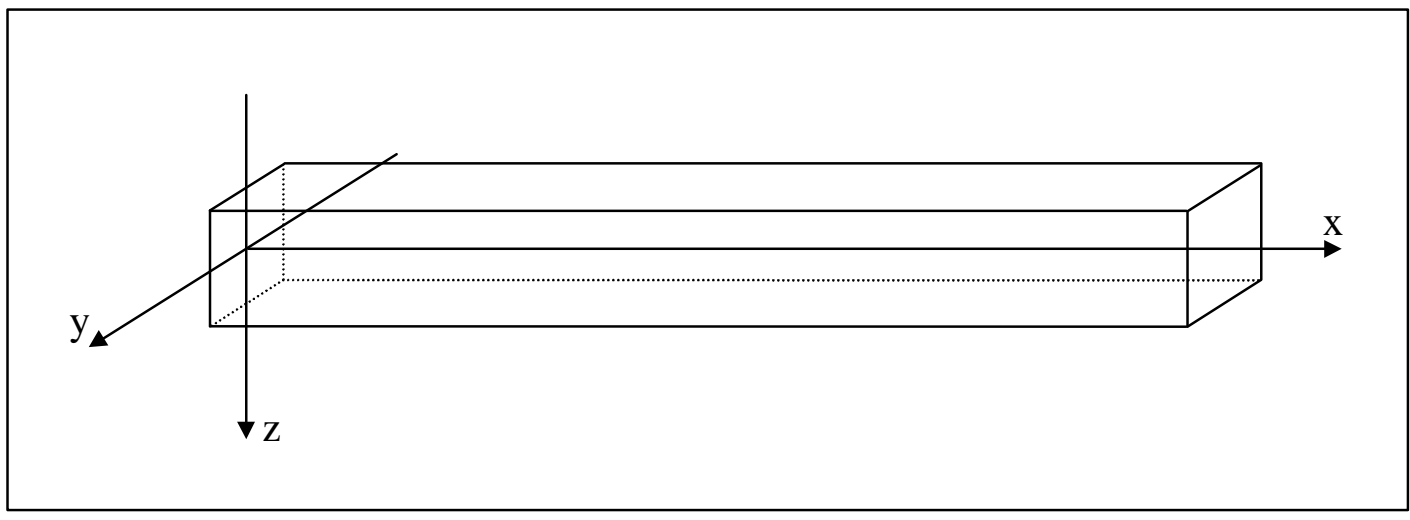

Figura 1 - Sistema de coordenadas (formulação das teorias de

Euler-Bernoulli e de Timoshenko)

Conforme visto no capítulo anterior, são três as teorias de flexão de barras, que são expostas a seguir.

\section{1 - Teoria de Euler-Bernoulli}

Despreza-se a deformação por esforço cortante (distorção) no plano xz . Portanto, seções planas normais ao eixo baricêntrico permanecem planas e normais ao eixo após a flexão, como se vê na figura 2.

Sabe-se que a relação entre o momento numa dada seção transversal e a sua curvatura $\kappa(\kappa=1 / \rho)$, sendo $\rho$ o raio de curvatura do eixo da barra, é dada por:

$$
\mathrm{M}_{\mathrm{y}}=-\mathrm{E} \cdot \mathrm{I}_{\mathrm{y}} \cdot \kappa
$$

E, da geometria analítica: 


$$
\kappa=\frac{\partial \theta / \partial \mathrm{x}}{\left(1+\theta^{2}\right)^{\frac{3}{2}}}
$$

em que $\theta$ é a rotação da seção após a flexão. Para $\theta$ pequeno comparado à unidade a equação (2) se resume a $\kappa=\partial \theta / \partial x$.

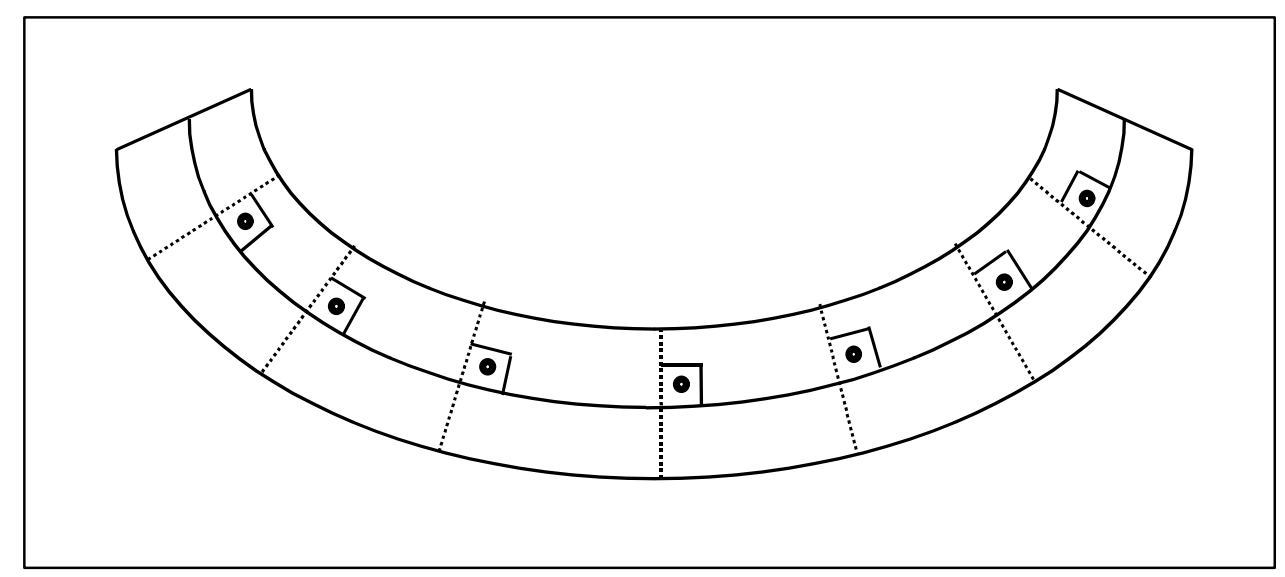

Figura 2 - Flexão pela teoria de Euler-Bernoulli

Além disso, o deslocamento longitudinal de um ponto qualquer da seção transversal é dado por:

$$
\mathrm{u}(\mathrm{x}, \mathrm{z})=\mathrm{u}_{0}(\mathrm{x})-\mathrm{z} \cdot \theta(\mathrm{x})
$$

sendo $\mathrm{u}_{0}(\mathrm{x})$ o deslocamento do centróide na direção do eixo $\mathrm{x}$.

A partir da expressão da distorção no plano xz :

$$
\gamma_{\mathrm{xz}}=\frac{\partial \mathrm{u}}{\partial \mathrm{z}}+\frac{\partial \mathrm{w}}{\partial \mathrm{x}}
$$

escreve-se, sendo, por hipótese, $\gamma_{\mathrm{xz}}=0$ :

$$
\frac{\partial u}{\partial z}+\frac{\partial w}{\partial x}=0 \Rightarrow \frac{\partial w}{\partial x}=\theta
$$

E, portanto, aplicando a eq. (5) na eq. (2), obtém-se:

$$
\kappa=\partial^{2} w / \partial x^{2}
$$

Assim, as equações diferenciais regentes da flexão pela teoria de EulerBernoulli são expressas unicamente em função do deslocamento transversal w :

$$
M_{y}=-E \cdot I_{y} \cdot \frac{\partial^{2} w}{\partial x^{2}}
$$




$$
V_{z}=-E \cdot I_{y} \cdot \frac{\partial^{3} w}{\partial x^{3}}
$$

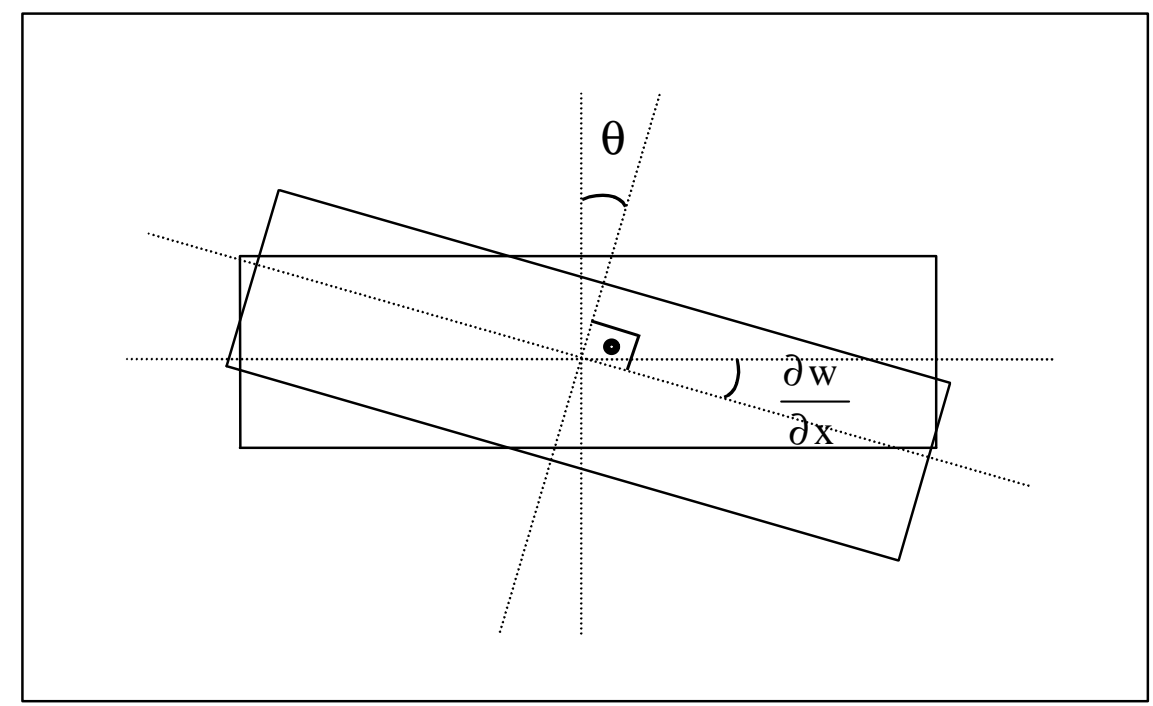

Figura 3 - Rotação da seção transversal (teoria de Euler-Bernoulli)

O elemento de viga mais simples utilizado é o hermitiano com deslocamento transversal regido por polinômio cúbico, com dois nós e dois parâmetros por nó $(\mathrm{w}$ e d w/dx ).

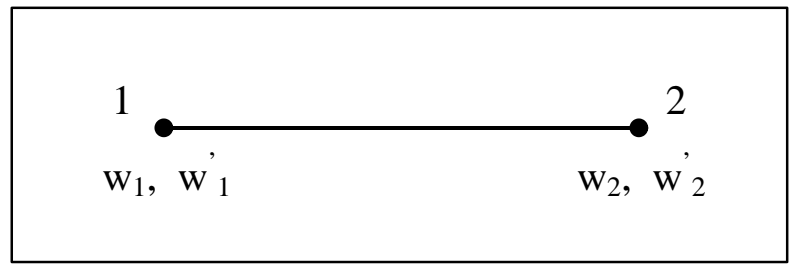

Figura 4 - Parâmetros nodais (teoria de Euler-Bernoulli)

Este elemento apresenta esforço cortante constante ao longo do comprimento da barra e, portanto, a solução apresentada é a exata quando se aplicam forças apenas nos nós.

\section{2 - Teoria de Timoshenko}

Esta teoria leva em conta a deformação pelo esforço cortante. Assume-se que seções planas e normais ao eixo baricêntrico permaneçam planas após a flexão, mas 
não necessariamente normais ao eixo deformado. A variação de rotação corresponde à distorção da seção transversal no plano xz .

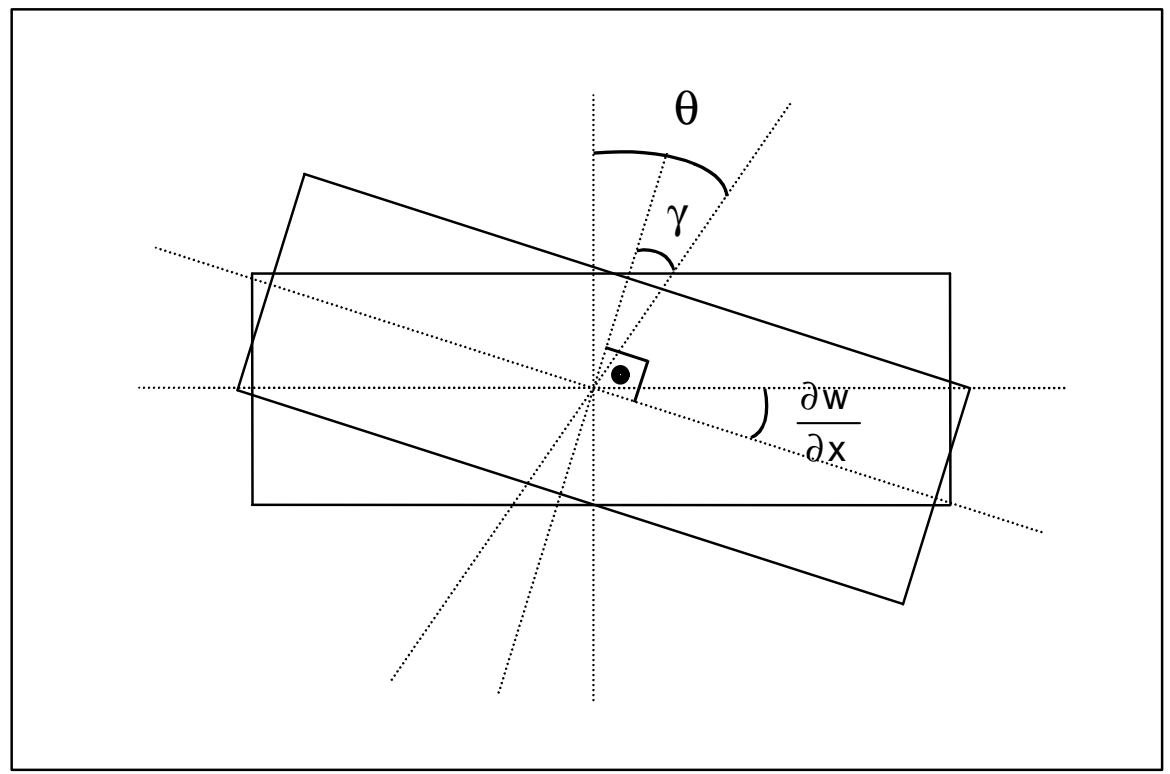

Figura 5 - Rotação da seção transversal (teoria de Timoshenko)

A expressão do deslocamento longitudinal de um ponto da seção transversal é também dada pela eq. (3).

A partir da eq. (4), calcula-se a distorção no plano xz:

$$
\gamma_{\mathrm{xz}}=\frac{\partial \mathrm{u}}{\partial \mathrm{z}}+\frac{\partial \mathrm{w}}{\partial \mathrm{x}} \Rightarrow \gamma_{\mathrm{xz}}=\frac{\partial \mathrm{w}}{\partial \mathrm{x}}-\theta
$$

A suposição de que as seções planas permaneçam planas somente seria válida se todos os pontos da seção transversal tivessem a mesma distorção e, portanto, a mesma tensão de cisalhamento. Sabe-se, entretanto, que a tensão de cisalhamento é variável com relação à altura da viga (com variação quadrática se a largura da seção for constante) e, portanto, a distorção é variável na seção transversal.

Dessa forma, a distorção no centróide da seção transversal $\left(\gamma_{\mathrm{cg}}\right)$ não corresponderá à distorção média da seção $\left(\gamma_{\mathrm{m}}\right)$, dada pela expressão:

$$
\gamma_{\mathrm{m}}=\frac{\mathrm{V}}{\mathrm{G} \cdot \mathrm{A}}
$$

Para efetuar a correção, utiliza-se o parâmetro $\mathrm{k}_{\mathrm{s}}$ chamado, em inglês shear form ou shear stiffness factor, dado por: 


$$
\mathrm{k}_{\mathrm{s}}=\frac{\gamma_{\mathrm{m}}}{\gamma_{\mathrm{cg}}}
$$

E, portanto, das eqs. (10) e (11), chega-se a:

$$
\gamma_{\mathrm{cg}}=\frac{\mathrm{V}}{\mathrm{k}_{\mathrm{s}} \cdot \mathrm{G} \cdot \mathrm{A}}
$$

Define-se também o parâmetro $\alpha\left(\alpha=1 / \mathrm{k}_{\mathrm{s}}\right)$ designado fator de forma, ou, em inglês, shear correction factor, de modo que a expressão anterior se torna:

$$
\gamma_{\mathrm{cg}}=\frac{\alpha \cdot \mathrm{V}}{\mathrm{G} \cdot \mathrm{A}}
$$

As equações diferenciais que regem a flexão nesta teoria são dadas tanto em função do deslocamento transversal do centróide da seção transversal ( w ) quanto da rotação da seção $(\theta)$ :

$$
\begin{aligned}
& M_{y}=-E \cdot I_{y} \cdot \frac{\partial \theta}{\partial x} \\
& E \text {, das eqs. (9) e (12): } \\
& V_{z}=k_{s} \cdot G \cdot A \cdot\left(\frac{\partial w}{\partial x}-\theta\right)
\end{aligned}
$$

A formulação de um número elevado de elementos de viga de Timoshenko (conforme mostrado na tabela 1 do capítulo 2) é viabilizada pela possibilidade de utilizar diferentes aproximações na descrição de w e $\theta$ no interior do elemento, assim como de impor relações entre os parâmetros que definem o deslocamento e a rotação, devido ao fato de existir relação entre momento fletor e esforço cortante $\left(\mathrm{V}=\frac{\mathrm{dM}}{\mathrm{dx}}\right)$.

\section{3 - Teoria de alta-ordem}

Apresentam-se a seguir os passos básicos da dedução das equações diferenciais que regem a flexão de acordo com esta teoria, tendo como base a 
dedução apresentada por PLAIS (1998). O sistema de coordenadas adotado está indicado na figura 6 e esta dedução é válida apenas para seções retangulares.

A suposição básica é admitir um campo de deslocamentos em que o deslocamento longitudinal $\mathrm{u}(\mathrm{x}, \mathrm{z})$ seja uma expansão cúbica da coordenada da altura, como se vê abaixo:

$$
\mathrm{u}(\mathrm{x}, \mathrm{z})=\mathrm{z} \cdot \theta(\mathrm{x})+\mathrm{z}^{2} \cdot \phi(\mathrm{x})+\mathrm{z}^{3} \cdot \psi(\mathrm{x})
$$

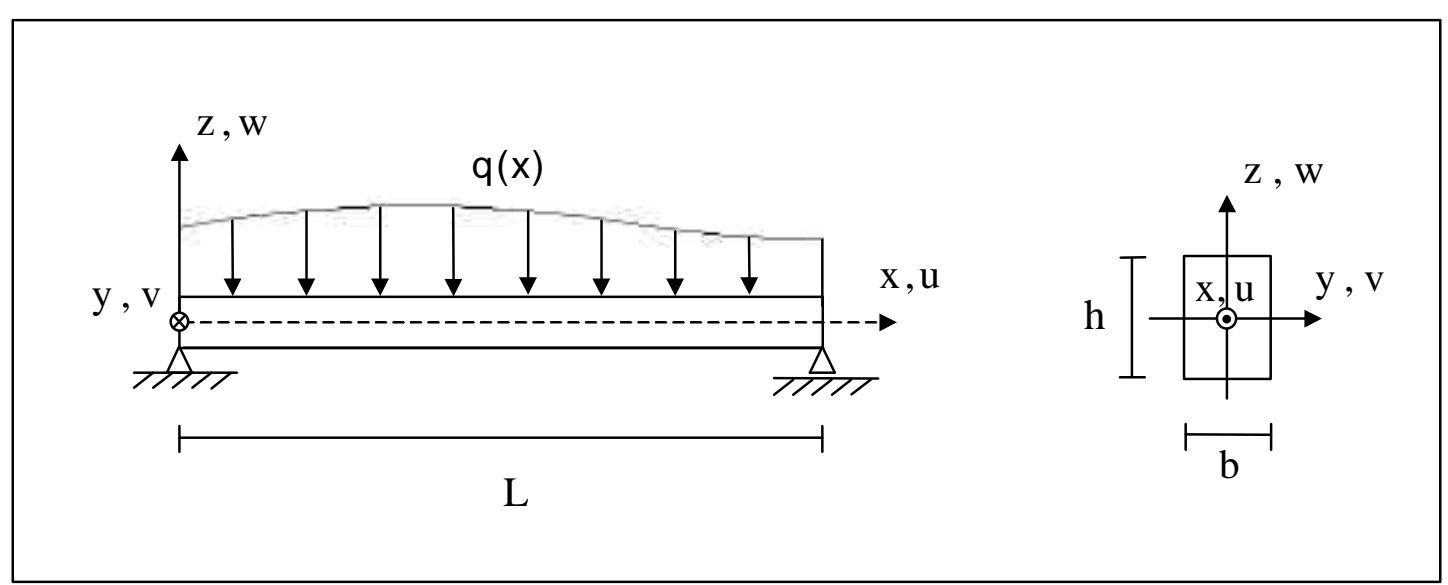

Figura 6 - Sistema de coordenadas (formulação da teoria de alta-ordem)

A função $\theta(\mathrm{x})$ corresponde à rotação da seção transversal enquanto as funções $\phi(x)$ e $\psi(x)$ são incógnitas que serão encontradas pela imposição da condição de contorno de tensão de cisalhamento $\left(\tau_{\mathrm{xz}}\right)$ nula nas extremidades da altura da viga $(\mathrm{z}=-\mathrm{h} / 2$ e $\mathrm{z}=+\mathrm{h} / 2)$. A expressão do deslocamento longitudinal resulta em:

$$
u(x, z)=z \cdot\left\{\theta(x)-\frac{4}{3} \cdot\left(\frac{z}{h}\right)^{2} \cdot\left[\theta(x)+\frac{d w(x)}{d x}\right]\right\}
$$

As deformações normal e transversal associadas a esse campo de deslocamentos são:

$$
\begin{aligned}
& \varepsilon_{x}=z \cdot\left\{\frac{d \theta(x)}{d x}-\frac{4}{3} \cdot\left(\frac{z}{h}\right)^{2} \cdot\left[\frac{d \theta(x)}{d x}+\frac{d^{2} w(x)}{d x^{2}}\right]\right\} \\
& \gamma_{x z}=\left[1-4 \cdot\left(\frac{z}{h}\right)^{2}\right] \cdot\left[\theta(x)+\frac{d w(x)}{d x}\right]
\end{aligned}
$$


A energia potencial total da viga ( $\pi$ ) é dada pela relação:

$$
\pi=\mathrm{U}+\mathrm{V}
$$

na qual a energia de deformação ( U ) e a energia potencial das forças externas (V) são dadas por:

$$
\begin{aligned}
& \mathrm{U}=\frac{1}{2} \cdot \int_{\mathrm{V}} \sigma_{\mathrm{ij}} \cdot \varepsilon_{\mathrm{ij}} \mathrm{dV} \\
& \mathrm{V}=-\int_{0}^{\mathrm{L}} \mathrm{q} \cdot \mathrm{wdx}
\end{aligned}
$$

Admitindo-se que as únicas tensões significativas sejam $\sigma_{x}$ e $\tau_{x z}$ e que o coeficiente de Poisson ( $v$ ) seja nulo, a eq. (21) pode ser escrita assim:

$$
\mathrm{U}=\frac{1}{2} \cdot \int_{\mathrm{V}}\left(\mathrm{E} \cdot \varepsilon_{\mathrm{x}}^{2}+\mathrm{G} \cdot \gamma_{\mathrm{xz}}^{2}\right) \mathrm{dV}
$$

Substituindo na eq. (23) as expressões de $\varepsilon_{\mathrm{x}}$ e $\gamma_{\mathrm{xz}}$ dadas respectivamente pelas eqs. (18) e (19) e realizando a integração na área da seção transversal, chega-se à seguinte expressão da energia de deformação:

$$
\begin{aligned}
& \mathrm{U}=\frac{1}{2} \cdot \int_{0}^{\mathrm{L}}\left[\mathrm{E} \cdot \mathrm{I}_{2} \cdot\left(\frac{\mathrm{d} \theta}{\mathrm{dx}}\right)^{2}-\frac{8 \cdot \mathrm{E} \cdot \mathrm{I}_{4}}{3 \cdot \mathrm{h}^{2}} \cdot\left(\frac{\mathrm{d} \theta}{\mathrm{dx}}\right)^{2}-\frac{8 \cdot \mathrm{E} \cdot \mathrm{I}_{4}}{3 \cdot \mathrm{h}^{2}} \cdot \frac{\mathrm{d} \theta}{\mathrm{dx}} \cdot \frac{\mathrm{d}^{2} \mathrm{w}}{\mathrm{dx}^{2}}+\right. \\
& +\frac{16 \cdot \mathrm{E} \cdot \mathrm{I}_{6}}{9 \cdot \mathrm{h}^{4}} \cdot\left(\frac{\mathrm{d} \theta}{\mathrm{dx}}\right)^{2}+\frac{32 \cdot \mathrm{E} \cdot \mathrm{I}_{6}}{9 \cdot \mathrm{h}^{4}} \cdot \frac{\mathrm{d} \theta}{\mathrm{dx}} \cdot \frac{\mathrm{d}^{2} \mathrm{~W}}{\mathrm{dx}^{2}}+\frac{16 \cdot \mathrm{E} \cdot \mathrm{I}_{6}}{9 \cdot \mathrm{h}^{4}} \cdot\left(\frac{\mathrm{d}^{2} \mathrm{~W}}{\mathrm{dx}^{2}}\right)^{2}+\mathrm{G} \cdot \mathrm{A} \cdot \theta^{2}+ \\
& +2 \cdot \mathrm{G} \cdot \mathrm{A} \cdot \theta \cdot \frac{\mathrm{dw}}{\mathrm{dx}}+\mathrm{G} \cdot \mathrm{A} \cdot\left(\frac{\mathrm{dw}}{\mathrm{dx}}\right)^{2}-\frac{8 \cdot \mathrm{G} \cdot \mathrm{I}_{2}}{\mathrm{~h}^{2}} \cdot \theta^{2}-\frac{16 \cdot \mathrm{G} \cdot \mathrm{I}_{2}}{\mathrm{~h}^{2}} \cdot \theta \cdot \frac{\mathrm{dw}}{\mathrm{dx}}- \\
& \left.-\frac{8 \cdot \mathrm{G} \cdot \mathrm{I}_{2}}{\mathrm{~h}^{2}} \cdot\left(\frac{\mathrm{dw}}{\mathrm{dx}}\right)^{2}+\frac{16 \cdot \mathrm{G} \cdot \mathrm{I}_{4}}{\mathrm{~h}^{4}} \cdot \theta^{2}+\frac{32 \cdot \mathrm{G} \cdot \mathrm{I}_{4}}{\mathrm{~h}^{4}} \cdot \theta \cdot \frac{\mathrm{dw}}{\mathrm{dx}}+\frac{16 \cdot \mathrm{G} \cdot \mathrm{I}_{4}}{\mathrm{~h}^{4}} \cdot\left(\frac{\mathrm{dw}}{\mathrm{dx}}\right)^{2}\right] \mathrm{dx}
\end{aligned}
$$

em que os momentos de inércia superior $\mathrm{I}_{2}, \mathrm{I}_{4}$ e $\mathrm{I}_{6}$ são dados por:

$$
\begin{aligned}
& I_{2}=\int_{A} z^{2} d A \\
& I_{4}=\int_{A} z^{4} d A \\
& I_{6}=\int_{A} z^{6} d A
\end{aligned}
$$


A expressão da energia potencial total $\pi$ é, portanto, um funcional de segunda ordem cujas variáveis independentes são $\mathrm{x}, \theta, \theta^{\prime}, \mathrm{w}, \mathrm{w}^{\prime}$ e w", ou seja:

$$
\pi=\int_{0}^{\mathrm{L}} \mathrm{F}\left(\mathrm{x}, \theta, \theta^{\prime}, \mathrm{w}, \mathrm{w}^{\prime}, \mathrm{w}^{\prime \prime}\right) \mathrm{dx}
$$

As equações de Euler-Lagrange aplicáveis a este caso são:

$$
\begin{aligned}
& \frac{\partial \mathrm{F}}{\partial \theta}-\frac{\mathrm{d}}{\mathrm{dx}}\left(\frac{\partial \mathrm{F}}{\partial \theta^{\prime}}\right)=0 \\
& \frac{\partial \mathrm{F}}{\partial \mathrm{w}}-\frac{\mathrm{d}}{\mathrm{dx}}\left(\frac{\partial \mathrm{F}}{\partial \mathrm{w}^{\prime}}\right)+\frac{\mathrm{d}^{2}}{\mathrm{dx}^{2}}\left(\frac{\partial \mathrm{F}}{\partial \mathrm{w}^{\prime \prime}}\right)
\end{aligned}
$$

Fazendo-se as derivadas indicadas nas eqs. (27) e (28), chega-se às seguintes equações diferenciais:

$$
\begin{aligned}
& \left(\mathrm{G} \cdot \mathrm{A}-\frac{8}{\mathrm{~h}^{2}} \cdot \mathrm{G} \cdot \mathrm{I}_{2}+\frac{16}{\mathrm{~h}^{4}} \cdot \mathrm{G} \cdot \mathrm{I}_{4}\right) \cdot\left(\theta+\mathrm{w}^{\prime}\right)-\frac{16}{9 \cdot \mathrm{h}^{4}} \cdot \mathrm{E} \cdot \mathrm{I}_{6} \cdot\left(\theta^{\prime}+\mathrm{w}^{\prime \prime}\right)+ \\
& +\frac{8}{3 \cdot \mathrm{h}^{2}} \cdot \mathrm{E} \cdot \mathrm{I}_{4} \cdot\left(\theta^{\prime}+\frac{1}{2} \cdot \mathrm{w}^{\prime \prime}\right)-\mathrm{E} \cdot \mathrm{I}_{2} \cdot \theta^{\prime}=0 \\
& \left(\mathrm{G} \cdot \mathrm{A}-\frac{8}{\mathrm{~h}^{2}} \cdot \mathrm{G} \cdot \mathrm{I}_{2}+\frac{16}{\mathrm{~h}^{4}} \cdot \mathrm{G} \cdot \mathrm{I}_{4}\right) \cdot\left(\theta^{\prime}+\mathrm{w}^{\prime \prime}\right)+\frac{16 \cdot \mathrm{E} \cdot \mathrm{I}_{6}}{9 \cdot \mathrm{h}^{4}} \cdot\left(\theta^{\prime \prime \prime}+\mathrm{w}^{\mathrm{IV}}\right)- \\
& -\frac{4 \cdot \mathrm{E} \cdot \mathrm{I}_{4}}{3 \cdot \mathrm{h}^{2}} \cdot \theta^{\prime \prime \prime}=\mathrm{q}
\end{aligned}
$$

Substituem-se nas eqs. (29) e (30) os seguintes valores de $I_{2}, I_{4}$ e $I_{6}$ :

$$
\begin{aligned}
& I_{2}=I \\
& I_{4}=\frac{3 \cdot h^{2}}{20} \cdot I \\
& I_{6}=\frac{3 \cdot h^{4}}{112} \cdot I
\end{aligned}
$$

sendo o momento de inércia $\mathrm{I}=\mathrm{b} \cdot \mathrm{h}^{3} / 12$.

O resultado são as equações diferenciais da flexão de uma viga de seção retangular,segundo a teoria de alta-ordem, dadas abaixo:

$$
\begin{aligned}
& \frac{8}{15} \cdot G \cdot A \cdot\left(\theta^{\prime}+w^{\prime \prime}\right)-\frac{1}{21} \cdot E \cdot I \cdot w^{i v}+\frac{16}{105} \cdot E \cdot I \cdot \theta^{\prime \prime \prime}+q=0 \\
& \frac{8}{15} \cdot G \cdot A \cdot\left(\theta+w^{\prime}\right)-\frac{68}{105} \cdot E \cdot I \cdot \theta^{\prime \prime}+\frac{16}{105} \cdot w^{\prime \prime \prime}=0
\end{aligned}
$$


Uma vez que as soluções analíticas para estas equações são bastante limitadas, o procedimento usual é utilizar técnicas numéricas, ou seja, utilizar polinômios interpoladores para os deslocamentos, aplicá-los ao funcional de energia potencial total e, através da minimização deste último, obter a matriz de rigidez e o vetor de forças nodais da viga.

Embora tanto a teoria de Timoshenko quanto a de alta-ordem permitam incluir na análise estrutural a distorção no plano da seção transversal do núcleo (tratado como barra), a opção neste trabalho foi pela primeira. A razão principal reside na dificuldade de se adaptar a formulação da teoria de alta-ordem à análise de núcleos de seção arbitrária. Uma vez que a teoria de Timoshenko requer o conhecimento dos fatores de forma da seção do núcleo, é utilizado o método semiinverso para calculá-los. É o que se verá no capítulo 4. 


\section{4 - MÉTODO SEMI-INVERSO NA FLEXÃO DE BARRAS - APLICAÇÃO À TEORIA DE TIMOSHENKO}

Este capítulo está dividido em quatro itens. No primeiro, apresenta-se a distribuição da tensão de cisalhamento na seção transversal de uma barra segundo a suposição simplificadora da teoria de Resistência dos Materiais de que a tensão acima referida seja constante segundo a largura da barra.

No segundo item, é apresentada a formulação da flexão de barras segundo um dos métodos usados na teoria da elasticidade, que é o método semi-inverso. A partir desta formulação, calculam-se os fatores de forma da seção, que é um passo necessário para o emprego da teoria de Timoshenko.

No terceiro item, apresenta-se a formulação em elementos finitos utilizada no cálculo do empenamento da seção transversal, dos fatores de forma e da distribuição de tensões na seção.

O último item apresenta as matrizes de rigidez da teoria de Timoshenko que foram utilizadas neste trabalho.

\section{1 - Tensão de cisalhamento na seção transversal de uma barra - suposição da Resistência dos Materiais}

A dedução é feita a partir do equilíbrio de uma fatia de seção $\mathrm{A}_{0}$ de um elemento de barra, como indicado nas figuras abaixo. Admite-se flexão apenas em torno do eixo $\mathrm{z}$ e que a tensão $\tau_{\mathrm{xy}}$ seja constante ao longo da largura $\mathrm{b}$.

Sendo $\mathrm{F}$ a força axial total nos extremos da fatia, dada por:

$$
\mathrm{F}=\int_{\mathrm{A}_{0}} \sigma_{\mathrm{x}} \mathrm{dA}
$$

em que $\sigma_{\mathrm{x}}$ é a tensão normal na seção da barra. 
Do equilíbrio indicado na figura 8 , obtém-se:

$$
\tau_{\mathrm{xy}}=\frac{1}{\mathrm{~b}} \cdot \frac{\partial \mathrm{F}}{\partial \mathrm{x}}
$$
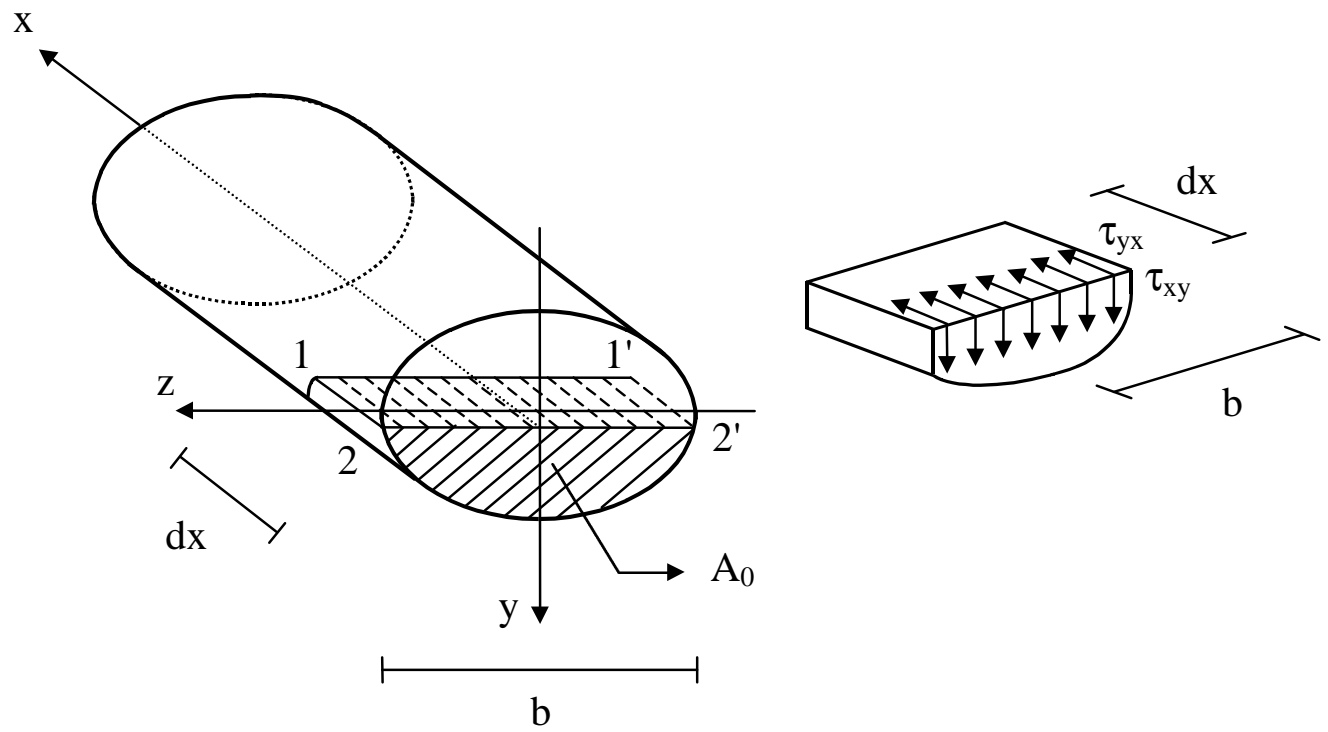

Figura 7 - Corte de uma fatia de viga

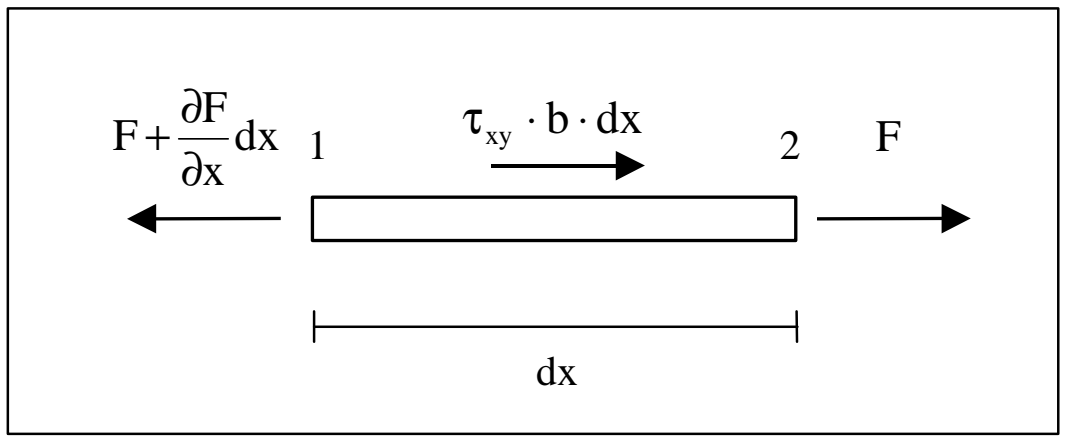

Figura 8 - Equilíbrio da fatia de viga, em vista lateral

E, das eqs. (34) e (35), chega-se a:

$\tau_{\mathrm{xy}}=\frac{1}{\mathrm{~b}} \cdot \int_{\mathrm{A}_{0}} \frac{\partial \sigma_{\mathrm{x}}}{\partial \mathrm{x}} \mathrm{dA}$

Sendo a expressão da tensão normal na seção do elemento:

$\sigma_{\mathrm{x}}=\frac{\mathrm{M}_{\mathrm{z}}}{\mathrm{I}_{\mathrm{z}}} \cdot \mathrm{y}$

Obtém-se a tensão cisalhante, substituindo a eq. (37) na eq. (36): 


$$
\tau_{\mathrm{xy}}=\frac{\mathrm{V}_{\mathrm{y}} \cdot \mathrm{S}_{\mathrm{z}}}{\mathrm{b} \cdot \mathrm{I}_{\mathrm{z}}}
$$

Sendo $\mathrm{S}_{\mathrm{z}}$ o momento estático da área hachurada da seção transversal em relação ao eixo z e $V_{y}$ o esforço cortante na seção.

Essa expressão, todavia, deve ser vista apenas como uma aproximação razoável, já que a suposição de que a tensão de cisalhamento não varie ao longo da largura da barra geralmente não é válida.

Podem ser obtidas melhores soluções para a distribuição da tensão de cisalhamento a partir da teoria da elasticidade. Soluções exatas para essa distribuição só estão disponíveis para determinadas seções transversais e para poucos tipos de carregamento e de condições de contorno, como é o caso da viga engastada com uma força aplicada na extremidade livre.

Entretanto, sabe-se que a distribuição da tensão de cisalhamento na seção é a mesma tanto quando se aplica força concentrada na extremidade como força uniformemente distribuída no comprimento da barra. Assume-se, então, que se possa extrapolar a relação funcional entre esforço cortante e a distribuição das tensões na seção transversal obtida no caso de força na extremidade para outros casos de carregamento (além da força uniformemente distribuída), e também para diferentes condições de contorno.

Um processo de análise de tensões freqüentemente empregado na teoria da elasticidade é o método semi-inverso. Nesta abordagem, fazem-se suposições simplificadoras em relação a alguns componentes de tensão, deformação ou deslocamento, deixando graus de liberdade para satisfazer as condições de equilíbrio e de compatibilidade. Esse método será utilizado no próximo item. 


\section{2 - Método semi-inverso aplicado à flexão de barras - cálculo de fatores de forma e da distribuição das tensões de cisalhamento na seção transversal}

A dedução será feita para a seguinte convenção de forças transversais e momentos fletores positivos, que não é a usualmente empregada em Resistência dos Materiais.

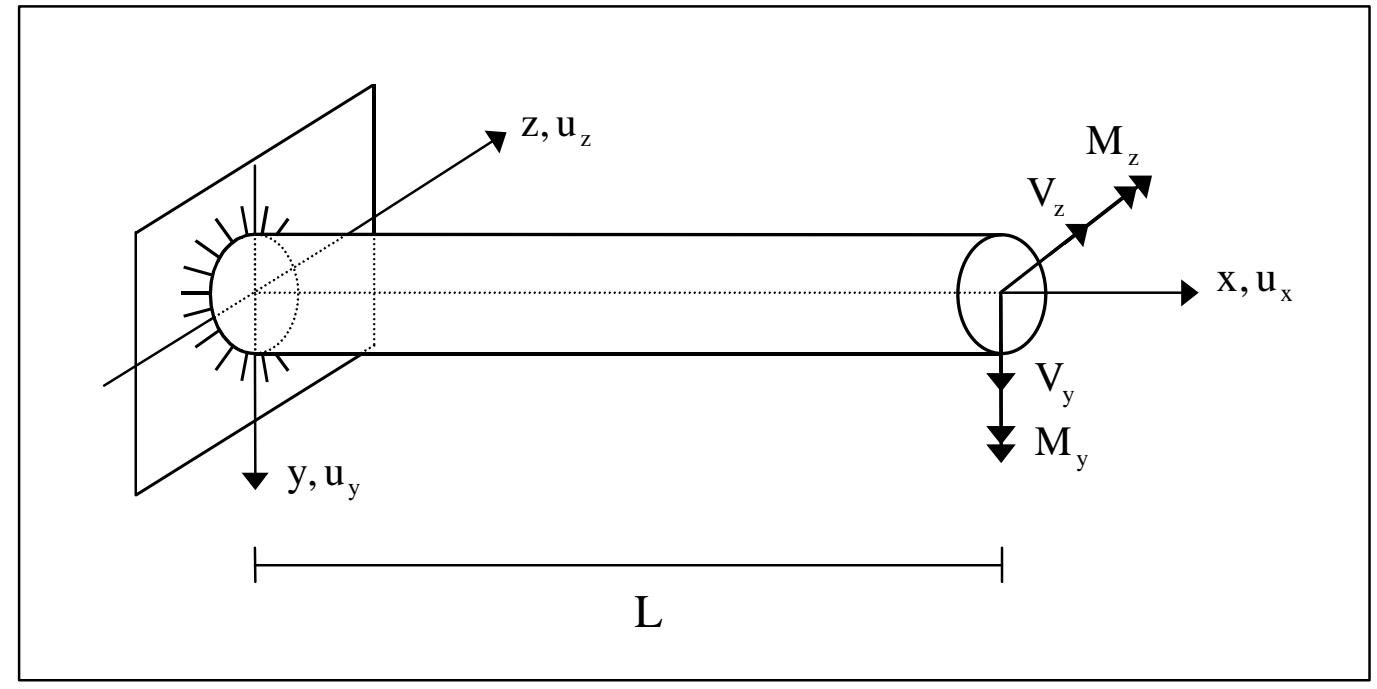

Figura 9 - Convenção de forças transversais e momentos fletores positivos

Admite-se que a seção seja indeformável no seu plano (Hipótese de Wagner) e que portanto as tensões normais $\sigma_{\mathrm{y}}$ e $\sigma_{\mathrm{z}}$ e a tensão de cisalhamento $\tau_{\mathrm{yz}}$ sejam nulas.

Admite-se a extremidade esquerda engastada e aplicam-se cargas concentradas $\mathrm{V}_{\mathrm{y}}$ e $\mathrm{V}_{\mathrm{z}}$ na extremidade livre, que irão produzir os seguintes momentos fletores:

$$
\begin{aligned}
& M_{y}=-V_{z} \cdot(L-x) \\
& M_{z}=V_{y} \cdot(L-x)
\end{aligned}
$$

A tensão normal $\sigma_{\mathrm{x}}$ é dada por:

$$
\sigma_{x}=\frac{M_{y}}{I_{y}} \cdot z-\frac{M_{z}}{I_{z}} \cdot y
$$


Adotando-se os parâmetros:

$$
\begin{aligned}
& \mathrm{k}_{\mathrm{y}}=\frac{\mathrm{V}_{\mathrm{y}}}{\mathrm{E} \cdot \mathrm{I}_{\mathrm{z}}} \\
& \mathrm{k}_{\mathrm{z}}=\frac{\mathrm{V}_{\mathrm{z}}}{\mathrm{E} \cdot \mathrm{I}_{\mathrm{y}}}
\end{aligned}
$$

Pode-se escrever, substituindo as eqs. (39) e (40) na eq. (41) e simplificando através das eq. (42) e (43):

$$
\sigma_{\mathrm{x}}=-\mathrm{E} \cdot(\mathrm{L}-\mathrm{x}) \cdot\left(\mathrm{y} \cdot \mathrm{k}_{\mathrm{y}}+\mathrm{z} \cdot \mathrm{k}_{\mathrm{z}}\right)
$$

Substitui-se a expressão de $\sigma_{x}$ nas relações constitutivas e aplicam-se as relações de compatibilidade:

$$
\begin{aligned}
& \varepsilon_{x}=\frac{\partial u_{x}}{\partial x}=\frac{\sigma_{x}}{E}=-(L-x) \cdot\left(y \cdot k_{y}+z \cdot k_{z}\right) \\
& \varepsilon_{y}=\frac{\partial u_{y}}{\partial y}=\frac{-v \cdot \sigma_{x}}{E}=v \cdot(L-x) \cdot\left(y \cdot k_{y}+z \cdot k_{z}\right) \\
& \varepsilon_{z}=\frac{\partial u_{z}}{\partial z}=\frac{-v \cdot \sigma_{x}}{E}=v \cdot(L-x) \cdot\left(y \cdot k_{y}+z \cdot k_{z}\right)
\end{aligned}
$$

Integrando as eqs. (45), (46) e (47), chega-se aos deslocamentos $u_{x}, u_{y} e$ $\mathrm{u}_{\mathrm{z}}$ :

$$
\begin{aligned}
& u_{x}=-\left(L \cdot x-\frac{1}{2} \cdot x^{2}\right) \cdot\left(y \cdot k_{y}+z \cdot k_{z}\right)+h(y, z) \\
& u_{y}=v \cdot(L-x) \cdot\left(\frac{1}{2} \cdot y^{2} \cdot k_{y}+y \cdot z \cdot k_{z}\right)+g_{1}(x, z) \\
& u_{z}=v \cdot(L-x) \cdot\left(\frac{1}{2} \cdot z^{2} \cdot k_{z}+y \cdot z \cdot k_{y}\right)+g_{2}(x, y)
\end{aligned}
$$

em que $h(y, z), g_{1}(x, z)$ e $g_{2}(x, y)$ são funções de integração.

As deformações $\gamma_{x y}$ e $\gamma_{x z}$ podem então ser expressas por:

$$
\begin{aligned}
\gamma_{x y}=\frac{\partial u_{x}}{\partial y}+\frac{\partial u_{y}}{\partial x}= & -\left(L \cdot x-\frac{1}{2} \cdot x^{2}\right) \cdot k_{y}-v \cdot\left(\frac{1}{2} \cdot y^{2} \cdot k_{y}+y \cdot z \cdot k_{z}\right)+ \\
& +\frac{\partial h}{\partial y}+\frac{\partial g_{1}}{\partial x}
\end{aligned}
$$




$$
\begin{aligned}
\gamma_{x z}=\frac{\partial u_{x}}{\partial z}+\frac{\partial u_{z}}{\partial x}= & -\left(L \cdot x-\frac{1}{2} \cdot x^{2}\right) \cdot k_{z}-v \cdot\left(\frac{1}{2} \cdot z^{2} \cdot k_{z}+y \cdot z \cdot k_{y}\right)+ \\
& +\frac{\partial h}{\partial z}+\frac{\partial g_{2}}{\partial x}
\end{aligned}
$$

Das equações de equilíbrio para um elemento infinitesimal, sem a aplicação de forças de volume:

$$
\begin{aligned}
& \frac{\partial \tau_{x y}}{\partial x}+\frac{\partial \sigma_{y}}{\partial y}+\frac{\partial \tau_{z y}}{\partial z}=0 \Rightarrow \frac{\partial \tau_{x y}}{\partial x}=0, \text { pois } \quad \sigma_{y}=\tau_{z y}=0 \\
& \frac{\partial \tau_{x z}}{\partial x}+\frac{\partial \tau_{y z}}{\partial y}+\frac{\partial \sigma_{z}}{\partial z}=0 \Rightarrow \frac{\partial \tau_{x z}}{\partial x}=0, \text { pois } \quad \sigma_{z}=\tau_{y z}=0
\end{aligned}
$$

Uma vez que:

$$
\begin{aligned}
& \tau_{\mathrm{xy}}=\mathrm{G} \cdot \gamma_{\mathrm{xy}} \\
& \tau_{\mathrm{xz}}=\mathrm{G} \cdot \gamma_{\mathrm{xz}}
\end{aligned}
$$

Chega-se a que:

$$
\begin{aligned}
& \frac{\partial \gamma_{x y}}{\partial x}=0 \\
& \frac{\partial \gamma_{x z}}{\partial x}=0
\end{aligned}
$$

Substituindo nas eqs. (57) e (58) as expressões dadas nas eqs. (51) e (52), obtém-se:

$$
\begin{aligned}
& -(L-x) \cdot k_{y}+\frac{\partial^{2} g_{1}}{\partial x^{2}}=0 \\
& -(L-x) \cdot k_{z}+\frac{\partial^{2} g_{2}}{\partial x^{2}}=0
\end{aligned}
$$

Integrando-se essas relações, obtém-se:

$$
\begin{aligned}
& \mathrm{g}_{1}(\mathrm{x}, \mathrm{z})=\left(\frac{1}{2} \cdot \mathrm{L} \cdot \mathrm{x}^{2}-\frac{1}{6} \cdot \mathrm{x}^{3}\right) \cdot \mathrm{k}_{\mathrm{y}}+\varphi_{1}(\mathrm{z}) \cdot \mathrm{x}+\varphi_{2}(\mathrm{z}) \\
& \mathrm{g}_{2}(\mathrm{x}, \mathrm{y})=\left(\frac{1}{2} \cdot \mathrm{L} \cdot \mathrm{x}^{2}-\frac{1}{6} \cdot \mathrm{x}^{3}\right) \cdot \mathrm{k}_{\mathrm{z}}+\varphi_{3}(\mathrm{y}) \cdot \mathrm{x}+\varphi_{4}(\mathrm{y})
\end{aligned}
$$

Dado que $\tau_{\mathrm{yz}}=0$, segue que $\gamma_{\mathrm{yz}}=0$ e portanto a partir da equação: 


$$
\gamma_{\mathrm{yz}}=\frac{\partial \mathrm{u}_{\mathrm{y}}}{\partial \mathrm{z}}+\frac{\partial \mathrm{u}_{\mathrm{z}}}{\partial \mathrm{y}}
$$

Obtém-se, aplicando na eq. (63) as eqs. (49), (50), (61) e (62) e agrupando os termos de forma conveniente:

$$
\begin{aligned}
& {\left[\frac{\partial \varphi_{2}(\mathrm{z})}{\partial \mathrm{z}}+\mathrm{v} \cdot \mathrm{L} \cdot \mathrm{z} \cdot \mathrm{k}_{\mathrm{y}}\right]+\left[\frac{\partial \varphi_{4}(\mathrm{y})}{\partial \mathrm{y}}+\mathrm{v} \cdot \mathrm{L} \cdot \mathrm{y} \cdot \mathrm{k}_{\mathrm{z}}\right]+\left[\frac{\partial \varphi_{1}(\mathrm{z})}{\partial \mathrm{z}}-\mathrm{v} \cdot \mathrm{z} \cdot \mathrm{k}_{\mathrm{y}}\right] \cdot \mathrm{x}+} \\
& +\left[\frac{\partial \varphi_{3}(\mathrm{y})}{\partial \mathrm{y}}-\mathrm{v} \cdot \mathrm{y} \cdot \mathrm{k}_{\mathrm{z}}\right] \cdot \mathrm{x}=0
\end{aligned}
$$

Os dois primeiros termos são funções de $\mathrm{z}$ e $\mathrm{y}$, apenas, enquanto os dois últimos são funções de $\mathrm{z}$ e x e de y e x. Como são independentes, cada um dos quatro termos deve anular-se:

$$
\begin{aligned}
& \frac{\partial \varphi_{1}(\mathrm{z})}{\partial \mathrm{z}}=\mathrm{v} \cdot \mathrm{z} \cdot \mathrm{k}_{\mathrm{y}} \Rightarrow \varphi_{1}(\mathrm{z})=\frac{1}{2} \cdot \mathrm{v} \cdot \mathrm{z}^{2} \cdot \mathrm{k}_{\mathrm{y}}+\mathrm{a}_{1} \\
& \frac{\partial \varphi_{2}(\mathrm{z})}{\partial \mathrm{z}}=-\mathrm{v} \cdot \mathrm{L} \cdot \mathrm{z} \cdot \mathrm{k}_{\mathrm{y}} \Rightarrow \varphi_{2}(\mathrm{z})=-\frac{1}{2} \cdot \mathrm{v} \cdot \mathrm{L} \cdot \mathrm{z}^{2} \cdot \mathrm{k}_{\mathrm{y}}+\mathrm{a}_{2} \\
& \frac{\partial \varphi_{3}(\mathrm{y})}{\partial \mathrm{y}}=\mathrm{v} \cdot \mathrm{y} \cdot \mathrm{k}_{\mathrm{z}} \Rightarrow \varphi_{3}(\mathrm{y})=\frac{1}{2} \cdot \mathrm{v} \cdot \mathrm{y}^{2} \cdot \mathrm{k}_{\mathrm{z}}+\mathrm{a}_{3} \\
& \frac{\partial \varphi_{4}(\mathrm{y})}{\partial \mathrm{y}}=-\mathrm{v} \cdot \mathrm{L} \cdot \mathrm{y} \cdot \mathrm{k}_{\mathrm{z}} \Rightarrow \varphi_{4}(\mathrm{y})=-\frac{1}{2} \cdot \mathrm{v} \cdot \mathrm{L} \cdot \mathrm{y}^{2} \cdot \mathrm{k}_{\mathrm{z}}+\mathrm{a}_{4}
\end{aligned}
$$

em que $a_{1}, a_{2}, a_{3}$ e $a_{4}$ são constantes de integração.

Assim, as expressões resultantes de $\mathrm{g}_{1}(\mathrm{x}, \mathrm{z})$ e $\mathrm{g}_{2}(\mathrm{x}, \mathrm{y})$, pela substituição das eqs. (65) e (66) na eq. (61) e das eqs. (67) e (68) na eq. (62) são:

$$
\begin{aligned}
& g_{1}(x, z)=\left(\frac{1}{2} \cdot L \cdot x^{2}-\frac{1}{6} \cdot x^{3}\right) \cdot k_{y}-\frac{1}{2} \cdot v \cdot(L-x) \cdot z^{2} \cdot k_{y}+a_{1} \cdot x+a_{2} \\
& g_{2}(x, y)=\left(\frac{1}{2} \cdot L \cdot x^{2}-\frac{1}{6} \cdot x^{3}\right) \cdot k_{z}-\frac{1}{2} \cdot v \cdot(L-x) \cdot y^{2} \cdot k_{z}+a_{3} \cdot x+a_{4}
\end{aligned}
$$

Substituem-se as eqs. (69) e (70) nas eqs. (49) e (50) encontrando-se os deslocamentos $u_{\mathrm{y}}$ e $u_{\mathrm{z}}$ :

$$
\begin{aligned}
u_{y}= & v \cdot(L-x) \cdot\left[\frac{1}{2} \cdot k_{y} \cdot\left(y^{2}-z^{2}\right)+y \cdot z \cdot k_{z}\right]+\left(\frac{1}{2} \cdot L \cdot x^{2}-\frac{1}{6} \cdot x^{3}\right) \cdot k_{y}+ \\
& +a_{1} \cdot x+a_{2}
\end{aligned}
$$




$$
\begin{aligned}
u_{z}= & v \cdot(L-x) \cdot\left[y \cdot z \cdot k_{y}-\frac{1}{2} \cdot k_{z} \cdot\left(y^{2}-z^{2}\right)\right]+\left(\frac{1}{2} \cdot L \cdot x^{2}-\frac{1}{6} \cdot x^{3}\right) \cdot k_{z}+ \\
& +a_{3} \cdot x+a_{4}
\end{aligned}
$$

As tensões $\tau_{\mathrm{xy}}$ e $\tau_{\mathrm{xz}}$ são agora determinadas, pela substituição das eqs. (69) e (70) nas eqs. (51) e (52), fazendo uso das eqs. (55) e (56):

$$
\begin{aligned}
& \tau_{\mathrm{xy}}=\mathrm{G} \cdot\left\{-\mathrm{v} \cdot\left[\frac{1}{2} \cdot \mathrm{k}_{\mathrm{y}} \cdot\left(\mathrm{y}^{2}-\mathrm{z}^{2}\right)+\mathrm{y} \cdot \mathrm{z} \cdot \mathrm{k}_{\mathrm{z}}\right]+\mathrm{a}_{1}+\frac{\partial \mathrm{h}(\mathrm{y}, \mathrm{z})}{\partial \mathrm{y}}\right\} \\
& \tau_{\mathrm{xz}}=\mathrm{G} \cdot\left\{-\mathrm{v} \cdot\left[\mathrm{y} \cdot \mathrm{z} \cdot \mathrm{k}_{\mathrm{y}}-\frac{1}{2} \cdot \mathrm{k}_{\mathrm{z}} \cdot\left(\mathrm{y}^{2}-\mathrm{z}^{2}\right)\right]+\mathrm{a}_{3}+\frac{\partial \mathrm{h}(\mathrm{y}, \mathrm{z})}{\partial \mathrm{z}}\right\}
\end{aligned}
$$

Define-se então a função de empenamento relacionado ao cisalhamento $\chi$, dada por:

$$
\chi(y, z)=h(y, z)+a_{1} \cdot y+a_{3} \cdot z
$$

Dessa forma, o deslocamento $\mathrm{u}_{\mathrm{x}}$ e as tensões $\tau_{\mathrm{xy}}$ e $\tau_{\mathrm{xz}}$ podem ser expressos, em função de $\chi$, por (substituindo a eq. (75) nas eqs. (48), (73) e (74)):

$$
\begin{aligned}
& \mathrm{u}_{\mathrm{x}}=-\left(\mathrm{L} \cdot \mathrm{x}-\frac{1}{2} \cdot \mathrm{x}^{2}\right) \cdot\left(\mathrm{y} \cdot \mathrm{k}_{\mathrm{y}}+\mathrm{z} \cdot \mathrm{k}_{\mathrm{z}}\right)+\chi(\mathrm{y}, \mathrm{z})-\mathrm{a}_{1} \cdot \mathrm{y}-\mathrm{a}_{3} \cdot \mathrm{z} \\
& \tau_{\mathrm{xy}}=\mathrm{G} \cdot\left\{\frac{\partial \chi}{\partial \mathrm{y}}-\mathrm{v} \cdot\left[\frac{1}{2} \cdot \mathrm{k}_{\mathrm{y}} \cdot\left(\mathrm{y}^{2}-\mathrm{z}^{2}\right)+\mathrm{y} \cdot \mathrm{z} \cdot \mathrm{k}_{\mathrm{z}}\right]\right\} \\
& \tau_{\mathrm{xz}}=\mathrm{G} \cdot\left\{\frac{\partial \chi}{\partial \mathrm{z}}-\mathrm{v} \cdot\left[+\mathrm{y} \cdot \mathrm{z} \cdot \mathrm{k}_{\mathrm{y}}-\frac{1}{2} \cdot \mathrm{k}_{\mathrm{z}} \cdot\left(\mathrm{y}^{2}-\mathrm{z}^{2}\right)\right]\right\}
\end{aligned}
$$

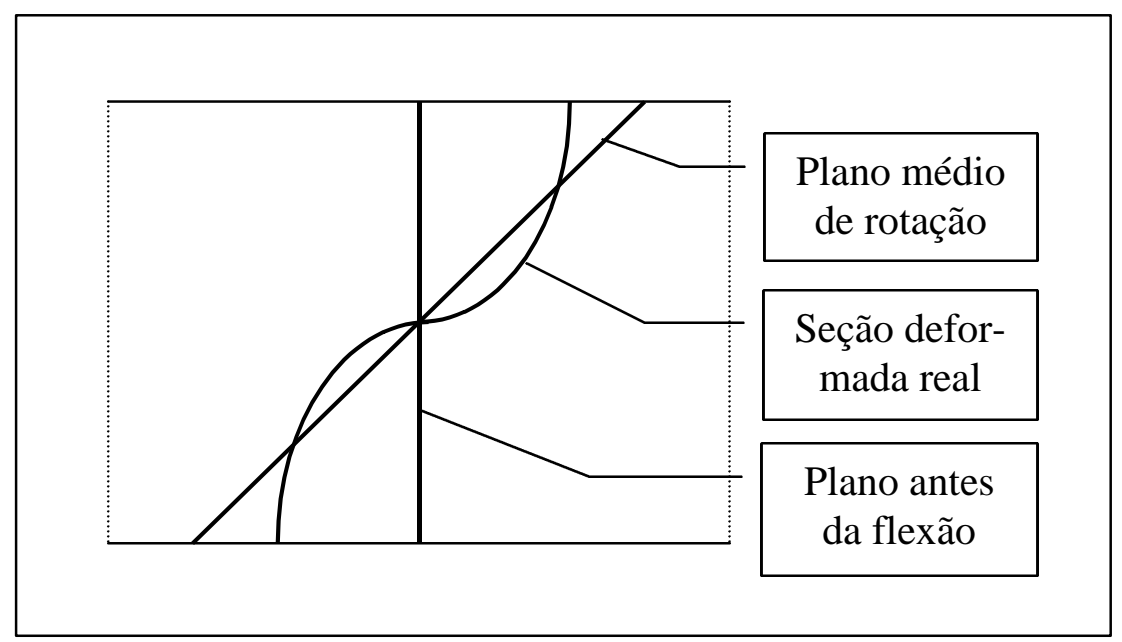

Figura 10 - Seção deformada e seu plano médio de rotação 
Nota-se das expressões de $\tau_{\mathrm{xy}}$ e de $\tau_{\mathrm{xz}}$ que estas são independentes da coordenada $\mathrm{x}$ da barra, das condições de contorno da mesma e do tipo de carregamento aplicado a ela.

Na figura 10, apresenta-se de forma esquemática uma seção deformada de viga em vista lateral, a qual é substituída, na teoria de Timoshenko, por seu plano médio de rotação.

\subsection{1 - Dedução da expressão do fator de forma}

Há agora duas opções para determinar os fatores de forma:

1) Pela imposição de condições de contorno na extremidade engastada da barra, o que permite a determinação das constantes $a_{1}, a_{2}, a_{3}$ e $a_{4}$. Aplicam-se então os deslocamentos $u_{x}, u_{y}$ e $u_{z}$ às equações diferenciais que regem o problema de acordo com a teoria de Timoshenko e obtêm-se os fatores de forma.

2) Pela imposição de que a energia de deformação seja a mesma para a situação descrita pela formulação apresentada anteriormente (distorção variável na seção transversal) e para a situação do modelo de Timoshenko (distorção uniforme e fatores de forma utilizados para correção da diferença entre a distorção no centróide e a distorção média na seção).

A seguir apresentam-se as deduções para cada um dos processos:

1) Pela imposição de condições de contorno em deslocamentos no engaste.

As constantes $\mathrm{a}_{1}, \mathrm{a}_{2}, \mathrm{a}_{3}$ e $\mathrm{a}_{4}$ são encontradas satisfazendo-se a condição de rotações médias e de deslocamentos médios nulos no início da barra.

Em $\mathrm{x}=0$, as integrais $\int_{\mathrm{A}}\left(\mathrm{u}_{\mathrm{x}} \cdot \mathrm{y}\right) \mathrm{dA}$ e $\int_{\mathrm{A}}\left(\mathrm{u}_{\mathrm{x}} \cdot \mathrm{z}\right) \mathrm{dA}$, que são proporcionais aos valores dos ângulos médios de rotação das seções em torno dos eixos z e y, respectivamente, se anulam.

Da primeira integral, substituindo na eq. (76) o valor $\mathrm{x}=0$, calcula-se: 
$\mathrm{a}_{1}=\frac{1}{\mathrm{I}_{\mathrm{z}}} \cdot \int_{\mathrm{A}}(\chi \cdot \mathrm{y}) \mathrm{dA}$

Da segunda integral, substituindo na eq. (76) o valor $\mathrm{x}=0$, calcula-se:

$\mathrm{a}_{3}=\frac{1}{\mathrm{I}_{\mathrm{y}}} \cdot \int_{\mathrm{A}}(\chi \cdot \mathrm{z}) \mathrm{dA}$

Além disso os deslocamentos médios $\overline{\mathrm{u}_{\mathrm{y}}}$ e $\overline{\mathrm{u}_{\mathrm{z}}}$ também se anulam em $\mathrm{x}=0$, sendo:

$$
\begin{aligned}
& \overline{u_{y}}=\frac{1}{A} \cdot \int_{A} u_{y} d A \\
& \overline{u_{z}}=\frac{1}{A} \cdot \int_{A} u_{z} d A
\end{aligned}
$$

Substituindo-se nas integrais acima os valores de $u_{y}$ e $u_{z}$ dados pelas eqs. (59) e (60), sendo $x=0$, obtêm-se as constantes $a_{2}$ e $a_{4}$ :

$$
\begin{aligned}
& a_{2}=-\frac{v \cdot L}{A} \cdot\left[\frac{1}{2} \cdot k_{y} \cdot\left(I_{z}-I_{y}\right)\right] \\
& a_{4}=\frac{v \cdot L}{A} \cdot\left[\frac{1}{2} \cdot k_{z} \cdot\left(I_{z}-I_{y}\right)\right]
\end{aligned}
$$

São agora conhecidas as expressões de $\mathrm{u}_{\mathrm{x}}, \mathrm{u}_{\mathrm{y}}$ e $\mathrm{u}_{\mathrm{z}}$ calculadas pelo método semi-inverso. Na seqüência, essas expressões serão aplicadas às equações diferenciais da flexão (segundo a teoria de Timoshenko), o que permitirá calcular os fatores de forma.

Sendo a força cortante resultante aplicada $V$ e sendo $\alpha_{V}$ o ângulo entre esta força e o eixo y, como mostrado na figura 11, pode-se escrever:

$$
\mathrm{V}=\mathrm{V}_{\mathrm{y}} \cdot \cos \alpha_{\mathrm{V}}+\mathrm{V}_{\mathrm{z}} \cdot \operatorname{sen} \alpha_{\mathrm{V}}
$$

em que $V_{y}$ e $V_{z}$ são as componentes da resultante nas direções y e $\mathrm{z}$.

Sendo $\gamma$ a distorção da seção na direção do esforço cortante resultante $\mathrm{V}$, sua componente na direção do eixo z $\left(\gamma_{\mathrm{xz}}\right)$ é dada por:

$$
\gamma_{\mathrm{xz}}=\operatorname{sen} \alpha_{\mathrm{v}} \cdot \gamma
$$


Substituindo na eq. (86) as expressões de $\gamma$ dada pela eq. (12) e de V dada pela eq. (85), chega-se a:

$$
\gamma_{\mathrm{xz}}=\frac{\mathrm{V}_{\mathrm{y}} \cdot \operatorname{sen} \alpha_{\mathrm{v}} \cdot \cos \alpha_{\mathrm{v}}}{\mathrm{k}_{\mathrm{s}} \cdot \mathrm{G} \cdot \mathrm{A}}+\frac{\mathrm{V}_{\mathrm{z}} \cdot \operatorname{sen}^{2} \alpha_{\mathrm{V}}}{\mathrm{k}_{\mathrm{s}} \cdot \mathrm{G} \cdot \mathrm{A}}
$$

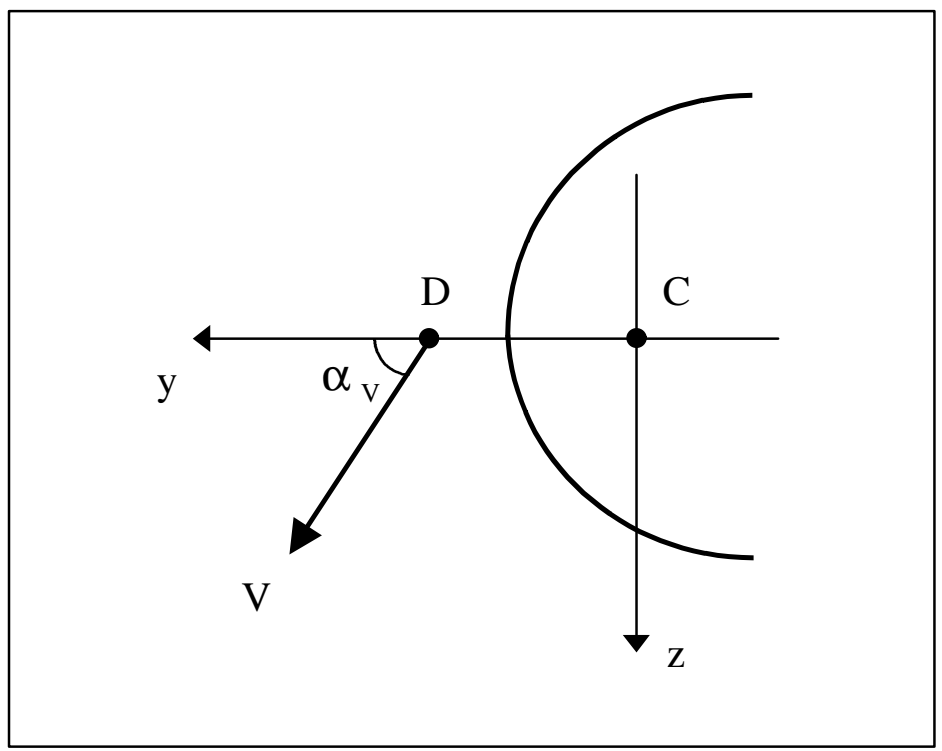

Figura 11 - Força transversal com componentes nas direções y e z

Definindo-se os fatores de cisalhamento:

$$
\begin{aligned}
& \mathrm{k}_{\mathrm{zz}}=\frac{\mathrm{k}_{\mathrm{s}}}{\operatorname{sen}^{2} \alpha_{\mathrm{v}}} \\
& \mathrm{k}_{\mathrm{yz}}=\frac{-\mathrm{k}_{\mathrm{s}}}{\operatorname{sen} \alpha_{\mathrm{v}} \cdot \cos \alpha_{\mathrm{v}}}
\end{aligned}
$$

Pode-se escrever a expressão de $\gamma_{\mathrm{xz}}$ como:

$$
\gamma_{\mathrm{xz}}=\frac{\mathrm{V}_{\mathrm{z}}}{\mathrm{k}_{\mathrm{zz}} \cdot \mathrm{G} \cdot \mathrm{A}}-\frac{\mathrm{V}_{\mathrm{y}}}{\mathrm{k}_{\mathrm{yz}} \cdot \mathrm{G} \cdot \mathrm{A}}
$$

E como se sabe que (na teoria de Timoshenko) as seções planas permanecem planas, pode-se escrever:

$$
\mathrm{u}_{\mathrm{x}}=\mathrm{u}_{\mathrm{cg}}-\mathrm{y} \cdot \theta_{\mathrm{z}}+\mathrm{z} \cdot \theta_{\mathrm{y}}
$$

em que $\theta_{y}$ e $\theta_{z}$ são os ângulos de rotação da seção em torno dos eixos y e z, respectivamente e $u_{c g}$ é o deslocamento na direção do eixo $\mathrm{x}$ do centro de gravidade da seção transversal. 
Sendo $\gamma_{\mathrm{xz}}=\frac{\partial \mathrm{u}_{\mathrm{x}}}{\partial \mathrm{z}}+\frac{\partial \overline{\mathrm{u}_{\mathrm{z}}}}{\partial \mathrm{x}}$, tem-se, utilizando as eqs. (90) e (91):

$$
\frac{d \overline{u_{z}}}{d x}=-\theta_{y}+\alpha_{z z} \cdot \frac{V_{z}}{G \cdot A}-\alpha_{y z} \cdot \frac{V_{y}}{G \cdot A}
$$

com

$$
\begin{aligned}
& \alpha_{\mathrm{zz}}=\frac{1}{\mathrm{k}_{\mathrm{zz}}} \\
& \alpha_{\mathrm{yz}}=\frac{1}{\mathrm{k}_{\mathrm{yz}}}
\end{aligned}
$$

Derivando-se novamente os termos da eq. (92) em relação a x e utilizando a relação entre o momento fletor e a rotação da seção dada pela eq. (14), escreve-se:

$$
\frac{d^{2} \overline{u_{z}}}{d x^{2}}=-\frac{M_{y}}{E \cdot I_{y}}+\frac{\alpha_{z z}}{G \cdot A} \cdot \frac{d V_{z}}{d x}-\frac{\alpha_{y z}}{G \cdot A} \cdot \frac{d V_{y}}{d x}
$$

Utilizando a expressão de $\mathrm{k}_{\mathrm{z}}$ dada pela eq. (43), a expressão anterior resulta em:

$$
\frac{\mathrm{d}^{2} \overline{\mathrm{u}_{\mathrm{z}}}}{\mathrm{dx}^{2}}=(\mathrm{L}-\mathrm{x}) \cdot \mathrm{k}_{\mathrm{z}}+\frac{1}{\mathrm{G} \cdot \mathrm{A}} \cdot\left(\alpha_{\mathrm{zz}} \cdot \frac{\mathrm{dV}_{\mathrm{z}}}{\mathrm{dx}}-\alpha_{\mathrm{yz}} \cdot \frac{\mathrm{dV}_{\mathrm{y}}}{\mathrm{dx}}\right)
$$

De modo similar, pode-se deduzir:

$$
\frac{d^{2} \overline{u_{y}}}{d x^{2}}=(L-x) \cdot k_{y}+\frac{1}{G \cdot A} \cdot\left(\alpha_{y y} \cdot \frac{d V_{y}}{d x}-\alpha_{z y} \cdot \frac{d V z}{d x}\right)
$$

Utilizando-se das expressões de $\overline{u_{y}}$ e $\overline{u_{z}}$ dadas pelas eqs. (81) e (82) e substituindo nas eqs. (96) e (97) chega-se às expressões dos fatores de forma $\alpha_{\mathrm{yy}}$,

$$
\begin{aligned}
\alpha_{\mathrm{yz}}, \alpha_{\mathrm{zy}} \text { e } & \alpha_{\mathrm{zz}}: \\
\alpha_{\mathrm{yy}} & =\frac{1}{\mathrm{I}_{\mathrm{z}}} \cdot\left[\frac{\mathrm{G} \cdot \mathrm{A}}{\mathrm{V}_{\mathrm{y}}} \cdot \int_{\mathrm{A}}(\chi \cdot \mathrm{y}) \mathrm{dA}-\frac{\mathrm{v}}{4 \cdot(1+\mathrm{v})} \cdot\left(\mathrm{I}_{\mathrm{z}}-\mathrm{I}_{\mathrm{y}}\right)\right] \\
\alpha_{\mathrm{yz}} & =\frac{1}{\mathrm{I}_{\mathrm{y}}} \cdot\left[-\frac{\mathrm{G} \cdot \mathrm{A}}{\mathrm{V}_{\mathrm{y}}} \cdot \int_{\mathrm{A}}(\chi \cdot \mathrm{z}) \mathrm{dA}\right] \\
\alpha_{\mathrm{zy}} & =\frac{1}{\mathrm{I}_{\mathrm{z}}} \cdot\left[-\frac{\mathrm{G} \cdot \mathrm{A}}{\mathrm{V}_{\mathrm{z}}} \cdot \int_{\mathrm{A}}(\chi \cdot \mathrm{y}) \mathrm{dA}\right]
\end{aligned}
$$




$$
\alpha_{\mathrm{zz}}=\frac{1}{\mathrm{I}_{\mathrm{y}}} \cdot\left[\frac{\mathrm{G} \cdot \mathrm{A}}{\mathrm{V}_{\mathrm{z}}} \cdot \int_{\mathrm{A}}(\chi \cdot \mathrm{z}) \mathrm{dA}+\frac{\mathrm{V}}{4 \cdot(1+\mathrm{v})} \cdot\left(\mathrm{I}_{\mathrm{z}}-\mathrm{I}_{\mathrm{y}}\right)\right]
$$

As expressões acima relacionadas de $\alpha_{\mathrm{yy}}$ e $\alpha_{\mathrm{yz}}$ foram obtidas considerando carregamento apenas na direção $\mathrm{y}, \operatorname{com} \mathrm{V}_{\mathrm{z}}=0$ e as expressões de $\alpha_{z y}$ e $\alpha_{z z}$ foram obtidas com carregamento apenas na direção $\mathrm{z}, \operatorname{com} \mathrm{V}_{\mathrm{y}}=0$.

2) Pela igualdade de energia de deformação.

A energia de deformação referente ao cisalhamento, considerando distorção uniforme na seção, pode ser escrita como:

$$
\mathrm{U}=\frac{1}{2} \cdot \iint_{\mathrm{L}}\left(\tau_{\mathrm{xy}} \cdot \gamma_{\mathrm{xy}}+\tau_{\mathrm{xz}} \cdot \gamma_{\mathrm{xz}}\right) \mathrm{dAdx}
$$

A expressão de $\gamma_{\mathrm{xz}}$ dada pela eq. (90) e alterada pelas eqs. (93) e (94) é a seguinte:

$$
\gamma_{\mathrm{xz}}=\alpha_{\mathrm{zz}} \cdot \frac{\mathrm{V}_{\mathrm{z}}}{\mathrm{G} \cdot \mathrm{A}}-\alpha_{\mathrm{yz}} \cdot \frac{\mathrm{V}_{\mathrm{y}}}{\mathrm{G} \cdot \mathrm{A}}
$$

A expressão de $\gamma_{\mathrm{xy}}$, análoga à anterior é:

$$
\gamma_{\mathrm{xy}}=\alpha_{\mathrm{yy}} \cdot \frac{\mathrm{V}_{\mathrm{y}}}{\mathrm{G} \cdot \mathrm{A}}-\alpha_{\mathrm{zy}} \cdot \frac{\mathrm{V}_{\mathrm{z}}}{\mathrm{G} \cdot \mathrm{A}}
$$

Substituindo as eqs. (103) e (104) na eq. (102) e utilizando as relações a seguir:

$$
\begin{aligned}
& V_{y}=\int_{A} \tau_{x y} d A \\
& V_{z}=\int_{A} \tau_{x z} d A
\end{aligned}
$$

Obtém-se a seguinte expressão para a energia de deformação:

$$
\mathrm{U}=\frac{1}{2} \cdot \int_{\mathrm{L}}\left(\alpha_{\mathrm{yy}} \cdot \frac{\mathrm{V}_{\mathrm{y}}{ }^{2}}{\mathrm{G} \cdot \mathrm{A}}-\alpha_{\mathrm{yz}} \cdot \frac{\mathrm{V}_{\mathrm{y}} \cdot \mathrm{V}_{\mathrm{z}}}{\mathrm{G} \cdot \mathrm{A}}-\alpha_{\mathrm{zy}} \cdot \frac{\mathrm{V}_{\mathrm{y}} \cdot \mathrm{V}_{\mathrm{z}}}{\mathrm{G} \cdot \mathrm{A}}+\alpha_{\mathrm{zz}} \cdot \frac{\mathrm{V}_{\mathrm{z}}{ }^{2}}{\mathrm{G} \cdot \mathrm{A}}\right) \mathrm{dx}
$$

Para as tensões e distorções na seção variando conforme a teoria da elasticidade, a expressão da energia de deformação é: 


$$
\mathrm{U}=\frac{1}{2 \cdot \mathrm{G}} \cdot \int_{\mathrm{LA}} \int_{\mathrm{A}}\left(\tau_{\mathrm{xy}}{ }^{2}+\tau_{\mathrm{xz}}{ }^{2}\right) \mathrm{dAdx}
$$

Nesta dedução, é conveniente dividir a função de empenamento $\chi$ em duas parcelas, sendo a primeira relacionada à aplicação da força $V_{y}$ e a segunda relacionada à aplicação de $\mathrm{V}_{\mathrm{z}}$ :

$$
\chi=\frac{\mathrm{k}_{\mathrm{y}}}{\mathrm{I}_{\mathrm{y}}} \cdot \chi_{\mathrm{y}}+\frac{\mathrm{k}_{\mathrm{z}}}{\mathrm{I}_{\mathrm{z}}} \cdot \chi_{\mathrm{z}}
$$

Substituindo a eq. (109) nas expressões de $\tau_{x y}$ e $\tau_{x z}$ dadas pelas eqs. (77) e (78), estas passam a ser escritas assim:

$$
\begin{gathered}
\tau_{\mathrm{xy}}=\frac{\left(\mathrm{V}_{\mathrm{y}} \cdot \beta_{\mathrm{yy}}+\mathrm{V}_{\mathrm{z}} \cdot \beta_{\mathrm{zy}}\right)}{2 \cdot(1+\mathrm{v}) \cdot \mathrm{I}_{\mathrm{y}} \cdot \mathrm{I}_{\mathrm{z}}} \\
\tau_{\mathrm{xz}}=\frac{\left(\mathrm{V}_{\mathrm{y}} \cdot \beta_{\mathrm{yz}}+\mathrm{V}_{\mathrm{z}} \cdot \beta_{\mathrm{zz}}\right)}{2 \cdot(1+v) \cdot \mathrm{I}_{\mathrm{y}} \cdot \mathrm{I}_{\mathrm{z}}}
\end{gathered}
$$

Sendo que $\beta_{y y}, \beta_{y z}, \beta_{z y}$ e $\beta_{z z}$ são dados por:

$$
\begin{aligned}
& \beta_{y y}=\frac{\partial \chi_{y}}{\partial y}-v \cdot 0.5 \cdot I_{y} \cdot\left(y^{2}-z^{2}\right) \\
& \beta_{y z}=\frac{\partial \chi_{y}}{\partial z}-v \cdot I_{y} \cdot y \cdot z \\
& \beta_{z y}=\frac{\partial \chi_{z}}{\partial y}-v \cdot I_{z} \cdot y \cdot z \\
& \beta_{z z}=\frac{\partial \chi_{z}}{\partial z}-v \cdot 0.5 \cdot I_{z} \cdot\left(z^{2}-y^{2}\right)
\end{aligned}
$$

E substituindo-se as novas expressões de $\tau_{\mathrm{xy}}$ e $\tau_{\mathrm{xz}}$ dadas pelas eqs. (110) e (111) na eq. (108), obtém-se:

$$
\begin{aligned}
\mathrm{U}= & \frac{1}{8 \cdot \mathrm{G} \cdot(1+\mathrm{v})^{2} \cdot \mathrm{I}_{\mathrm{y}}{ }^{2} \cdot \mathrm{I}_{\mathrm{z}}{ }^{2}} \cdot \iint_{\mathrm{L}}\left[\left(\mathrm{V}_{\mathrm{y}} \cdot \beta_{\mathrm{yy}}+\mathrm{V}_{\mathrm{z}} \cdot \beta_{\mathrm{zy}}\right)^{2}+\right. \\
& \left.+\left(\mathrm{V}_{\mathrm{y}} \cdot \beta_{\mathrm{yz}}+\mathrm{V}_{\mathrm{z}} \cdot \beta_{\mathrm{zz}}\right)^{2}\right] \mathrm{dAdx}
\end{aligned}
$$

Impondo-se a igualdade da energia de deformação dada pelas eqs. (107) e (116), obtêm-se os fatores de forma: 


$$
\begin{aligned}
& \alpha_{\mathrm{yy}}=\frac{\mathrm{A}}{4 \cdot(1+v)^{2} \cdot \mathrm{I}_{\mathrm{y}}{ }^{2} \cdot \mathrm{I}_{\mathrm{z}}^{2}} \cdot \int_{\mathrm{A}}\left(\beta_{\mathrm{yy}}{ }^{2}+\beta_{\mathrm{yz}}{ }^{2}\right) \mathrm{dA} \\
& \alpha_{\mathrm{yz}}=\alpha_{\mathrm{zy}}=-\frac{\mathrm{A}}{4 \cdot(1+v)^{2} \cdot \mathrm{I}_{\mathrm{y}}^{2} \cdot \mathrm{I}_{\mathrm{z}}^{2}} \cdot \int_{\mathrm{A}}\left(\beta_{\mathrm{yy}} \cdot \beta_{\mathrm{zy}}+\beta_{\mathrm{yz}} \cdot \beta_{\mathrm{zz}}\right) \mathrm{dA} \\
& \alpha_{\mathrm{zz}}=\frac{\mathrm{A}}{4 \cdot(1+v)^{2} \cdot \mathrm{I}_{\mathrm{y}}{ }^{2} \cdot \mathrm{I}_{\mathrm{z}}{ }^{2}} \cdot \int_{\mathrm{A}}\left(\beta_{\mathrm{zy}}{ }^{2}+\beta_{\mathrm{zz}}{ }^{2}\right) \mathrm{dA}
\end{aligned}
$$

Os fatores $\beta_{\mathrm{yy}}$ e $\beta_{\mathrm{yz}}$ são calculados com carregamento apenas na direção y e os fatores $\beta_{z y}$ e $\beta_{z z}$, com carregamento apenas na direção $z$.

Embora tenham sido deduzidos para uma situação particular, os coeficientes $\alpha_{y y}, \alpha_{y z}, \alpha_{z y}$ e $\alpha_{z z}$ são propriedades da seção transversal que independem do tipo de carregamento e das condições de contorno, conforme pode ser notado em suas expressões.

O cálculo dos fatores de forma pelo processo geométrico leva a que os fatores $\alpha_{y z}$ e $\alpha_{z y}$ não sejam necessariamente idênticos, como ocorre quando se calcula pelo processo energético. Esse fato faz com que não se possam utilizar os valores obtidos pelo processo geométrico na montagem de matrizes de rigidez que apresentam os termos $\alpha_{y z}$ e $\alpha_{z y}$, pois estas resultariam assimétricas.

Nota-se também que os deslocamentos $u_{x}, u_{y}$ e $u_{z}$, as tensões $\tau_{x y}$ e $\tau_{x z}$ e os fatores de forma $\alpha_{y y}, \alpha_{y z}, \alpha_{z y}$ e $\alpha_{z z}$ são funções de $\chi(y, z)$. A equação diferencial para esta função pode ser obtida pela substituição das expressões das tensões nas equações diferenciais de equilíbrio e impondo condições de contorno (tensão de cisalhamento nula na direção perpendicular à borda). A solução dessas equações diferenciais leva à obtenção da função $\chi$.

Este procedimento torna-se, entretanto, muito trabalhoso quando se trata de seções irregulares. Para o cálculo do empenamento em seções arbitrárias, é útil o emprego de técnicas computacionais.

Dentre os métodos numéricos que podem ser aplicados a este problema, destacam-se: o método dos elementos finitos, o método dos elementos de contorno e o método da integração direta do contorno, entre outros. Neste trabalho, optou-se 
pela resolução pelo método dos elementos finitos, cuja formulação será vista no item seguinte.

\section{3 - Formulação em elementos finitos para cálculo da função de empenamento $\chi$}

Aplica-se o princípio dos trabalhos virtuais (PTV) para uma barra engastada numa extremidade e com carregamento transversal $V_{y}$ e $V_{z}$ na outra:

$$
-\delta \mathrm{W}=\int_{\mathrm{V}}\left(\delta \gamma_{\mathrm{xy}} \cdot \tau_{\mathrm{xy}}+\delta \gamma_{\mathrm{xz}} \cdot \tau_{\mathrm{xz}}\right) \mathrm{dV}-\delta \mathrm{u}_{\mathrm{y} \mid \mathrm{x}=\mathrm{L}} \cdot \mathrm{V}_{\mathrm{y}}-\delta \mathrm{u}_{\mathrm{z} \mid \mathrm{x}=\mathrm{L}} \cdot \mathrm{V}_{\mathrm{z}}
$$

Das expressões de $\tau_{x y}$ e $\tau_{x z}$ dadas nas eqs. (77) e (78), mediante alteração pelas eqs. (55) e (56) obtém-se:

$$
\begin{aligned}
& \delta \gamma_{\mathrm{xy}}=\delta\left\{\frac{\partial \chi}{\partial \mathrm{y}}-\mathrm{v} \cdot\left[\frac{1}{2} \cdot \mathrm{k}_{\mathrm{y}}\left(\mathrm{y}^{2}-\mathrm{z}^{2}\right)+\mathrm{k}_{\mathrm{z}} \cdot \mathrm{y} \cdot \mathrm{z}\right]\right\}=\delta \frac{\partial \chi}{\partial \mathrm{y}}=\frac{\partial(\delta \chi)}{\partial \mathrm{y}} \\
& \delta \gamma_{\mathrm{xz}}=\delta\left\{\frac{\partial \chi}{\partial \mathrm{z}}-\mathrm{v} \cdot\left[\mathrm{k}_{\mathrm{y}} \cdot \mathrm{y} \cdot \mathrm{z}-\frac{1}{2} \cdot \mathrm{k}_{\mathrm{z}}\left(\mathrm{y}^{2}-\mathrm{z}^{2}\right)\right]\right\}=\delta \frac{\partial \chi}{\partial \mathrm{z}}=\frac{\partial(\delta \chi)}{\partial \mathrm{z}}
\end{aligned}
$$

Das expressões de $\mathrm{u}_{\mathrm{y}}$ e $\mathrm{u}_{\mathrm{z}}$, dadas pelas eqs. (71) e (72), substituindo-se os valores de $a_{1}, a_{2}, a_{3}$ e $a_{4}$ dados pelas eqs (79), (83), (80) e (84), para $x=L$ :

$$
\begin{aligned}
& \mathrm{u}_{\mathrm{y} \mid \mathrm{x}=\mathrm{L}}=\frac{1}{3} \cdot \mathrm{L}^{3} \cdot \mathrm{k}_{\mathrm{y}}+\frac{1}{\mathrm{I}_{\mathrm{z}}} \cdot \int_{\mathrm{A}}(\chi \cdot \mathrm{y}) \mathrm{dA}-\frac{\mathrm{v} \cdot \mathrm{L}}{2 \cdot \mathrm{A}} \cdot \mathrm{k}_{\mathrm{y}} \cdot\left(\mathrm{I}_{\mathrm{z}}-\mathrm{I}_{\mathrm{y}}\right) \\
& \mathrm{u}_{\mathrm{z} \mid \mathrm{x}=1}=\frac{1}{3} \cdot \mathrm{L}^{3} \cdot \mathrm{k}_{\mathrm{z}}+\frac{1}{\mathrm{I}_{\mathrm{y}}} \cdot \int_{\mathrm{A}}(\chi \cdot \mathrm{z}) \mathrm{dA}+\frac{\mathrm{v} \cdot \mathrm{L}}{2 \cdot \mathrm{A}} \cdot \mathrm{k}_{\mathrm{z}} \cdot\left(\mathrm{I}_{\mathrm{z}}-\mathrm{I}_{\mathrm{y}}\right)
\end{aligned}
$$

Obtêm-se $\delta u_{y \mid x=L}$ e $\delta u_{z \mid x=L}$ :

$$
\begin{aligned}
& \delta u_{y \mid x=L}=\frac{1}{I_{z}} \cdot \int_{A}(\delta \chi \cdot y) d A \\
& \delta u_{z \mid x=L}=\frac{1}{I_{y}} \cdot \int_{A}(\delta \chi \cdot z) d A
\end{aligned}
$$


Retornando-se à equação do trabalho virtual, e nela substituindo as expressões de $\delta \gamma_{\mathrm{xy}}, \delta \gamma_{\mathrm{xz}}, \delta \mathrm{u}_{\mathrm{y} \mid \mathrm{x}=\mathrm{L}}, \delta \mathrm{u}_{\mathrm{z} \mid \mathrm{x}=\mathrm{L}}, \tau_{\mathrm{xy}}$ e $\tau_{\mathrm{xz}}$, dadas respectivamente pelas eqs. (121), (122), (125), (126), (77) e (78), tem-se, após simplificar:

$$
\begin{aligned}
-\delta W= & G \cdot L \cdot \int_{A}\left[\left(\frac{\partial \chi}{\partial y}-S_{1}\right) \cdot \delta \frac{\partial \chi}{\partial y}+\left(\frac{\partial \chi}{\partial z}-S_{2}\right) \cdot \delta \frac{\partial \chi}{\partial z}\right] d A- \\
& -\frac{V_{y} \cdot L}{I_{z}} \cdot \int_{A}(y \cdot \delta \chi) d A-\frac{V_{z} \cdot L}{I_{y}} \cdot \int_{A}(z \cdot \delta \chi) d A=0
\end{aligned}
$$

em que os parâmetros $S_{1}$ e $S_{2}$ têm por expressão:

$$
\begin{aligned}
& S_{1}=v \cdot\left[\frac{1}{2} \cdot k_{y} \cdot\left(y^{2}-z^{2}\right)+y \cdot z \cdot k_{z}\right] \\
& S_{2}=v \cdot\left[y \cdot z \cdot k_{y}-\frac{1}{2} \cdot k_{z} \cdot\left(y^{2}-z^{2}\right)\right]
\end{aligned}
$$

Utilizando as eqs. (42) e (43), assim como a relação $\mathrm{G}=\mathrm{E} /(2 \cdot(1+\mathrm{v}))$, a equação (127) pode ser assim reescrita:

$$
\begin{gathered}
\int_{\mathrm{A}}\left[\left(\frac{\partial \chi}{\partial \mathrm{y}}-\mathrm{S}_{1}\right) \cdot \delta \frac{\partial \chi}{\partial \mathrm{y}}+\left(\frac{\partial \chi}{\partial \mathrm{z}}-\mathrm{S}_{2}\right) \cdot \delta \frac{\partial \chi}{\partial \mathrm{z}}\right] \mathrm{dA}-2 \cdot(1+v) \cdot\left[\mathrm{k}_{\mathrm{y}} \cdot \int_{\mathrm{A}}(\mathrm{y} \cdot \delta \chi) \mathrm{dA}+\right. \\
\left.\quad+\mathrm{k}_{\mathrm{z}} \cdot \int_{\mathrm{A}}(\mathrm{z} \cdot \delta \chi) \mathrm{dA}\right]=0
\end{gathered}
$$

Fatorando $\delta \chi$ na eq. (130), chega-se a:

$$
\delta \chi \cdot \int_{A}\left[\left({ }_{y} \partial \partial_{y}+{ }_{z} \partial \partial_{z}\right) \chi-S_{1} \cdot y-S_{2} \cdot z-2 \cdot(1+v) \cdot\left(y \cdot k_{y}+z \cdot k_{z}\right)\right] d A=0
$$

O passo seguinte é a subdivisão da seção transversal em elementos, no interior dos quais a função $\chi$ será dada por uma interpolação dos valores nodais.

$$
\chi=\sum_{\mathrm{j}=1}^{\mathrm{n}} \mathrm{N}_{\mathrm{j}} \cdot \chi_{\mathrm{j}}
$$

em que $\quad \chi_{\mathrm{j}}$ é o valor da função $\chi$ no nó $\mathrm{j}$.

$\mathrm{n}$ é o número de nós do elemento.

$\mathrm{N}_{\mathrm{j}}$ são as funções de forma adotadas na interpolação. 
Reescrevendo-se o princípio dos trabalhos virtuais em termos da formulação em elementos finitos, tem-se:

$$
[\mathrm{K}] \cdot\{\chi\}-\{\mathrm{P}\}=0
$$

Sendo $[\mathrm{K}]$ a matriz de rigidez do elemento, que terá como dimensão $\mathrm{n},\{\chi\}$ o vetor de deslocamentos e $\{\mathrm{P}\}$ o vetor de forças nodais.

Os elementos da matriz [K] são dados por:

$$
\mathrm{k}_{\mathrm{ij}}=\int_{\mathrm{A}}\left(\frac{\partial \mathrm{N}_{\mathrm{i}}}{\partial \mathrm{y}} \cdot \frac{\partial \mathrm{N}_{\mathrm{j}}}{\partial \mathrm{y}}+\frac{\partial \mathrm{N}_{\mathrm{i}}}{\partial \mathrm{z}} \cdot \frac{\partial \mathrm{N}_{\mathrm{j}}}{\partial \mathrm{z}}\right) \mathrm{dA}
$$

E os elementos do vetor $\{\mathrm{P}\}$ são dados por:

$$
\mathrm{p}_{\mathrm{i}}=\int_{\mathrm{A}}\left[\mathrm{S}_{1} \cdot \frac{\partial \mathrm{N}_{\mathrm{i}}}{\partial \mathrm{y}}+\mathrm{S}_{2} \cdot \frac{\partial \mathrm{N}_{\mathrm{i}}}{\partial \mathrm{z}}+2 \cdot(1+\mathrm{v}) \cdot \mathrm{N}_{\mathrm{i}} \cdot\left(\mathrm{y} \cdot \mathrm{k}_{\mathrm{y}}+\mathrm{z} \cdot \mathrm{k}_{\mathrm{z}}\right)\right] \mathrm{dA}
$$

Em seções cheias, é usual a sua subdivisão em elementos triangulares ou retangulares, com diferentes graus de polinômio aproximador. Os elementos triangulares levam a vantagem de permitirem que se aproximem contornos irregulares ou mesmo curvos com um erro bem menor do que o dos quadrangulares.

São também amplamente utilizados os elementos finitos chamados isoparamétricos. O princípio fundamental da formulação desses elementos é o fato das funções interpoladoras utilizadas na aproximação dos parâmetros de deslocamento (no caso apenas o empenamento $\chi$ ) serem as mesmas utilizadas para representar a geometria dos elementos, ou seja, para interpolar as coordenadas no interior do elemento em função das coordenadas dos nós.

Com isso, podem-se gerar elementos de forma irregular ("elementos escravos") a partir de um mapeamento das coordenadas de um elemento de formato regular ("elemento mestre"). A modelagem de seções de contorno irregular ou curvo torna-se assim bem mais fácil.

No item seguinte, apresenta-se a formulação de elementos quadrangulares isoparamétricos, com quatro nós por elemento. No item 4.3.2, apresenta-se a formulação de elementos isoparamétricos de linha, que foram utilizados neste trabalho para modelar a seção transversal dos núcleos resistentes. 


\subsection{1 - Elemento quadrangular isoparamétrico}

Seja o elemento quadrangular de quatro nós e coordenadas adimensionais $\xi$ e $\eta$, com $-1 \leq \xi \leq+1$ e $-1 \leq \eta \leq+1$, indicado na figura 12. Este é o chamado elemento mestre.

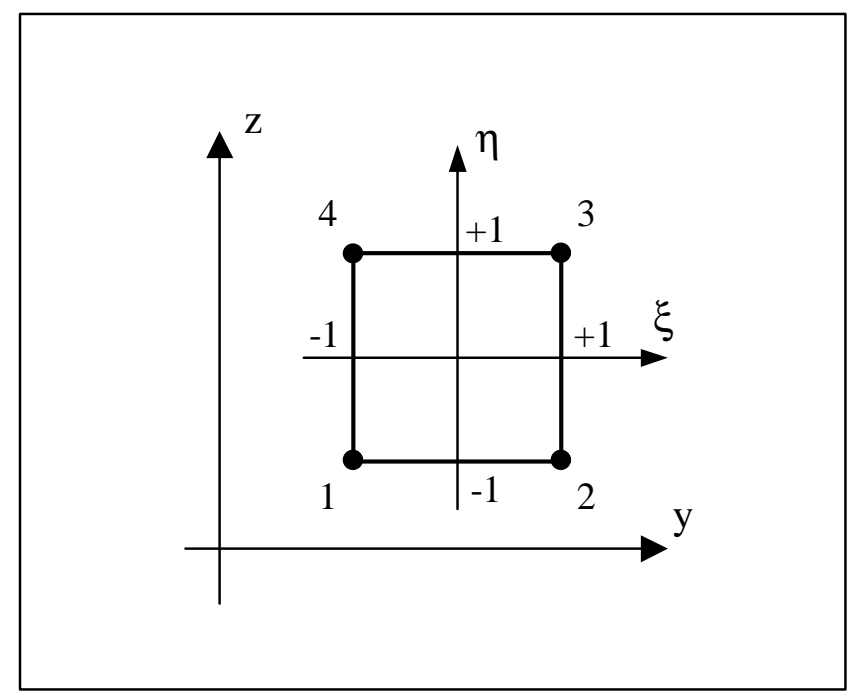

Figura 12 - Elemento quadrangular isoparamétrico

As funções interpoladoras tanto do parâmetro de deslocamento $(\chi)$ como das coordenadas y e z são as seguintes:

$$
\begin{aligned}
& \mathrm{N}_{1}=\frac{1}{4} \cdot(1-\xi) \cdot(1-\eta) \\
& \mathrm{N}_{2}=\frac{1}{4} \cdot(1+\xi) \cdot(1-\eta) \\
& \mathrm{N}_{3}=\frac{1}{4} \cdot(1+\xi) \cdot(1+\eta) \\
& \mathrm{N}_{4}=\frac{1}{4} \cdot(1-\xi) \cdot(1+\eta)
\end{aligned}
$$

O parâmetro $\chi$ no interior do elemento tem sua expressão dada pela eq. (132), aplicando-se a eq. (136).

Utilizando-se a eq. (136) na interpolação das coordenadas y e z, tem-se a descrição das coordenadas no interior do elemento em função das coordenadas dos nós $1\left(\mathrm{y}_{1}, \mathrm{z}_{1}\right), 2\left(\mathrm{y}_{2}, \mathrm{z}_{2}\right), 3\left(\mathrm{y}_{3}, \mathrm{z}_{3}\right)$ e $4\left(\mathrm{y}_{4}, \mathrm{z}_{4}\right)$. 


$$
\begin{aligned}
& \mathrm{y}=\mathrm{N}_{1} \cdot \mathrm{y}_{1}+\mathrm{N}_{2} \cdot \mathrm{y}_{2}+\mathrm{N}_{3} \cdot \mathrm{y}_{3}+\mathrm{N}_{4} \cdot \mathrm{y}_{4} \\
& \mathrm{z}=\mathrm{N}_{1} \cdot \mathrm{z}_{1}+\mathrm{N}_{2} \cdot \mathrm{z}_{2}+\mathrm{N}_{3} \cdot \mathrm{z}_{3}+\mathrm{N}_{4} \cdot \mathrm{z}_{4}
\end{aligned}
$$

Podem-se então utilizar elementos quadrangulares com as mais variadas formas, sendo recomendado apenas que não se utilizem ângulos internos inferiores a $30^{\circ}$ ou superiores a $150^{\circ}$. A figura 13 representa um "elemento escravo".

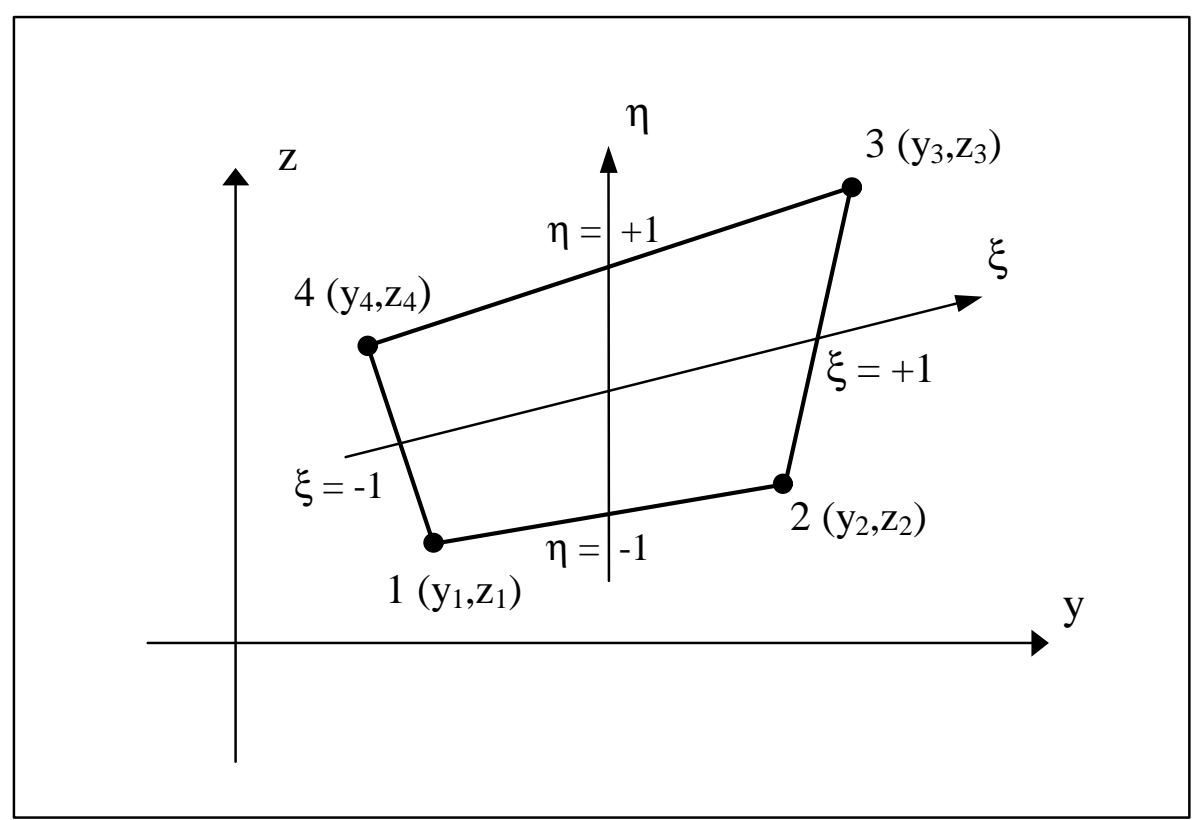

Figura 13 - "Elemento escravo"

Os elementos da matriz de rigidez e do vetor de forças nodais de um elemento isoparamétrico de quatro nós são dados pelas eqs. (139) e (140), com i , j=1,4 :

$$
\begin{aligned}
& \mathrm{k}_{\mathrm{ij}}=\int_{-1-1}^{+1+1}\left[\left(\frac{\partial \mathrm{N}_{\mathrm{i}}}{\partial \mathrm{y}} \cdot \frac{\partial \mathrm{N}_{\mathrm{j}}}{\partial \mathrm{y}}+\frac{\partial \mathrm{N}_{\mathrm{i}}}{\partial \mathrm{z}} \cdot \frac{\partial \mathrm{N}_{\mathrm{j}}}{\partial \mathrm{z}}\right) \cdot \operatorname{det} \mathrm{J}\right] \mathrm{d} \xi \mathrm{d} \eta \\
& \mathrm{p}_{\mathrm{i}}=\int_{-1}^{+1+1} \int_{-1}\left\{\left[\left(\mathrm{~S}_{1} \cdot \frac{\partial \mathrm{N}_{\mathrm{i}}}{\partial \mathrm{y}}+\mathrm{S}_{2} \cdot \frac{\partial \mathrm{N}_{\mathrm{i}}}{\partial \mathrm{z}}\right)+2 \cdot(1+\mathrm{v}) \cdot \mathrm{N}_{\mathrm{i}} \cdot\left(\mathrm{y} \cdot \mathrm{k}_{\mathrm{y}}+\mathrm{z} \cdot \mathrm{k}_{\mathrm{z}}\right)\right] \cdot \operatorname{det} \mathrm{J}\right\} \mathrm{d} \xi \mathrm{d} \eta
\end{aligned}
$$

A matriz $\mathbf{J}$ é o chamado Jacobiano, que é dado por:

$$
J=\left[\begin{array}{ll}
\frac{\partial y}{\partial \xi} & \frac{\partial z}{\partial \xi} \\
\frac{\partial y}{\partial \eta} & \frac{\partial z}{\partial \eta}
\end{array}\right]
$$


A partir desta matriz, obtém-se $\frac{\partial N_{i}}{\partial y}$ e $\frac{\partial N_{i}}{\partial z}$ :

$$
\left\{\begin{array}{c}
\frac{\partial N_{i}}{\partial y} \\
\frac{\partial N_{i}}{\partial z}
\end{array}\right\}=[J]^{-1} \cdot\left\{\begin{array}{c}
\frac{\partial N_{i}}{\partial \xi} \\
\frac{\partial N_{i}}{\partial \eta}
\end{array}\right\}
$$

Uma vez que se tenha a matriz de rigidez e o vetor de forças nodais de cada elemento, é feita a alocação na matriz de rigidez e no vetor de forças nodais de toda a seção transversal, obtendo-se o sistema global. O sistema linear obtido é indeterminado (com apenas um grau de indeterminação) e uma vez que se imponha a condição de contorno (atribuir um valor arbitrário ao parâmetro $\chi$ de qualquer nó da seção), ele torna-se possível e determinado e pode ser resolvido.

Uma vez que se disponha do vetor com os deslocamentos nodais de toda a seção, podem-se calcular os fatores de forma nas direções y e $\mathrm{z}$ pelos dois processos referidos no item anterior:

1) Processo de imposição de condições de contorno nos deslocamentos.

Deve-se resolver o sistema linear duas vezes. $\mathrm{Na}$ primeira aplica-se carregamento $\mathrm{V}_{\mathrm{y}}$ arbitrário, mantendo-se $\mathrm{V}_{\mathrm{z}}=0$. Com o vetor de deslocamentos encontrado, calculam-se:

$$
\begin{aligned}
\alpha_{y y}= & \frac{1}{I_{z}} \cdot\left\{\frac{G \cdot A}{V_{y}} \cdot\left[\sum_{k=1}^{n_{e}} \int_{-1}^{1} \int_{-1}^{1}\left(\sum_{j=1}^{4} N_{j} \cdot \chi_{j}^{k} \cdot \sum_{j=1}^{4} N_{j} \cdot y_{j}^{k}\right) \cdot \operatorname{det} J d \xi d \eta\right]-\right. \\
& \left.-\frac{v}{4 \cdot(1+v)} \cdot\left(I_{z}-I_{y}\right)\right\} \\
\alpha_{y z} & =\frac{1}{I_{y}} \cdot\left\{-\frac{G \cdot A}{V_{y}} \cdot\left[\sum_{k=1}^{n_{e}} \int_{-1}^{1} \int_{-1}^{1}\left(\sum_{j=1}^{4} N_{j} \cdot \chi_{j}^{k} \cdot \sum_{j=1}^{4} N_{j} \cdot z_{j}^{k}\right) \cdot \operatorname{det} J d \xi d \eta\right]\right\}
\end{aligned}
$$

Da segunda vez, aplica-se $\mathrm{V}_{\mathrm{z}}$ arbitrário, mantendo-se $\mathrm{V}_{\mathrm{y}}=0$, e com o vetor de deslocamentos obtidos, calculam-se:

$$
\alpha_{z y}=\frac{1}{I_{z}} \cdot\left\{-\frac{G \cdot A}{V_{z}} \cdot\left[\sum_{k=1}^{n_{e}} \int_{-1}^{1} \int_{-1}^{1}\left(\sum_{j=1}^{4} N_{j} \cdot \chi_{j}^{k} \cdot \sum_{j=1}^{4} N_{j} \cdot y_{j}^{k}\right) \cdot \operatorname{det} J d \xi d \eta\right]\right\}
$$




$$
\begin{aligned}
\alpha_{z z}= & \frac{1}{I_{y}} \cdot\left\{\frac{G \cdot A}{V_{z}} \cdot\left[\sum_{k=1}^{n_{e}} \int_{-1}^{1} \int_{-1}^{1}\left(\sum_{j=1}^{4} N_{j} \cdot \chi_{j}^{k} \cdot \sum_{j=1}^{4} N_{j} \cdot z_{j}^{k}\right) \cdot \operatorname{det} J d \xi d \eta\right]+\right. \\
& \left.+\frac{v}{4 \cdot(1+v)} \cdot\left(I_{z}-I_{y}\right)\right\}
\end{aligned}
$$

Sendo $\mathrm{n}_{\mathrm{e}}$ o número de elementos em que a seção transversal foi dividida.

2) Processo de igualdade da energia de deformação.

O sistema também deve ser resolvido duas vezes. Na primeira com carregamento arbitrário $\mathrm{V}_{\mathrm{y}}$, e fazendo $\mathrm{V}_{\mathrm{z}}=0$, calcula-se o vetor de deslocamentos $\chi$. Multiplicando esse vetor por $\mathrm{I}_{\mathrm{y}} / \mathrm{k}_{\mathrm{y}}$, obtém-se o vetor $\chi_{\mathrm{y}}$. Com estes valores, calculam-se em cada um dos k elementos:

$$
\begin{aligned}
& \beta_{y y}{ }^{k}=\sum_{i=1}^{4} \frac{\partial N_{i}}{\partial y} \cdot \chi_{y_{i}}{ }^{k}-\frac{1}{2} \cdot I_{y} \cdot\left[\left(\sum_{i=1}^{4} N_{i} \cdot y_{i}^{k}\right)^{2}-\left(\sum_{i=1}^{4} N_{i} \cdot z_{i}^{k}\right)^{2}\right] \\
& \beta_{y z}{ }^{k}=\sum_{i=1}^{4} \frac{\partial N_{i}}{\partial z} \cdot \chi_{y_{i}}{ }^{k}-I_{y} \cdot\left(\sum_{i=1}^{4} N_{i} \cdot y_{i}^{k}\right) \cdot\left(\sum_{i=1}^{4} N_{i} \cdot z_{i}^{k}\right)
\end{aligned}
$$

$\mathrm{Na}$ segunda resolução do sistema, aplica-se $\mathrm{V}_{\mathrm{z}}$ arbitrário e mantém-se $\mathrm{V}_{\mathrm{y}}=0$. O vetor de deslocamentos $\chi$ obtido é multiplicado por $\mathrm{I}_{\mathrm{z}} / \mathrm{k}_{\mathrm{z}}$, obtendo-se o vetor $\chi_{\mathrm{z}}$. Calculam-se então em cada elemento $\mathrm{k}$ :

$$
\begin{aligned}
& \beta_{z y}{ }^{k}=\sum_{i=1}^{4} \frac{\partial N_{i}}{\partial y} \cdot \chi_{z i}{ }^{k}-I_{z} \cdot\left(\sum_{i=1}^{4} N_{i} \cdot y_{i}^{k}\right) \cdot\left(\sum_{i=1}^{4} N_{i} \cdot z_{i}^{k}\right) \\
& \beta_{z z}{ }^{k}=\sum_{i=1}^{4} \frac{\partial N_{i}}{\partial z} \cdot \chi_{z i}{ }^{k}-\frac{1}{2} \cdot I_{z} \cdot\left[\left(\sum_{i=1}^{4} N_{i} \cdot y_{i}^{k}\right)^{2}-\left(\sum_{i=1}^{4} N_{i} \cdot z_{i}^{k}\right)^{2}\right]
\end{aligned}
$$

O valor dos fatores de forma é dado então pelo cálculo:

$$
\begin{aligned}
& \alpha_{y y}=\frac{A}{4 \cdot(1+v)^{2} \cdot I_{y} \cdot I_{z}} \cdot\left[\sum_{k=1}^{n_{e}} \int_{-1}^{1} \int_{-1}^{1}\left(\beta_{y y}{ }^{2}+\beta_{y z}{ }^{2}\right) \cdot \operatorname{det} J d \xi d \eta\right] \\
& \alpha_{y z}=\alpha_{z y}=-\frac{A}{4 \cdot(1+v)^{2} \cdot I_{y} \cdot I_{z}} \cdot\left[\sum_{k=1}^{n_{e}} \int_{-1}^{1} \int_{-1}^{1}\left(\beta_{y y}{ }^{k} \cdot \beta_{z y}+\beta_{y z}^{k} \cdot \beta_{z z}{ }^{k}\right) \cdot \operatorname{det} J d \xi d \eta\right] \\
& \alpha_{z z}=\frac{A}{4 \cdot(1+v)^{2} \cdot I_{y} \cdot I_{z}} \cdot\left[\sum_{k=1}^{n_{e}} \int_{-1-1}^{1} \int_{-1}^{1}\left(\beta_{z y}{ }^{k}+\beta_{z z}{ }^{2}\right) \cdot \operatorname{det~Jd} \xi d \eta\right]
\end{aligned}
$$


Já para o cálculo das tensões de cisalhamento, o sistema linear só precisa ser resolvido uma única vez, podendo $\mathrm{V}_{\mathrm{y}}$ e $\mathrm{V}_{\mathrm{z}}$ ser aplicados simultaneamente.

A expressão das tensões de cisalhamento em qualquer ponto no interior de um elemento $\mathrm{k}$ é dada por:

$$
\begin{aligned}
& \tau_{\mathrm{xy}}{ }^{\mathrm{k}}=\mathrm{G} \cdot\left\{\sum_{\mathrm{i}=1}^{4} \frac{\partial \mathrm{N}_{\mathrm{i}}}{\partial \mathrm{y}} \cdot \chi_{\mathrm{i}}{ }^{\mathrm{k}}-\mathrm{v} \cdot\left[\frac{1}{2} \cdot \mathrm{k}_{\mathrm{y}} \cdot\left(\mathrm{y}_{\mathrm{k}}{ }^{2}-\mathrm{z}_{\mathrm{k}}{ }^{2}\right)+\mathrm{y}_{\mathrm{k}} \cdot \mathrm{z}_{\mathrm{k}} \cdot \mathrm{k}_{\mathrm{z}}\right]\right\} \\
& \tau_{\mathrm{xz}}{ }^{\mathrm{k}}=\mathrm{G} \cdot\left\{\sum_{\mathrm{i}=1}^{4} \frac{\partial \mathrm{N}_{\mathrm{i}}}{\partial \mathrm{z}} \cdot \chi_{\mathrm{i}}{ }^{\mathrm{k}}-\mathrm{v} \cdot\left[+\mathrm{y}_{\mathrm{k}} \cdot \mathrm{z}_{\mathrm{k}} \cdot \mathrm{k}_{\mathrm{y}}-\frac{1}{2} \cdot \mathrm{k}_{\mathrm{z}} \cdot\left(\mathrm{y}_{\mathrm{k}}{ }^{2}-\mathrm{z}_{\mathrm{k}}{ }^{2}\right)\right]\right\}
\end{aligned}
$$

Nas eqs. de (143) a (155) é válido considerar:

$\mathrm{n}_{\mathrm{e}}$ o número de elementos em que a seção transversal foi dividida.

$\mathrm{N}_{\mathrm{j}}$ dado pelas eqs. (136).

$\chi_{j}{ }^{k}$ dado pela eq. (132).

$y_{j}^{k}$ e $z_{j}^{k}$ dados pelas eqs.(137) e (138).

\subsection{2 - Elemento isoparamétrico de linha}

No caso de seções transversais de paredes delgadas, como é o caso das seções dos núcleos resistentes, é mais conveniente utilizar elementos finitos de linha na sua discretização. Estes elementos são obtidos pela definição de nós sobre o eixo $\xi$, substituindo os nós pré-existentes. Trata-se, entretanto, apenas de uma mudança dos parâmetros que definem a geometria e o deslocamento, visto que o elemento continua a ter comportamento plano. A razão da maior conveniência da utilização desse elemento reside basicamente no fato de que a entrada de dados é facilitada.

Assim, um elemento de quatro nós torna-se um elemento de linha de dois nós. Um elemento de seis nós (que tem aproximação linear numa das direções e quadrática na outra) irá gerar um elemento de linha de três nós, o qual permitirá uma aproximação quadrática para os deslocamentos e para a geometria. Os dois casos estão indicados na figura 14.

Neste trabalho, optou-se por utilizar o elemento isoparamétrico de linha de dois nós, que apresenta aproximação linear em seu interior tanto para a geometria 
como para o empenamento. Como se pode ver na figura 14 os nós 1 e 4 do elemento quadrangular são substituídos pelo nó 1 do elemento de linha (1e), assim como os nós 2 e 3 são substituídos pelo nó 2 deste elemento (2e ). Estes novos nós estão sobre o eixo $\xi$ do elemento quadrangular.

Uma vez definidos os nós do elemento de linha, passa-se a definir a geometria e o empenamento em seu interior em função de novos parâmetros, conforme será mostrado a seguir.
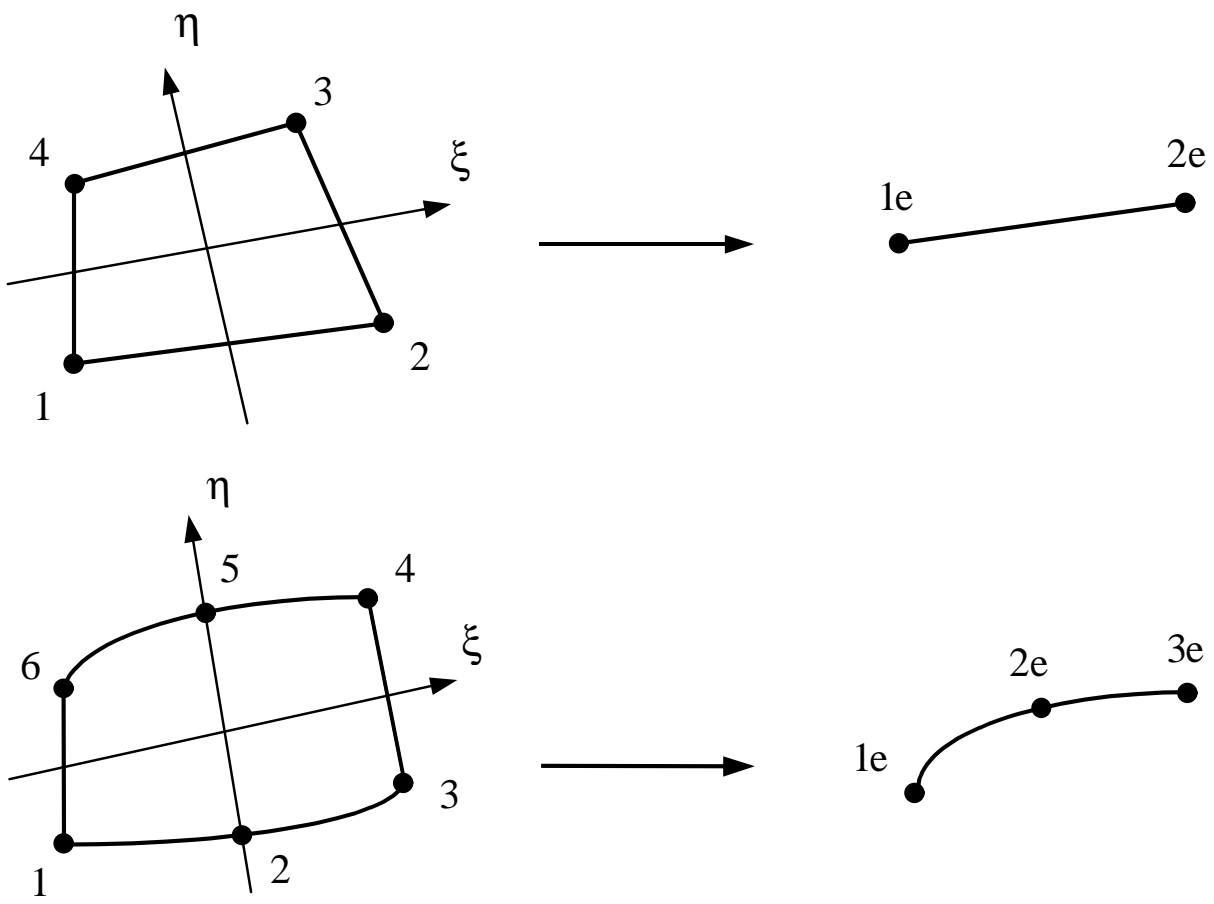

Figura 14 - Transformação de elementos bidimensionais em elementos de linha

Como os nós le e 2 e estão no ponto médio da reta que liga os nós 1 e 4 e 2 e 3, respectivamente, é válido escrever:

$$
\begin{aligned}
& \mathrm{y}_{1 \mathrm{e}}=\frac{\left(\mathrm{y}_{1}+\mathrm{y}_{4}\right)}{2} \\
& \mathrm{z}_{1 \mathrm{e}}=\frac{\left(\mathrm{z}_{1}+\mathrm{z}_{4}\right)}{2} \\
& \mathrm{y}_{2 \mathrm{e}}=\frac{\left(\mathrm{y}_{2}+\mathrm{y}_{3}\right)}{2}
\end{aligned}
$$




$$
\mathrm{z}_{2 \mathrm{e}}=\frac{\left(\mathrm{z}_{2}+\mathrm{z}_{3}\right)}{2}
$$

Sendo $\mathrm{t}_{1}$ a espessura da parede junto ao nó le e $\mathrm{t}_{2}$ a espessura junto ao nó 2e e sendo $\beta$ o ângulo entre o eixo $\xi$ e o eixo y, com sentido positivo de y para $\xi$ no sentido anti-horário, é válido escrever:

$$
\begin{aligned}
& \mathrm{y}_{4}-\mathrm{y}_{1}=-\mathrm{t}_{1} \cdot \operatorname{sen} \beta \\
& \mathrm{z}_{4}-\mathrm{z}_{1}=+\mathrm{t}_{1} \cdot \cos \beta \\
& \mathrm{y}_{3}-\mathrm{y}_{2}=-\mathrm{t}_{2} \cdot \operatorname{sen} \beta \\
& \mathrm{z}_{3}-\mathrm{z}_{2}=+\mathrm{t}_{2} \cdot \cos \beta
\end{aligned}
$$

O ângulo $\beta$ é calculado por:

$$
\beta=\arccos \left(\frac{y_{2 \mathrm{e}}-y_{1 \mathrm{e}}}{\mathrm{l}}\right)
$$

sendo I o comprimento do elemento de linha, dado por:

$$
1=\sqrt{\left(\mathrm{y}_{2 \mathrm{e}}-\mathrm{y}_{1 \mathrm{e}}\right)^{2}+\left(\mathrm{z}_{2 \mathrm{e}}-\mathrm{z}_{1 \mathrm{e}}\right)^{2}}
$$

As relações dadas nas eqs. (156), (157), (158) e (159) podem ser visualizadas na figura 15.

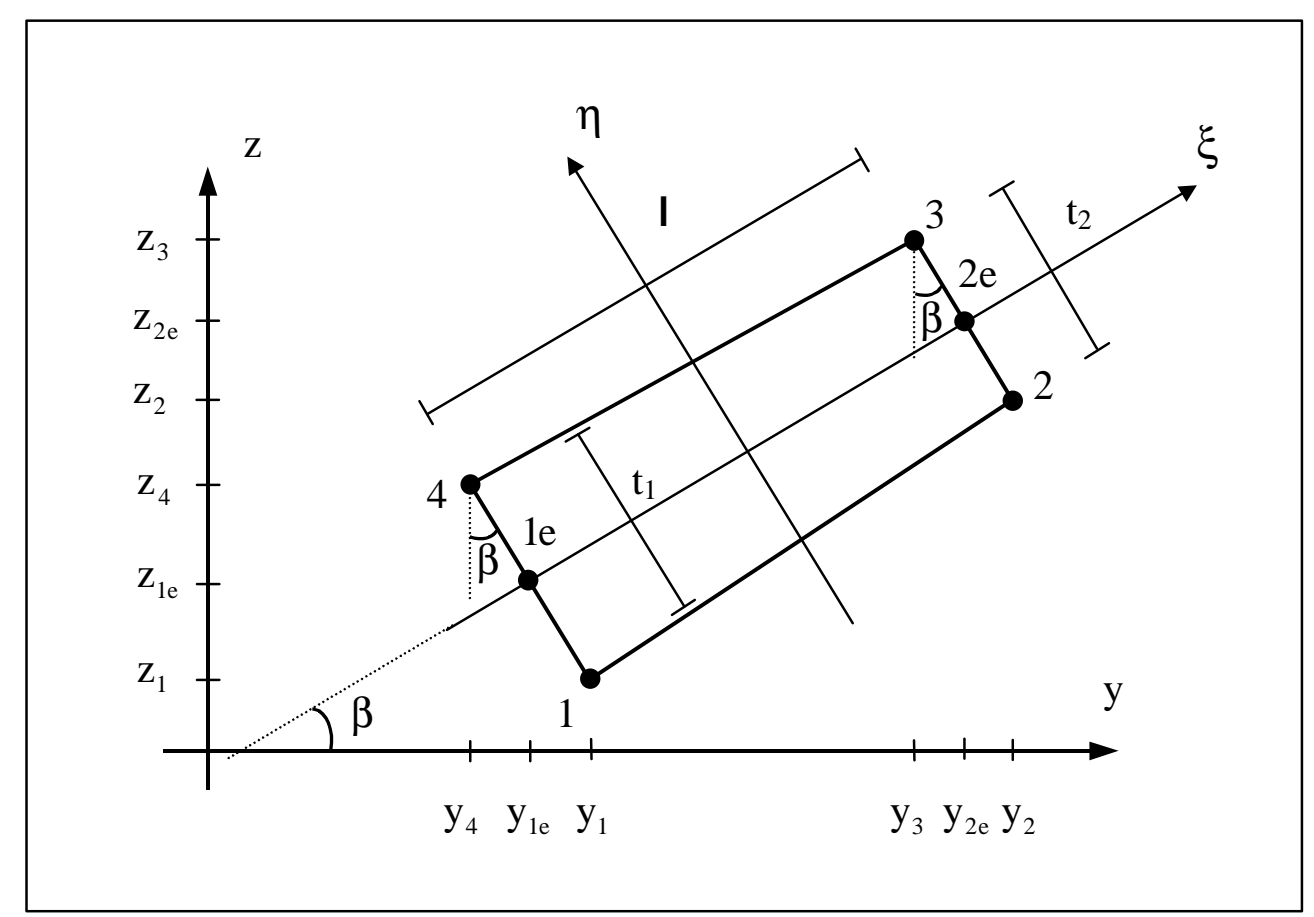

Figura 15 - Definição de parâmetros no elemento de linha 
Partindo das eqs (137) e (138) e utilizando as eqs (156), (157), (158) e (159), podem-se expressar as coordenadas y e $\mathrm{z}$ no interior do elemento de linha como função das coordenadas dos nós 1e e 2e, do ângulo $\beta$ e das espessuras $t_{1}$ e $t_{2}$.

$$
\begin{aligned}
& y=\frac{\left(y_{1 e}+y_{2 e}\right)}{2}+\frac{\left(y_{2 e}-y_{1 e}\right)}{2} \cdot \xi-\operatorname{sen} \beta \cdot \frac{\left(t_{1}+t_{2}\right)}{4} \cdot \eta-\operatorname{sen} \beta \cdot \frac{\left(t_{2}-t_{1}\right)}{4} \cdot \xi \cdot \eta \\
& z=\frac{\left(z_{1 e}+z_{2 e}\right)}{2}+\frac{\left(z_{2 e}-z_{1 e}\right)}{2} \cdot \xi+\cos \beta \cdot \frac{\left(t_{1}+t_{2}\right)}{4} \cdot \eta+\cos \beta \cdot \frac{\left(t_{2}-t_{1}\right)}{4} \cdot \xi \cdot \eta
\end{aligned}
$$

Para que a formulação apresentada anteriormente seja válida, é preciso que as espessuras $t_{1}$ e $t_{2}$ sejam tomadas perpendicularmente ao eixo $\xi$. Como conseqüência, o eixo $\eta$ será também perpendicular ao eixo $\xi$.

Calculando-se o Jacobiano, obtém-se:

$$
J=\left[\begin{array}{cc}
\frac{\left(y_{2 e}-y_{1 e}\right)}{2}-\operatorname{sen} \beta \cdot \frac{\left(t_{2}-t_{1}\right)}{4} \cdot \eta & \frac{\left(z_{2 e}-z_{1 e}\right)}{2}+\cos \beta \cdot \frac{\left(t_{2}-t_{1}\right)}{4} \cdot \eta \\
-\operatorname{sen} \beta \cdot\left[\frac{\left(t_{1}+t_{2}\right)}{4}+\frac{\left(t_{2}-t_{1}\right)}{4} \cdot \xi\right] & \cos \beta \cdot\left[\frac{\left(t_{1}+t_{2}\right)}{4}+\frac{\left(t_{2}-t_{1}\right)}{4} \cdot \xi\right]
\end{array}\right]
$$

Uma vez que se reduziu o número de nós de quatro para dois, haverá a necessidade de se definir dois parâmetros de deslocamento em cada nó do elemento de linha. A transformação é indicada a seguir:

Definem-se no nó le dois parâmetros, como função dos parâmetros $\chi_{1}$ e $\chi_{4}:$

$$
\begin{aligned}
& \chi_{1 \mathrm{e}}=\frac{\left(\chi_{1}+\chi_{4}\right)}{2} \\
& \chi_{1 \mathrm{e}}^{\prime}=\frac{\left(\chi_{4}-\chi_{1}\right)}{2}
\end{aligned}
$$

No nó 2e, definem-se também dois parâmetros, em função de $\chi_{2}$ e $\chi_{3}$ :

$$
\begin{aligned}
& \chi_{2 \mathrm{e}}=\frac{\left(\chi_{2}+\chi_{3}\right)}{2} \\
& \chi_{2 \mathrm{e}}^{\prime}=\frac{\left(\chi_{3}-\chi_{2}\right)}{2}
\end{aligned}
$$


Manipulando algebricamente a eq. (132) e utilizando as eqs. (163), (164), (165) e (166), escreve-se o empenamento em função dos novos parâmetros:

$$
\chi=\frac{(1-\xi)}{2} \cdot\left[\chi_{1 \mathrm{e}}+\eta \cdot \chi_{1 \mathrm{e}}^{\prime}\right]+\frac{(1+\xi)}{2} \cdot\left[\chi_{2 \mathrm{e}}+\eta \cdot \chi_{2 \mathrm{e}}^{\prime}\right]
$$

Os elementos do inverso do Jacobiano são designados como mostrado abaixo:

$$
[\mathrm{J}]^{-1}=\left[\begin{array}{ll}
\mathrm{a}_{11} & \mathrm{a}_{12} \\
\mathrm{a}_{21} & \mathrm{a}_{22}
\end{array}\right]
$$

Substituindo-se na eq. (142) o valor do Jacobiano dado pela eq. (168) e as derivadas parciais $\frac{\partial \chi}{\partial \xi}$ e $\frac{\partial \chi}{\partial \eta}$ obtidas a partir da eq. (167), chega-se a:

$$
\begin{aligned}
\left\{\begin{array}{l}
\frac{\partial \chi}{\partial \mathrm{y}} \\
\frac{\partial \chi}{\partial \mathrm{z}}
\end{array}\right\}= & {\left[\begin{array}{ll}
-\frac{1}{2} \cdot \mathrm{a}_{11} & -\frac{1}{2} \cdot \eta \cdot \mathrm{a}_{11}+\frac{(1-\xi)}{2} \cdot \mathrm{a}_{12} \\
-\frac{1}{2} \cdot \mathrm{a}_{21} & -\frac{1}{2} \cdot \eta \cdot \mathrm{a}_{21}+\frac{(1-\xi)}{2} \cdot a_{22}
\end{array}\right] \cdot\left\{\begin{array}{l}
\chi_{1 \mathrm{e}} \\
\chi_{1 \mathrm{e}}^{\prime}
\end{array}\right\}+} \\
& +\left[\begin{array}{ll}
\frac{1}{2} \cdot \mathrm{a}_{11} & \frac{1}{2} \cdot \eta \cdot \mathrm{a}_{11}+\frac{(1+\xi)}{2} \cdot \mathrm{a}_{12} \\
\frac{1}{2} \cdot \mathrm{a}_{21} & \frac{1}{2} \cdot \eta \cdot \mathrm{a}_{21}+\frac{(1+\xi)}{2} \cdot a_{22}
\end{array}\right] \cdot\left\{\begin{array}{l}
\chi_{2 \mathrm{e}} \\
\chi_{2 \mathrm{e}}^{\prime}
\end{array}\right\}
\end{aligned}
$$

Chamando-se na primeira matriz:

$$
\begin{array}{ll}
\mathrm{A}_{1}=-\frac{1}{2} \cdot \mathrm{a}_{11} & \mathrm{~A}_{1}^{\prime}=-\frac{1}{2} \cdot \eta \cdot \mathrm{a}_{11}+\frac{(1-\xi)}{2} \cdot \mathrm{a}_{12} \\
\mathrm{~B}_{1}=-\frac{1}{2} \cdot \mathrm{a}_{21} & \mathrm{~B}_{1}^{\prime}=-\frac{1}{2} \cdot \eta \cdot \mathrm{a}_{21}+\frac{(1-\xi)}{2} \cdot \mathrm{a}_{22}
\end{array}
$$

E na segunda matriz:
$\mathrm{A}_{2}=\frac{1}{2} \cdot \mathrm{a}_{11}$
$\mathrm{A}_{2}^{\prime}=\frac{1}{2} \cdot \eta \cdot \mathrm{a}_{11}+\frac{(1+\xi)}{2} \cdot \mathrm{a}_{12}$
$\mathrm{B}_{2}=\frac{1}{2} \cdot \mathrm{a}_{21}$
$\mathrm{B}_{2}^{\prime}=\frac{1}{2} \cdot \eta \cdot \mathrm{a}_{21}+\frac{(1+\xi)}{2} \cdot \mathrm{a}_{22}$

Os termos da matriz de rigidez do elemento de linha serão dados por:

$\mathrm{k}_{\mathrm{ij}}=\int_{-1-1}^{1} \int_{1}^{1}\left[\begin{array}{ll}A_{i} \cdot A_{j}+B_{i} \cdot B_{j} & A_{i} \cdot A_{j}^{\prime}+B_{i} \cdot B_{j}^{\prime} \\ A_{i}^{\prime} \cdot A_{j}+B_{i}^{\prime} \cdot B_{j} & A_{i}^{\prime} \cdot A_{j}^{\prime}+B_{i}^{\prime} \cdot B_{j}^{\prime}\end{array}\right] \cdot \operatorname{det} J d \xi d \eta$ 
em que $i=1,2 ; j=1,2$.

O vetor de forças nodais dos elementos de linha será dado por:

$$
\mathrm{p}_{\mathrm{i}}=\int_{-1}^{1} \int_{-1}^{1}\left\{\begin{array}{l}
\mathrm{S}_{1} \cdot \mathrm{A}_{\mathrm{i}}+\mathrm{S}_{2} \cdot \mathrm{B}_{\mathrm{i}}+2 \cdot(1+\mathrm{v}) \cdot \mathrm{N}_{\mathrm{i}} \cdot\left(\mathrm{y} \cdot \mathrm{k}_{\mathrm{y}}+\mathrm{z} \cdot \mathrm{k}_{\mathrm{z}}\right) \\
\mathrm{S}_{1} \cdot \mathrm{A}_{\mathrm{i}}^{\prime}+\mathrm{S}_{2} \cdot \mathrm{B}_{\mathrm{i}}^{\prime}+2 \cdot(1+\mathrm{v}) \cdot \mathrm{N}_{\mathrm{i}} \cdot\left(\mathrm{y} \cdot \mathrm{k}_{\mathrm{y}}+\mathrm{z} \cdot \mathrm{k}_{\mathrm{z}}\right)
\end{array}\right\} \cdot \operatorname{det} \mathrm{J} \mathrm{d} \xi \mathrm{d} \eta
$$

em que $\mathrm{i}=1,2$ e $\mathrm{S}_{1}$ e $\mathrm{S}_{2}$ são dados na eq. (128) e (129).

Dispondo-se agora da matriz de rigidez do elemento de linha, monta-se a matriz de rigidez da seção transversal, de acordo com as conectividades dos elementos. O mesmo se faz em relação ao vetor de forças nodais.

O sistema linear obtido é indeterminado. Para que se torne possível e determinado é preciso que se atribua um valor arbitrário a qualquer dos parâmetros $\chi$ dos nós (atribuir um valor arbitrário a qualquer dos parâmetros $\chi^{\prime}$ faz com que o sistema continue indeterminado).

Resolve-se, então, o sistema linear e tem-se a solução. O passo seguinte é o cálculo dos fatores de forma nas direções y e $\mathrm{z}$, que também é feito pelos dois processos descritos anteriormente:

1) Processo de imposição de condições de contorno nos deslocamentos.

Deve-se resolver o sistema linear duas vezes. Na primeira aplica-se carregamento $\mathrm{V}_{\mathrm{y}}$ arbitrário, mantendo-se $\mathrm{V}_{\mathrm{z}}=0$. Com o vetor de deslocamentos encontrado, calculam-se:

$$
\begin{aligned}
& \alpha_{\mathrm{yy}}=\frac{1}{\mathrm{I}_{\mathrm{z}}} \cdot\left\{\frac{\mathrm{G} \cdot \mathrm{A}}{\mathrm{V}_{\mathrm{y}}} \cdot\left[\sum_{\mathrm{k}=1}^{\mathrm{ne}} \int_{-1}^{1} \int_{-1}^{1} \chi^{\mathrm{k}} \cdot \mathrm{y}_{\mathrm{k}} \cdot \operatorname{det} \mathrm{Jd} \xi \mathrm{d} \eta\right]-\frac{\mathrm{V}}{4 \cdot(1+\mathrm{v})} \cdot\left(\mathrm{I}_{\mathrm{z}}-\mathrm{I}_{\mathrm{y}}\right)\right\} \\
& \alpha_{\mathrm{yz}}=\frac{1}{\mathrm{I}_{\mathrm{y}}} \cdot\left\{-\frac{\mathrm{G} \cdot \mathrm{A}}{\mathrm{V}_{\mathrm{y}}} \cdot\left[\sum_{\mathrm{k}=1}^{\mathrm{ne}} \int_{-1}^{1} \int_{-1}^{1} \chi^{\mathrm{k}} \cdot \mathrm{z}_{\mathrm{k}} \cdot \operatorname{det} \mathrm{J} \mathrm{d} \xi \mathrm{d} \eta\right]\right\}
\end{aligned}
$$

Da segunda vez, aplica-se $V_{z}$ arbitrário, mantendo-se $V_{y}=0$ e com o vetor de deslocamentos obtidos, calculam-se:

$$
\begin{aligned}
& \alpha_{z y}=\frac{1}{I_{z}} \cdot\left\{-\frac{G \cdot A}{V_{z}} \cdot\left[\sum_{k=1}^{n e} \int_{-1}^{1} \int_{-1}^{1} \chi^{k} \cdot y_{k} \cdot \operatorname{det} J d \xi d \eta\right]\right\} \\
& \alpha_{z z}=\frac{1}{I_{y}} \cdot\left\{\frac{G \cdot A}{V_{z}} \cdot\left[\sum_{k=1}^{n e} \int_{-1}^{1} \int_{-1}^{1} \chi^{k} \cdot z_{k} \cdot \operatorname{det} J d \xi d \eta\right]+\frac{v}{4 \cdot(1+v)} \cdot\left(I_{z}-I_{y}\right)\right\}
\end{aligned}
$$


sendo:

$\mathrm{n}_{\mathrm{e}}$ : número de elementos em que a seção transversal foi dividida.

$\mathrm{y}_{\mathrm{k}} \quad \mathrm{e} \quad \mathrm{z}_{\mathrm{k}}$ dados pelas eqs. (160) e (161)

$\chi^{\mathrm{k}}$ é dado pela eq. (167)

2) Processo de igualdade da energia de deformação.

O sistema também deve ser resolvido duas vezes. $\mathrm{Na}$ primeira com carregamento arbitrário $\mathrm{V}_{\mathrm{y}}$ e fazendo $\mathrm{V}_{\mathrm{z}}=0$, calcula-se o vetor de deslocamentos $\chi$. Multiplicando esse vetor por $\mathrm{I}_{\mathrm{y}} / \mathrm{k}_{\mathrm{y}}$, obtém-se o vetor $\chi_{\mathrm{y}}$. Com estes valores, calculam-se em cada um dos $\mathrm{k}$ elementos:

$$
\begin{aligned}
& \beta_{\mathrm{yy}}{ }^{\mathrm{k}}=\frac{\partial \chi_{\mathrm{y}}{ }^{\mathrm{k}}}{\partial \mathrm{y}}-\frac{1}{2} \cdot \mathrm{I}_{\mathrm{y}} \cdot\left(\mathrm{y}_{\mathrm{k}}{ }^{2}-\mathrm{z}_{\mathrm{k}}{ }^{2}\right) \\
& \beta_{\mathrm{yz}}=\frac{\partial \chi_{\mathrm{y}}{ }^{\mathrm{k}}}{\partial \mathrm{z}}-\mathrm{I}_{\mathrm{y}} \cdot \mathrm{y}_{\mathrm{k}} \cdot \mathrm{z}_{\mathrm{k}}
\end{aligned}
$$

Na segunda resolução do sistema, aplica-se $V_{z}$ arbitrário e $V_{y}=0$. O vetor de deslocamentos $\chi$ obtido é multiplicado por $\mathrm{I}_{\mathrm{z}} / \mathrm{k}_{\mathrm{z}}$, obtendo-se o vetor $\chi_{\mathrm{z}}$. Calculam-se então em cada elemento $\mathrm{k}$ :

$$
\begin{aligned}
& \beta_{z y}{ }^{k}=\frac{\partial \chi_{z}{ }^{k}}{\partial y}-I_{z} \cdot y_{k} \cdot z_{k} \\
& \beta_{z z}{ }^{k}=\frac{\partial \chi_{z}{ }^{k}}{\partial z}-\frac{1}{2} \cdot I_{z} \cdot\left(z_{k}{ }^{2}-y_{k}{ }^{2}\right)
\end{aligned}
$$

O valor dos fatores de forma é dado então pelo cálculo:

$$
\begin{aligned}
& \alpha_{\mathrm{yy}}=\frac{\mathrm{A}}{4 \cdot(1+v)^{2} \cdot \mathrm{I}_{\mathrm{y}} \cdot \mathrm{I}_{\mathrm{z}}} \cdot\left[\sum_{\mathrm{k}=1}^{\mathrm{n}_{\mathrm{e}}} \int_{-1-1}^{1} \int_{-1}^{1}\left(\beta_{\mathrm{yy}}{ }^{\mathrm{k}}+\beta_{\mathrm{yz}}{ }^{2}\right) \cdot \operatorname{det} \mathrm{Jd} \xi \mathrm{d} \eta\right] \\
& \alpha_{\mathrm{yz}}=\alpha_{\mathrm{zy}}=-\frac{\mathrm{A}}{4 \cdot(1+v)^{2} \cdot \mathrm{I}_{\mathrm{y}} \cdot \mathrm{I}_{\mathrm{z}}} \cdot\left[\sum_{\mathrm{k}=1}^{\mathrm{n}_{\mathrm{e}}} \int_{-1}^{1} \int_{-1}^{1}\left(\beta_{\mathrm{yy}}{ }^{\mathrm{k}} \cdot \beta_{\mathrm{zy}}{ }^{\mathrm{k}}+\beta_{\mathrm{yz}}{ }^{\mathrm{k}} \cdot \beta_{\mathrm{zz}}\right) \cdot \operatorname{det} \mathrm{J} \mathrm{d} \xi \mathrm{d} \eta\right] \\
& \alpha_{\mathrm{zz}}=\frac{\mathrm{A}}{4 \cdot(1+v)^{2} \cdot \mathrm{I}_{\mathrm{y}} \cdot \mathrm{I}_{\mathrm{z}}} \cdot\left[\sum_{\mathrm{k}=1}^{\mathrm{n}_{\mathrm{e}}} \int_{-1}^{1} \int_{-1}^{1}\left(\beta_{\mathrm{zy}}{ }^{\mathrm{k}}+\beta_{\mathrm{zz}}{ }^{2}\right) \cdot \operatorname{det} \mathrm{Jd} \xi \mathrm{d} \eta\right]
\end{aligned}
$$


Para o cálculo das tensões de cisalhamento, o sistema linear só precisa ser resolvido uma única vez, podendo as forças $\mathrm{V}_{\mathrm{y}}$ e $\mathrm{V}_{\mathrm{z}}$ serem aplicadas ao mesmo tempo.

A expressão das tensões de cisalhamento em qualquer ponto no interior de um elemento k é dada por:

$$
\begin{aligned}
& \tau_{\mathrm{xy}}{ }^{\mathrm{k}}=\mathrm{G} \cdot\left\{\frac{\partial \chi^{\mathrm{k}}}{\partial \mathrm{y}}-\mathrm{v} \cdot\left[\frac{1}{2} \cdot \mathrm{k}_{\mathrm{y}} \cdot\left(\mathrm{y}_{\mathrm{k}}{ }^{2}-\mathrm{z}_{\mathrm{k}}{ }^{2}\right)+\mathrm{y}_{\mathrm{k}} \cdot \mathrm{z}_{\mathrm{k}} \cdot \mathrm{k}_{\mathrm{z}}\right]\right\} \\
& \tau_{\mathrm{xz}}{ }^{\mathrm{k}}=\mathrm{G} \cdot\left\{\frac{\partial \chi^{\mathrm{k}}}{\partial \mathrm{z}}-v \cdot\left[\mathrm{y}_{\mathrm{k}} \cdot \mathrm{z}_{\mathrm{k}} \cdot \mathrm{k}_{\mathrm{y}}-\frac{1}{2} \cdot \mathrm{k}_{\mathrm{z}} \cdot\left(\mathrm{y}_{\mathrm{k}}{ }^{2}-\mathrm{z}_{\mathrm{k}}{ }^{2}\right)\right]\right\}
\end{aligned}
$$

sendo $y_{k}$ e $z_{k}$ dados pelas eqs. (160) e (161) e $\chi^{k}$ é dado pela eq. (167).

\section{4 - Elementos de Timoshenko e respectivas matrizes de rigidez}

Nos itens anteriores, apresentou-se a formulação necessária ao cálculo de fatores de forma por meio analítico ou numérico (MEF), o que é uma condição para o emprego da teoria de Timoshenko. Apresentam-se a seguir matrizes de rigidez de dois elementos de Timoshenko citados no capítulo 2, as quais foram utilizadas nas alterações do CEASO 01.

1) Matriz de rigidez desenvolvida por ARCHER (1965) e SEVERN (1970), que é a mais freqüentemente apresentada na literatura, como em GERE \& WEAVER (1965) e PRZEMIENIECKI (1968), escrita a seguir para a disposição de coordenadas indicadas na figura 16.

$$
[\mathrm{K}]_{\mathrm{A}}=\left[\begin{array}{cccc}
\frac{12 \cdot \mathrm{E} \cdot \mathrm{I}}{\mathrm{L}^{3}} \cdot \mathrm{f}_{2} & \frac{6 \cdot \mathrm{E} \cdot \mathrm{I}}{\mathrm{L}^{2}} \cdot \mathrm{f}_{2} & -\frac{12 \cdot \mathrm{E} \cdot \mathrm{I}}{\mathrm{L}^{3}} \cdot \mathrm{f}_{2} & \frac{6 \cdot \mathrm{E} \cdot \mathrm{I}}{\mathrm{L}^{2}} \cdot \mathrm{f}_{2} \\
\frac{6 \cdot \mathrm{E} \cdot \mathrm{I}}{\mathrm{L}^{2}} \cdot \mathrm{f}_{2} & \frac{4 \cdot \mathrm{E} \cdot \mathrm{I}}{\mathrm{L}} \cdot \mathrm{f}_{2} \cdot \mathrm{f}_{4} & -\frac{6 \cdot \mathrm{E} \cdot \mathrm{I}}{\mathrm{L}^{2}} \cdot \mathrm{f}_{2} & \frac{2 \cdot \mathrm{E} \cdot \mathrm{I}}{\mathrm{L}} \cdot \mathrm{f}_{2} \cdot \mathrm{f}_{3} \\
-\frac{12 \cdot \mathrm{E} \cdot \mathrm{I}}{\mathrm{L}^{3}} \cdot \mathrm{f}_{2} & -\frac{6 \cdot \mathrm{E} \cdot \mathrm{I}}{\mathrm{L}^{2}} \cdot \mathrm{f}_{2} & \frac{12 \cdot \mathrm{E} \cdot \mathrm{I}}{\mathrm{L}^{3}} \cdot \mathrm{f}_{2} & -\frac{6 \cdot \mathrm{E} \cdot \mathrm{I}}{\mathrm{L}^{2}} \cdot \mathrm{f}_{2} \\
\frac{6 \cdot \mathrm{E} \cdot \mathrm{I}}{\mathrm{L}^{2}} \cdot \mathrm{f}_{2} & \frac{2 \cdot \mathrm{E} \cdot \mathrm{I}}{\mathrm{L}} \cdot \mathrm{f}_{2} \cdot \mathrm{f}_{3} & -\frac{6 \cdot \mathrm{E} \cdot \mathrm{I}}{\mathrm{L}^{2}} \cdot \mathrm{f}_{2} & \frac{4 \cdot \mathrm{E} \cdot \mathrm{I}}{\mathrm{L}} \cdot \mathrm{f}_{2} \cdot \mathrm{f}_{4}
\end{array}\right]
$$

Os parâmetros $\mathrm{f}_{2}, \mathrm{f}_{3}$ e $\mathrm{f}_{4}$ têm a seguinte definição: 


$$
\begin{aligned}
& \mathrm{f}_{2}=\frac{1}{1+2 \cdot \mathrm{g}} \\
& \mathrm{f}_{3}=1-\mathrm{g} \\
& \mathrm{f}_{4}=1+\frac{\mathrm{g}}{2}
\end{aligned}
$$

sendo $g=\frac{6 \cdot E \cdot I \cdot \alpha}{L^{2} \cdot G \cdot A}$

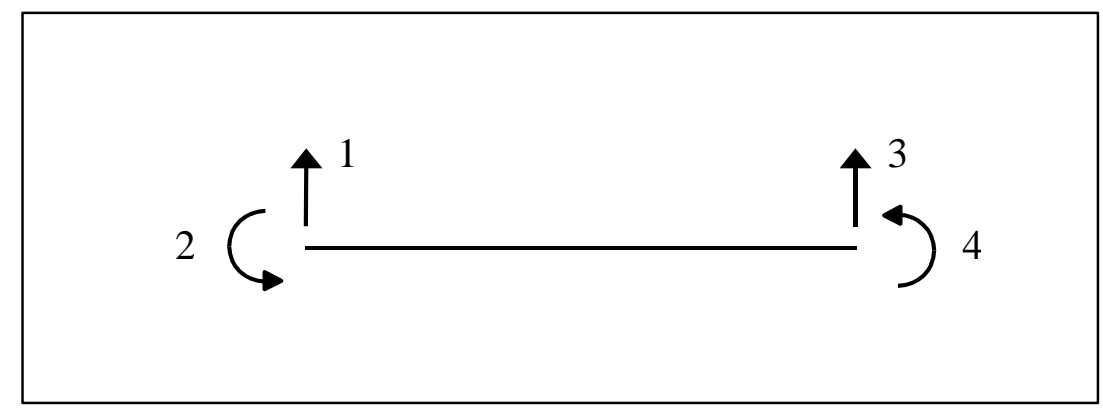

Figura 16 - Sistema de coordenadas - matriz de ARCHER

2) Matriz apresentada por PILKEY et al (1995), considerando a interdependência da flexão em torno dos eixos principais de inércia, que ocorre quando a seção é assimétrica.

A matriz apresentada corresponde à seguinte ordenação de coordenadas de deslocamentos:

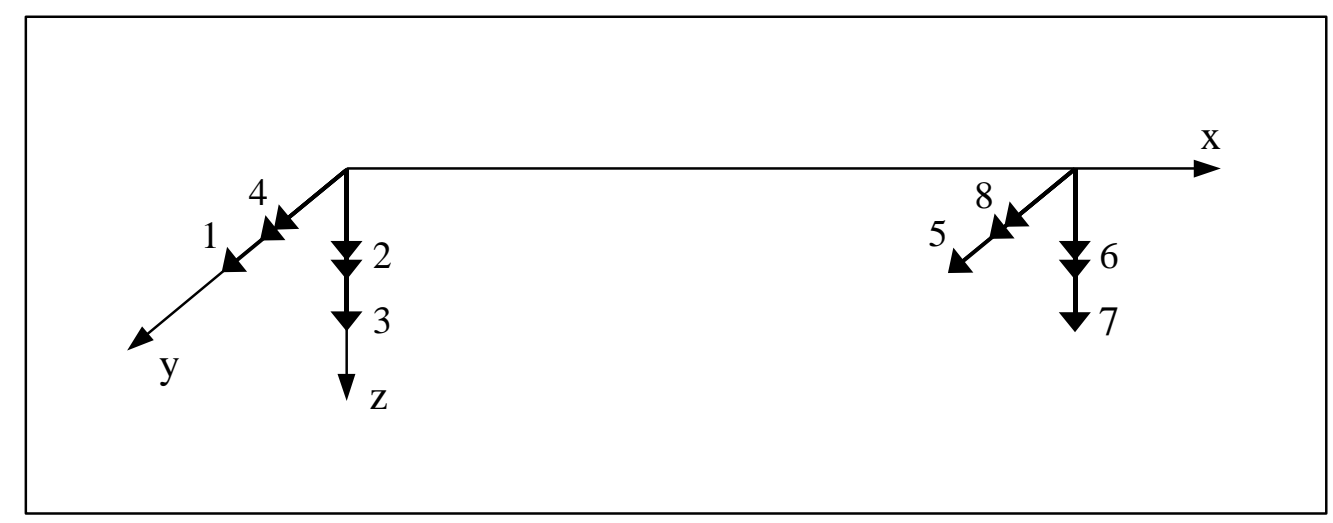

Figura 17 - Sistema de coordenadas - Matriz de PILKEY et al. 


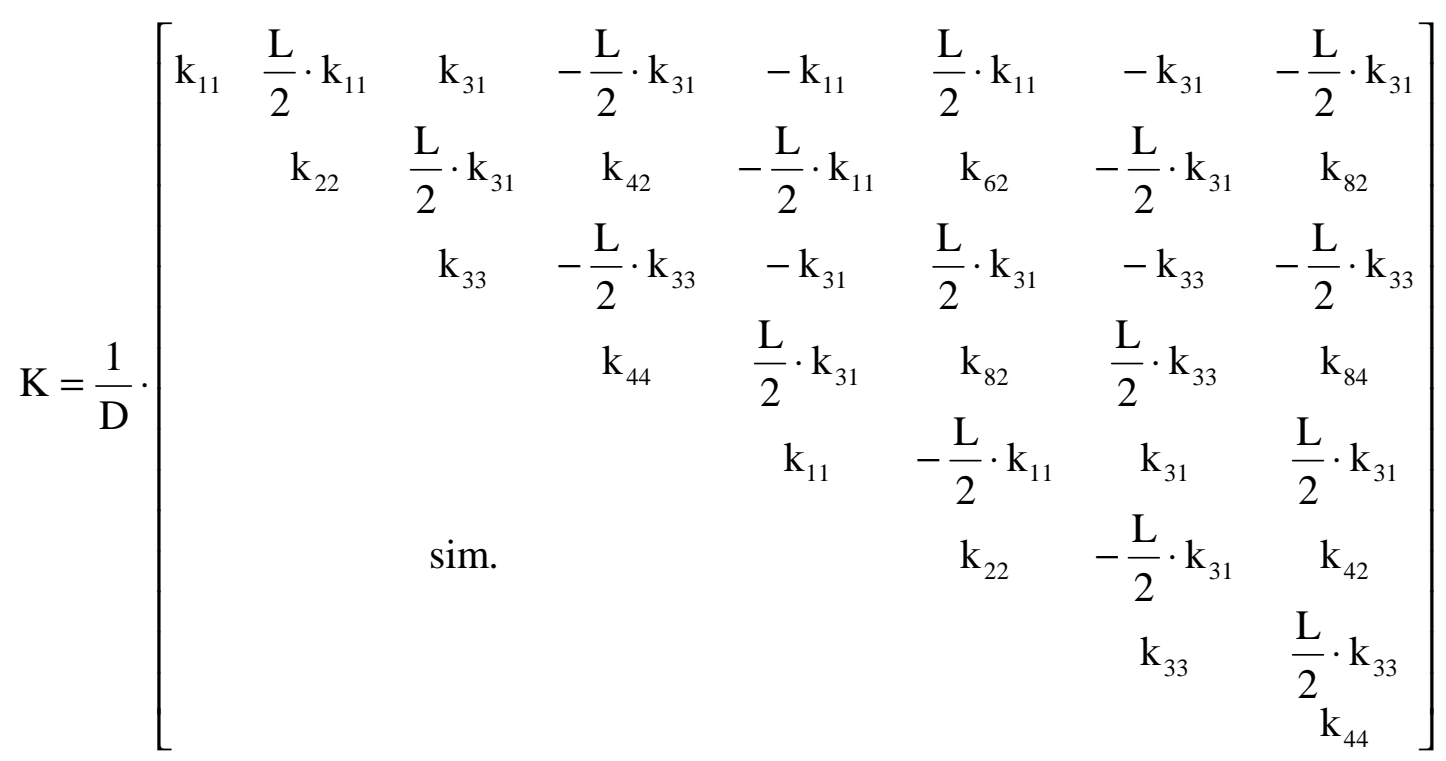

\section{Sendo:}

$$
\begin{aligned}
& D=L \cdot\left[b_{y} \cdot b_{z} \cdot L^{4}+12 \cdot\left(a_{y y} \cdot b_{y}+a_{z z} \cdot b_{z}\right) \cdot L^{2}+144 \cdot\left(a_{y y} \cdot a_{z z}-a_{y z}{ }^{2}\right)\right] \\
& a_{i j}=\frac{\alpha_{i j}}{G \cdot A}, \quad i, j=y, z \\
& b_{i}=\frac{1}{E \cdot I_{i}}, \quad i=y, z
\end{aligned}
$$

os termos da matriz são:

$$
\begin{aligned}
& \mathrm{k}_{11}=12 \cdot\left(\mathrm{b}_{\mathrm{y}} \cdot \mathrm{L}^{2}+12 \cdot \mathrm{a}_{\mathrm{zz}}\right) \\
& \mathrm{k}_{31}=-144 \cdot \mathrm{a}_{\mathrm{yz}} \\
& \mathrm{k}_{33}=12 \cdot\left(\mathrm{b}_{\mathrm{z}} \cdot \mathrm{L}^{2}+12 \cdot \mathrm{a}_{\mathrm{yy}}\right) \\
& \mathrm{k}_{22}=4 \cdot\left[\mathrm{b}_{\mathrm{y}} \cdot \mathrm{b}_{\mathrm{z}} \cdot \mathrm{L}^{4}+3 \cdot\left(4 \cdot \mathrm{a}_{\mathrm{zz}} \cdot \mathrm{b}_{\mathrm{z}}+\mathrm{a}_{\mathrm{yy}} \cdot \mathrm{b}_{\mathrm{y}}\right) \cdot \mathrm{L}^{2}+36 \cdot\left(\mathrm{a}_{\mathrm{yy}} \cdot \mathrm{a}_{\mathrm{zz}}-\mathrm{a}_{\mathrm{yz}}{ }^{2}\right)\right] / \mathrm{b}_{\mathrm{z}} \\
& \mathrm{k}_{42}=36 \cdot \mathrm{a}_{\mathrm{yz}} \cdot \mathrm{L}^{2} \\
& \mathrm{k}_{44}=4 \cdot\left[\mathrm{b}_{\mathrm{y}} \cdot \mathrm{b}_{\mathrm{z}} \cdot \mathrm{L}^{4}+3 \cdot\left(4 \cdot \mathrm{a}_{\mathrm{yy}} \cdot \mathrm{b}_{\mathrm{y}}+\mathrm{a}_{\mathrm{zz}} \cdot \mathrm{b}_{\mathrm{z}}\right) \cdot \mathrm{L}^{2}+36 \cdot\left(\mathrm{a}_{\mathrm{yy}} \cdot \mathrm{a}_{\mathrm{zz}}-\mathrm{a}_{\mathrm{yz}}{ }^{2}\right)\right] / \mathrm{b}_{\mathrm{y}} \\
& \mathrm{k}_{62}=2 \cdot\left[\mathrm{b}_{\mathrm{y}} \cdot \mathrm{b}_{\mathrm{z}} \cdot \mathrm{L}^{4}+3 \cdot\left(4 \cdot \mathrm{a}_{\mathrm{zz}} \cdot \mathrm{b}_{\mathrm{z}}-2 \cdot \mathrm{a}_{\mathrm{yy}} \cdot \mathrm{b}_{\mathrm{y}}\right) \cdot \mathrm{L}^{2}-72 \cdot\left(\mathrm{a}_{\mathrm{yy}} \cdot \mathrm{a}_{\mathrm{zz}}-\mathrm{a}_{\mathrm{yz}}{ }^{2}\right)\right] / \mathrm{b}_{\mathrm{z}} \\
& \mathrm{k}_{82}=36 \cdot \mathrm{a}_{\mathrm{yz}} \cdot \mathrm{L}^{2} \\
& \mathrm{k}_{84}=2 \cdot\left[\mathrm{b}_{\mathrm{y}} \cdot \mathrm{b}_{\mathrm{z}} \cdot \mathrm{L}^{4}+3 \cdot\left(4 \cdot \mathrm{a}_{\mathrm{yy}} \cdot \mathrm{b}_{\mathrm{y}}-2 \cdot \mathrm{a}_{\mathrm{zz}} \cdot \mathrm{b}_{\mathrm{z}}\right) \cdot \mathrm{L}^{2}-72 \cdot\left(\mathrm{a}_{\mathrm{yy}} \cdot \mathrm{a}_{\mathrm{zz}}-\mathrm{a}_{\mathrm{yz}}{ }^{2}\right)\right] / \mathrm{b}_{\mathrm{y}}
\end{aligned}
$$




\section{5 - TEORIA DE TIMOSHENKO APLICADA AOS ELEMENTOS VERTICAIS DE CONTRAVENTAMENTO}

O programa CEASO 01 apresenta cinco tipos de elementos verticais de contraventamento. A seguir, apresenta-se o sistema de coordenadas de deslocamento de cada um deles, referido ao seu sistema de eixos cartesianos locais, assim como as alterações realizadas em suas matrizes de rigidez.

ELM - 01: apresenta apenas duas coordenadas, ou seja, translação na direção axial em cada extremidade, como mostrado na figura 18. Como não apresenta flexão, sua matriz de rigidez $\left[\mathrm{R}_{1}\right]$, apresentada abaixo, não foi modificada.

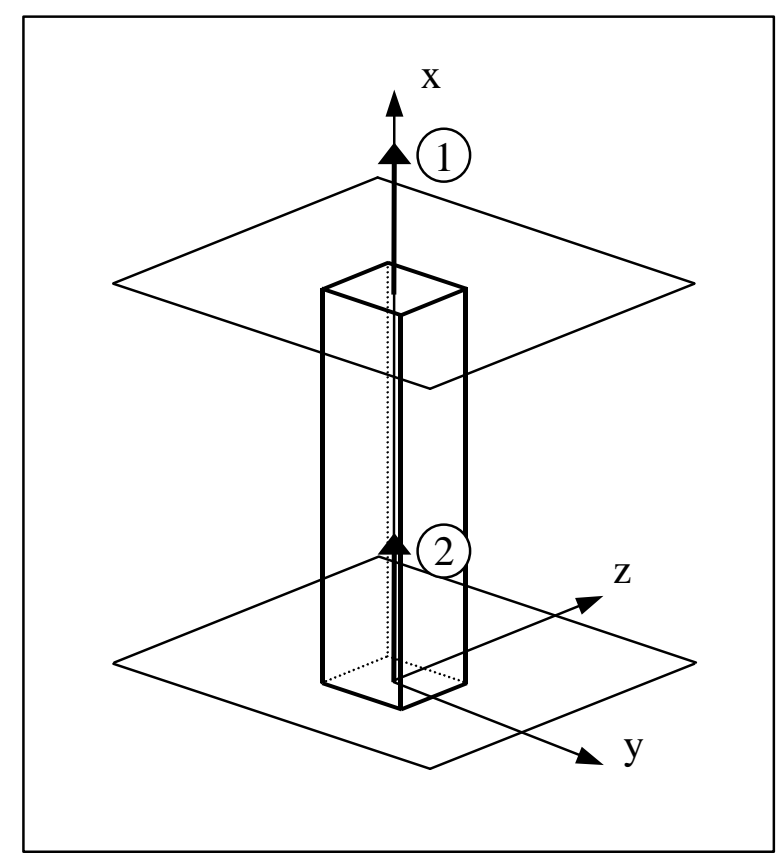

Figura 18 - Coordenadas de deslocamento do ELM - 01

$$
\left[R_{1}\right]=\left[\begin{array}{cc}
\frac{E \cdot A}{L} & -\frac{E \cdot A}{L} \\
-\frac{E \cdot A}{L} & \frac{E \cdot A}{L}
\end{array}\right]
$$


ELM - 02: é o elemento de pórtico plano, com flexão apenas no plano do painel. As suas seis coordenadas (translação na direção dos eixos x e z e rotação em torno do eixo y, em cada extremidade) são apresentadas na figura 19. As suas matrizes de rigidez, sem considerar a deformação por cortante $\left[\mathrm{R}_{2}\right]$ e com essa consideração $\left[R_{2}\right]_{C}$ são apresentadas após a figura 19. Os valores $f_{2}, f_{3}$ e $f_{4}$ são dados pelas eqs. (187), (188) e (189), apresentadas no capítulo 4.

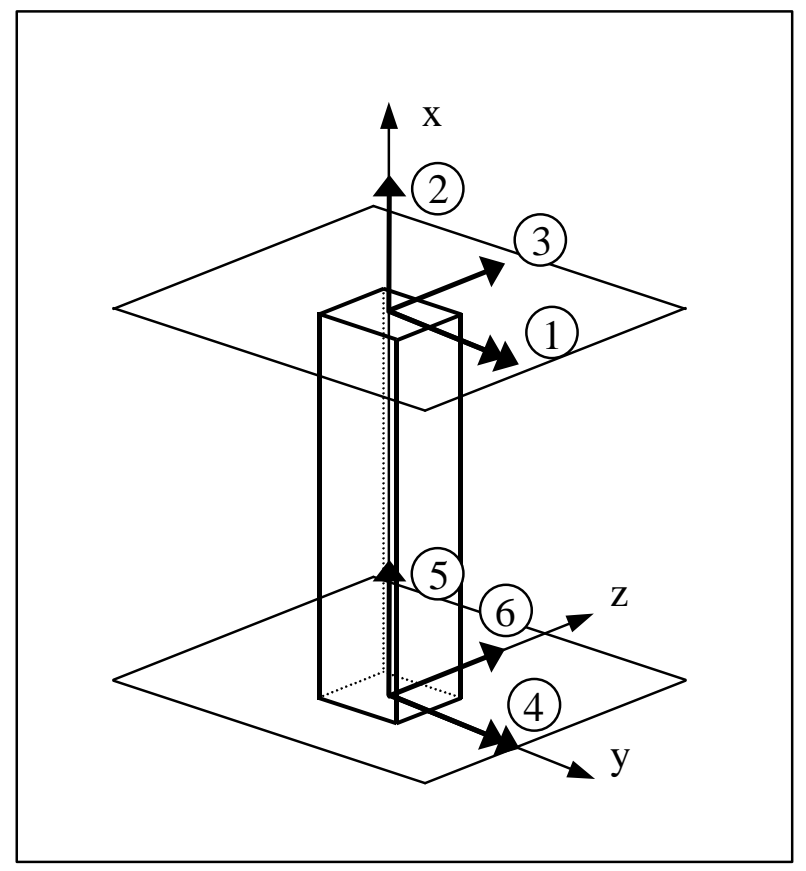

Figura 19 - Coordenadas de deslocamento do ELM - 02

$$
\left[\mathrm{R}_{2}\right]=\left[\begin{array}{ccccccc}
\frac{4 \cdot \mathrm{E} \cdot \mathrm{I}_{\mathrm{y}}}{\mathrm{L}} & 0 & \frac{6 \cdot \mathrm{E} \cdot \mathrm{I}_{\mathrm{y}}}{\mathrm{L}^{2}} & \frac{2 \cdot \mathrm{E} \cdot \mathrm{I}_{\mathrm{y}}}{\mathrm{L}} & 0 & -\frac{6 \cdot \mathrm{E} \cdot \mathrm{I}_{\mathrm{y}}}{\mathrm{L}^{2}} \\
& \frac{\mathrm{E} \cdot \mathrm{A}}{\mathrm{L}} & 0 & 0 & -\frac{\mathrm{E} \cdot \mathrm{A}}{\mathrm{L}} & 0 \\
& & \frac{12 \cdot \mathrm{E} \cdot \mathrm{I}_{\mathrm{y}}}{\mathrm{L}^{3}} & \frac{6 \cdot \mathrm{E} \cdot \mathrm{I}_{\mathrm{y}}}{\mathrm{L}^{2}} & 0 & -\frac{12 \cdot \mathrm{E} \cdot \mathrm{I}_{\mathrm{y}}}{\mathrm{L}^{3}} \\
& & \frac{4 \cdot \mathrm{E} \cdot \mathrm{I}_{\mathrm{y}}}{\mathrm{L}} & 0 & -\frac{6 \cdot \mathrm{E} \cdot \mathrm{I}_{\mathrm{y}}}{\mathrm{L}^{2}} \\
& & & \frac{\mathrm{E} \cdot \mathrm{A}}{\mathrm{L}} & 0 \\
& & & & \frac{12 \cdot \mathrm{E} \cdot \mathrm{I}_{\mathrm{y}}}{\mathrm{L}^{3}}
\end{array}\right]
$$




$$
\left[\mathrm{R}_{2}\right]_{\mathrm{C}}=\left[\begin{array}{ccccccc}
\frac{4 \cdot \mathrm{E} \cdot \mathrm{I}_{\mathrm{y}}}{\mathrm{L}} \cdot \mathrm{f}_{2} \cdot \mathrm{f}_{4} & 0 & \frac{6 \cdot \mathrm{E} \cdot \mathrm{I}_{\mathrm{y}}}{\mathrm{L}^{2}} \cdot \mathrm{f}_{2} & \frac{2 \cdot \mathrm{E} \cdot \mathrm{I}_{\mathrm{y}}}{\mathrm{L}} \cdot \mathrm{f}_{2} \cdot \mathrm{f}_{3} & 0 & -\frac{6 \cdot \mathrm{E} \cdot \mathrm{I}_{\mathrm{y}}}{\mathrm{L}^{2}} \cdot \mathrm{f}_{2} \\
& \frac{\mathrm{E} \cdot \mathrm{A}}{\mathrm{L}} & 0 & 0 & -\frac{\mathrm{E} \cdot \mathrm{A}}{\mathrm{L}} & 0 \\
& & \frac{12 \cdot \mathrm{E} \cdot \mathrm{I}_{\mathrm{y}}}{\mathrm{L}^{3}} \cdot \mathrm{f}_{2} & \frac{6 \cdot \mathrm{E} \cdot \mathrm{I}_{\mathrm{y}}}{\mathrm{L}^{2}} \cdot \mathrm{f}_{2} & 0 & -\frac{12 \cdot \mathrm{E} \cdot \mathrm{I}_{\mathrm{y}}}{\mathrm{L}^{3}} \cdot \mathrm{f}_{2} \\
& & \frac{4 \cdot \mathrm{E} \cdot \mathrm{I}_{\mathrm{y}}}{\mathrm{L}} \cdot \mathrm{f}_{2} \cdot \mathrm{f}_{4} & 0 & -\frac{6 \cdot \mathrm{E} \cdot \mathrm{I}_{\mathrm{y}}}{\mathrm{L}^{2}} \cdot \mathrm{f}_{2} \\
& & & \frac{\mathrm{E} \cdot \mathrm{A}}{\mathrm{L}} & 0 \\
& & & & \frac{12 \cdot \mathrm{E} \cdot \mathrm{I}_{\mathrm{y}}}{\mathrm{L}^{3}} \cdot \mathrm{f}_{2}
\end{array}\right]
$$

ELM - 03: é o elemento de pórtico espacial, com flexão em torno dos dois eixos principais de inércia. As suas dez coordenadas de deslocamento (translação na direção dos eixos $\mathrm{x}, \mathrm{y}$ e $\mathrm{z}$ e rotação em torno dos eixos y e $\mathrm{z}$, em cada extremidade) são apresentadas na figura 20. A sua matriz de rigidez sem considerar a deformação por cortante $\left[\mathrm{R}_{3}\right]$ é subdividida em quatro submatrizes, assim como a matriz que considera o efeito acima referido $\left[\mathrm{R}_{3}\right]_{\mathrm{C}}$. Essas submatrizes são apresentadas após a figura 20. Os parâmetros $\mathrm{f}_{2 \mathrm{y}}, \mathrm{f}_{3 \mathrm{y}}, \mathrm{f}_{4 \mathrm{y}}, \mathrm{f}_{2 \mathrm{z}}, \mathrm{f}_{3 z}$ e $\mathrm{f}_{4 z}$ são obtidos das eqs. (187), (188) e (189), bastando substituir $\mathrm{I}_{\text {por }} \mathrm{I}_{\mathrm{y}}$ ou $\mathrm{I}_{\mathrm{z}}$.

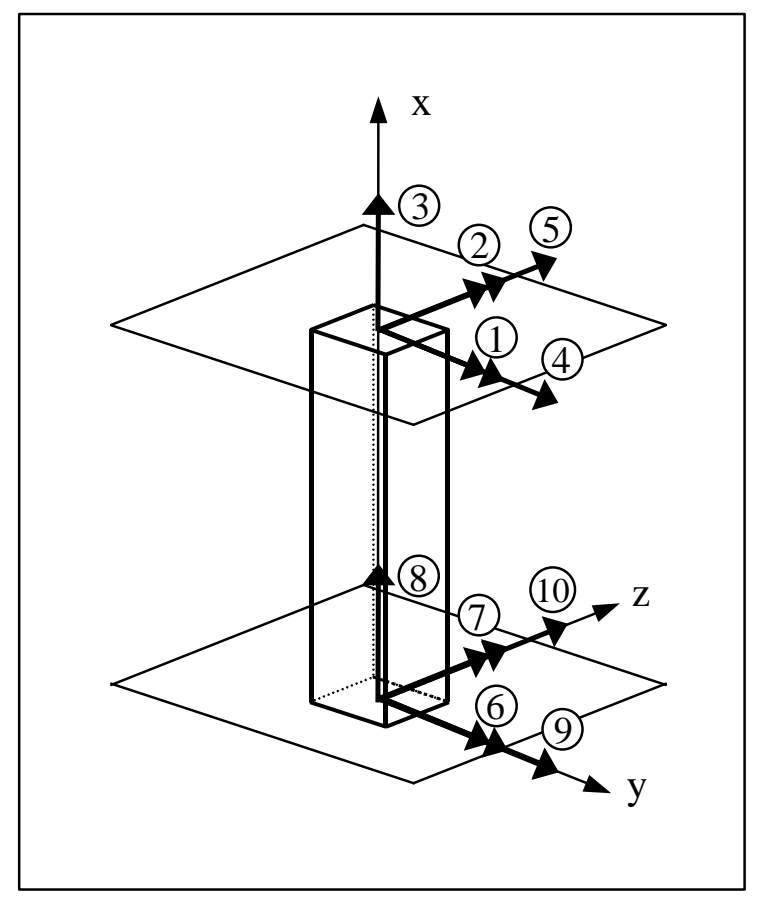

Figura 20 - Coordenadas de deslocamento do ELM - 03 
Sendo $\left[\mathrm{R}_{3}\right]=\left[\begin{array}{ll}{[\mathrm{R}]_{\mathrm{aa}}} & {[\mathrm{R}]_{\mathrm{ab}}} \\ {[\mathrm{R}]_{\mathrm{ba}}} & {[\mathrm{R}]_{\mathrm{bb}}}\end{array}\right]$ temos:

$$
[\mathrm{R}]_{\mathrm{aa}}=\left[\begin{array}{ccccc}
\frac{4 \cdot \mathrm{E} \cdot \mathrm{I}_{\mathrm{y}}}{\mathrm{L}} & 0 & 0 & 0 & \frac{6 \cdot \mathrm{E} \cdot \mathrm{I}_{\mathrm{y}}}{\mathrm{L}^{2}} \\
& \frac{4 \cdot \mathrm{E} \cdot \mathrm{I}_{\mathrm{z}}}{\mathrm{L}} & 0 & -\frac{6 \cdot \mathrm{E} \cdot \mathrm{I}_{\mathrm{z}}}{\mathrm{L}^{2}} & 0 \\
& & \frac{\mathrm{E} \cdot \mathrm{A}}{\mathrm{L}} & 0 & 0 \\
& \text { simétrica } & \frac{12 \cdot \mathrm{E} \cdot \mathrm{I}_{\mathrm{z}}}{\mathrm{L}^{3}} & 0 \\
& & & & \frac{12 \cdot \mathrm{E} \cdot \mathrm{I}_{\mathrm{y}}}{\mathrm{L}^{3}}
\end{array}\right]
$$

$$
[R]_{a b}=\left[\begin{array}{ccccc}
\frac{2 \cdot E \cdot I_{y}}{L} & 0 & 0 & 0 & -\frac{6 \cdot E \cdot I_{y}}{L^{2}} \\
0 & \frac{2 \cdot E \cdot I_{z}}{L} & 0 & \frac{6 \cdot E \cdot I_{z}}{L^{2}} & 0 \\
0 & 0 & -\frac{E \cdot A}{L} & 0 & 0 \\
0 & -\frac{6 \cdot E \cdot I_{z}}{L^{2}} & 0 & -\frac{12 \cdot E \cdot I_{z}}{L^{3}} & 0 \\
\frac{6 \cdot E \cdot I_{y}}{L^{2}} & 0 & 0 & 0 & -\frac{12 \cdot E \cdot I_{y}}{L^{3}}
\end{array}\right]
$$

$$
[R]_{b a}=\left[\begin{array}{ccccc}
\frac{2 \cdot E \cdot I_{y}}{L} & 0 & 0 & 0 & \frac{6 \cdot E \cdot I_{y}}{L^{2}} \\
0 & \frac{2 \cdot E \cdot I_{z}}{L} & 0 & -\frac{6 \cdot E \cdot I_{z}}{L^{2}} & 0 \\
0 & 0 & -\frac{E \cdot A}{L} & 0 & 0 \\
0 & \frac{6 \cdot E \cdot I_{z}}{L^{2}} & 0 & -\frac{12 \cdot E \cdot I_{z}}{L^{3}} & 0 \\
-\frac{6 \cdot E \cdot I_{y}}{L^{2}} & 0 & 0 & 0 & -\frac{12 \cdot E \cdot I_{y}}{L^{3}}
\end{array}\right]
$$




$$
[\mathrm{R}]_{\mathrm{bb}}=\left[\begin{array}{ccccc}
\frac{4 \cdot \mathrm{E} \cdot \mathrm{I}_{\mathrm{y}}}{\mathrm{L}} & 0 & 0 & 0 & -\frac{6 \cdot \mathrm{E} \cdot \mathrm{I}_{\mathrm{y}}}{\mathrm{L}^{2}} \\
& \frac{4 \cdot \mathrm{E} \cdot \mathrm{I}_{\mathrm{z}}}{\mathrm{L}} & 0 & \frac{6 \cdot \mathrm{E} \cdot \mathrm{I}_{\mathrm{z}}}{\mathrm{L}^{2}} & 0 \\
& & \frac{\mathrm{E} \cdot \mathrm{A}}{\mathrm{L}} & 0 & 0 \\
& \text { simétrica } & \frac{12 \cdot \mathrm{E} \cdot \mathrm{I}_{\mathrm{z}}}{\mathrm{L}^{3}} & 0 \\
& & & & \frac{12 \cdot \mathrm{E} \cdot \mathrm{I}_{\mathrm{y}}}{\mathrm{L}^{3}}
\end{array}\right]
$$

Sendo $\left[\mathrm{R}_{3}\right]_{\mathrm{C}}=\left[\begin{array}{ll}{[\mathrm{R}]_{\mathrm{cc}}} & {[\mathrm{R}]_{\mathrm{cd}}} \\ {[\mathrm{R}]_{\mathrm{dc}}} & {[\mathrm{R}]_{\mathrm{dd}}}\end{array}\right]$ temos:

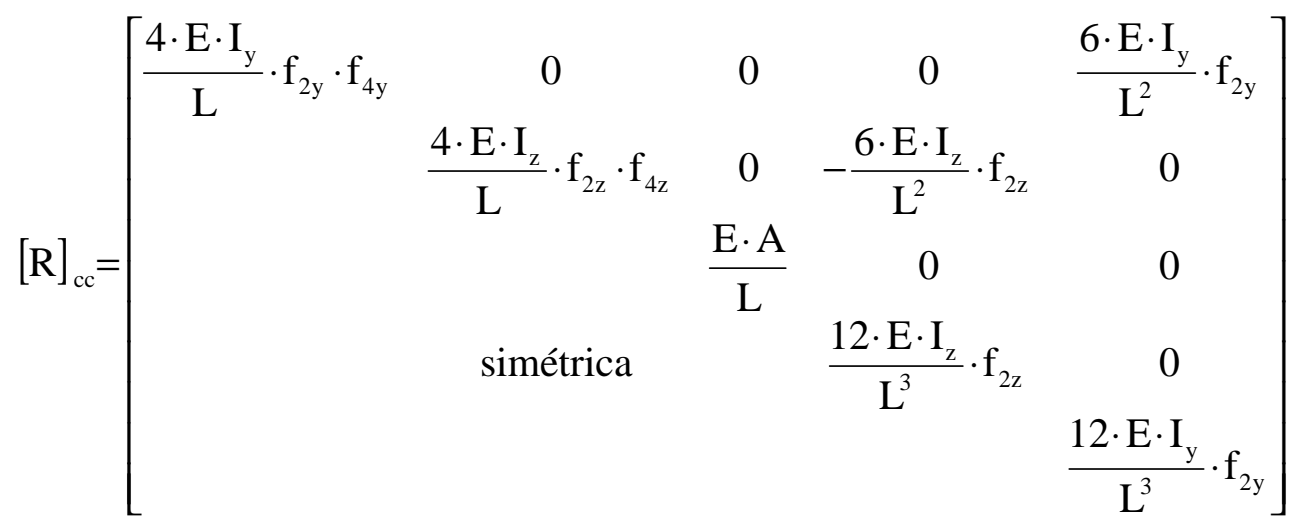

$$
[R]_{c d}=\left[\begin{array}{ccccc}
\frac{2 \cdot E \cdot I_{y}}{L} \cdot f_{2 y} \cdot f_{3 y} & 0 & 0 & 0 & -\frac{6 \cdot E \cdot I_{y}}{L^{2}} \cdot f_{2 y} \\
0 & \frac{2 \cdot E \cdot I_{z}}{L} \cdot f_{2 z} \cdot f_{3 z} & 0 & \frac{6 \cdot E \cdot I_{z}}{L^{2}} \cdot f_{2 z} & 0 \\
0 & 0 & -\frac{E \cdot A}{L} & 0 & 0 \\
0 & -\frac{6 \cdot E \cdot I_{z}}{L^{2}} \cdot f_{2 z} & 0 & -\frac{12 \cdot E \cdot I_{z}}{L^{3}} \cdot f_{2 z} & 0 \\
\frac{6 \cdot E \cdot I_{y}}{L^{2}} \cdot f_{2 y} & 0 & 0 & 0 & -\frac{12 \cdot E \cdot I_{y}}{L^{3}} \cdot f_{2 y}
\end{array}\right]
$$


$[R]_{d c}=\left[\begin{array}{ccccc}\frac{2 \cdot E \cdot I_{y}}{L} \cdot f_{2 y} \cdot f_{3 y} & 0 & 0 & 0 & \frac{6 \cdot E \cdot I_{y}}{L^{2}} \cdot f_{2 y} \\ 0 & \frac{2 \cdot E \cdot I_{z}}{L} \cdot f_{2 z} \cdot f_{3 z} & 0 & -\frac{6 \cdot E \cdot I_{z}}{L^{2}} \cdot f_{2 z} & 0 \\ 0 & 0 & -\frac{E \cdot A}{L} & 0 & 0 \\ 0 & \frac{6 \cdot E \cdot I_{z}}{L^{2}} \cdot f_{2 z} & 0 & -\frac{12 \cdot E \cdot I_{z}}{L^{3}} \cdot f_{2 z} & 0 \\ -\frac{6 \cdot E \cdot I_{y}}{L^{2}} \cdot f_{2 y} & 0 & 0 & 0 & -\frac{12 \cdot E \cdot I_{y}}{L^{3}} \cdot f_{2 y}\end{array}\right]$

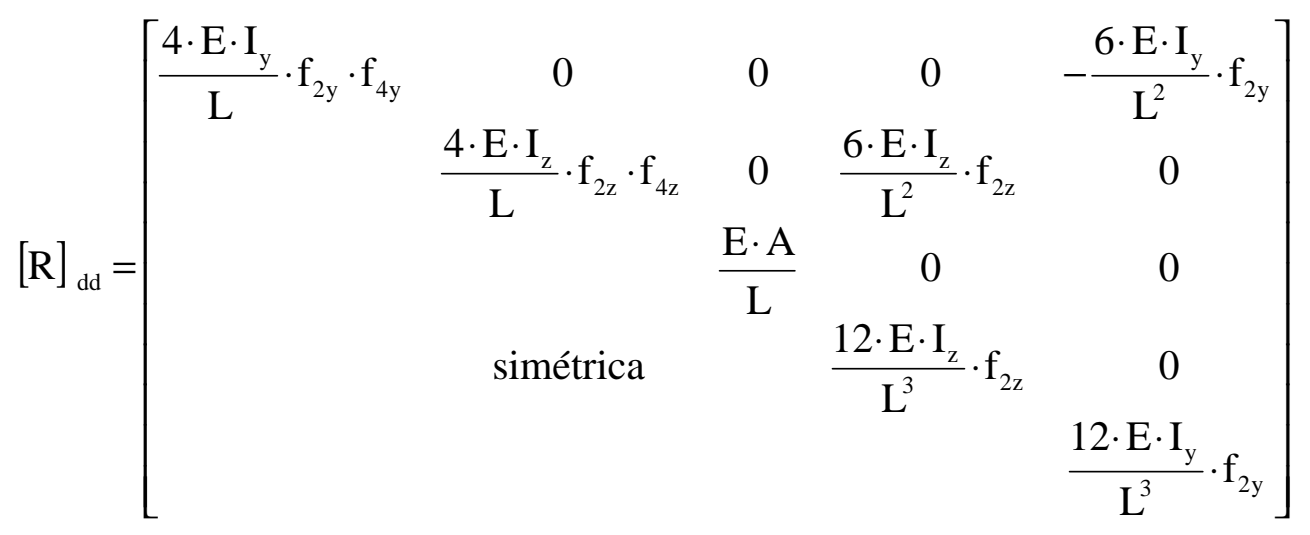

ELM - 04: apresenta duas coordenadas a mais que o ELM - 03, que são as rotações em torno do eixo $\mathrm{x}$ em cada extremidade do elemento, como se mostra na figura 21. Portanto a submatriz de flexão deste elemento, seja considerando ou não a deformação pelo esforço cortante, é idêntica à do ELM - 03.

ELM - 05: é o elemento de barra de sete coordenadas por nó desenvolvido por TARANATH (1968): translações nas direções dos eixos x, y e z e rotações em torno desses eixos, além da primeira derivada da rotação em torno do eixo $\mathrm{x}$. O sistema de coordenadas de deslocamento desse elemento é mostrado na figura 22 . No que se refere à flexão, o comportamento deste elemento é o mesmo do ELM - 03 e portanto as submatrizes de rigidez, com ou sem efeito do cortante, são as apresentadas anteriormente para aquele elemento. No caso da consideração de deformação pelo cortante, existe a opção de utilizar a matriz dada por PILKEY et al 
(1995), que considera o acoplamento da flexão em torno dos dois eixos principais e é apresentada no capítulo 4 , no item 4.4.

A submatriz referente às coordenadas da teoria de flexo-torção de Vlassov é indicada a seguir, sendo a primeira linha (ou coluna) relativa à coordenada 6 , a segunda linha, à coordenada 7, a terceira linha, à coordenada 13 e a quarta linha, à coordenada 14. Esta submatriz é desacoplada da submatriz de flexão e denominada $[\mathrm{R}]_{5 \mathrm{ft}}$.

$$
[R]_{5 \mathrm{ft}}=\gamma \cdot\left[\begin{array}{cccc}
\alpha \cdot \mathrm{sh} & 1-\mathrm{ch} & -\alpha \cdot \mathrm{sh} & 1-\mathrm{ch} \\
& \mathrm{L} \cdot \mathrm{ch}-\frac{\mathrm{sh}}{\alpha} & \mathrm{ch}-1 & \frac{\mathrm{sh}}{\alpha}-\mathrm{L} \\
& & \alpha \cdot \mathrm{sh} & \mathrm{ch}-1 \\
& \mathrm{sim} . & & \mathrm{L} \cdot \mathrm{ch}-\frac{\mathrm{sh}}{\alpha}
\end{array}\right]
$$

Sendo L o comprimento do elemento de núcleo, $\mathrm{I}_{\mathrm{x}} \mathrm{o}$ momento de inércia à torção e $I_{\omega}$ o momento setorial de inércia, as variáveis presentes na matriz acima são as seguintes:

$$
\begin{aligned}
& \alpha=\sqrt{\frac{G \cdot I_{x}}{E \cdot I_{\omega}}} \\
& \operatorname{sh}=\operatorname{senh}(\alpha \cdot L) \\
& \operatorname{ch}=\operatorname{coh}(\alpha \cdot L) \\
& \gamma=\frac{G \cdot I_{x}}{2-2 \cdot \operatorname{ch}+L \cdot \alpha \cdot \operatorname{sh}}
\end{aligned}
$$




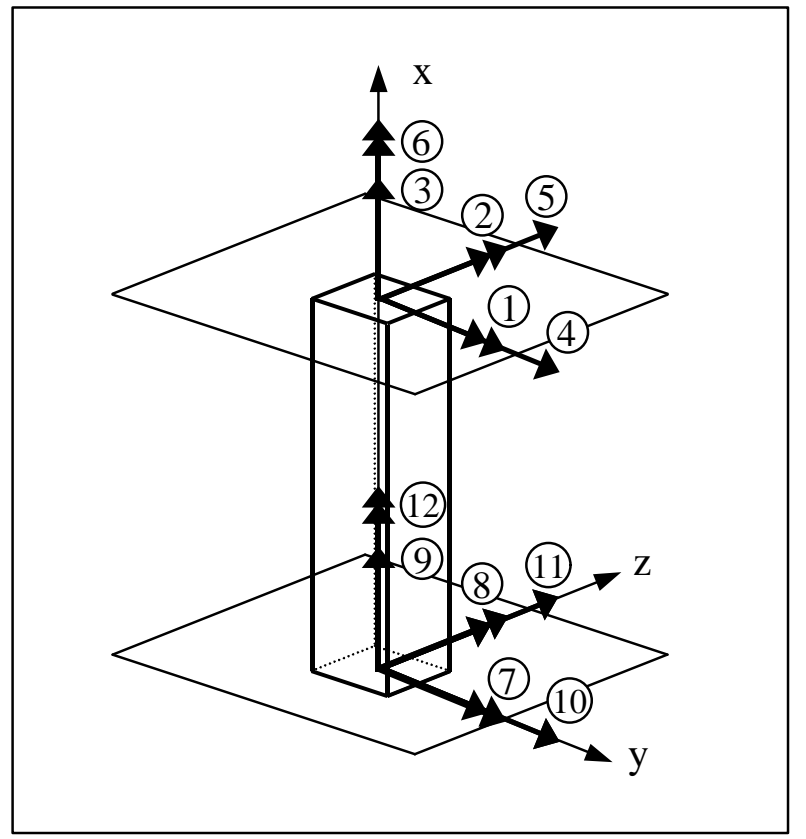

Figura 21 - Coordenadas de deslocamento do ELM - 04

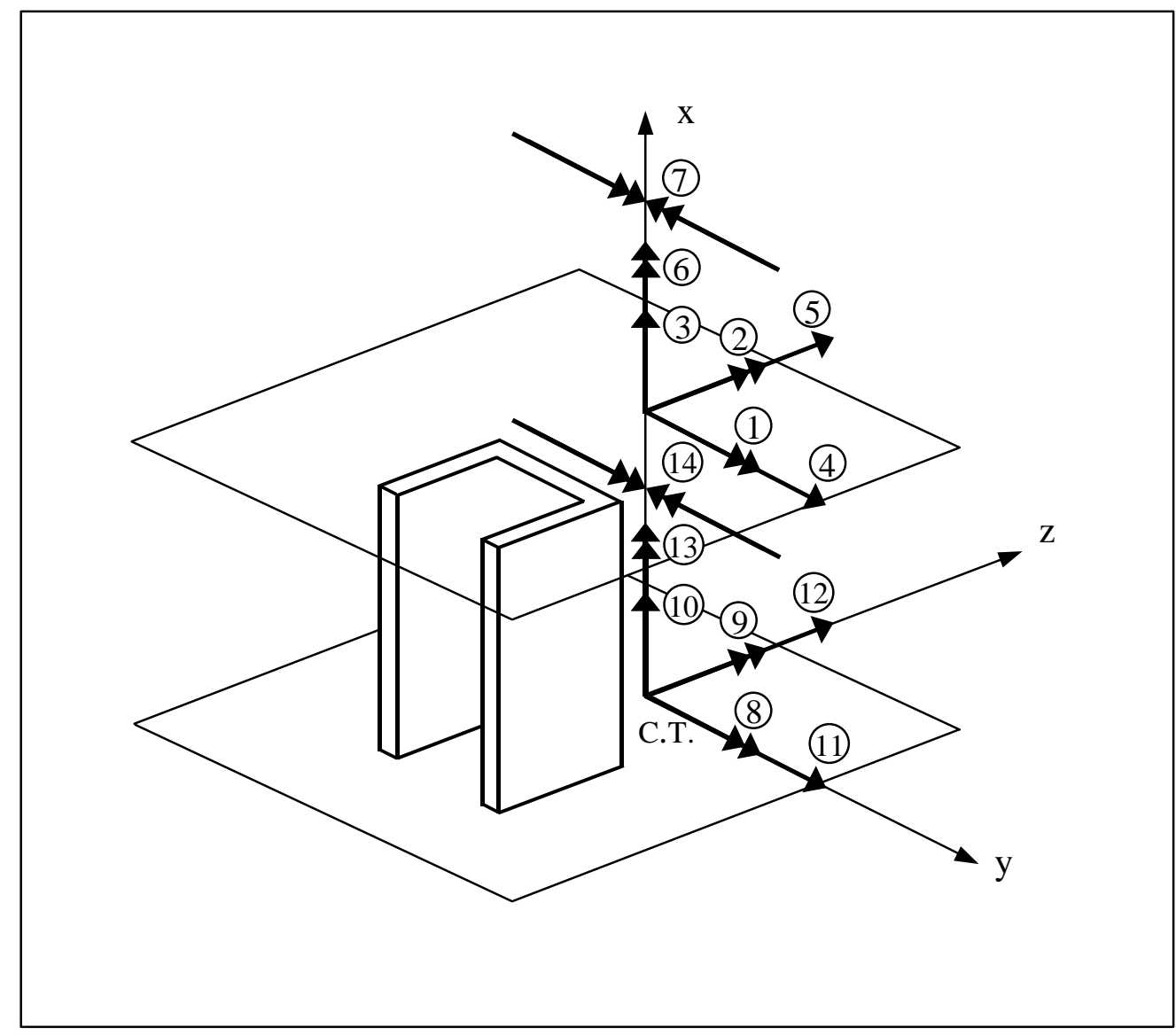

Figura 22 - Coordenadas de deslocamento do ELM - 05 


\section{6 - RESULTADOS OBTIDOS}

\section{1 - Exemplo 1}

O objetivo deste exemplo é aferir os resultados obtidos com o programa CEASO 01 modificado, comparando-os com os de trabalhos já realizados.

Para isso, utiliza-se um exemplo extraído de PLAIS (1998), que consiste numa viga de seção transversal em I engastada numa extremidade, com aplicação de força concentrada na extremidade livre de valor $\mathrm{P}=100 \mathrm{kN}$, conforme mostrado na figura 23 .

A viga em questão é subdividida em vinte elementos e apresenta a seção transversal mostrada na figura 24. Para a seção I apresentada, adotam-se módulo de elasticidade longitudinal $E=21.000 \mathrm{kN} / \mathrm{cm}^{2}$ e coeficiente de Poisson $v=0,3$. No cálculo do fator de forma, utiliza-se a expressão simplificada $\alpha=\mathrm{A} / \mathrm{A}_{\mathrm{w}}$ (sendo $\mathrm{A}$ a área da seção transversal e $\mathrm{A}_{\mathrm{w}}$ a área da alma), proposta por diversos autores, entre os quais GERE \& WEAVER (1965), que resulta no valor $\alpha=3,22222$. O fator de forma calculado pelo processo geométrico foi $\alpha=3,25284$ e pelo energético, $\alpha=3,27637$.

A tabela 2 apresenta os valores do deslocamento sob a carga $\mathrm{P}$ para uma variação do comprimento da viga desde $L=25 \mathrm{~cm}$ até $\mathrm{L}=500 \mathrm{~cm}$.

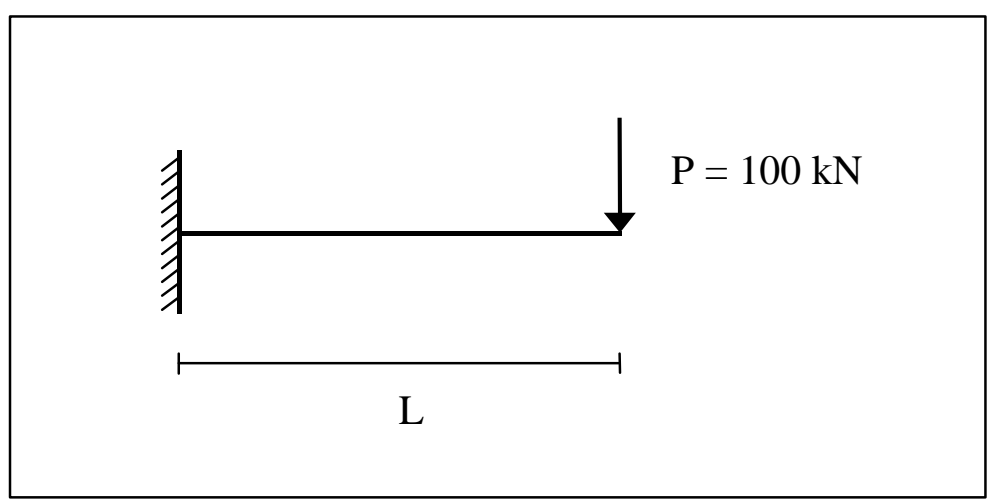

Figura 23 - Exemplo de carregamento extraído de PLAIS 
No programa CEASO 01, esta estrutura é modelada como um edifício de vinte pavimentos que consiste em apenas um elemento de núcleo, com as mesmas propriedades geométricas e físicas da viga em questão, engastado à fundação e submetido a um carregamento horizontal apenas no último pavimento. Para efetuar a variação de altura do edifício, altera-se a medida do pé-direito (que será sempre $\mathrm{L} / 20)$.

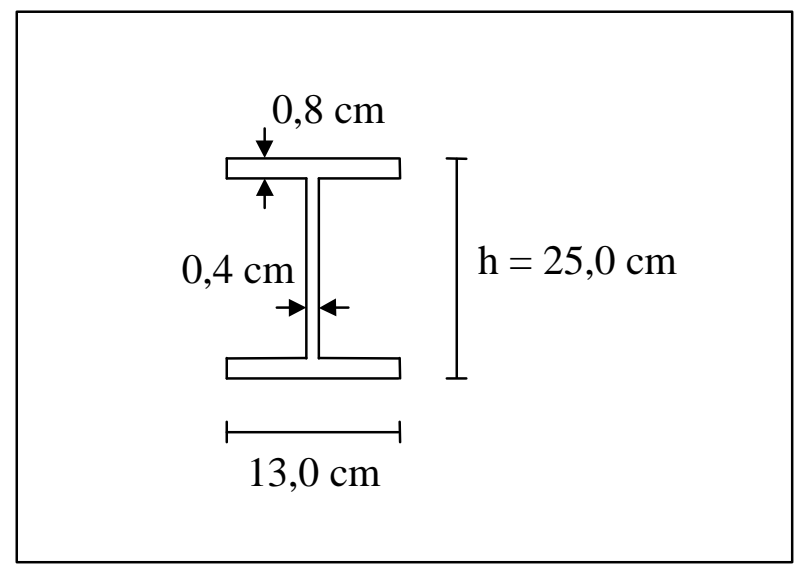

Figura 24 - Seção transversal da viga - exemplo 1

Tabela 2 - Deslocamentos segundo as teorias de Euler-Bernoulli, de Timoshenko, de Alta-ordem e da Elasticidade

\begin{tabular}{|c|c|c|c|c|c|}
\hline \multicolumn{6}{|c|}{ Deslocamento transversal na extremidade livre $(\mathrm{cm})$} \\
\hline \multirow{2}{*}{$\begin{array}{c}\text { Relação } \\
\mathrm{L} / \mathrm{h} \\
\end{array}$} & \multirow{2}{*}{$\begin{array}{c}\text { Teoria de } \\
\text { Euler-Bernoulli }\end{array}$} & \multicolumn{2}{|c|}{ Teoria de Timoshenko } & \multirow{2}{*}{$\begin{array}{c}\text { Teoria de } \\
\text { Alta-ordem }\end{array}$} & \multirow{2}{*}{$\begin{array}{c}\text { Teoria da } \\
\text { Elasticidade }\end{array}$} \\
\hline & & CEASO 01 & PLAIS & & \\
\hline 1 & $-0,00714$ & $-0,0399$ & $-0,0402$ & $-0,0324$ & $-0,0268$ \\
\hline 2 & $-0,0571$ & $-0,123$ & $-0,123$ & $-0,109$ & $-0,0964$ \\
\hline 3 & $-0,193$ & $-0,291$ & $-0,292$ & $-0,270$ & $-0,252$ \\
\hline 4 & $-0,457$ & $-0,588$ & $-0,589$ & $-0,557$ & $-0,536$ \\
\hline 5 & $-0,893$ & $-1,06$ & $-1,06$ & $-1,01$ & $-0,991$ \\
\hline 6 & $-1,54$ & $-1,74$ & $-1,74$ & $-1,68$ & $-1,66$ \\
\hline 8 & $-3,66$ & $-3,92$ & $-3,92$ & $-3,81$ & $-3,81$ \\
\hline 10 & $-7,14$ & $-7,47$ & $-7,47$ & $-7,29$ & $-7,34$ \\
\hline 15 & $-24,1$ & $-24,6$ & $-24,6$ & $-24,1$ & $-24,4$ \\
\hline 20 & $-57,1$ & $-57,8$ & $-57,8$ & $-56,6$ & $-57,5$ \\
\hline
\end{tabular}


Apresentam-se a seguir gráficos de deslocamento transversal pela relação comprimento de viga em relação à altura da seção transversal. Na figura 25 , toma-se o intervalo $1 \leq \mathrm{L} / \mathrm{h} \leq 4$ e na figura 26 , o intervalo $5 \leq \mathrm{L} / \mathrm{h} \leq 20$.

Como se pode ver na figura 25 , os resultados obtidos com o uso da teoria de alta-ordem são os que mais se aproximam dos obtidos com a teoria da elasticidade, vindo a seguir os obtidos com a teoria de Timoshenko e por fim os obtidos com a teoria de Euler-Bernoulli.

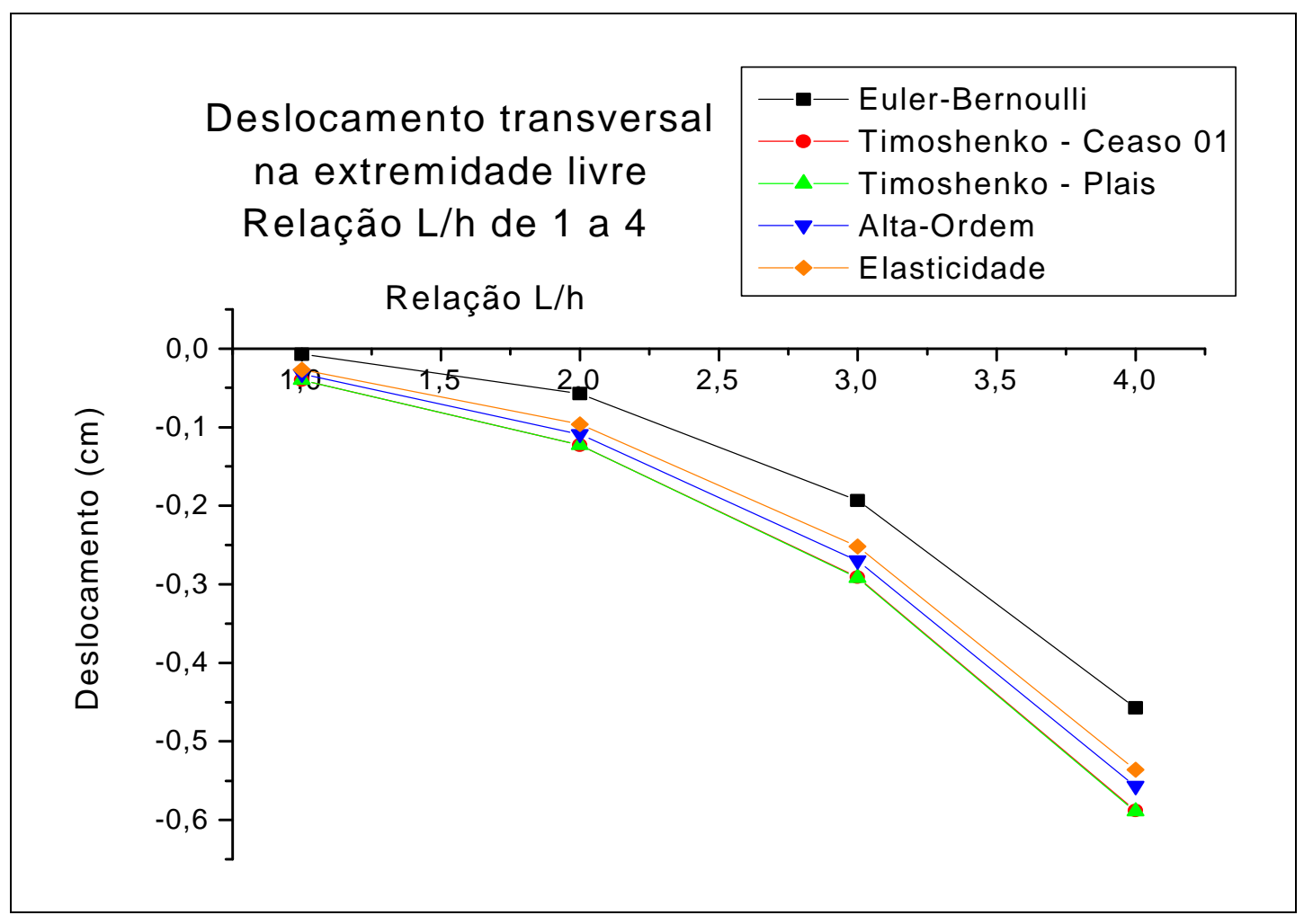

Figura 25 - Deslocamento transversal (Relação L/h de 1 a 4) 


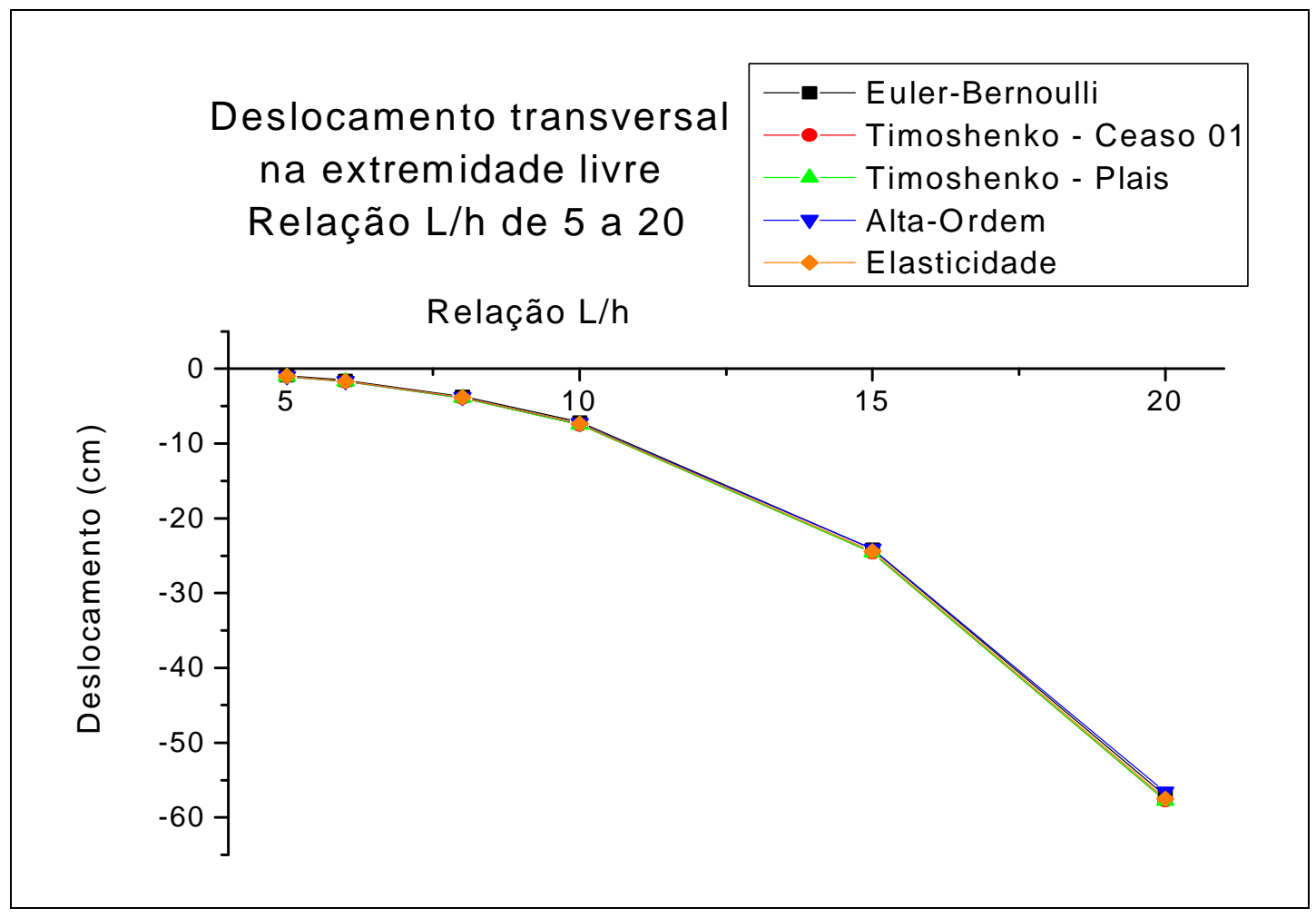

Figura 26 - Deslocamento transversal (Relação L/h de 5 a 20) 


\section{2 - Exemplo 2}

Este exemplo foi extraído de SERRA (1994), sendo originalmente um modelo reduzido analisado e ensaiado por TSO \& BISWAS (1973). Foi feita a conversão de unidades de força e comprimento de libra-força e polegada para newton e centímetro, respectivamente.

Ele consiste numa estrutura de vinte andares, com pé direito de $6,223 \mathrm{~cm}$, formada por dois núcleos resistentes ligados entre si por lintéis, à qual se aplica um momento de torção no topo de $2.306,32$ N.cm. Adota-se para o material componente da estrutura módulo de elasticidade longitudinal $\mathrm{E}=302.591,6 \mathrm{~N} / \mathrm{cm}^{2}$ e transversal $\mathrm{G}=112.592,2 \mathrm{~N} / \mathrm{cm}^{2}$. Os lintéis apresentam seção transversal de largura $0,61976 \mathrm{~cm}$ e altura de $0,9525 \mathrm{~cm}$. Na figura 27 é apresentado um corte da estrutura por um plano horizontal, no nível dos lintéis.

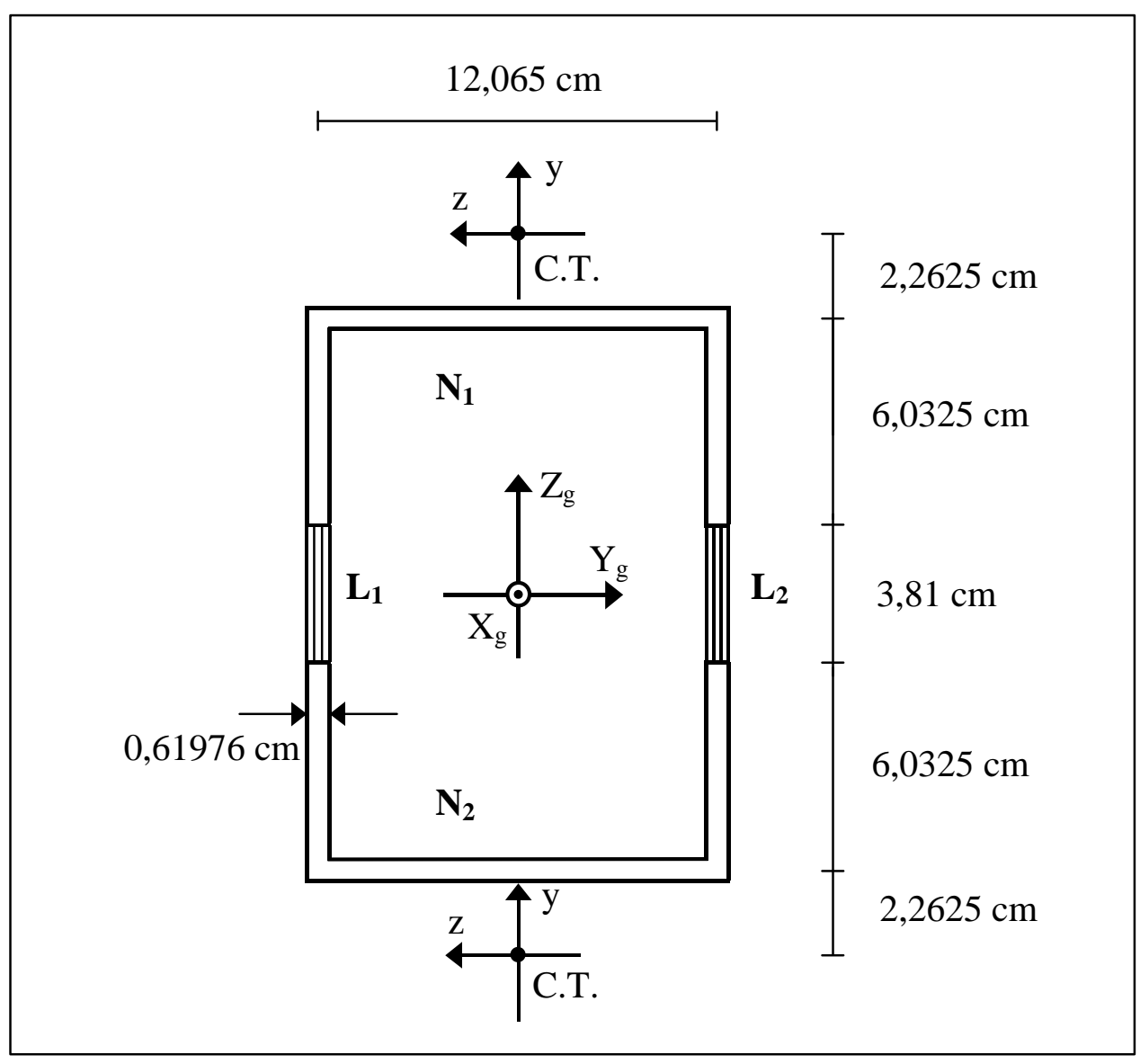

Figura 27 - Corte transversal da estrutura do exemplo 2 
Os fatores de forma da seção de ambos os núcleos são: $\alpha_{\mathrm{yy}}=2,76184$ e $\alpha_{\mathrm{zz}}=2,34787$ quando calculados pelo processo geométrico e $\alpha_{\mathrm{yy}}=3,11191$ e $\alpha_{\mathrm{zz}}=2,36976$, quando calculados pelo processo energético. Os fatores $\alpha_{\mathrm{yz}}$ e $\alpha_{\mathrm{zy}}$ são nulos, devido ao fato de a seção transversal apresentar um eixo de simetria. Neste exemplo, utilizaram-se os fatores de forma obtidos pelo processo de energia.

A posição do centro de torção dos núcleos, a uma distância de $10,200 \mathrm{~cm}$ do nó mestre da laje (sobre o eixo $\mathrm{Z}_{\mathrm{g}}$ ) foi calculada com base na suposição tradicional de que as tensões de cisalhamento sejam constantes ao longo da espessura das paredes. O cálculo baseado na distribuição de tensões obtida com a formulação apresentada neste trabalho leva a que o centro de torção esteja situado a 10,189 cm do nó mestre.

Apresentam-se a seguir tabelas e gráficos que mostram as alterações nos deslocamentos e esforços na estrutura devidas à consideração de deformação por esforço cortante (DPC) nos núcleos. No gráfico das rotações no topo dos pavimentos apresentam-se os resultados obtidos por Serra, cujo trabalho leva em conta a deformação devida ao esforço cortante nas paredes do núcleo, conforme visto no item 2.1 do capítulo 2 . 
Tabela 3 - Rotação em torno do eixo $\mathrm{X}_{\mathrm{g}}$ no topo dos pavimentos

\begin{tabular}{|c|c|c|c|}
\hline Pavimento & $\begin{array}{c}\text { Rotação }\left(10^{-3} \mathrm{rad}\right) \\
\text { sem considerar DPC }\end{array}$ & $\begin{array}{c}\text { Rotação }\left(10^{-3} \mathrm{rad}\right) \\
\text { considerando DPC }\end{array}$ & $\begin{array}{c}\text { Variação } \\
(\%)\end{array}$ \\
\hline 1 & 0,0457 & 0,1008 & $+120,54$ \\
\hline 2 & 0,1696 & 0,2975 & $+75,39$ \\
\hline 3 & 0,3553 & 0,5478 & $+54,21$ \\
\hline 4 & 0,5895 & 0,8409 & $+42,65$ \\
\hline 5 & 0,8619 & 1,168 & $+35,47$ \\
\hline 6 & 1,165 & 1,521 & $+30,62$ \\
\hline 7 & 1,491 & 1,895 & $+27,16$ \\
\hline 8 & 1,835 & 2,286 & $+24,57$ \\
\hline 9 & 2,194 & 2,690 & $+22,59$ \\
\hline 10 & 2,564 & 3,103 & $+21,02$ \\
\hline 11 & 2,942 & 3,523 & $+19,76$ \\
\hline 12 & 3,326 & 3,949 & $+18,74$ \\
\hline 13 & 3,714 & 4,379 & $+17,90$ \\
\hline 14 & 4,105 & 4,811 & $+17,20$ \\
\hline 15 & 4,497 & 5,244 & $+16,61$ \\
\hline 16 & 4,889 & 5,677 & $+16,13$ \\
\hline 17 & 5,278 & 6,108 & $+15,72$ \\
\hline 18 & 5,664 & 6,535 & $+15,38$ \\
\hline 19 & 6,045 & 6,959 & $+15,12$ \\
\hline 20 & 6,418 & 7,363 & $+14,71$ \\
\hline
\end{tabular}

A análise da tabela anterior permite constatar a redução da rigidez à torção da estrutura devida à consideração da deformação por cortante nos núcleos resistentes, o que provoca acréscimo nas rotações em torno do eixo $X_{g}$. Como se sabe, a rigidez de uma estrutura à torção pode ser dividida em duas parcelas: a primeira corresponde à soma das rigidezes à torção dos elementos verticais e a segunda corresponde à soma dos produtos das rigidezes à translação dos elementos verticais pela distância desses elementos ao nó mestre da laje, medida na direção perpendicular à da rigidez considerada. Levar em conta a deformação por cortante faz com que essa segunda parcela se reduza, uma vez que a rigidez à translação nas direções y e z dos núcleos é reduzida e, portanto, a rigidez do edifício à torção se reduz. 
Tabela 4 - Momento torçor em $\mathrm{N}_{1}$.

\begin{tabular}{|c|c|c|c|}
\hline Pavimento & $\begin{array}{c}\text { Momento torçor (N.cm) } \\
\text { sem considerar DPC. }\end{array}$ & $\begin{array}{c}\text { Momento torçor (N.cm) } \\
\text { considerando DPC. }\end{array}$ & $\begin{array}{c}\text { Variação } \\
(\%)\end{array}$ \\
\hline 1 & 84,115 & 441,75 & $+425,17$ \\
\hline 2 & 156,17 & 167,24 & $+7,09$ \\
\hline 3 & 211,32 & 225,47 & $+6,70$ \\
\hline 4 & 255,20 & 266,05 & $+4,25$ \\
\hline 5 & 289,63 & 298,19 & $+2,95$ \\
\hline 6 & 316,72 & 323,51 & $+2,14$ \\
\hline 7 & 337,96 & 343,43 & $+1,62$ \\
\hline 8 & 354,55 & 359,02 & $+1,26$ \\
\hline 9 & 367,43 & 371,16 & $+1,01$ \\
\hline 10 & 377,33 & 380,50 & $+0,84$ \\
\hline 11 & 384,79 & 387,56 & $+0,72$ \\
\hline 12 & 390,25 & 392,73 & $+0,63$ \\
\hline 13 & 393,99 & 396,30 & $+0,58$ \\
\hline 14 & 396,34 & 398,45 & $+0,53$ \\
\hline 15 & 396,95 & 399,31 & $+0,59$ \\
\hline 16 & 397,70 & 398,92 & $+0,31$ \\
\hline 17 & 391,61 & 397,27 & $+1,45$ \\
\hline 18 & 405,13 & 394,32 & $-2,67$ \\
\hline 19 & 336,54 & 386,72 & $-12,98$ \\
\hline 20 & 574,11 & 535,72 & $-6,69$ \\
\hline
\end{tabular}

Obs: Os valores de momento torçor em $\mathrm{N}_{2}$ são idênticos aos de $\mathrm{N}_{1}$. 
Tabela 5 - Esforço cortante no núcleo $\mathrm{N}_{1}$ na direção de seu eixo z local

\begin{tabular}{|c|c|c|c|}
\hline Pavimento & $\begin{array}{c}\text { Esforço cortante (N) } \\
\text { sem considerar DPC }\end{array}$ & $\begin{array}{c}\text { Esforço cortante (N) } \\
\text { considerando DPC }\end{array}$ & $\begin{array}{c}\text { Variação } \\
(\%)\end{array}$ \\
\hline 1 & 104,81 & 69,75 & $-33,45$ \\
\hline 2 & 97,75 & 96,66 & $-1,11$ \\
\hline 3 & 92,34 & 90,95 & $-1,50$ \\
\hline 4 & 88,04 & 86,97 & $-1,21$ \\
\hline 5 & 84,66 & 83,82 & $-0,99$ \\
\hline 6 & 82,01 & 81,34 & $-0,81$ \\
\hline 7 & 79,92 & 79,39 & $-0,67$ \\
\hline 8 & 78,30 & 77,86 & $-0,56$ \\
\hline 9 & 77,03 & 76,67 & $-0,47$ \\
\hline 10 & 76,06 & 75,75 & $-0,41$ \\
\hline 11 & 75,33 & 75,06 & $-0,36$ \\
\hline 12 & 74,80 & 74,55 & $-0,32$ \\
\hline 13 & 74,43 & 74,21 & $-0,30$ \\
\hline 14 & 74,20 & 73,99 & $-0,28$ \\
\hline 15 & 74,14 & 73,91 & $-0,31$ \\
\hline 16 & 74,07 & 73,95 & $-0,16$ \\
\hline 17 & 74,66 & 74,11 & $-0,74$ \\
\hline 18 & 73,34 & 74,40 & $+1,45$ \\
\hline 19 & 80,06 & 75,14 & $-6,15$ \\
\hline 20 & 56,77 & 60,54 & $+6,63$ \\
\hline
\end{tabular}

Obs: $\mathrm{O}$ esforço cortante no núcleo $\mathrm{N}_{2}$ têm os mesmos valores (em módulo) dados na tabela 5, porém em sentido contrário.

$\mathrm{Na}$ estrutura em questão, o momento aplicado no topo do edifício $\left(\mathrm{M}_{\mathrm{t}}=2.306,32 \mathrm{~N} . \mathrm{cm}\right)$ é absorvido pelos momentos torçores nos núcleos $\mathrm{N}_{1}$ e $\mathrm{N}_{2}$ e pelo binário formado pelo esforço cortante na direção do eixo z local de cada núcleo. Como a rigidez à translação em $\mathrm{z}$ de $\mathrm{N}_{1}$ e $\mathrm{N}_{2}$ se reduziu pela adoção da teoria de Timoshenko, o esforço cortante nesses elementos (e o binário por eles formado) também foi reduzido, fazendo com que o momento torçor aumentasse para satisfazer o equilíbrio expresso na eq. (191).

$$
\mathrm{M}_{\mathrm{t}}=\mathrm{M}_{\mathrm{N}_{1}}+\mathrm{M}_{\mathrm{N}_{2}}+\mathrm{R}_{\mathrm{z}} \cdot \mathrm{d}_{\mathrm{CT}}
$$

em que: 
$\mathrm{M}_{\mathrm{t}}$ é o momento total em torno de $\mathrm{X}_{\mathrm{g}}$ a que o pavimento está submetido.

$\mathrm{M}_{\mathrm{N}_{1}}$ é o momento torçor a que o núcleo $\mathrm{N}_{1}$ está submetido.

$\mathrm{M}_{\mathrm{N}_{2}}$ é o momento torçor a que o núcleo $\mathrm{N}_{2}$ está submetido.

$\mathrm{R}_{\mathrm{z}}$ é o esforço cortante na direção $\mathrm{z}$ em cada núcleo.

$\mathrm{d}_{\text {Ст }}$ é a distância entre os centros de torção de $\mathrm{N}_{1}$ e $\mathrm{N}_{2}$.

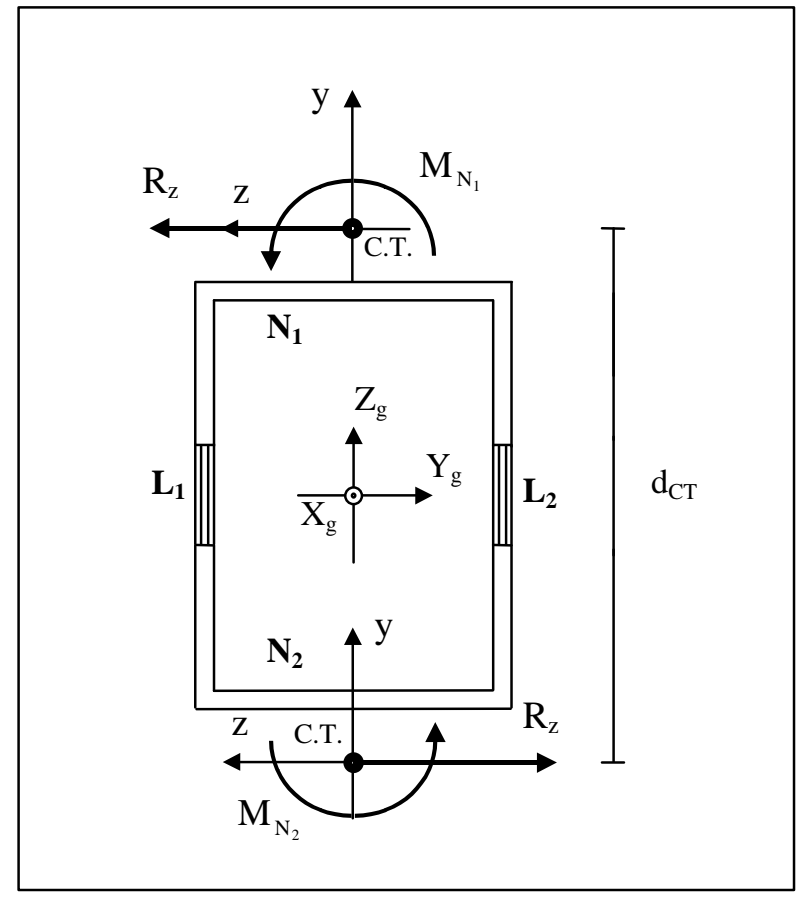

Figura 28 - Equilíbrio à torção do pavimento

Em decorrência do aumento verificado no momento torçor (especialmente no primeiro pavimento) e devido ao fato de que na proximidade do engaste com a fundação o momento torçor é composto apenas de momento de flexo-torção (sendo nulo o momento de torção livre) ocorre um aumento muito expressivo do bimomento na base dos núcleos no primeiro andar, uma vez que o momento de flexo-torção corresponde à derivada do bimomento em relação ao eixo $X_{\mathrm{g}}$. 
Tabela 6 - Bimomento no núcleo $\mathrm{N}_{1}$ (ou no núcleo $\mathrm{N}_{2}$ )

\begin{tabular}{|c|c|c|c|c|}
\hline \multirow{2}{*}{ Pavimento } & \multicolumn{2}{|c|}{$\begin{array}{c}\text { Bimomento }\left(\mathrm{N} . \mathrm{cm}^{2}\right) \\
\text { Bimomento }\left(\mathrm{N} . \mathrm{cm}^{2}\right)\end{array}$} \\
\cline { 2 - 5 } & sem considerar DPC & \multicolumn{2}{c|}{ considerando DPC } \\
\hline & Base & Topo & Base & Topo \\
\hline 1 & $-1.204,0$ & $+690,4$ & $-3.186,6$ & $+459,3$ \\
\hline 2 & $-1.221,8$ & $+276,7$ & $-1.133,7$ & $+135,4$ \\
\hline 3 & $-1.227,4$ & $-47,6$ & $-1.192,0$ & $-157,1$ \\
\hline 4 & $-1.233,5$ & $-304,1$ & $-1.204,6$ & $-387,8$ \\
\hline 5 & $-1.237,4$ & $-506,2$ & $-1.214,7$ & $-570,4$ \\
\hline 6 & $-1.239,9$ & $-665,8$ & $-1.222,2$ & $-714,9$ \\
\hline 7 & $-1.241,1$ & $-791,7$ & $-1.227,3$ & $-829,2$ \\
\hline 8 & $-1.241,1$ & $-891,0$ & $-1.230,5$ & $-919,5$ \\
\hline 9 & $-1.239,9$ & $-969,3$ & $-1.231,8$ & $-991,0$ \\
\hline 10 & $-1.237,3$ & $-1.031,1$ & $-1.231,3$ & $-1.047,4$ \\
\hline 11 & $-1.233,3$ & $-1.079,8$ & $-1.229,1$ & $-1.092,0$ \\
\hline 12 & $-1.227,6$ & $-1.118,1$ & $-1.225,0$ & $-1.127,1$ \\
\hline 13 & $-1.219,9$ & $-1.148,2$ & $-1.218,8$ & $-1.154,7$ \\
\hline 14 & $-1.209,9$ & $-1.172,3$ & $-1.210,1$ & $-1.176,3$ \\
\hline 15 & $-1.196,3$ & $-1.189,4$ & $-1.198,4$ & $-1.193,1$ \\
\hline 16 & $-1.181,0$ & $-1.209,5$ & $-1.183,2$ & $-1.206,0$ \\
\hline 17 & $-1.153,6$ & $-1.199,4$ & $-1.163,5$ & $-1.215,8$ \\
\hline 18 & $-1.148,5$ & $-1.289,4$ & $-1.138,4$ & $-1.223,3$ \\
\hline 19 & $-1.027,9$ & $-984,4$ & $-1.105,9$ & $-1.209,4$ \\
\hline 20 & $-1.311,6$ & $-2.180,6$ & $-1.085,6$ & $-2161,1$ \\
\hline
\end{tabular}

Os valores dados nas quatro tabelas anteriores são agora apresentados graficamente. Na figura 29 pode-se verificar que os resultados obtidos com o programa CEASO 01 com a consideração de deformação por cortante estão mais próximos dos obtidos por Serra do que os obtidos por CEASO 01 sem levar em conta a distorção no núcleo. 


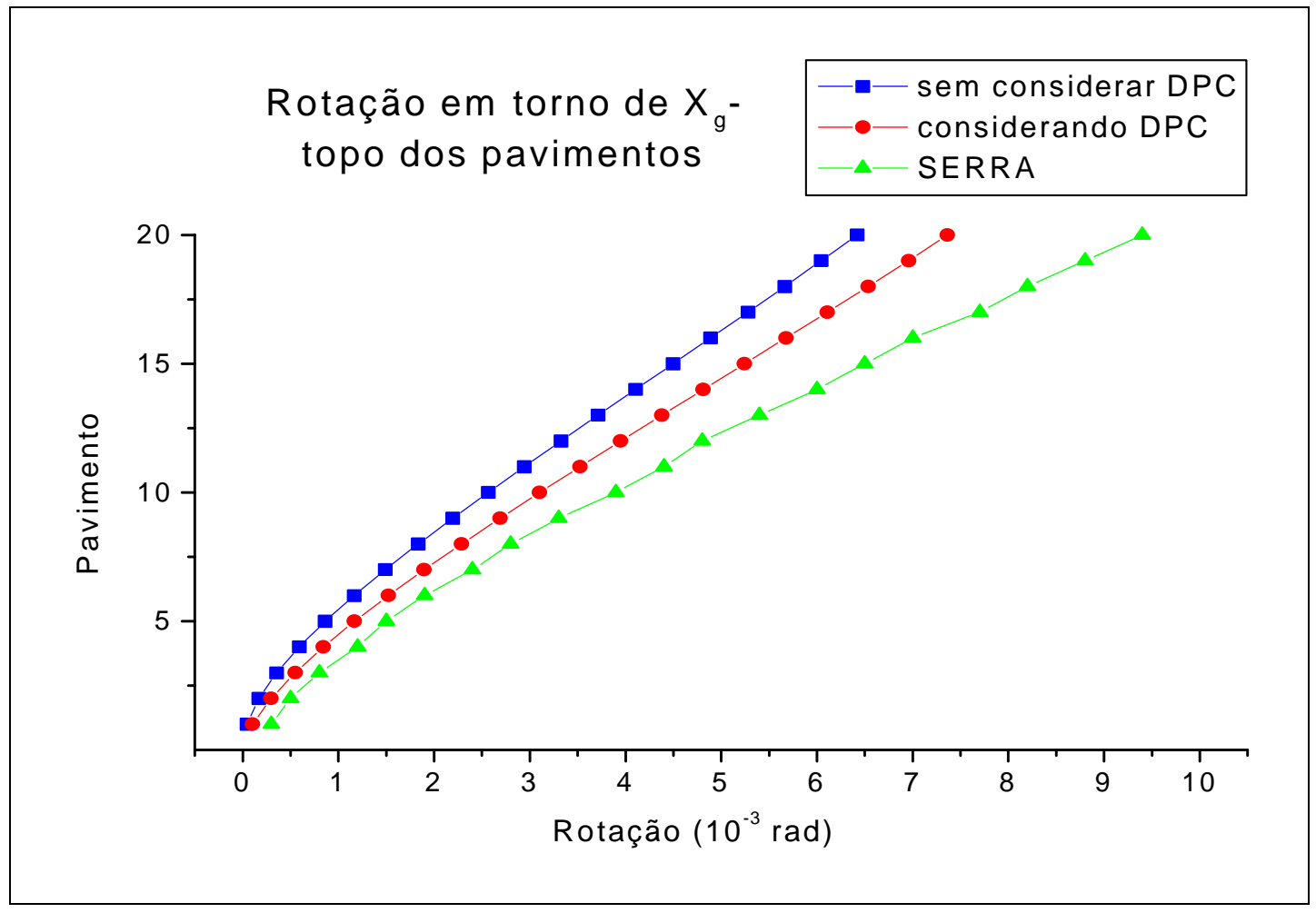

Figura 29 - Rotação em torno de $\mathrm{X}_{\mathrm{g}}$ no topo dos pavimentos

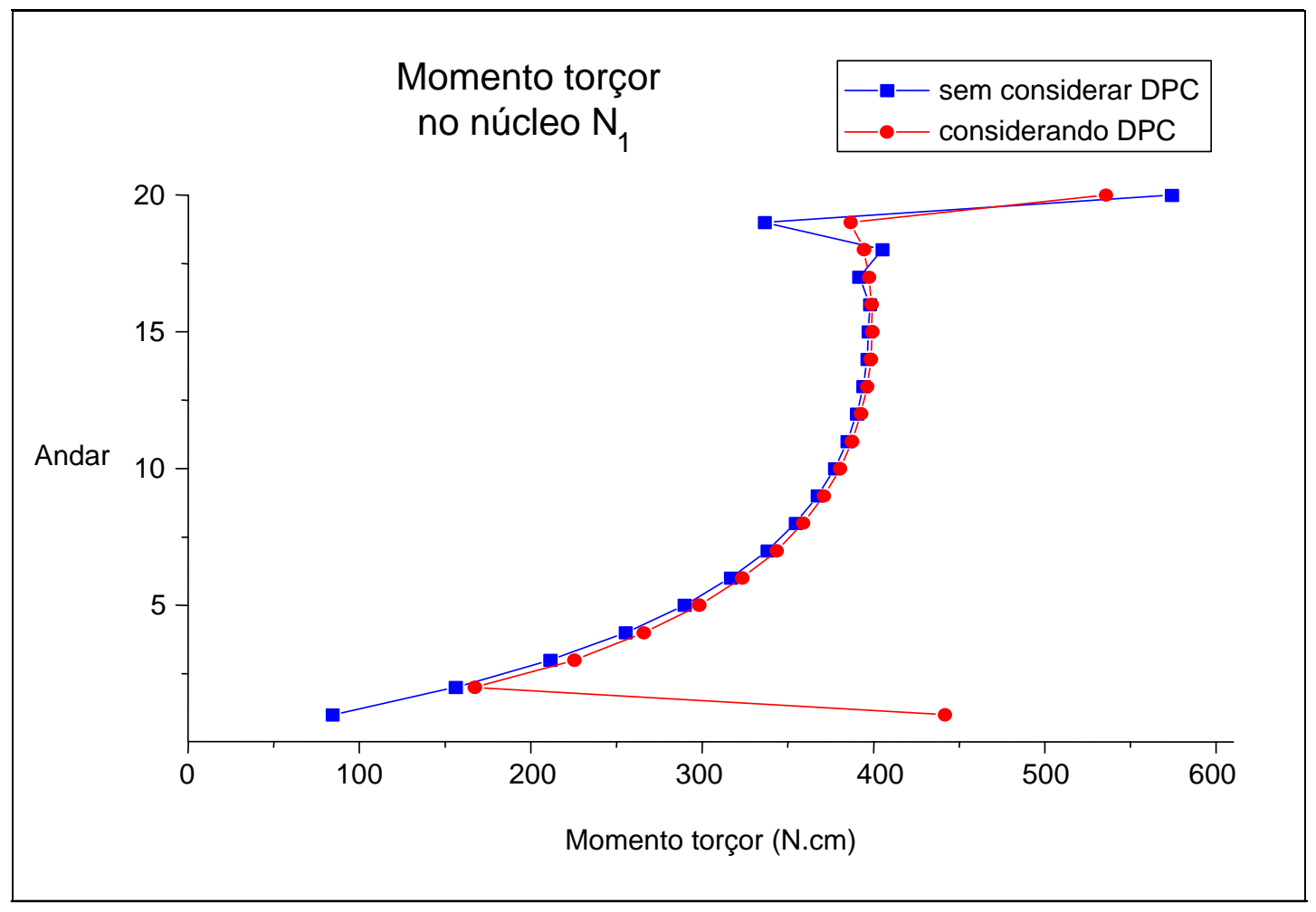

Figura 30 - Momento torçor no núcleo $\mathrm{N}_{1}$ 


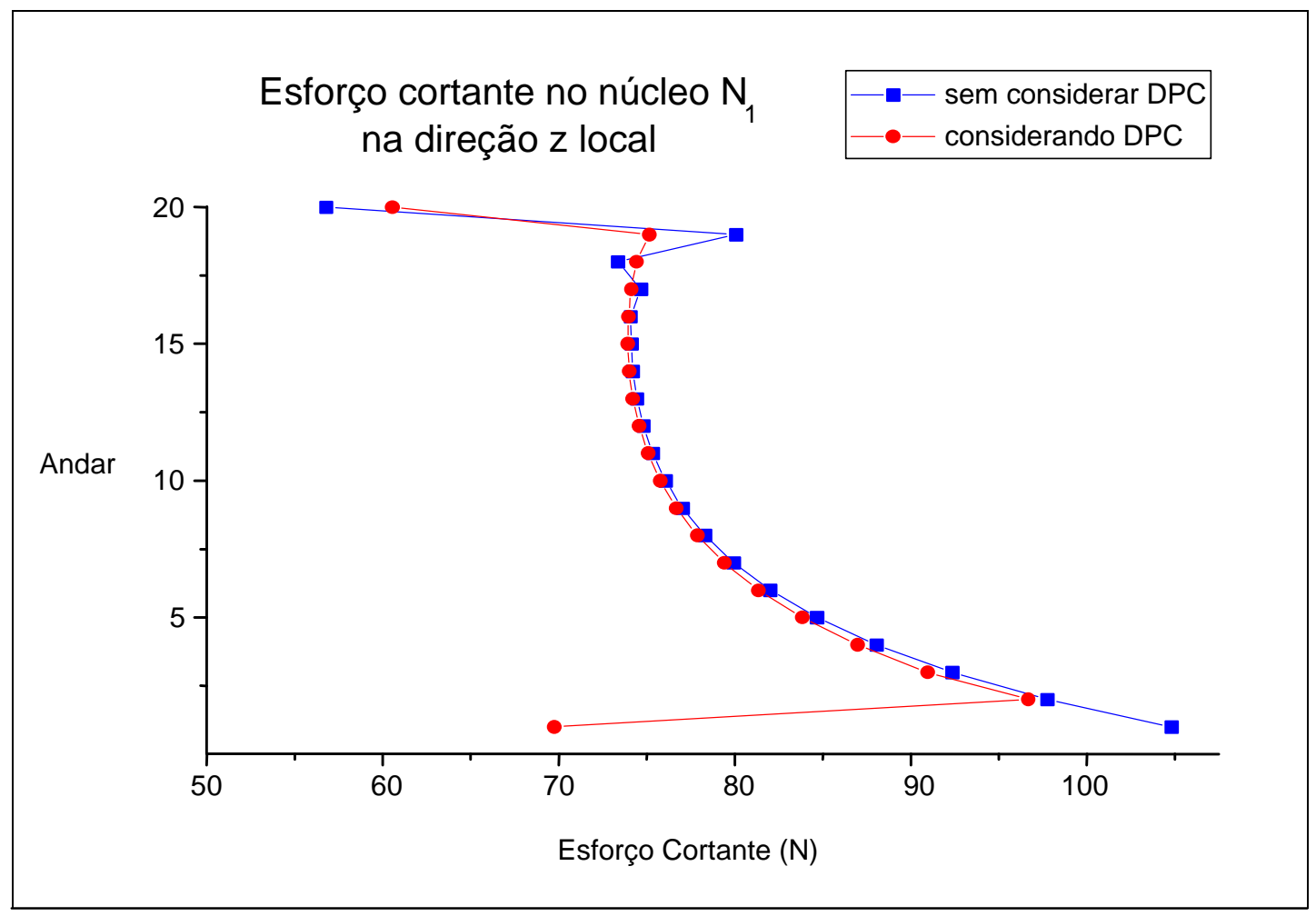

Figura 31 - Esforço cortante na direção do eixo z local do núcleo $\mathrm{N}_{1}$

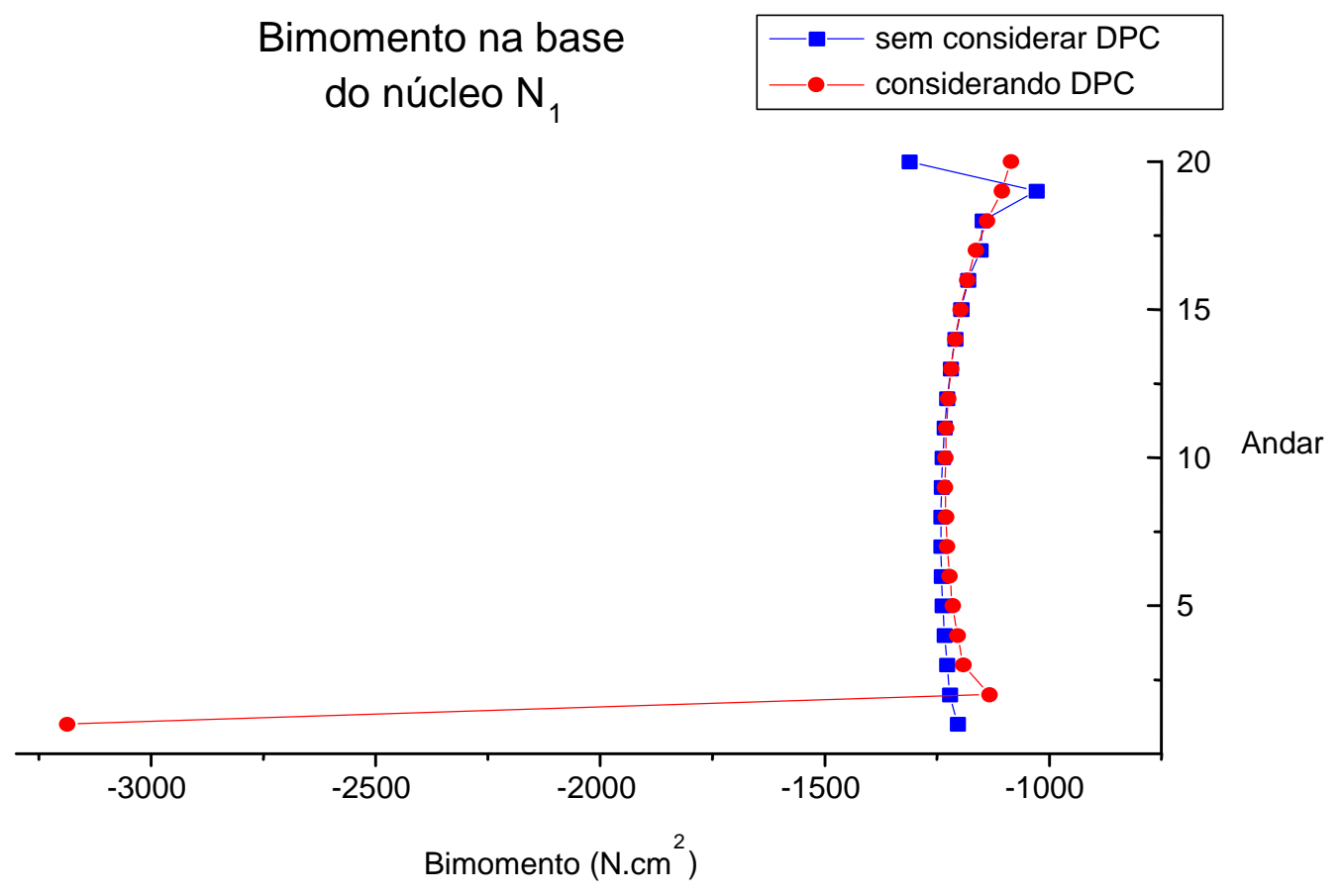

Figura 32 - Bimomento na base do núcleo $\mathrm{N}_{1}$ 


\section{3 - Exemplo 3}

Este exemplo foi desenvolvido por SILVA (1989) e analisado também por MORI (1992) e MATIAS JR. (1997).

A estrutura analisada é um edifício de 15 pavimentos e pé-direito de 4,0 m, cujo sistema de contraventamento é constituído por quatro pórticos planos, quatro pilares de flexão em torno de dois planos e um núcleo estrutural de seção transversal em forma de $\mathrm{U}$ e parede de espessura constante de $15 \mathrm{~cm}$.

$\mathrm{Na}$ figura 33 encontra-se a planta do pavimento-tipo do edifício. Este apresenta vigas de seção retangular $20 \mathrm{~cm}$ x $60 \mathrm{~cm}$ e pilares de seção também retangular de $25 \mathrm{~cm} \times 50 \mathrm{~cm}$. Todos os elementos da estrutura apresentam módulo de elasticidade longitudinal $\mathrm{E}=2.000 \mathrm{kN} / \mathrm{cm}^{2}$ e transversal $\mathrm{G}=800 \mathrm{kN} / \mathrm{cm}^{2}$.

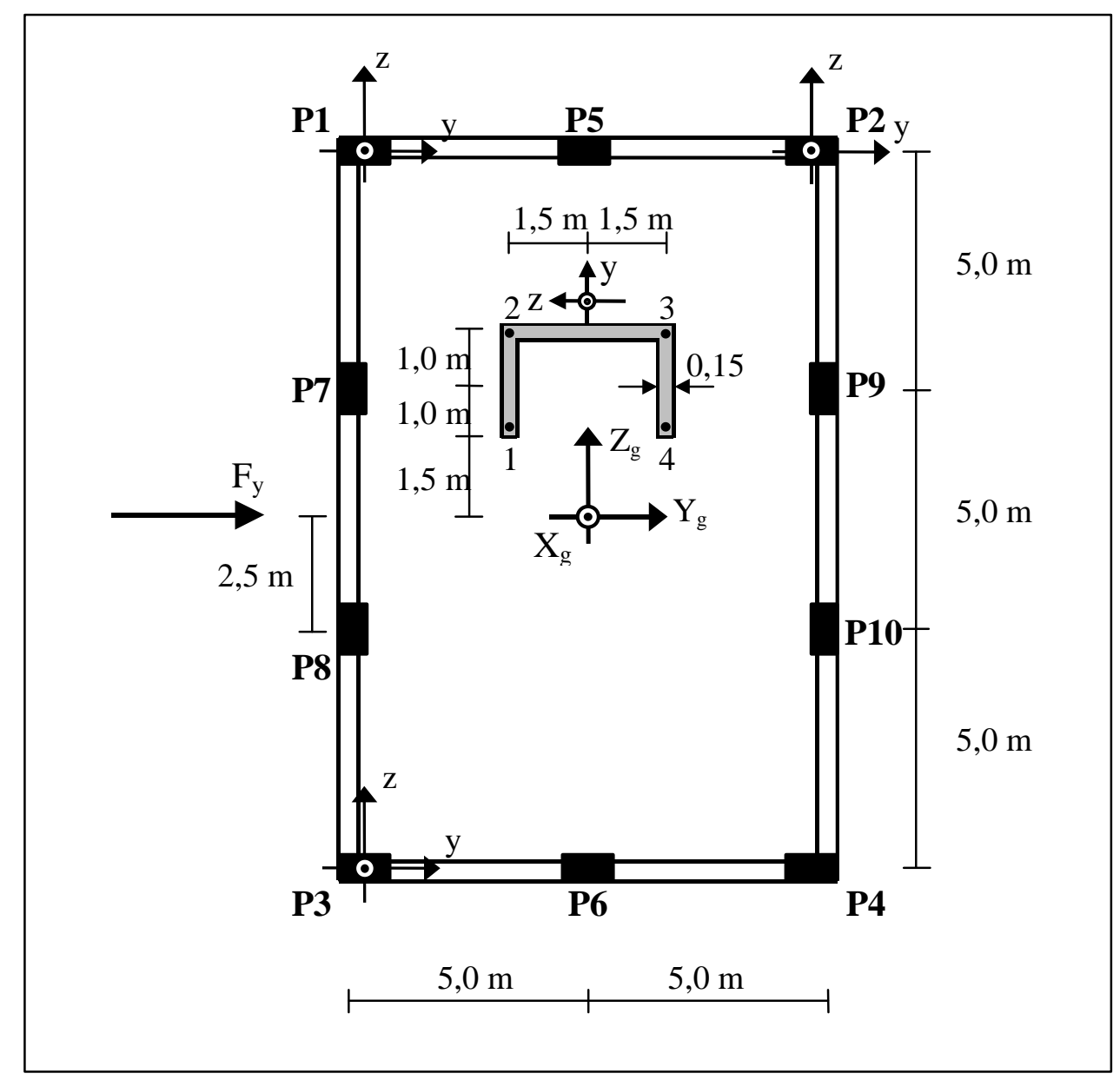

Figura 33 - Planta do pavimento-tipo do exemplo 3 
Os fatores de forma da seção do núcleo são: $\alpha_{\mathrm{yy}}=2,36791$ e $\alpha_{\mathrm{zz}}=2,98366$ quando calculados pelo processo geométrico e $\alpha_{\mathrm{yy}}=2,51300$ e $\alpha_{\mathrm{zz}}=3,00295$, quando calculados pelo processo energético, tendo sido estes últimos os valores utilizados na análise deste exemplo

O carregamento aplicado consiste em forças horizontais e verticais. No último pavimento, as forças aplicadas são:

- Carregamento uniformemente distribuído sobre as vigas de $10 \mathrm{kN} / \mathrm{m}$.

- Forças verticais aplicadas nos pontos 1, 2, 3 e 4 do núcleo de 35 kN, $90 \mathrm{kN}, 90 \mathrm{kN}$ e $35 \mathrm{kN}$, respectivamente.

- Força horizontal aplicada na direção e sentido do eixo $Y_{g}$ de 25,5 kN .

Em cada um dos demais pavimentos, as forças aplicadas são:

- Carregamento uniformemente distribuído sobre as vigas de $20 \mathrm{kN} / \mathrm{m}$.

- Forças verticais aplicadas nos pontos 1, 2, 3 e 4 do núcleo de $70 \mathrm{kN}$, $180 \mathrm{kN}, 180 \mathrm{kN}$ e $70 \mathrm{kN}$, respectivamente.

- Força horizontal aplicada na direção e sentido do eixo $Y_{g}$ de 51 kN.

Apresentam-se a seguir tabelas comparativas de deslocamentos e esforços, com e sem consideração da deformação pelo esforço cortante, tanto no núcleo como nos pilares. Apresentam-se também resultados obtidos por Silva, que utilizou o modelo de substituição das paredes do núcleo por pilares de rigidez equivalente cuja matriz de rigidez leva em conta o efeito da distorção devida ao esforço cortante, conforme visto no capítulo 2 . 
Tabela 7 - Translação do nó mestre das lajes na direção de $Y_{g}$

\begin{tabular}{|c|c|c|c|}
\hline Laje & $\begin{array}{c}\text { Translação }(\mathrm{cm}) \\
\text { sem considerar DPC }\end{array}$ & $\begin{array}{c}\text { Translação (cm) } \\
\text { considerando DPC }\end{array}$ & $\begin{array}{c}\text { Translação (cm) } \\
\text { Silva }\end{array}$ \\
\hline 1 & 0,51 & 0,58 & 0,59 \\
\hline 2 & 1,49 & 1,62 & 1,64 \\
\hline 3 & 2,68 & 2,86 & 2,90 \\
\hline 4 & 4,00 & 4,23 & 4,27 \\
\hline 5 & 5,41 & 5,67 & 5,72 \\
\hline 6 & 6,85 & 7,14 & 7,19 \\
\hline 7 & 8,30 & 8,62 & 8,65 \\
\hline 8 & 9,73 & 10,07 & 10,09 \\
\hline 9 & 11,12 & 11,47 & 11,49 \\
\hline 10 & 12,44 & 12,82 & 12,81 \\
\hline 11 & 13,70 & 14,09 & 14,07 \\
\hline 12 & 14,89 & 15,29 & 15,25 \\
\hline 13 & 16,01 & 16,41 & 16,36 \\
\hline 14 & 17,05 & 17,46 & 17,39 \\
\hline 15 & 18,05 & 18,45 & 18,36 \\
\hline
\end{tabular}

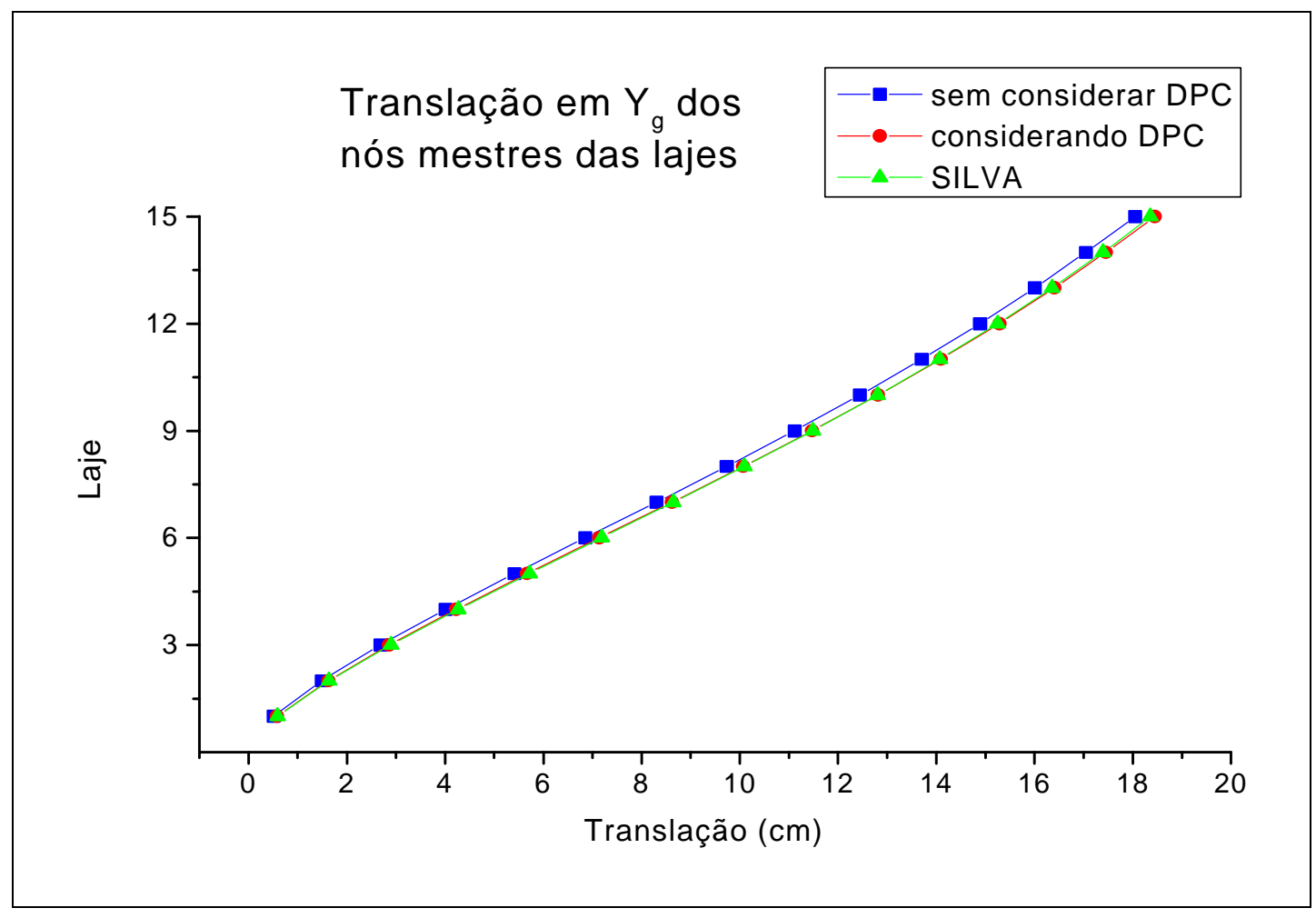

Figura 34 - Translação em $\mathrm{Y}_{\mathrm{g}}$ do nó mestre das lajes 
Tabela 8 - Rotação em torno do eixo vertical $X_{g}$

\begin{tabular}{|c|c|c|c|}
\hline Laje & $\begin{array}{c}\text { Rotação }\left(10^{-3} \text { rad }\right) \\
\text { sem considerar DPC }\end{array}$ & $\begin{array}{c}\text { Rotação }\left(10^{-3} \mathrm{rad}\right) \\
\text { considerando DPC }\end{array}$ & $\begin{array}{c}\text { Rotação }\left(10^{-3} \mathrm{rad}\right) \\
\text { Silva }\end{array}$ \\
\hline 1 & 0,6912 & 0,6817 & 0,65 \\
\hline 2 & 1,631 & 1,620 & 1,54 \\
\hline 3 & 2,450 & 2,440 & 2,33 \\
\hline 4 & 3,130 & 3,125 & 2,99 \\
\hline 5 & 3,688 & 3,688 & 3,54 \\
\hline 6 & 4,139 & 4,145 & 3,98 \\
\hline 7 & 4,498 & 4,509 & 4,33 \\
\hline 8 & 4,775 & 4,791 & 4,61 \\
\hline 9 & 4,976 & 4,997 & 4,81 \\
\hline 10 & 5,108 & 5,133 & 4,94 \\
\hline 11 & 5,173 & 5,201 & 5,01 \\
\hline 12 & 5,171 & 5,202 & 5,01 \\
\hline 13 & 5,102 & 5,134 & 4,95 \\
\hline 14 & 4,962 & 4,995 & 4,81 \\
\hline 15 & 4,753 & 4,790 & 4,62 \\
\hline
\end{tabular}

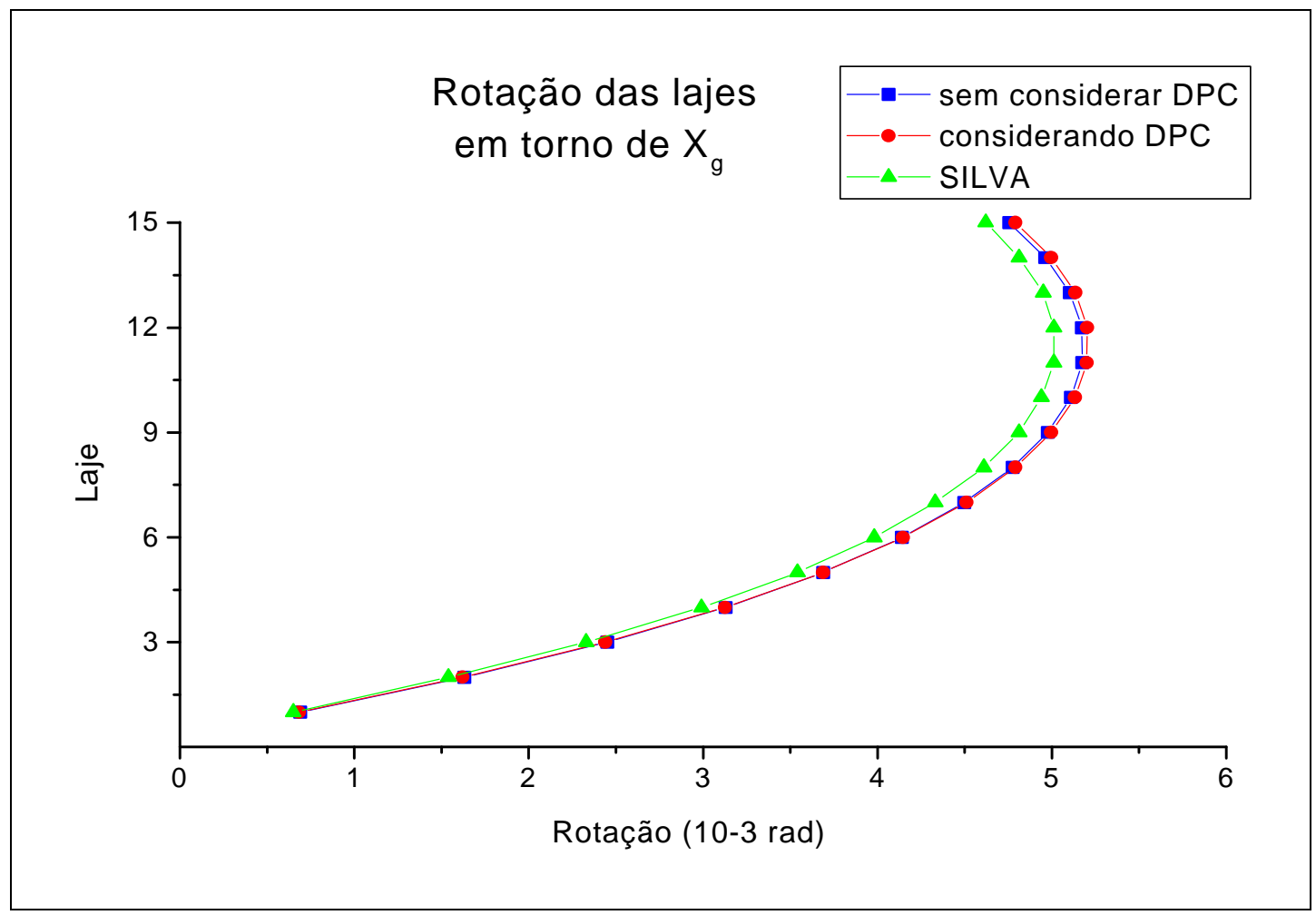

Figura 35 - Rotação das lajes em torno de $X_{g}$ 
Tabela 9 - Momento fletor em torno do eixo $\mathrm{z}$ local do pilar 3 na extremidade inferior

\begin{tabular}{|c|c|c|c|}
\hline Pavimento & $\begin{array}{c}\mathrm{M}_{\mathrm{z}}(\mathrm{kN} . \mathrm{m}) \\
\text { sem considerar DPC }\end{array}$ & $\begin{array}{c}\mathrm{M}_{\mathrm{z}}(\mathrm{kN} . \mathrm{m}) \\
\text { considerando DPC }\end{array}$ & $\begin{array}{c}\mathrm{M}_{\mathrm{z}}(\mathrm{kN} . \mathrm{m}) \\
\text { Silva }\end{array}$ \\
\hline 1 & 133,78 & 139,87 & 135,8 \\
\hline 2 & 113,39 & 114,38 & 96,1 \\
\hline 3 & 107,58 & 108,83 & 94,1 \\
\hline 4 & 104,44 & 105,20 & 88,7 \\
\hline 5 & 99,51 & 100,05 & 83,0 \\
\hline 6 & 93,54 & 93,90 & 76,2 \\
\hline 7 & 86,59 & 86,82 & 68,7 \\
\hline 8 & 78,94 & 79,08 & 60,6 \\
\hline 9 & 70,79 & 70,85 & 52,0 \\
\hline 10 & 62,33 & 62,34 & 43,2 \\
\hline 11 & 53,74 & 53,69 & 34,4 \\
\hline 12 & 45,17 & 45,06 & 25,5 \\
\hline 13 & 36,92 & 36,70 & 17,0 \\
\hline 14 & 28,53 & 28,34 & 8,4 \\
\hline 15 & 24,86 & 24,02 & 4,5 \\
\hline
\end{tabular}

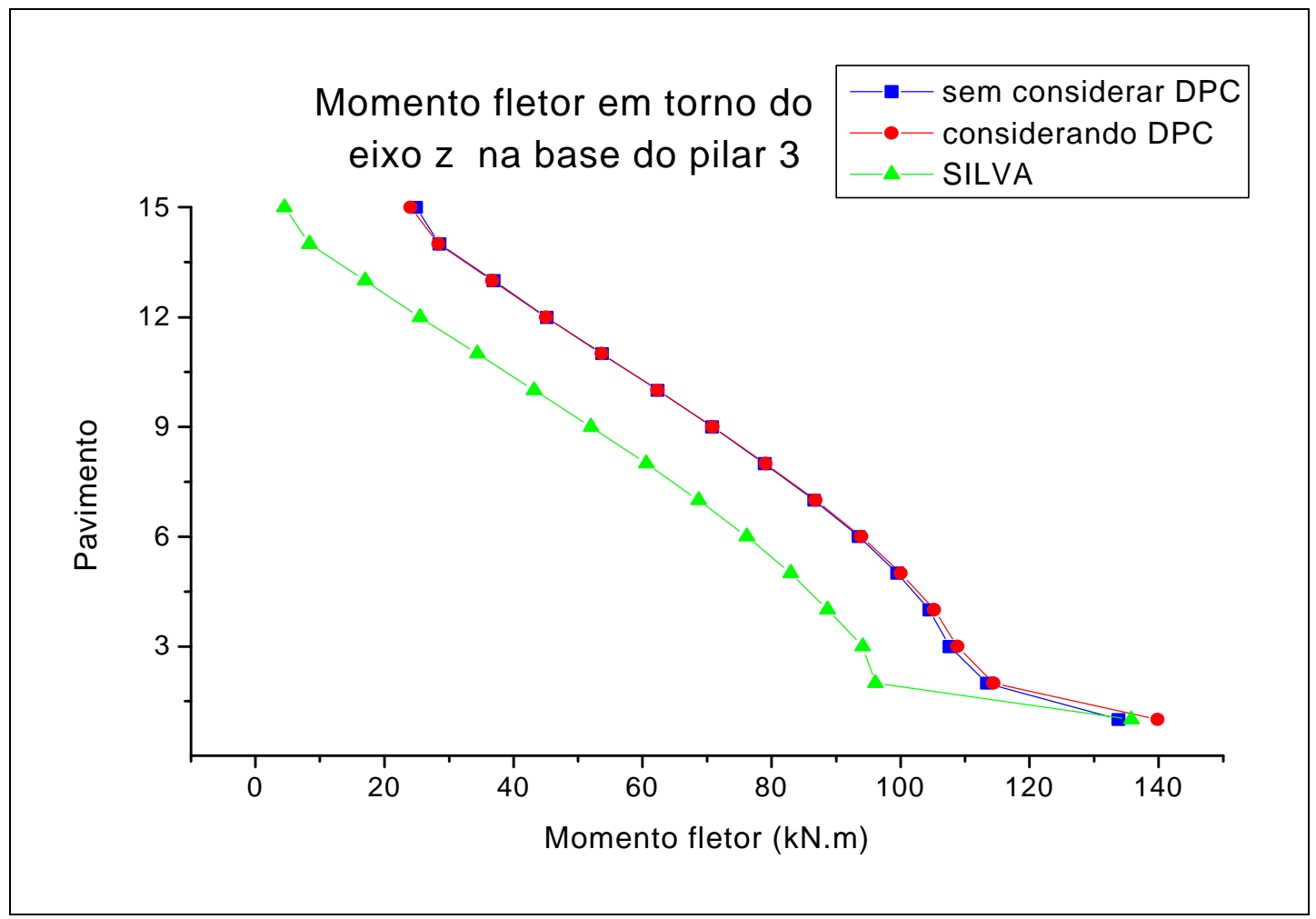

Figura 36 - Momento fletor em torno do eixo z na base do pilar 3 
Tabela 10 - Momento fletor em torno do eixo $\mathrm{z}$ local do pilar 2, em sua extremidade inferior

\begin{tabular}{|c|c|c|}
\hline Pavimento & $\begin{array}{c}\mathrm{M}_{\mathrm{z}}(\mathrm{kN} . \mathrm{m}) \\
\text { sem considerar DPC }\end{array}$ & $\begin{array}{c}\mathrm{M}_{\mathrm{z}}(\mathrm{kN} . \mathrm{m}) \\
\text { considerando DPC }\end{array}$ \\
\hline 1 & $+7,27$ & $-4,82$ \\
\hline 2 & $-19,94$ & $-21,80$ \\
\hline 3 & $-36,00$ & $-38,23$ \\
\hline 4 & $-46,30$ & $-47,59$ \\
\hline 5 & $-52,96$ & $-53,77$ \\
\hline 6 & $-56,66$ & $-57,11$ \\
\hline 7 & $-58,14$ & $-58,34$ \\
\hline 8 & $-57,92$ & $-57,97$ \\
\hline 9 & $-56,52$ & $-56,45$ \\
\hline 10 & $-54,37$ & $-54,21$ \\
\hline 11 & $-51,89$ & $-51,64$ \\
\hline 12 & $-49,42$ & $-49,08$ \\
\hline 13 & $-47,58$ & $-47,05$ \\
\hline 14 & $-45,37$ & $-44,91$ \\
\hline 15 & $-52,59$ & $-50,71$ \\
\hline
\end{tabular}

Tabela 11 - Momento fletor em torno do eixo y local do núcleo, na extremidade inferior

\begin{tabular}{|c|c|c|c|}
\hline Pavimento & $\begin{array}{c}\mathrm{M}_{\mathrm{z}}(\mathrm{kN} . \mathrm{m}) \\
\text { sem considerar DPC }\end{array}$ & $\begin{array}{c}\mathrm{M}_{\mathrm{z}}(\mathrm{kN} . \mathrm{m}) \\
\text { considerando DPC }\end{array}$ & $\begin{array}{c}\mathrm{M}_{\mathrm{z}}(\mathrm{kN} . \mathrm{m}) \\
\text { Silva }\end{array}$ \\
\hline 1 & $-9.804,96$ & $-9.663,59$ & $-9.494,3$ \\
\hline 2 & $-7.462,12$ & $-7.418,14$ & $-7.280,6$ \\
\hline 3 & $-5.590,08$ & $-5.577,32$ & $-5.472,6$ \\
\hline 4 & $-4.044,12$ & $-4.056,94$ & $-3.987,1$ \\
\hline 5 & $-2.777,80$ & $-2.806,09$ & $-2.763,5$ \\
\hline 6 & $-1.745,47$ & $-1.783,23$ & $-1.762,5$ \\
\hline 7 & $-914,09$ & $-956,72$ & $-952,7$ \\
\hline 8 & $-257,10$ & $-301,54$ & $-310,1$ \\
\hline 9 & $+245,56$ & $+201,37$ & $+183,6$ \\
\hline 10 & $+608,48$ & $+565,96$ & $+542,3$ \\
\hline 11 & $+841,45$ & $+801,63$ & $+774,9$ \\
\hline 12 & $+949,71$ & $+913,49$ & $+886,4$ \\
\hline 13 & $+934,71$ & $+902,94$ & $+887,9$ \\
\hline 14 & $+790,90$ & $+765,52$ & $+745,4$ \\
\hline 15 & $+527,87$ & $+507,44$ & $+496,0$ \\
\hline
\end{tabular}




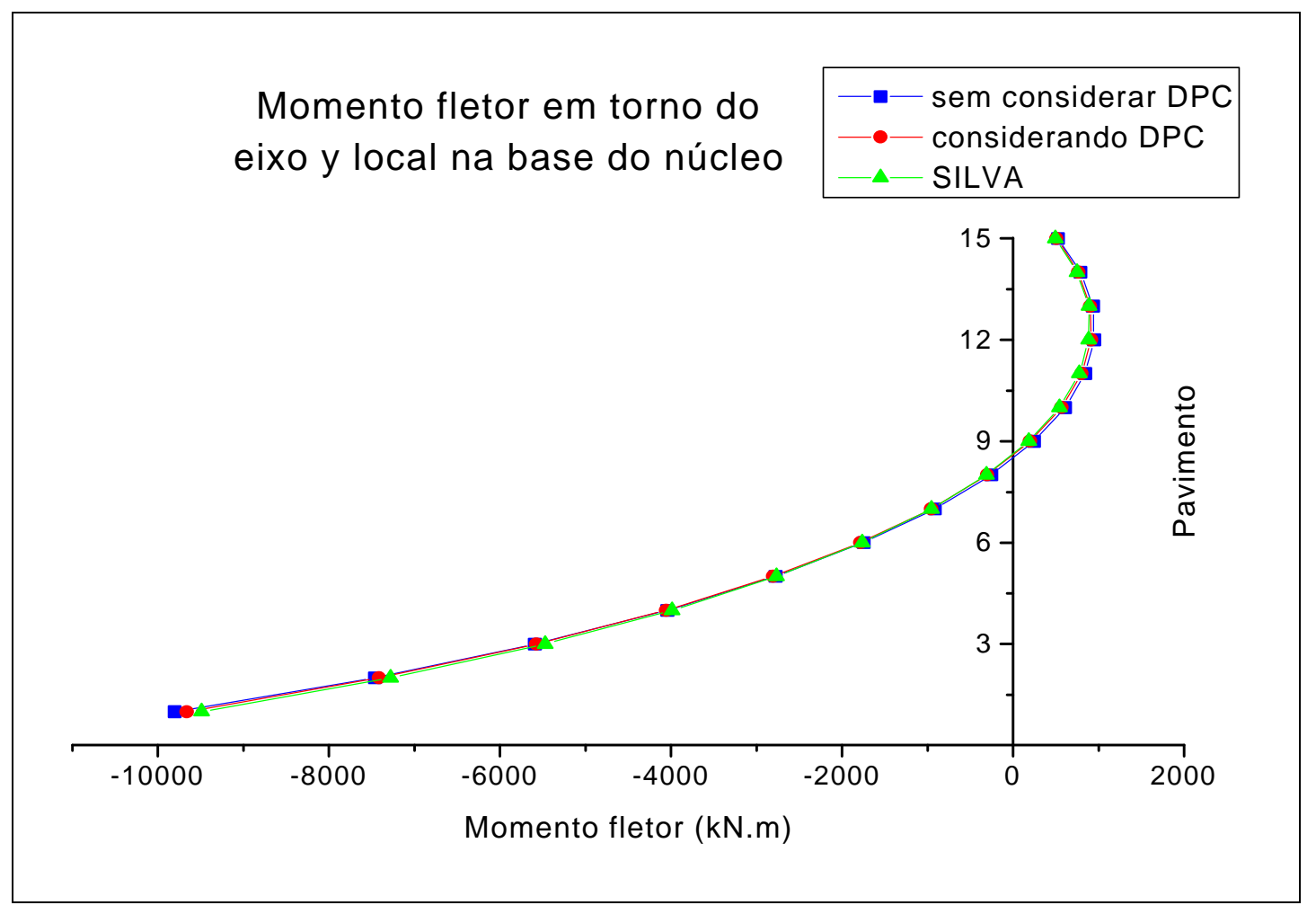

Figura 37 - Momento fletor em torno do eixo y local na base do núcleo

Tabela 12 - Esforço cortante na direção do eixo z local do núcleo

\begin{tabular}{|c|c|c|c|c|c|}
\hline \multirow[b]{2}{*}{ Andar } & \multicolumn{2}{|c|}{ Sem considerar DPC } & \multicolumn{2}{|c|}{ Considerando DPC } & \multirow{2}{*}{$\begin{array}{c}\text { Silva } \\
\text { Cortante } \\
(\mathrm{kN})\end{array}$} \\
\hline & $\begin{array}{c}\text { Cortante } \\
(\mathrm{kN})\end{array}$ & $\begin{array}{l}\% \text { esforço cor- } \\
\text { tante no andar }\end{array}$ & $\begin{array}{c}\text { Cortante } \\
(\mathrm{kN})\end{array}$ & $\begin{array}{l}\% \text { esforço cor- } \\
\text { tante no andar }\end{array}$ & \\
\hline 1 & 585,71 & 79,20 & 561,36 & 75,91 & 553,4 \\
\hline 2 & 468,01 & 67,98 & 460,21 & 66,84 & 452,0 \\
\hline 3 & 386,49 & 60,63 & 380,09 & 59,62 & 371,4 \\
\hline 4 & 316,60 & 53,98 & 312,71 & 53,32 & 305,9 \\
\hline 5 & 258,06 & 48,19 & 255,72 & 47,75 & 250,3 \\
\hline 6 & 207,84 & 42,90 & 206,63 & 42,65 & 202,4 \\
\hline 7 & 164,24 & 37,89 & 163,79 & 37,78 & 160,6 \\
\hline 8 & 125,66 & 32,85 & 125,73 & 32,87 & 123,5 \\
\hline 9 & 90,73 & 27,37 & 91,15 & 27,50 & 89,7 \\
\hline 10 & 58,24 & 20,76 & 58,92 & 21,01 & 58,1 \\
\hline 11 & 27,07 & 11,80 & 27,97 & 12,19 & 27,9 \\
\hline 12 & $-3,75$ & - & $-2,64$ & - & $-2,1$ \\
\hline 13 & $-35,95$ & - & $-34,35$ & - & $-33,1$ \\
\hline 14 & $-65,76$ & - & $-64,52$ & - & $-62,4$ \\
\hline 15 & $-131,97$ & - & $-126,86$ & - & $-124,0$ \\
\hline
\end{tabular}




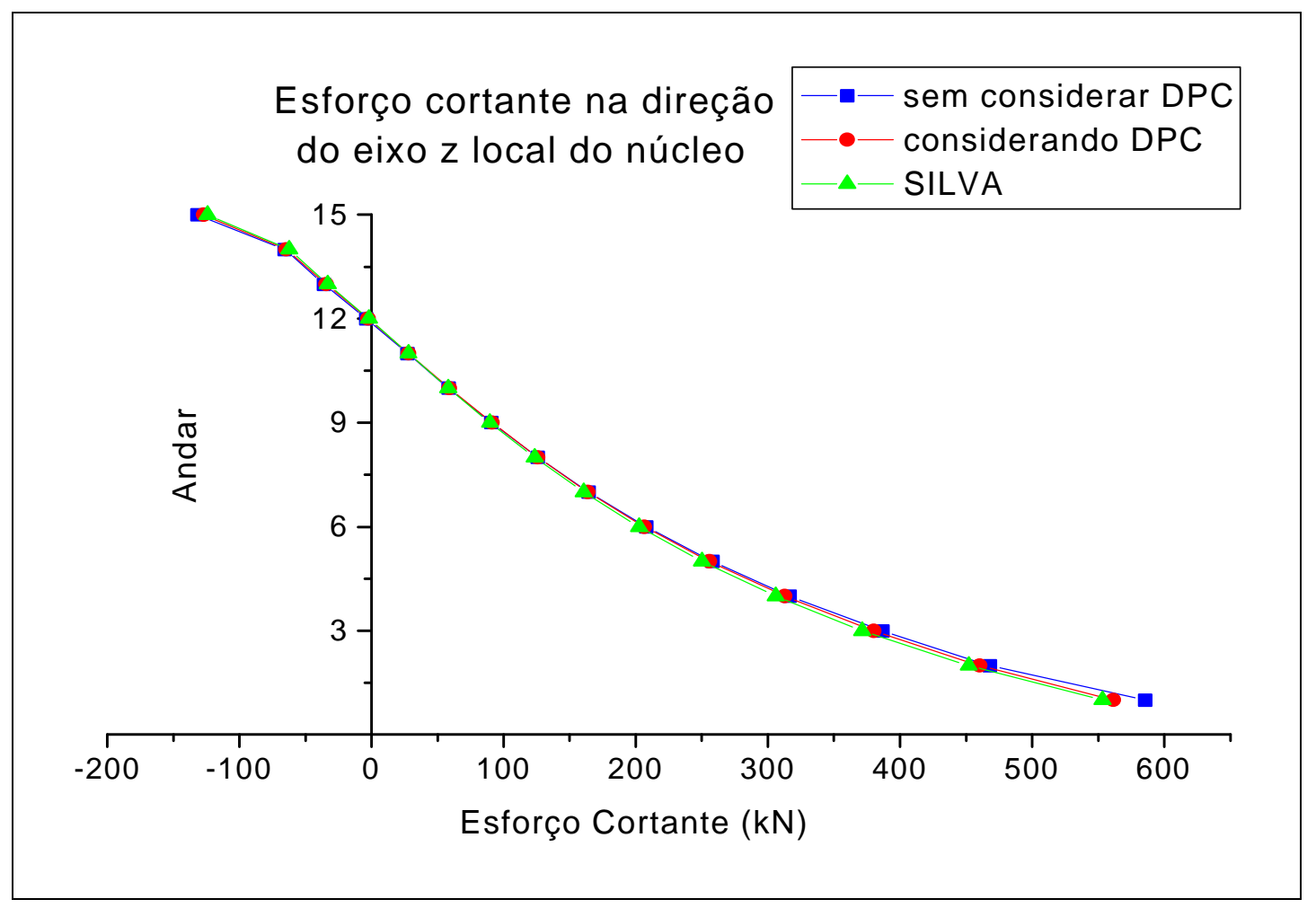

Figura 38 - Esforço cortante na direção do eixo z local do núcleo

Tabela 13 - Somatória de esforços cortantes nos pilares na direção $\mathrm{Y}_{\mathrm{g}}$

\begin{tabular}{|c|c|c|c|}
\hline Andar & $\begin{array}{c}\sum \text { cortantes (kN) } \\
\text { sem considerar DPC. }\end{array}$ & $\begin{array}{c}\sum \text { cortantes }(\mathrm{kN}) \\
\text { considerando DPC. }\end{array}$ & $\begin{array}{c}\text { Variação } \\
(\%)\end{array}$ \\
\hline 1 & 153,79 & 178,14 & $+15,83$ \\
\hline 2 & 220,49 & 228,29 & $+3,54$ \\
\hline 3 & 251,01 & 257,41 & $+2,55$ \\
\hline 4 & 269,90 & 273,79 & $+1,44$ \\
\hline 5 & 277,44 & 279,78 & $+0,84$ \\
\hline 6 & 276,66 & 277,87 & $+0,44$ \\
\hline 7 & 269,26 & 269,71 & $+0,17$ \\
\hline 8 & 256,84 & 256,77 & $-0,03$ \\
\hline 9 & 240,77 & 240,35 & $-0,17$ \\
\hline 10 & 222,26 & 221,58 & $-0,31$ \\
\hline 11 & 202,43 & 201,53 & $-0,44$ \\
\hline 12 & 182,50 & 181,14 & $-0,74$ \\
\hline 13 & 163,45 & 161,85 & $-0,98$ \\
\hline 14 & 142,26 & 141,02 & $-0,87$ \\
\hline 15 & 157,47 & 152,36 & $-3,25$ \\
\hline
\end{tabular}




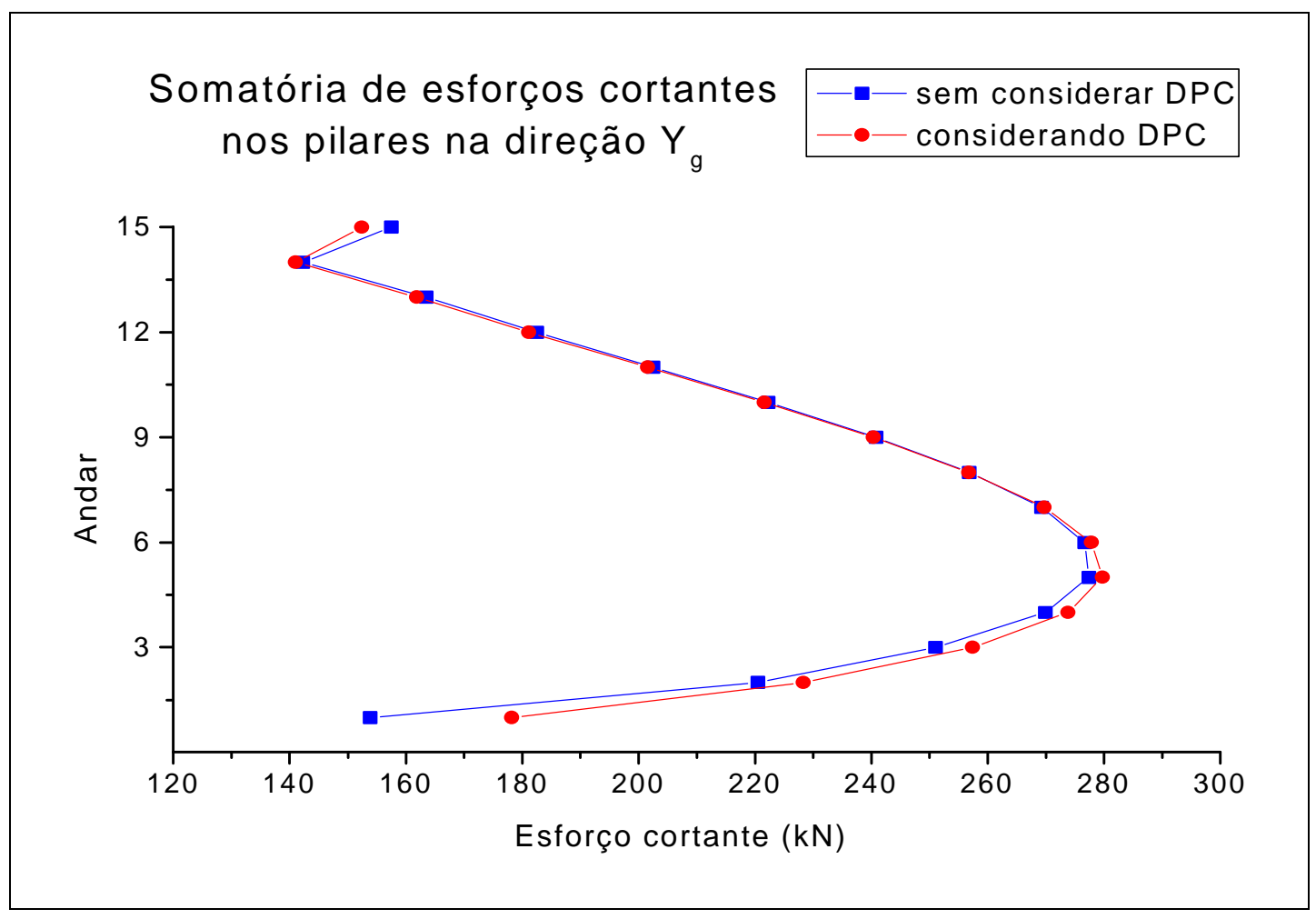

Figura 39 - Somatória de esforços cortantes nos pilares na direção $Y_{g}$

Tabela 14 - Esforço cortante no pilar 1 na direção y local

\begin{tabular}{|c|c|c|c|}
\hline Andar & $\begin{array}{c}\text { Cortante }(\mathrm{kN}) \\
\text { sem considerar DPC }\end{array}$ & $\begin{array}{c}\text { Cortante }(\mathrm{kN}) \\
\text { considerando DPC }\end{array}$ & $\begin{array}{c}\text { Variação } \\
(\%)\end{array}$ \\
\hline 1 & $-5,092$ & $+0,029$ & - \\
\hline 2 & $+6,14$ & $+7,48$ & $+21,74$ \\
\hline 3 & $+14,74$ & $+15,98$ & $+8,39$ \\
\hline 4 & $+20,69$ & $+21,45$ & $+3,68$ \\
\hline 5 & $+24,70$ & $+25,19$ & $+1,97$ \\
\hline 6 & $+27,13$ & $+27,41$ & $+1,05$ \\
\hline 7 & $+28,32$ & $+28,47$ & $+0,52$ \\
\hline 8 & $+28,58$ & $+28,63$ & $+0,19$ \\
\hline 9 & $+28,15$ & $+28,14$ & $-0,05$ \\
\hline 10 & $+27,27$ & $+27,21$ & $-0,22$ \\
\hline 11 & $+26,15$ & $+26,04$ & $-0,40$ \\
\hline 12 & $+24,94$ & $+24,80$ & $-0,57$ \\
\hline 13 & $+24,07$ & $+23,82$ & $-1,02$ \\
\hline 14 & $+22,41$ & $+22,27$ & $-0,62$ \\
\hline 15 & $+28,87$ & $+27,94$ & $-3,21$ \\
\hline
\end{tabular}




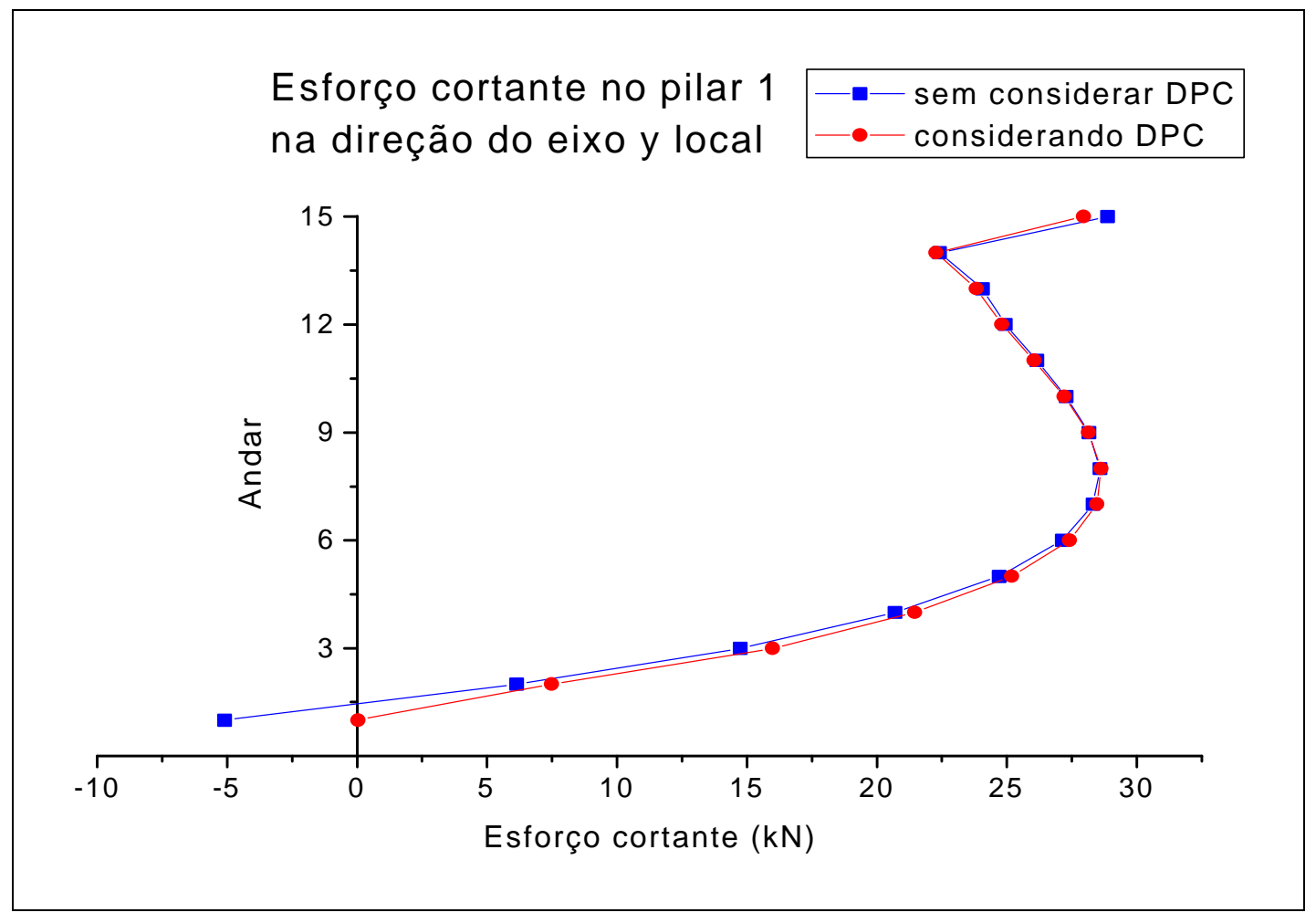

Figura 40 - Esforço cortante no pilar 1 na direção do eixo y local

Tabela 15 - Bimomento na extremidade inferior do núcleo

\begin{tabular}{|c|c|c|c|}
\hline Andar & $\begin{array}{c}\text { Bimomento }\left(\mathrm{kN} . \mathrm{m}^{2}\right) \\
\text { sem considerar DPC }\end{array}$ & $\begin{array}{c}\text { Bimomento }\left(\mathrm{kN} . \mathrm{m}^{2}\right) \\
\text { considerando DPC }\end{array}$ & $\begin{array}{c}\text { Variação } \\
(\%)\end{array}$ \\
\hline 1 & $-1.933,98$ & $-1.898,59$ & $-1,83$ \\
\hline 2 & $+118,56$ & $+99,47$ & $-16,11$ \\
\hline 3 & $+102,67$ & $+102,68$ & $-0,01$ \\
\hline 4 & $+133,63$ & $+130,96$ & $-2,00$ \\
\hline 5 & $+109,58$ & $+108,81$ & $-0,70$ \\
\hline 6 & $+95,90$ & $+95,50$ & $-0,41$ \\
\hline 7 & $+83,39$ & $+74,49$ & $+0,12$ \\
\hline 8 & $+74,16$ & $+67,93$ & $+0,51$ \\
\hline 9 & $+67,36$ & $+63,48$ & $+0,85$ \\
\hline 10 & $+62,81$ & $+61,04$ & $+1,07$ \\
\hline 11 & $+60,36$ & $+60,36$ & $+1,12$ \\
\hline 12 & $+59,67$ & $+62,03$ & $+1,15$ \\
\hline 13 & $+61,72$ & $+62,63$ & $+1,67$ \\
\hline 14 & $+61,59$ & $+74,32$ & $-3,17$ \\
\hline 15 & $+76,76$ & & \\
\hline
\end{tabular}




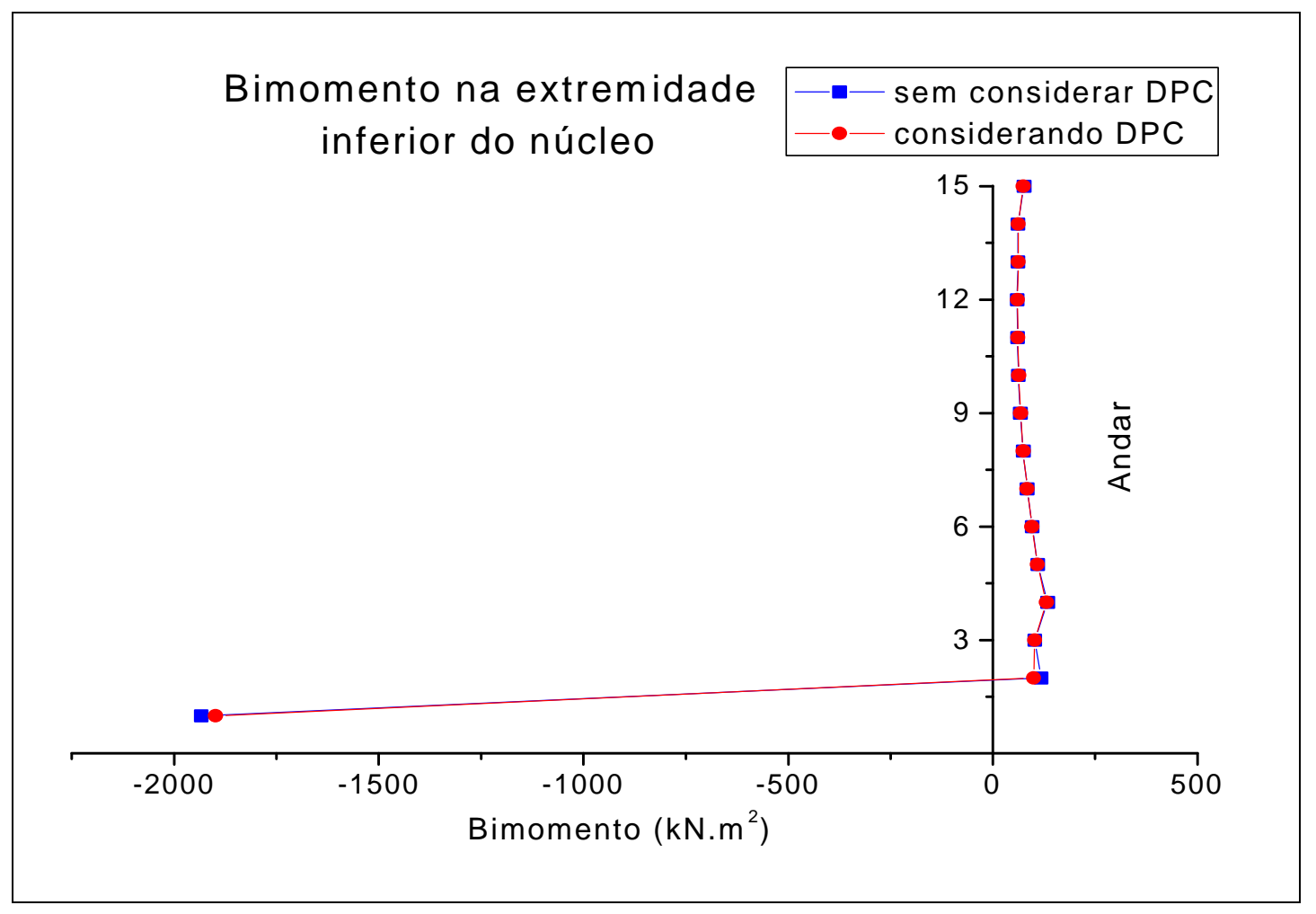

Figura 41 - Bimomento na extremidade inferior do núcleo

A análise dos resultados obtidos neste exemplo permite fazer as seguintes considerações:

- Ao considerar o efeito da deformação pelo cortante, nota-se um aumento da translação do nó mestre da laje na direção $Y_{g}$, que é mais pronunciado nos pavimentos inferiores. Nota-se, também, que os resultados aproximam-se mais dos obtidos por Silva.

- Nota-se uma diferença muito pequena nos momentos fletores obtidos no núcleo com e sem deformação por esforço cortante. Já nos pilares, a variação dos momentos fletores pode ser pequena, como no caso do pilar 3 (tabela 9) ou significativa, como no pilar 2 no primeiro pavimento (tabela 10 ).

- Nota-se que a consideração da deformação por cortante provoca uma redução do esforço cortante no núcleo estrutural e, naturalmente, um aumento do esforço médio nos pilares. Dependendo da disposição dos elementos de contraventamento, a variação do cortante em determinados pilares pode ser bem grande, como ocorreu com o pilar 1 no primeiro andar. Neste pilar o esforço cortante passou de 5,092 $\mathrm{kN}$ no sentido contrário ao carregamento para $0,029 \mathrm{kN}$ no 
sentido do carregamento. Essa redistribuição de esforço cortante entre o núcleo e os pilares é muito mais pronunciada nos pavimentos mais baixos.

- Verifica-se também que os esforços cortantes obtidos no núcleo com consideração da deformação por cortante estão mais próximos dos obtidos por Silva. 


\section{4 - Exemplo 4}

Este exemplo foi extraído de BECKER (1989), consistindo de um edifício de 9 andares e pé-direito $3,0 \mathrm{~m}$, cuja planta dos pavimentos-tipo é apresentada na figura 42 .

Adotaram-se para os módulos de elasticidade longitudinal e transversal do material que compõe a estrutura os valores $200 \mathrm{tf} / \mathrm{cm}^{2}$ e $80 \mathrm{tf} / \mathrm{cm}^{2}$, respectivamente.

As características geométricas dos elementos que compõem a estrutura são:

- Os pilares 1, 2, 3, 7, 8, 9, 10, 11 e 12 possuem seção transversal de dimensão $30 \mathrm{~cm}$ x $60 \mathrm{~cm}$ e o pilar 5 possui seção transversal $30 \mathrm{~cm}$ x $90 \mathrm{~cm}$.

- As paredes do núcleo têm $20 \mathrm{~cm}$ de espessura

- A dimensão da seção transversal das vigas pilar-pilar, das vigas pilar-núcleo e dos lintéis é $20 \mathrm{~cm}$ x $40 \mathrm{~cm}$.

O carregamento aplicado é uma força horizontal, uniformemente distribuída ao longo da altura, na direção e sentido do eixo $Z_{\mathrm{g}}$ e de valor 866,67 kgf $/ \mathrm{m}$.

Os fatores de forma da seção do núcleo são: $\alpha_{\mathrm{yy}}=2,78328$ e $\alpha_{\mathrm{zz}}=4,46600$ quando calculados pelo processo geométrico e $\alpha_{\mathrm{yy}}=2,95768$ e $\alpha_{\mathrm{zz}}=4,49847$, quando calculados pelo processo de energia. Utilizaram-se neste exemplo os fatores de forma obtidos pelo processo de igualdade de energia.

Após a figura 42, apresentam-se tabelas e gráficos que apresentam os deslocamentos e esforços para o carregamento aplicado, nas hipóteses de admitir ou desprezar a deformação devida ao cisalhamento pelo cortante.

Os resultados de deslocamentos dos nós mestres (tabela 16), esforços cortantes em núcleos e pilares (tabela 17) e momentos fletores no núcleo (tabela 19) foram apresentados por Becker em sua dissertação e coincidem exatamente com os obtidos pelo CEASO 01 inalterado, já que ambos não levam em conta a distorção tanto no núcleo quanto nos pilares. 


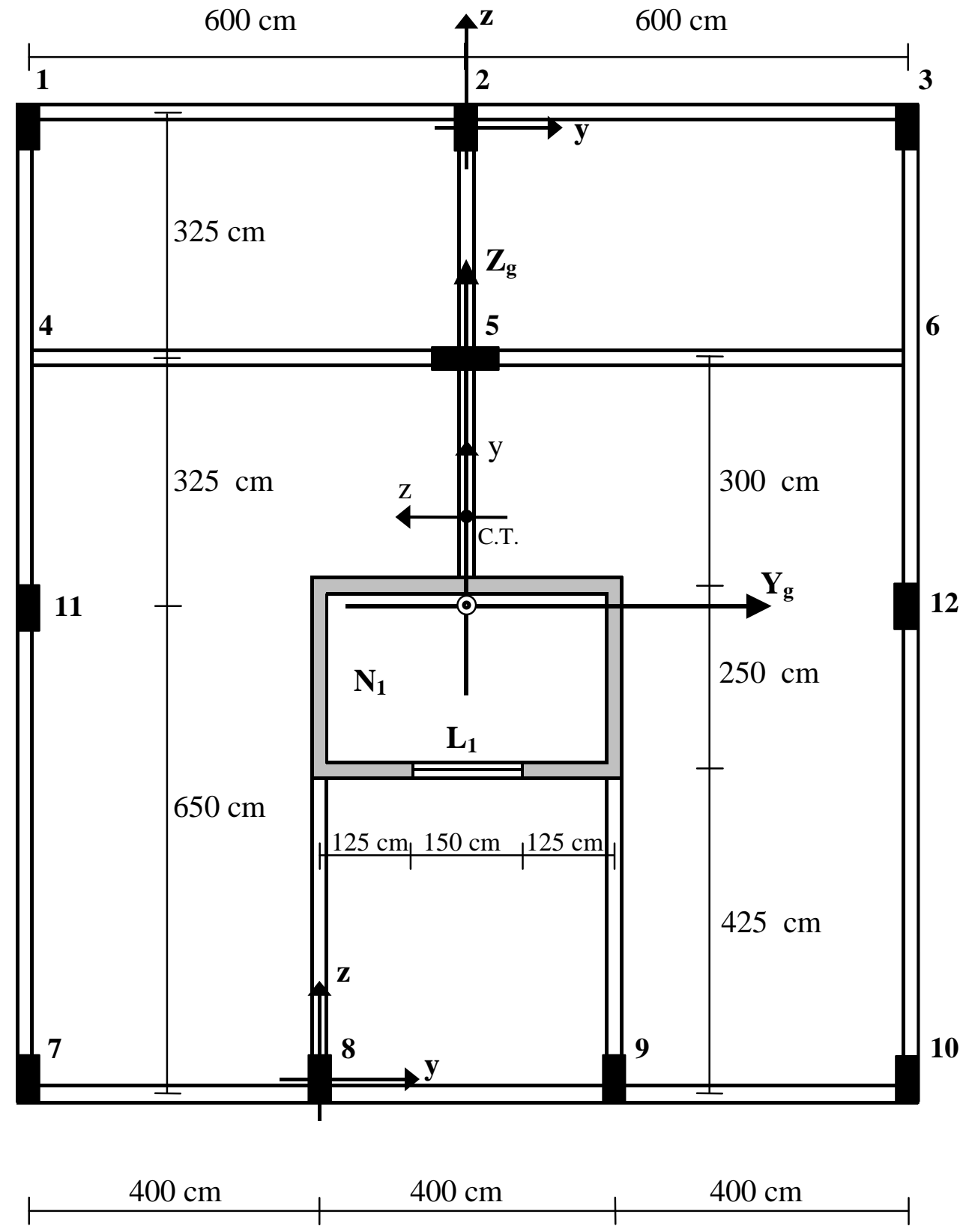

Figura 42 - Planta do pavimento-tipo do exemplo 4 
Tabela 16 - Translação do nó mestre da laje na direção de $\mathrm{Z}_{\mathrm{g}}$

\begin{tabular}{|c|c|c|c|c|}
\hline Andar & $\begin{array}{c}\text { Desloc. }(\mathrm{cm}) \\
\text { sem DPC }\end{array}$ & $\begin{array}{c}\text { Desloc. }(\mathrm{cm}) \\
\text { DPC no núcleo }\end{array}$ & $\begin{array}{c}\text { Desloc. }(\mathrm{cm}) \\
\text { DPC nos pilares }\end{array}$ & $\begin{array}{c}\text { Desloc. }(\mathrm{cm}) \\
\text { DPC núcleo/pilares }\end{array}$ \\
\hline 1 & 0,017 & 0,026 & 0,017 & 0,026 \\
\hline 2 & 0,060 & 0,077 & 0,061 & 0,077 \\
\hline 3 & 0,122 & 0,144 & 0,122 & 0,145 \\
\hline 4 & 0,193 & 0,221 & 0,194 & 0,221 \\
\hline 5 & 0,270 & 0,301 & 0,271 & 0,302 \\
\hline 6 & 0,349 & 0,381 & 0,349 & 0,382 \\
\hline 7 & 0,426 & 0,459 & 0,427 & 0,460 \\
\hline 8 & 0,500 & 0,534 & 0,502 & 0,535 \\
\hline 9 & 0,573 & 0,605 & 0,575 & 0,607 \\
\hline
\end{tabular}

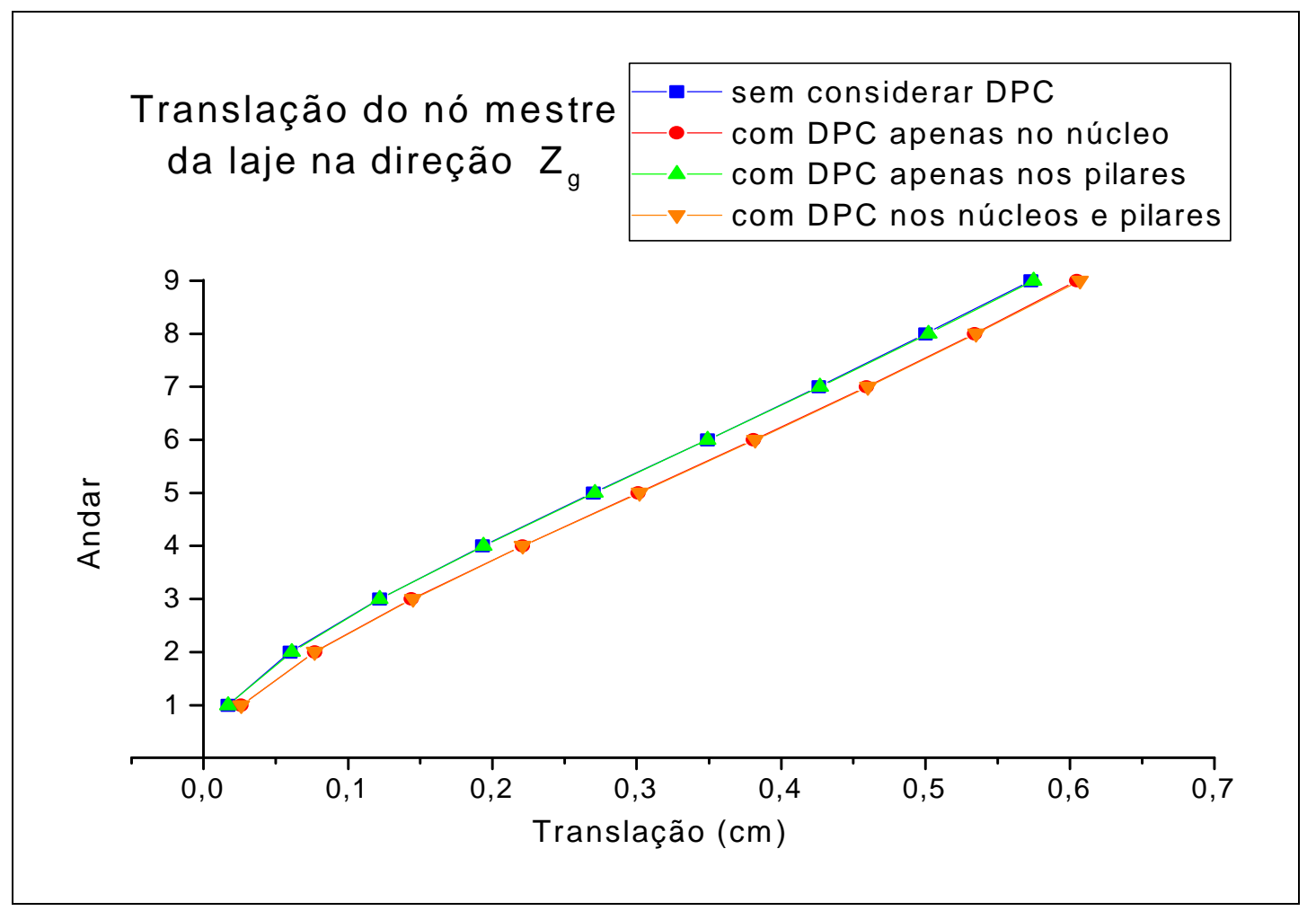

Figura 43 - Translação do nó mestre da laje na direção $Z_{g}$ 
Tabela 17 - Distribuição do esforço cortante na direção do eixo $\mathrm{Z}_{\mathrm{g}}$ entre o núcleo e os pilares sem considerar deformação por cortante

\begin{tabular}{|c|c|c|c|}
\hline Andar & $\begin{array}{c}\text { Esf. Cortante (tf) } \\
\text { Núcleo }\end{array}$ & $\begin{array}{c}\text { Esf. Cortante (tf) } \\
\text { Pilares }\end{array}$ & $\begin{array}{c}\text { Esf. Núcleo / Esf. Pav. } \\
(\%)\end{array}$ \\
\hline 1 & 21,077 & 1,023 & 95,37 \\
\hline 2 & 17,590 & 1,910 & 90,21 \\
\hline 3 & 14,458 & 2,442 & 85,55 \\
\hline 4 & 11,567 & 2,733 & 80,89 \\
\hline 5 & 8,859 & 2,841 & 75,71 \\
\hline 6 & 6,305 & 2,795 & 69,28 \\
\hline 7 & 3,740 & 2,760 & 57,54 \\
\hline 8 & 1,608 & 2,292 & 41,24 \\
\hline 9 & $-2,249$ & 3,549 & - \\
\hline
\end{tabular}

Tabela 18 - Distribuição do esforço cortante na direção do eixo $\mathrm{Z}_{\mathrm{g}}$ entre o núcleo e os pilares considerando deformação por cortante

\begin{tabular}{|c|c|c|c|}
\hline Andar & $\begin{array}{c}\text { Esf. Cortante (tf) } \\
\text { Núcleo }\end{array}$ & $\begin{array}{c}\text { Esf. Cortante (tf) } \\
\text { Pilares }\end{array}$ & $\begin{array}{c}\text { Esf. Núcleo / Esf. Pav. } \\
(\%)\end{array}$ \\
\hline 1 & 19,444 & 2,656 & 87,98 \\
\hline 2 & 17,639 & 1,861 & 90,46 \\
\hline 3 & 14,267 & 2,633 & 84,42 \\
\hline 4 & 11,468 & 2,832 & 80,20 \\
\hline 5 & 8,789 & 2,911 & 75,12 \\
\hline 6 & 6,256 & 2,844 & 68,75 \\
\hline 7 & 3,762 & 2,738 & 57,88 \\
\hline 8 & 1,529 & 2,371 & 39,21 \\
\hline 9 & $-2,039$ & 3,339 & - \\
\hline
\end{tabular}




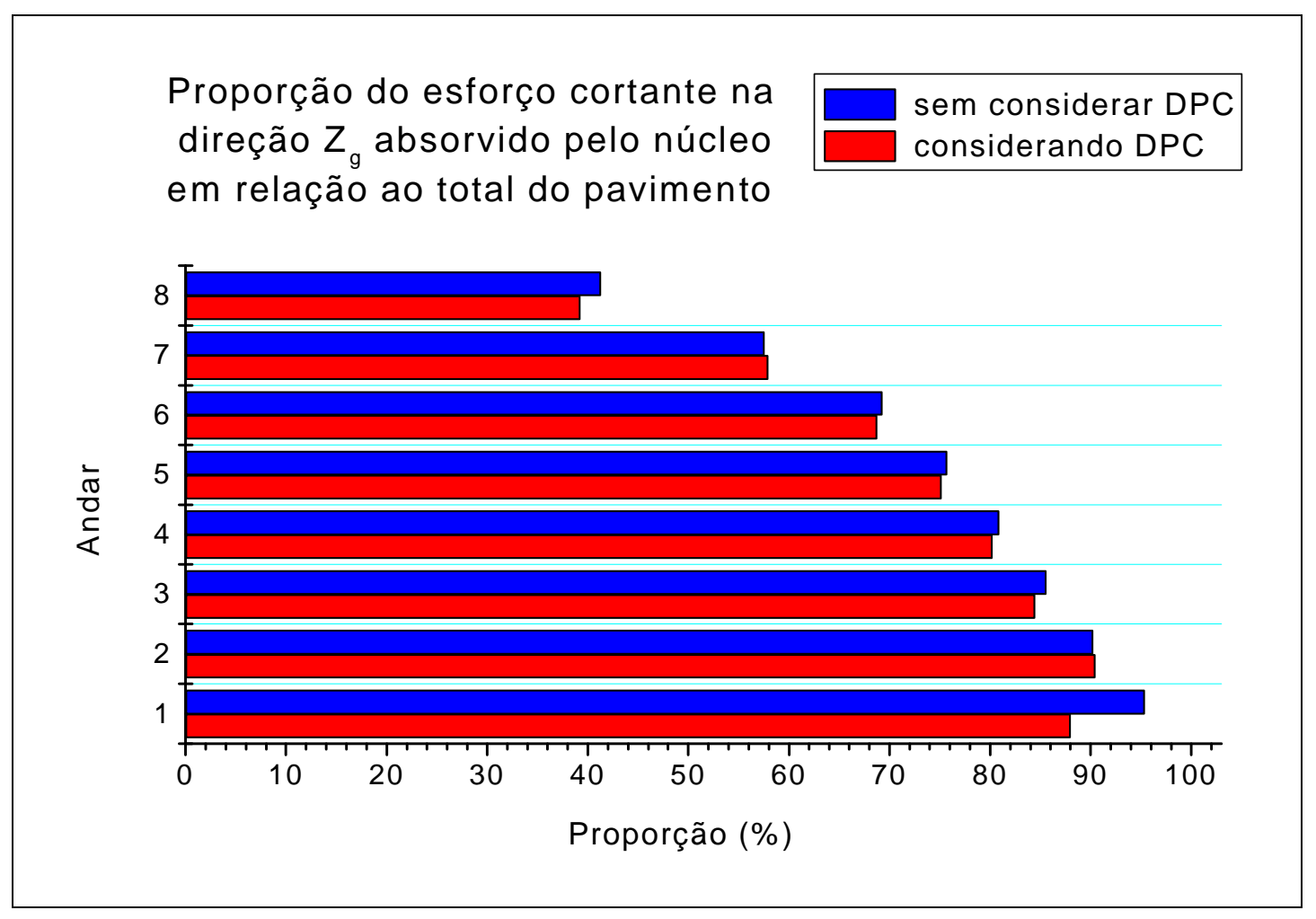

Figura 44 - Proporção do esforço cortante na direção $Z_{g}$ absorvido pelo núcleo em relação ao total do pavimento

Tabela 19 - Momento fletor em torno do eixo z local do núcleo

\begin{tabular}{|c|c|c|c|c|}
\hline \multirow{2}{*}{ Andar } & \multicolumn{2}{|c|}{$\mathrm{M}_{\mathrm{z}}(\mathrm{tf} . \mathrm{cm})-$ sem DPC } & \multicolumn{2}{c|}{$\mathrm{M}_{\mathrm{z}}(\mathrm{tf} . \mathrm{cm})-$ com DPC } \\
\cline { 2 - 5 } & Topo & Base & Topo & Base \\
\hline 1 & $+14.480,74$ & $-20.803,91$ & $+14.400,39$ & $-20.233,60$ \\
\hline 2 & $+9.426,87$ & $-14.703,99$ & $+9.348,64$ & $-14.640,40$ \\
\hline 3 & $+5.463,64$ & $-9.801,14$ & $+5.451,10$ & $-9.731,11$ \\
\hline 4 & $+2.460,80$ & $-5.930,80$ & $+2.483,02$ & $-5.923,52$ \\
\hline 5 & $+318,89$ & $-2.976,46$ & $+364,38$ & $-3.001,18$ \\
\hline 6 & $-1.040,99$ & $-850,39$ & $-980,40$ & $-896,40$ \\
\hline 7 & $-1.637,14$ & $+515,07$ & $-1.584,47$ & $+455,74$ \\
\hline 8 & $-1.612,99$ & $+1.130,51$ & $-1.538,90$ & $+1.080,13$ \\
\hline 9 & $-444,28$ & $+1.119,01$ & $-438,05$ & $+1.049,88$ \\
\hline
\end{tabular}


Tabela 20 - Esforço cortante na direção do eixo z local do pilar 2

\begin{tabular}{|c|c|c|c|}
\hline Andar & $\begin{array}{c}\text { Esf. Cortante (tf) } \\
\text { sem DPC }\end{array}$ & $\begin{array}{c}\text { Esf. Cortante (tf) } \\
\text { com DPC }\end{array}$ & $\begin{array}{c}\text { Variação } \\
(\%)\end{array}$ \\
\hline 1 & 0,102 & 0,279 & $+173,90$ \\
\hline 2 & 0,185 & 0,184 & $-0,39$ \\
\hline 3 & 0,234 & 0,256 & $+9,70$ \\
\hline 4 & 0,260 & 0,272 & $+4,69$ \\
\hline 5 & 0,269 & 0,277 & $+3,13$ \\
\hline 6 & 0,264 & 0,269 & $+2,04$ \\
\hline 7 & 0,256 & 0,254 & $-0,82$ \\
\hline 8 & 0,220 & 0,227 & $+3,14$ \\
\hline 9 & 0,311 & 0,287 & $+7,59$ \\
\hline
\end{tabular}

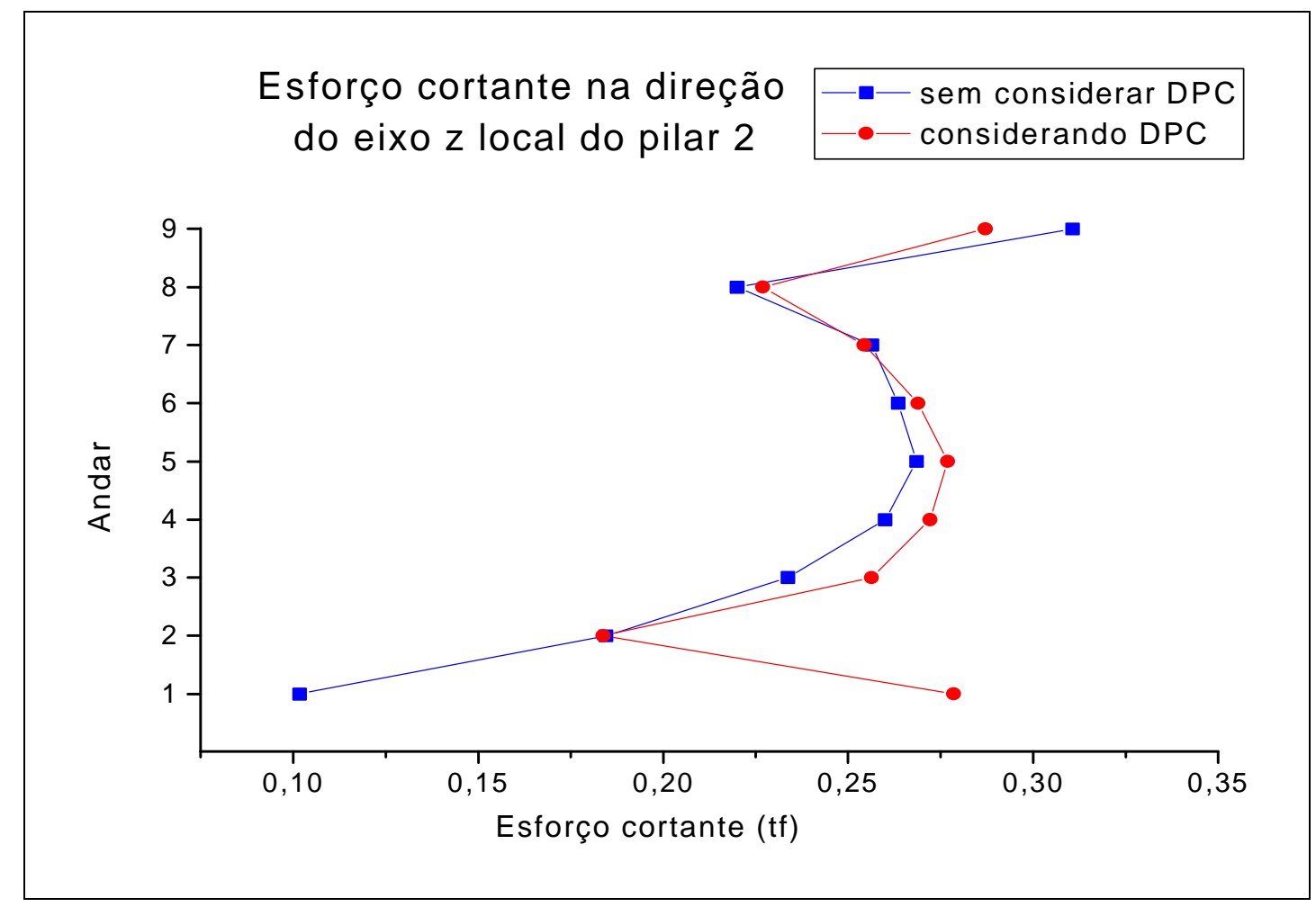

Figura 45 - Esforço cortante na direção do eixo z local do pilar 2 
Tabela 21 - Momento fletor em torno do eixo y local do pilar 2

\begin{tabular}{|c|c|c|c|c|}
\hline \multirow{2}{*}{ Andar } & \multicolumn{2}{|c|}{$\mathrm{M}_{\mathrm{y}}(\mathrm{tf} . \mathrm{cm})-\mathrm{sem}$ DPC } & \multicolumn{2}{c|}{$\mathrm{M}_{\mathrm{y}}(\mathrm{tf} . \mathrm{cm})-$ com DPC } \\
\cline { 2 - 5 } & Topo & Base & Topo & Base \\
\hline 1 & $-20,19$ & $+50,70$ & $-4,80$ & $+88,36$ \\
\hline 2 & $+3,24$ & $+52,11$ & $+7,59$ & $+47,54$ \\
\hline 3 & $+19,59$ & $+50,52$ & $+24,29$ & $+52,61$ \\
\hline 4 & $+30,43$ & $+47,54$ & $+33,83$ & $+47,80$ \\
\hline 5 & $+36,89$ & $+43,67$ & $+39,43$ & $+43,65$ \\
\hline 6 & $+39,58$ & $+39,48$ & $+41,65$ & $+39,02$ \\
\hline 7 & $+40,87$ & $+36,05$ & $+41,47$ & $+34,81$ \\
\hline 8 & $+34,31$ & $+31,69$ & $+37,06$ & $+31,02$ \\
\hline 9 & $+54,92$ & $+38,30$ & $+52,39$ & $+33,76$ \\
\hline
\end{tabular}

Momento fletor em torno do - sem considerar DPC
eixo y local no topo do pilar $2 \longrightarrow$ considerando DPC

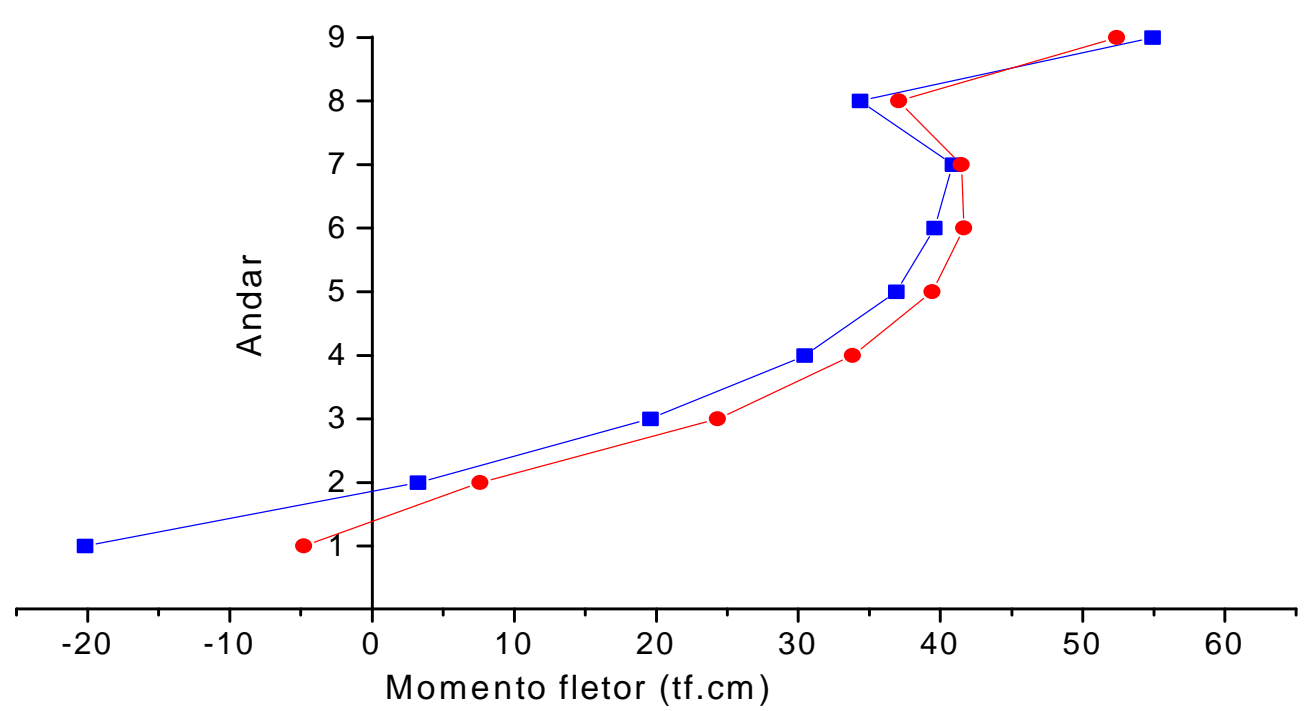

Figura 46 - Momento fletor em torno do eixo y local no topo do pilar 2 
Tabela 22 - Esforço cortante na direção do eixo z local do pilar 8

\begin{tabular}{|c|c|c|c|}
\hline Andar & $\begin{array}{c}\text { Esf. Cortante (tf) } \\
\text { sem DPC }\end{array}$ & $\begin{array}{c}\text { Esf. Cortante (tf) } \\
\text { com DPC }\end{array}$ & $\begin{array}{c}\text { Variação } \\
(\%)\end{array}$ \\
\hline 1 & 0,119 & 0,286 & $+140,70$ \\
\hline 2 & 0,228 & 0,213 & $-6,47$ \\
\hline 3 & 0,292 & 0,306 & $+4,90$ \\
\hline 4 & 0,327 & 0,332 & $+1,58$ \\
\hline 5 & 0,339 & 0,342 & $+1,00$ \\
\hline 6 & 0,332 & 0,334 & $+0,83$ \\
\hline 7 & 0,329 & 0,324 & $-1,40$ \\
\hline 8 & 0,263 & 0,272 & $+3,65$ \\
\hline 9 & 0,439 & 0,417 & $-4,98$ \\
\hline
\end{tabular}

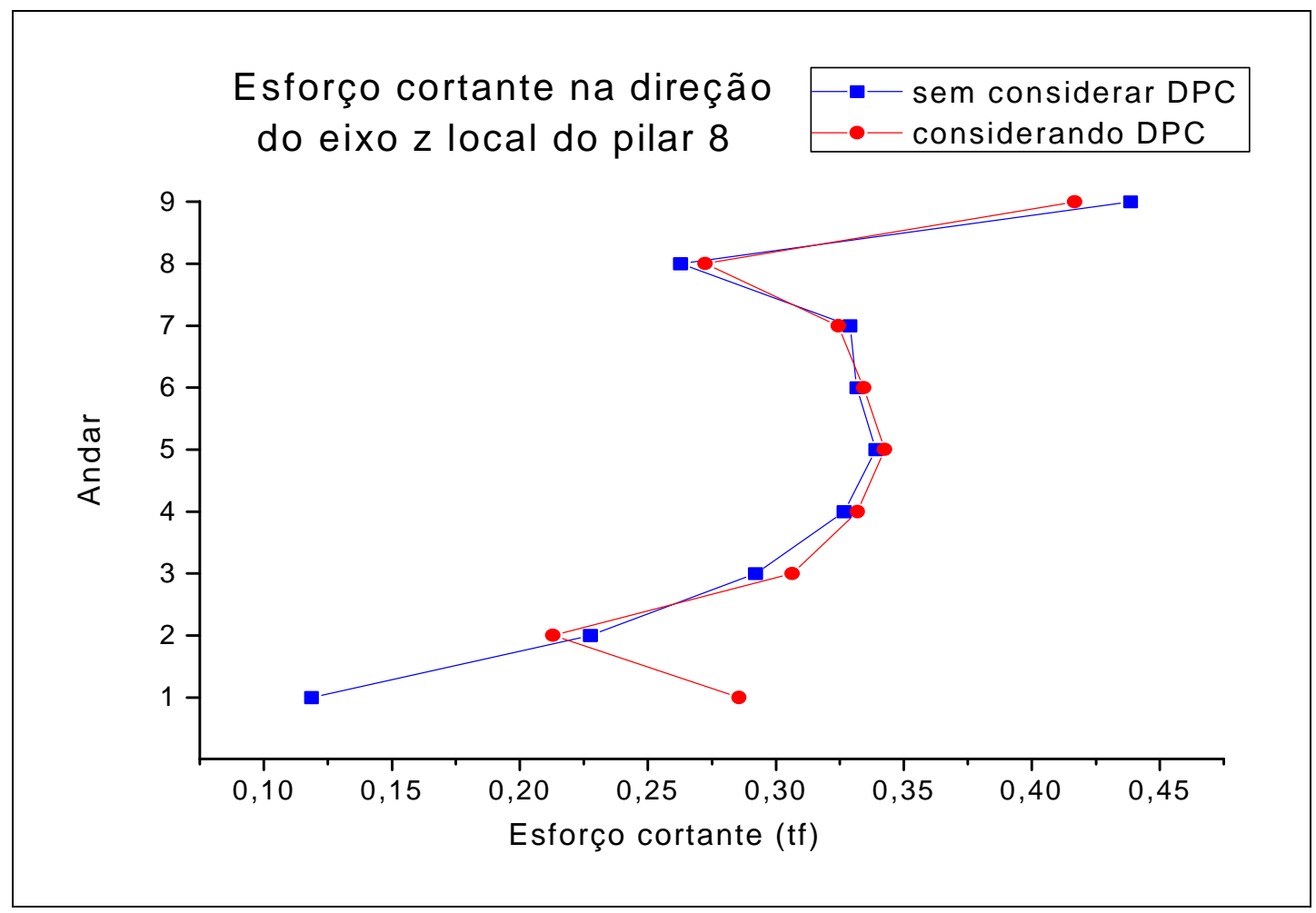

Figura 47 - Esforço cortante na direção do eixo z local do pilar 8. 
Tabela 23 - Momento fletor em torno do eixo y local do pilar 8

\begin{tabular}{|c|c|c|c|c|}
\hline \multirow{2}{*}{ Andar } & \multicolumn{2}{|c|}{$\mathrm{M}_{\mathrm{y}}(\mathrm{tf} . \mathrm{cm})-\mathrm{sem}$ DPC } & \multicolumn{2}{c|}{$\mathrm{M}_{\mathrm{y}}(\mathrm{tf} . \mathrm{cm})-$ com DPC } \\
\cline { 2 - 5 } & Topo & Base & Topo & Base \\
\hline 1 & $-16,80$ & $+52,40$ & $-3,339$ & $+89,03$ \\
\hline 2 & $+10,16$ & $+58,12$ & $+12,79$ & $+51,07$ \\
\hline 3 & $+28,63$ & $+58,96$ & $+32,11$ & $+59,77$ \\
\hline 4 & $+40,58$ & $+57,43$ & $+43,01$ & $+56,55$ \\
\hline 5 & $+47,52$ & $+54,18$ & $+49,35$ & $+53,37$ \\
\hline 6 & $+49,61$ & $+49,87$ & $+51,36$ & $+48,94$ \\
\hline 7 & $+52,17$ & $+46,53$ & $+52,35$ & $+44,97$ \\
\hline 8 & $+38,82$ & $+40,01$ & $+42,13$ & $+39,57$ \\
\hline 9 & $+80,25$ & $+51,32$ & $+78,28$ & $+46,75$ \\
\hline
\end{tabular}

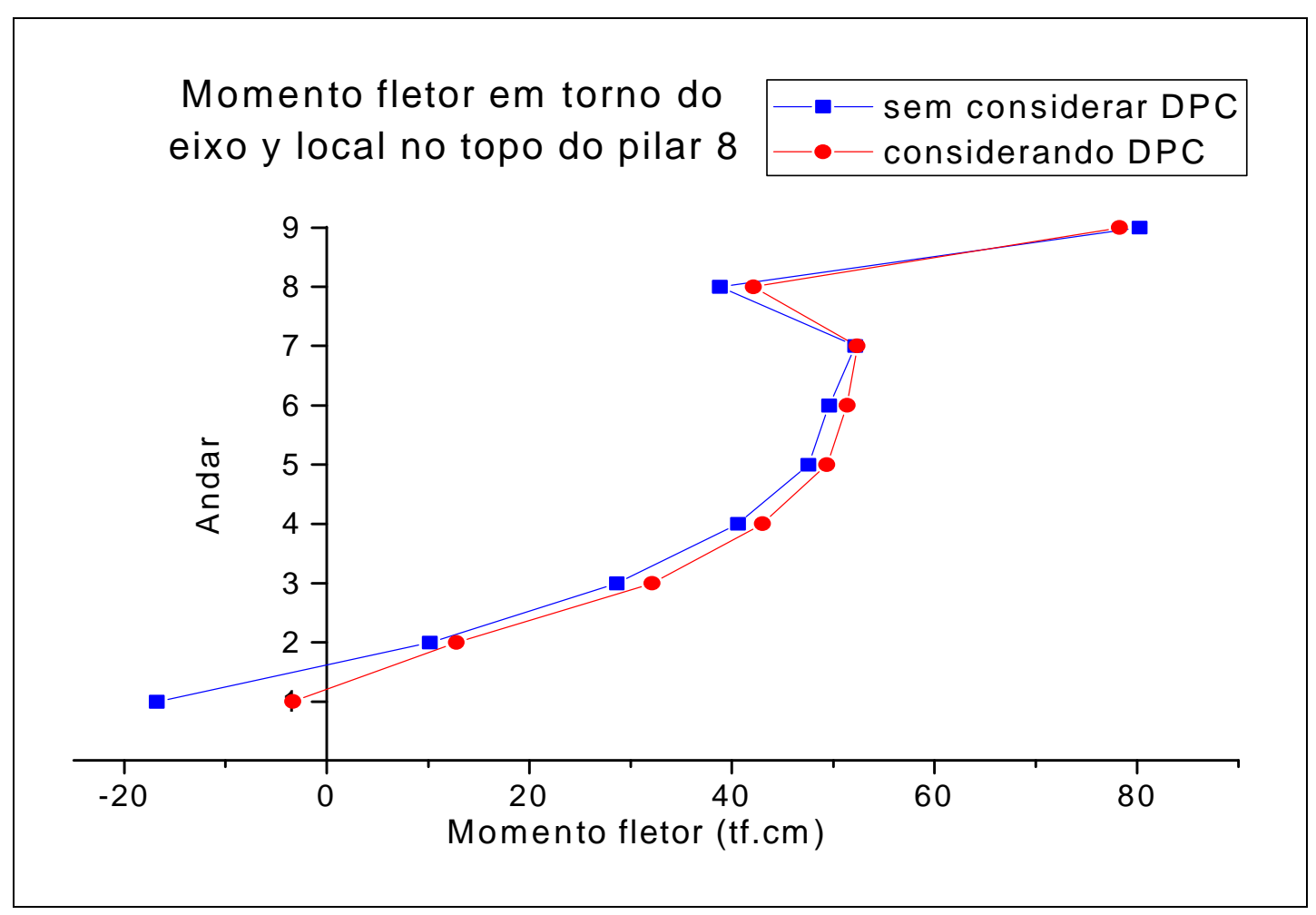

Figura 48 - Momento fletor em torno do eixo y local no topo do pilar 8 
A análise dos resultados apresentados permite que se façam as seguintes considerações:

- A consideração da deformação pelo cortante provoca um aumento da translação do nó mestre das lajes na direção de $\mathrm{Z}_{\mathrm{g}}$. Como pode ser visto na tabela 16, este aumento dos deslocamentos é devido quase totalmente à consideração da distorção no núcleo resistente, sendo mínima a contribuição dos pilares.

- Nota-se que o esforço cortante no núcleo se reduz ao considerar a deformação pelo cortante, principalmente no $1^{\circ}$ pavimento. O núcleo que absorvia $95,37 \%$ do esforço cortante total no $1^{\circ}$ pavimento passou a absorver $87,98 \%$, como se pode ver nas tabelas 17 e 18 .

- Também nas tabelas 17 e 18, pode-se ver que a soma de esforços cortantes absorvidos por todos os pilares do $1^{\circ}$ pavimento tem um aumento de $159,63 \%$, passando de 1,023 tf para 2,656 tf . Em alguns pilares, o aumento é ainda maior, como é o caso do pilar 2 ( tabela 20 ), que teve esse esforço aumentado em 173,90\%. Nos demais pavimentos, constata-se que a variação é bem mais discreta.

- A consideração da deformação por cortante altera muito pouco os momentos fletores do núcleo resistente, como se pode ver na tabela 19.

- Com relação aos momentos fletores nos pilares, verifica-se que, ao menos no $1^{\circ}$ andar, ocorreram variações significativas ao se considerar a deformação por cortante, como pode ser visto nas tabelas 21 (pilar 2) e 23 (pilar 8). 


\section{5 - Exemplo 5}

Este exemplo consiste num edifício de 25 andares e pé-direito $2,80 \mathrm{~m}$, cuja planta dos pavimentos-tipo é apresentada na figura 49. Ele foi desenvolvido por este autor, baseado na observação de características estruturais de edifícios construídos.

Adotaram-se para os módulos de elasticidade longitudinal e transversal do material que compõe a estrutura os valores $2.800 \mathrm{kN} / \mathrm{cm}^{2}$ e $1.120 \mathrm{kN} / \mathrm{cm}^{2}$, respectivamente. As características geométricas dos elementos que compõem a estrutura são as seguintes:

Tabela 24 - Dimensões dos pilares

\begin{tabular}{|c|c|}
\hline Pilares & Dimensões \\
\hline $1,4,17,20$ & $50 \mathrm{~cm} \times 60 \mathrm{~cm}$ \\
\hline $2,3,18,19$ & $20 \mathrm{~cm} \times 60 \mathrm{~cm}$ \\
\hline $6,8,13,15$ & $25 \mathrm{~cm} \times 70 \mathrm{~cm}$ \\
\hline $5,9,12,16$ & $30 \mathrm{~cm} \times 90 \mathrm{~cm}$ \\
\hline 10,11 & $20 \mathrm{~cm} \times 80 \mathrm{~cm}$ \\
\hline 7,14 & $25 \mathrm{~cm} \times 80 \mathrm{~cm}$ \\
\hline
\end{tabular}

Tabela 25 - Dimensões das vigas

\begin{tabular}{|c|c|}
\hline Vigas & Base $\times$ altura \\
\hline $1,2,11,12$ & $20 \mathrm{~cm} \times 65 \mathrm{~cm}$ \\
\hline $3,4,5,6,7,8,9,10,17,18$, & $15 \mathrm{~cm} \times 30 \mathrm{~cm}$ \\
\hline $13,14,15,16,22,23,24,25$ & $20 \mathrm{~cm} \times 40 \mathrm{~cm}$ \\
\hline 19 & $20 \mathrm{~cm} \times 50 \mathrm{~cm}$ \\
\hline
\end{tabular}

As paredes dos núcleos têm $25 \mathrm{~cm}$ de espessura e os fatores de forma dos núcleos são: $\alpha_{\mathrm{yy}}=2,49583$ e $\alpha_{\mathrm{zz}}=2,82757$ quando calculados pelo processo geométrico e $\alpha_{\mathrm{yy}}=2,66345$ e $\alpha_{\mathrm{zz}}=2,84699$, quando calculados pelo processo energético. Estes dois últimos foram os valores adotados na análise deste exemplo.

As forças derivadas da ação do vento foram calculadas conforme a NBR 6123, da ASSOCIAÇÃO BRASILEIRA DE NORMAS TÉCNICAS - ABNT (1987), sendo admitidos: 
- Ocorrência de ventos de baixa turbulência.

- Velocidade básica do vento de $45 \mathrm{~m} / \mathrm{s}$.

- Edificação situada no nível superior de um talude de 40 m, a uma distância de $120 \mathrm{~m}$ do início da declividade.

- Terreno classificado quanto à rugosidade como de categoria IV.

- Edificação é de classe $\mathrm{C}$, devido às suas dimensões e classificada como do Grupo 2, quanto à sua utilização.

- Considera-se excentricidade da carga de vento de 7,5\% da dimensão perpendicular à incidência do mesmo.

Aplicam-se em cada laje forças horizontais na direção do eixo $Z_{g}$ e momentos torçores em torno do eixo $\mathrm{X}_{\mathrm{g}}$, conforme mostrado na tabela 26 .

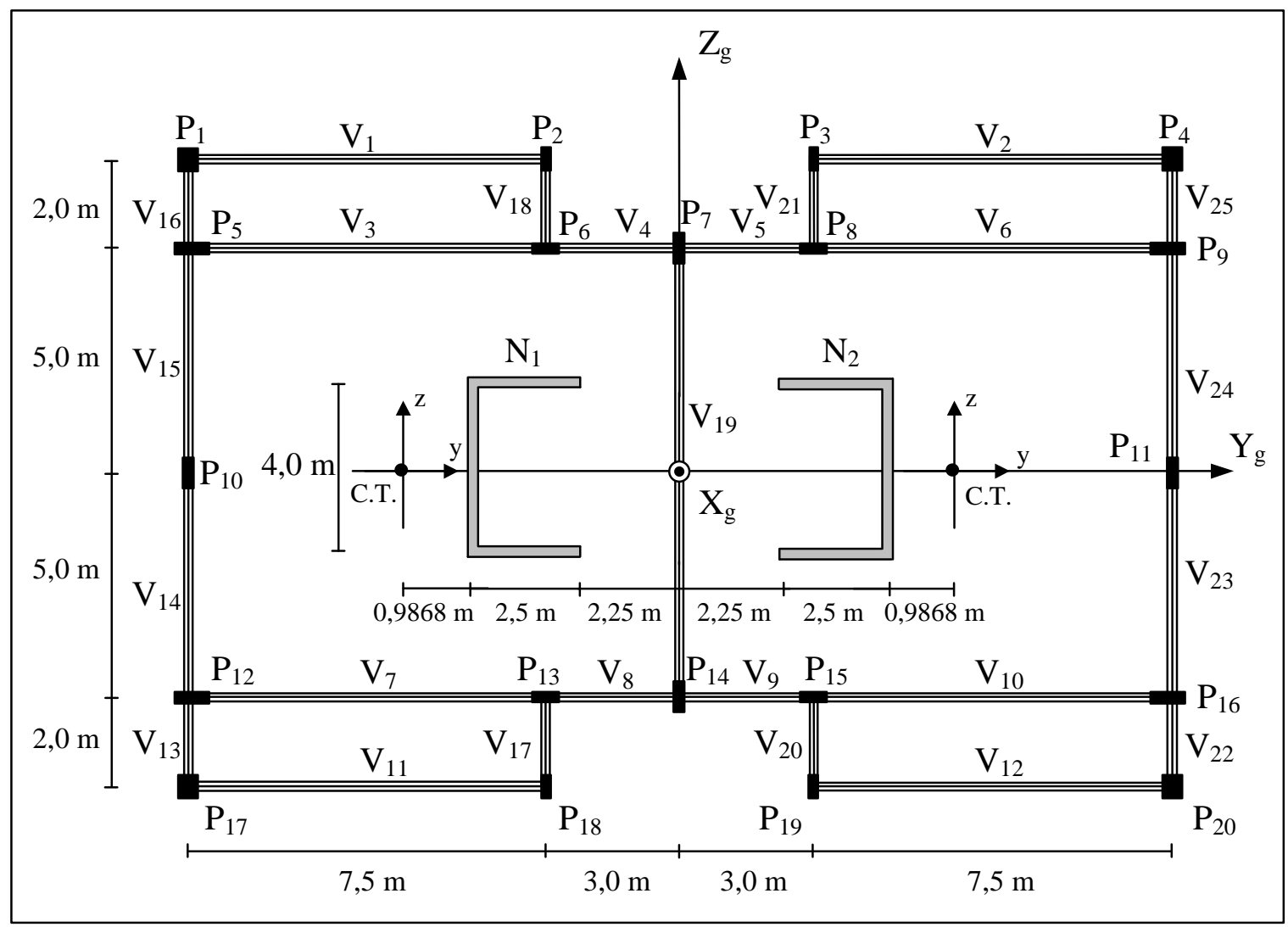

Figura 49 - Planta do pavimento-tipo do exemplo 5 
Tabela 26 - Carregamento aplicado à estrutura

\begin{tabular}{|c|c|c|}
\hline Laje & $\mathrm{F}_{\mathrm{z}}(\mathrm{kN})$ & $\mathrm{M}_{\mathrm{x}}(\mathrm{kN} \cdot \mathrm{cm})$ \\
\hline 1 & 80,70 & $13.315,49$ \\
\hline 2 & 88,95 & $14.677,28$ \\
\hline 3 & 93,91 & $15.494,71$ \\
\hline 4 & 97,40 & $16.070,86$ \\
\hline 5 & 100,05 & $16.507,48$ \\
\hline 6 & 102,13 & $16.852,08$ \\
\hline 7 & 103,82 & $17.130,94$ \\
\hline 8 & 105,21 & $17.360,25$ \\
\hline 9 & 106,37 & $17.550,71$ \\
\hline 10 & 107,33 & $17.709,83$ \\
\hline 11 & 108,14 & $17.843,06$ \\
\hline 12 & 108,82 & $17.954,52$ \\
\hline 13 & 109,38 & $18.047,37$ \\
\hline 14 & 109,84 & $18.124,13$ \\
\hline 15 & 110,22 & $18.186,83$ \\
\hline 16 & 110,53 & $18.237,12$ \\
\hline 17 & 110,77 & $18.276,38$ \\
\hline 18 & 110,94 & $18.305,76$ \\
\hline 19 & 111,07 & $18.326,24$ \\
\hline 20 & 111,14 & $18.338,66$ \\
\hline 21 & 111,17 & $18.343,75$ \\
\hline 22 & 111,16 & $18.342,13$ \\
\hline 23 & 111,12 & $18.334,37$ \\
\hline 24 & 111,04 & $18.320,95$ \\
\hline 25 & 55,46 & $9.151,15$ \\
\hline & & \\
\hline
\end{tabular}

Apresentam-se a seguir tabelas e gráficos com os resultados obtidos neste exemplo.

Verifica-se que o valor do deslocamento do nó mestre da última laje $(17,11 \mathrm{~cm})$ é cerca de $22 \%$ superior ao valor limite previsto pela NB-1 da ABNT ${ }^{* *}$ apud CARMO (1995), que é de H/500, sendo H a altura do edifício. Apesar disso, acredita-se que este exemplo seja representativo do comportamento dos edifícios altos usualmente projetados.

** ASSOCIAÇÃO BRASILEIRA DE NORMAS TÉCNICAS (1994). NB-1: Texto base para revisão 
Tabela 27 - Translação $(\mathrm{cm})$ do nó mestre da laje na direção de $Z_{\mathrm{g}}$

\begin{tabular}{|c|c|c|}
\hline Laje & sem considerar DPC & considerando DPC \\
\hline 1 & 0,06714 & 0,1026 \\
\hline 2 & 0,2584 & 0,3286 \\
\hline 3 & 0,5594 & 0,6606 \\
\hline 4 & 0,9569 & 1,086 \\
\hline 5 & 1,439 & 1,593 \\
\hline 6 & 1,993 & 2,171 \\
\hline 7 & 2,611 & 2,809 \\
\hline 8 & 3,281 & 3,498 \\
\hline 9 & 3,996 & 4,230 \\
\hline 10 & 4,747 & 4,997 \\
\hline 11 & 5,528 & 5,791 \\
\hline 12 & 6,331 & 6,607 \\
\hline 13 & 7,152 & 7,439 \\
\hline 14 & 7,985 & 8,281 \\
\hline 15 & 8,825 & 9,130 \\
\hline 16 & 9,669 & 9,982 \\
\hline 17 & 10,514 & 10,833 \\
\hline 18 & 11,357 & 11,680 \\
\hline 19 & 12,196 & 12,523 \\
\hline 20 & 13,029 & 13,359 \\
\hline 21 & 13,857 & 14,188 \\
\hline 22 & 14,678 & 15,009 \\
\hline 23 & 15,493 & 15,822 \\
\hline 24 & 16,303 & 16,630 \\
\hline 25 & 17,110 & 17,431 \\
\hline & & \\
\hline
\end{tabular}

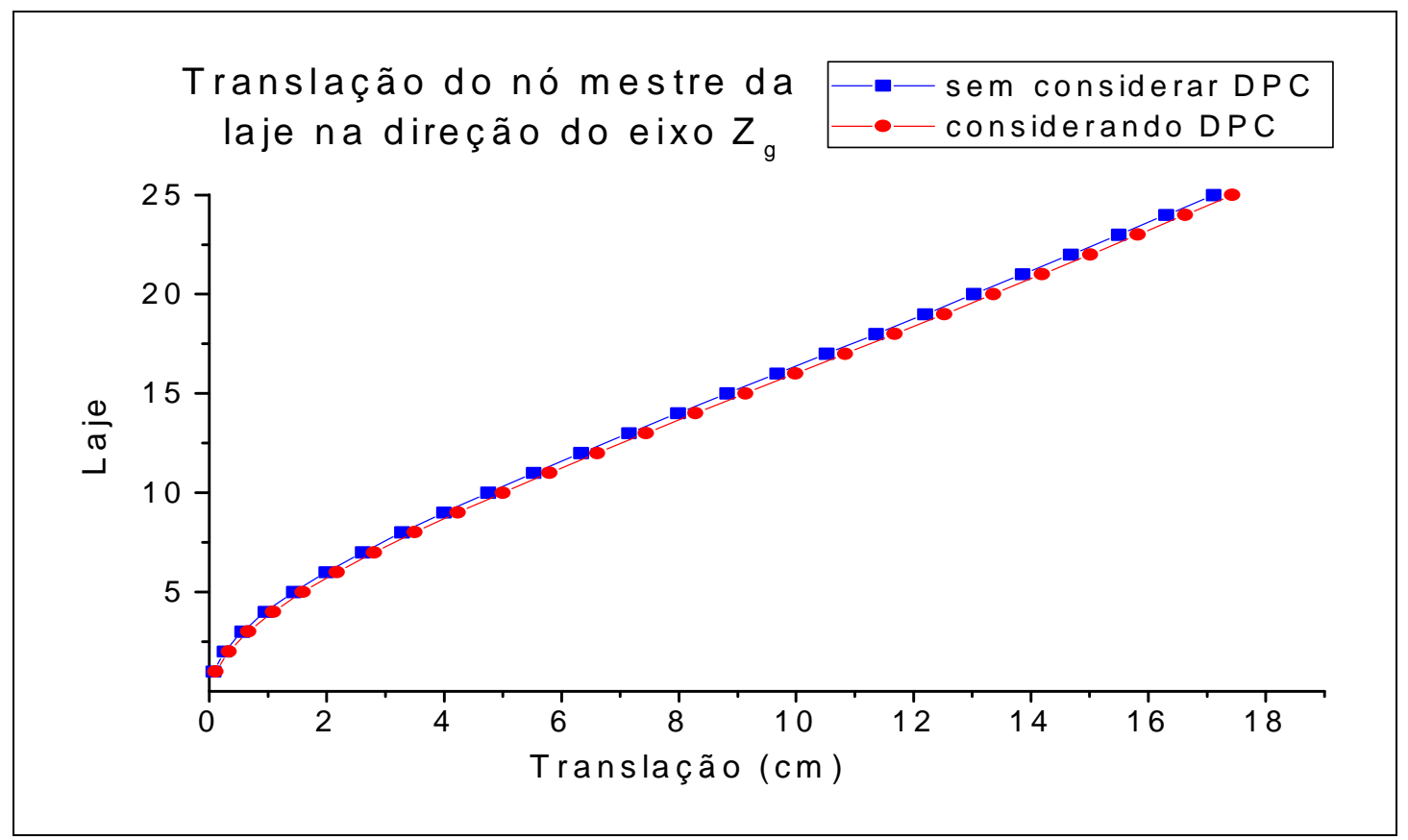

Figura 50 - Translação do nó mestre da laje na direção do eixo $Z_{\mathrm{g}}$ 
Tabela 28 - Rotação das lajes em torno do eixo $\mathrm{X}_{\mathrm{g}}\left(10^{-3} \mathrm{rad}\right)$

\begin{tabular}{|c|c|c|}
\hline Laje & sem considerar DPC & considerando DPC \\
\hline 1 & 0,01206 & 0,02183 \\
\hline 2 & 0,04391 & 0,06324 \\
\hline 3 & 0,09018 & 0,1158 \\
\hline 4 & 0,1467 & 0,1764 \\
\hline 5 & 0,2101 & 0,2425 \\
\hline 6 & 0,2780 & 0,3120 \\
\hline 7 & 0,3484 & 0,3833 \\
\hline 8 & 0,4196 & 0,4551 \\
\hline 9 & 0,4906 & 0,5262 \\
\hline 10 & 0,5602 & 0,5959 \\
\hline 11 & 0,6278 & 0,6636 \\
\hline 12 & 0,6929 & 0,7285 \\
\hline 13 & 0,7549 & 0,7905 \\
\hline 14 & 0,8135 & 0,8490 \\
\hline 15 & 0,8685 & 0,9040 \\
\hline 16 & 0,9198 & 0,9552 \\
\hline 17 & 0,9672 & 1,003 \\
\hline 18 & 1,011 & 1,046 \\
\hline 19 & 1,051 & 1,086 \\
\hline 20 & 1,087 & 1,122 \\
\hline 21 & 1,119 & 1,155 \\
\hline 22 & 1,149 & 1,184 \\
\hline 23 & 1,176 & 1,211 \\
\hline 24 & 1,201 & 1,236 \\
\hline 25 & 1,225 & 1,258 \\
\hline & & \\
\hline
\end{tabular}

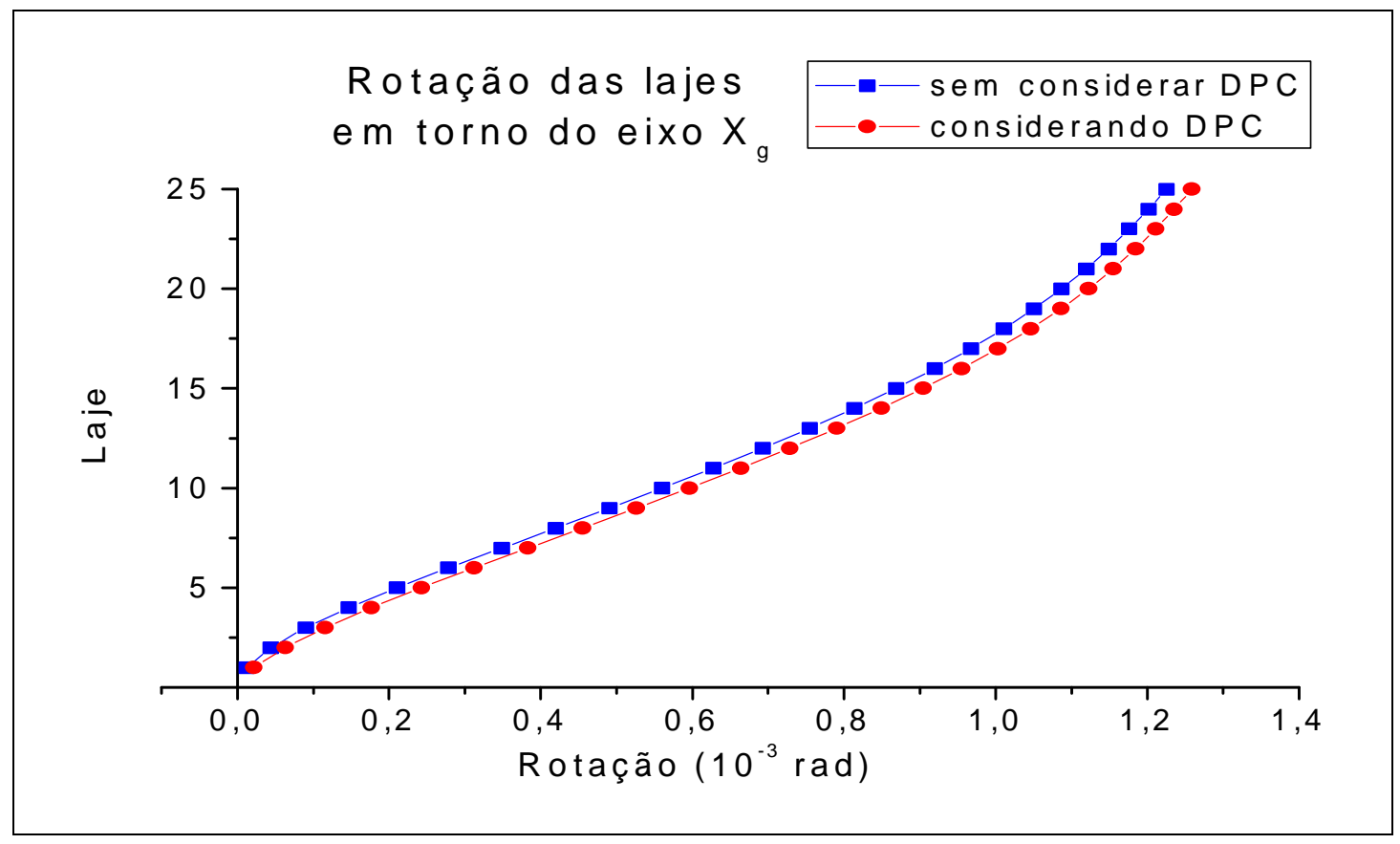

Figura 51 - Rotação das lajes em torno do eixo $X_{g}$ 
Tabela 29 - Soma dos esforços cortantes nos núcleos $\mathrm{N}_{1}$ e $\mathrm{N}_{2}$ na direção do eixo $\mathrm{Z}_{\mathrm{g}}$

\begin{tabular}{|c|c|c|c|c|}
\hline \multirow[b]{2}{*}{ Andar } & \multicolumn{2}{|c|}{ sem considerar DPC } & \multicolumn{2}{|c|}{ considerando DPC } \\
\hline & $\begin{array}{c}\text { Cortante } \\
(\mathrm{kN})\end{array}$ & $\begin{array}{l}\% \text { esforço cor- } \\
\text { tante no andar }\end{array}$ & $\begin{array}{c}\text { Cortante } \\
(\mathrm{kN})\end{array}$ & $\begin{array}{l}\% \text { esforço cor- } \\
\text { tante no andar }\end{array}$ \\
\hline 1 & 2506,98 & 96,92 & 2293,82 & 88,68 \\
\hline 2 & 2319,67 & 92,57 & 2306,86 & 92,05 \\
\hline 3 & 2143,29 & 88,67 & 2106,22 & 87,14 \\
\hline 4 & 1977,35 & 85,12 & 1950,57 & 83,96 \\
\hline 5 & 1820,45 & 81,79 & 1798,06 & 80,79 \\
\hline 6 & 1673,02 & 78,71 & 1654,73 & 77,84 \\
\hline 7 & 1534,11 & 75,81 & 1518,76 & 75,05 \\
\hline 8 & 1401,28 & 72,99 & 1389,54 & 72,38 \\
\hline 9 & 1275,35 & 70,29 & 1266,25 & 69,78 \\
\hline 10 & 1155,85 & 67,67 & 1148,19 & 67,22 \\
\hline 11 & 1040,63 & 65,01 & 1034,74 & 64,64 \\
\hline 12 & 929,48 & 62,27 & 925,26 & 61,99 \\
\hline 13 & 821,89 & 59,39 & 819,16 & 59,19 \\
\hline 14 & 717,92 & 56,33 & 716,00 & 56,18 \\
\hline 15 & 616,25 & 52,91 & 615,21 & 52,82 \\
\hline 16 & 516,20 & 48,96 & 516,30 & 48,97 \\
\hline 17 & 417,80 & 44,26 & 418,81 & 44,37 \\
\hline 18 & 320,32 & 38,45 & 322,28 & 38,68 \\
\hline 19 & 223,52 & 30,95 & 226,25 & 31,33 \\
\hline 20 & 126,55 & 20,71 & 130,25 & 21,31 \\
\hline 21 & 28,52 & 5,70 & 33,72 & 6,74 \\
\hline 22 & $-67,64$ & - & $-63,32$ & - \\
\hline 23 & $-178,21$ & - & $-165,58$ & - \\
\hline 24 & $-234,45$ & - & $-242,42$ & - \\
\hline 25 & $-556,45$ & - & $-521,68$ & - \\
\hline
\end{tabular}


Tabela 30 - Soma dos esforços cortantes em todos os pilares na direção do eixo $\mathrm{Z}_{\mathrm{g}}$

\begin{tabular}{|c|c|c|c|c|}
\hline \multirow{2}{*}{ Andar } & \multicolumn{2}{|c|}{ sem considerar DPC } & \multicolumn{2}{c|}{ considerando DPC } \\
\cline { 2 - 5 } & $\begin{array}{c}\text { Cortante } \\
(\mathrm{kN})\end{array}$ & $\begin{array}{c}\text { \% esforço cor- } \\
\text { tante no andar }\end{array}$ & $\begin{array}{c}\text { Cortante } \\
(\mathrm{kN})\end{array}$ & $\begin{array}{c}\text { \% esforço cor- } \\
\text { tante no andar }\end{array}$ \\
\hline 1 & 79,70 & 3,08 & 292,86 & 11,32 \\
\hline 2 & 186,31 & 7,43 & 199,12 & 7,95 \\
\hline 3 & 273,74 & 11,33 & 310,81 & 12,86 \\
\hline 4 & 345,77 & 14,88 & 372,55 & 16,04 \\
\hline 5 & 405,27 & 18,21 & 427,66 & 19,21 \\
\hline 6 & 452,65 & 21,29 & 470,94 & 22,16 \\
\hline 7 & 489,43 & 24,19 & 504,78 & 24,95 \\
\hline 8 & 518,44 & 27,01 & 530,18 & 27,62 \\
\hline 9 & 539,15 & 29,71 & 548,25 & 30,22 \\
\hline 10 & 552,29 & 32,33 & 559,95 & 32,78 \\
\hline 11 & 560,17 & 34,99 & 566,06 & 35,36 \\
\hline 12 & 563,18 & 37,73 & 567,40 & 38,01 \\
\hline 13 & 561,96 & 40,61 & 564,69 & 40,81 \\
\hline 14 & 556,55 & 43,67 & 558,47 & 43,82 \\
\hline 15 & 548,38 & 47,09 & 549,42 & 47,18 \\
\hline 16 & 538,20 & 51,04 & 538,10 & 51,03 \\
\hline 17 & 526,08 & 55,74 & 525,07 & 55,63 \\
\hline 18 & 512,79 & 61,55 & 510,83 & 61,32 \\
\hline 19 & 498,65 & 69,05 & 495,92 & 68,67 \\
\hline 20 & 484,55 & 79,29 & 480,85 & 78,69 \\
\hline 21 & 471,43 & 94,30 & 466,23 & 93,26 \\
\hline 22 & 456,42 & - & 452,10 & - \\
\hline 23 & 455,83 & - & 443,20 & - \\
\hline 24 & 400,95 & - & 408,92 & - \\
\hline 25 & 611,91 & - & 577,14 & - \\
\hline & & & & \\
\hline 19 & & & \\
\hline
\end{tabular}


Tabela 31 - Esforço cortante $(\mathrm{kN})$ na direção do eixo $\mathrm{Z}_{\mathrm{g}}$ no pilar 20

\begin{tabular}{|c|c|c|}
\hline Andar & sem considerar DPC & considerando DPC \\
\hline 1 & 6,8336 & 31,057 \\
\hline 2 & 13,689 & 14,279 \\
\hline 3 & 18,703 & 21,758 \\
\hline 4 & 22,423 & 24,317 \\
\hline 5 & 25,058 & 26,580 \\
\hline 6 & 26,823 & 27,967 \\
\hline 7 & 27,882 & 28,756 \\
\hline 8 & 28,371 & 29,034 \\
\hline 9 & 28,397 & 28,900 \\
\hline 10 & 28,050 & 28,429 \\
\hline 11 & 27,404 & 27,686 \\
\hline 12 & 26,518 & 26,726 \\
\hline 13 & 25,444 & 25,592 \\
\hline 14 & 24,225 & 24,327 \\
\hline 15 & 22,901 & 22,962 \\
\hline 16 & 21,503 & 21,530 \\
\hline 17 & 20,065 & 20,059 \\
\hline 18 & 18,617 & 18,578 \\
\hline 19 & 17,118 & 17,112 \\
\hline 20 & 15,813 & 15,699 \\
\hline 21 & 14,521 & 14,335 \\
\hline 22 & 13,369 & 13,198 \\
\hline 23 & 12,374 & 11,785 \\
\hline 24 & 11,358 & 11,825 \\
\hline 25 & 13,619 & 11,505 \\
\hline & & \\
\hline
\end{tabular}

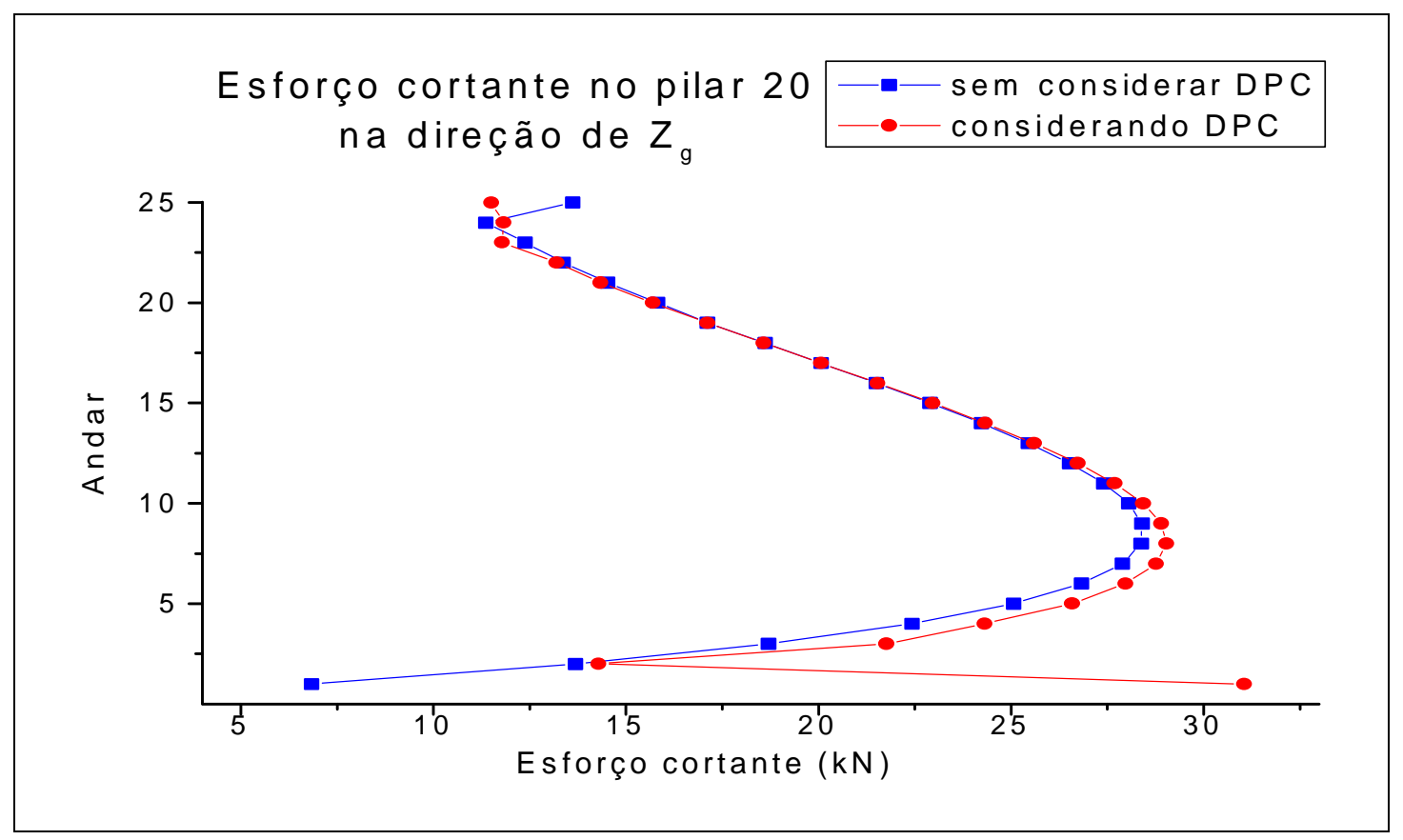

Figura 52 - Esforço cortante no pilar 20 na direção de $Z_{g}$ 
Tabela 32 - Momento fletor (kN.cm) em torno do eixo y local do pilar 7 (paralelo a $\mathrm{Y}_{\mathrm{g}}$ ), na extremidade inferior do elemento.

\begin{tabular}{|c|c|c|}
\hline Andar & sem considerar DPC & considerando DPC \\
\hline 1 & $5.580,7$ & $9.808,9$ \\
\hline 2 & $5.755,6$ & $5.095,5$ \\
\hline 3 & $5.870,9$ & $6.014,3$ \\
\hline 4 & $5.949,8$ & $5.951,4$ \\
\hline 5 & $5.991,5$ & $6.010,9$ \\
\hline 6 & $6.001,2$ & $6.011,8$ \\
\hline 7 & $5.982,4$ & $5.989,0$ \\
\hline 8 & $5.938,2$ & $5.940,5$ \\
\hline 9 & $5.872,3$ & $5.870,5$ \\
\hline 10 & $5.788,3$ & $5.782,2$ \\
\hline 11 & $5.689,0$ & $5.678,8$ \\
\hline 12 & $5.577,8$ & $5.563,4$ \\
\hline 13 & $5.458,0$ & $5.439,3$ \\
\hline 14 & $5.332,8$ & $5.309,7$ \\
\hline 15 & $5.205,4$ & $5.177,5$ \\
\hline 16 & $5.078,9$ & $5.046,0$ \\
\hline 17 & $4.956,8$ & $4.918,5$ \\
\hline 18 & $4.842,1$ & $4.798,1$ \\
\hline 19 & $4.739,2$ & $4.688,3$ \\
\hline 20 & $4.648,6$ & $4.592,1$ \\
\hline 21 & $4.584,3$ & $4.514,6$ \\
\hline 22 & $4.510,6$ & $4.451,6$ \\
\hline 23 & $4.585,5$ & $4.447,5$ \\
\hline 24 & $4.210,9$ & $4.286,6$ \\
\hline 25 & $5.744,9$ & $5.207,5$ \\
\hline & & \\
\hline
\end{tabular}

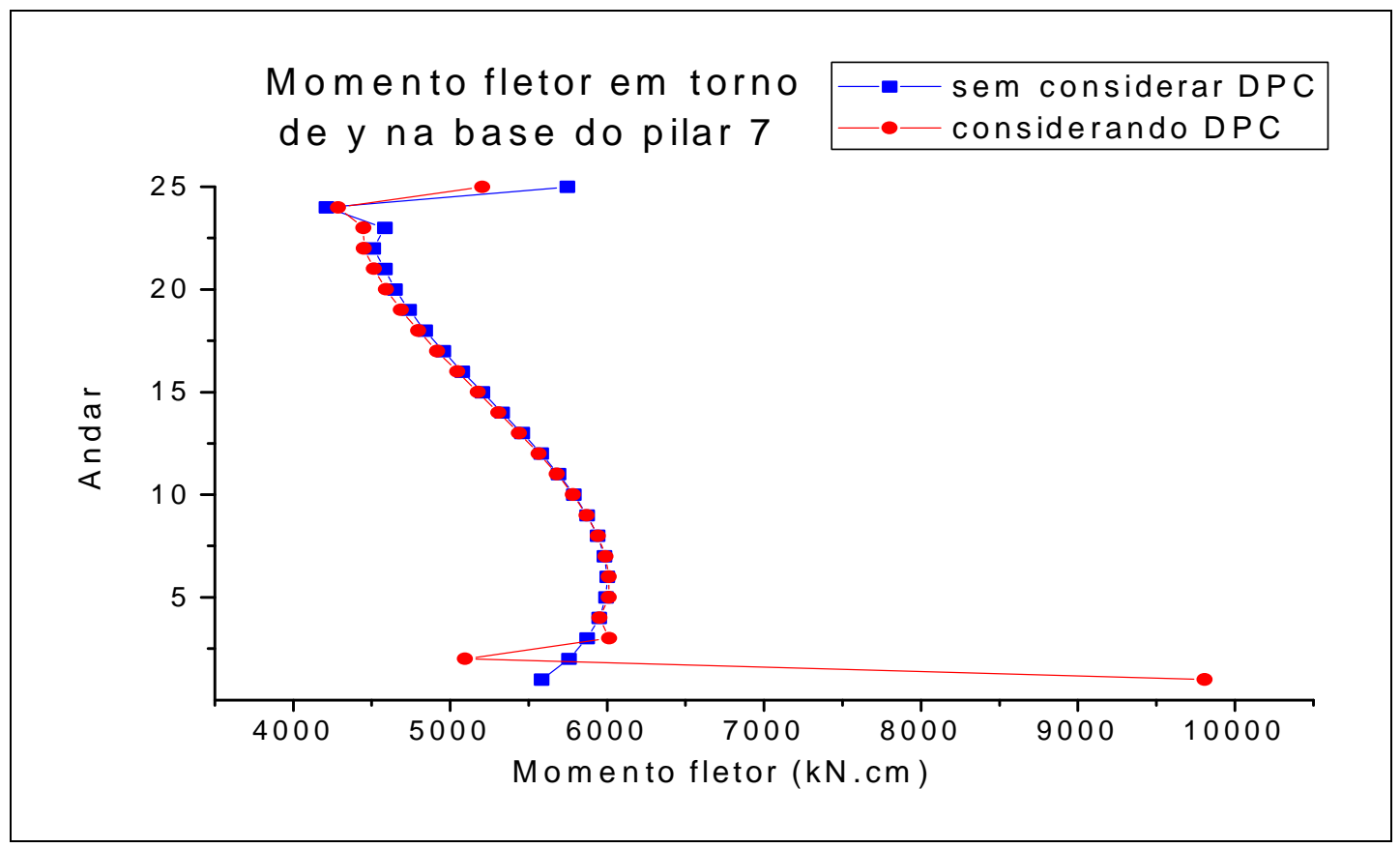

Figura 53 - Momento fletor em torno de y na base do pilar 7 
Tabela 33 - Momento torçor (kN.cm) no núcleo $\mathrm{N}_{1}$.

\begin{tabular}{|c|c|c|}
\hline Andar & sem considerar DPC & considerando DPC \\
\hline 1 & $3.954,06$ & $26.611,39$ \\
\hline 2 & $3.490,45$ & 606,01 \\
\hline 3 & $3.116,72$ & $3.139,80$ \\
\hline 4 & $2.808,10$ & $2.449,58$ \\
\hline 5 & $2.556,27$ & $2.376,73$ \\
\hline 6 & $2.340,85$ & $2.187,97$ \\
\hline 7 & $2.152,72$ & $2.048,92$ \\
\hline 8 & $1.992,49$ & $1.912,61$ \\
\hline 9 & $1.842,24$ & $1.787,84$ \\
\hline 10 & $1.706,49$ & $1.666,58$ \\
\hline 11 & $1.579,19$ & $1.552,24$ \\
\hline 12 & $1.459,04$ & $1.439,45$ \\
\hline 13 & $1.343,41$ & $1.327,99$ \\
\hline 14 & $1.227,65$ & $1.221,40$ \\
\hline 15 & $1.116,63$ & $1.113,34$ \\
\hline 16 & $1.004,46$ & $1.006,02$ \\
\hline 17 & 893,18 & 898,22 \\
\hline 18 & 781,33 & 790,02 \\
\hline 19 & 666,39 & 680,69 \\
\hline 20 & 549,55 & 570,08 \\
\hline 21 & 428,84 & 453,88 \\
\hline 22 & 302,20 & 349,00 \\
\hline 23 & 164,82 & 176,46 \\
\hline 24 & 305,86 & 271,34 \\
\hline 25 & $-183,86$ & $-589,34$ \\
\hline
\end{tabular}

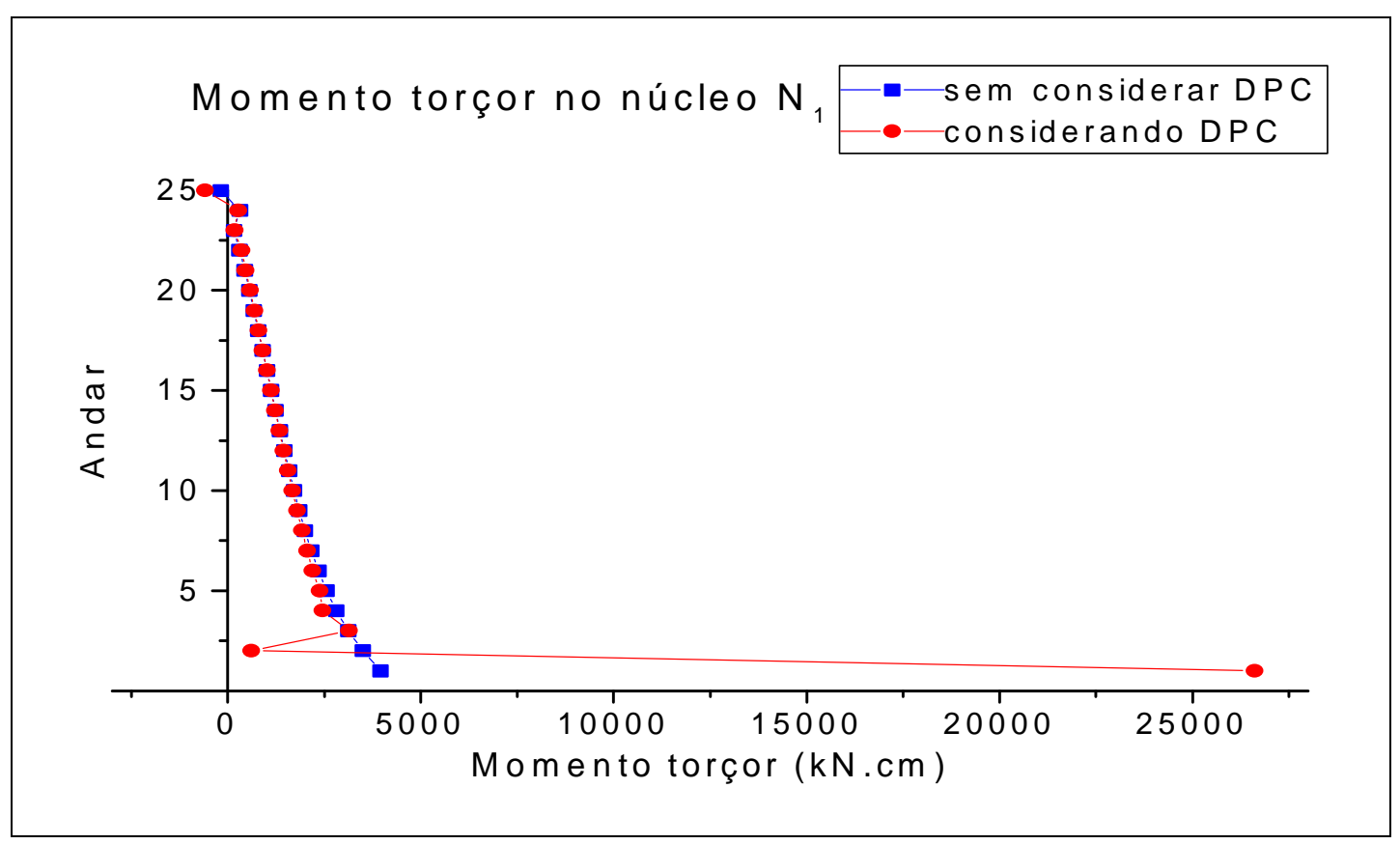

Figura 54 - Momento torçor no núcleo $\mathrm{N}_{1}$ 
Este exemplo reforça algumas das principais alterações que se pode notar ao se considerar o efeito da deformação por esforço cortante nos elementos verticais de contraventamento de uma estrutura, resumidas a seguir:

- A translação do nó mestre das lajes na direção do carregamento é sempre aumentada.

- É bastante freqüiente a ocorrência de redução de rigidez da estrutura à torção e o conseqüente aumento na rotação das lajes, o que geralmente acarreta acréscimo do momento torçor nos núcleos resistentes. Neste exemplo o momento torçor no núcleo $\mathrm{N}_{1}$, no primeiro pavimento, teve um incremento de $573,01 \%$, passando de $3.954,06 \mathrm{kN} . \mathrm{cm}$ para $26.611,39 \mathrm{kN} . \mathrm{cm}$, como pode ser visto na tabela 33 .

- Ocorre uma redistribuição do esforço cortante entre núcleos resistentes e pilares, sendo que os últimos passam a absorver maior esforço. Este aumento no esforço cortante nos pilares é, algumas vezes, bastante significativo. Neste exemplo, a somatória do esforço cortante absorvido pelos pilares segundo a direção do eixo $\mathrm{Z}_{\mathrm{g}}$ teve um aumento, no primeiro pavimento, de $267,45 \%$, passando de $79,70 \mathrm{kN}$ para $292,86 \mathrm{kN}$.

- De um modo geral, as alterações, seja nos deslocamentos ou nos esforços, são mais notáveis nos pavimentos mais baixos, especialmente no primeiro andar 


\section{7 - FATORES DE FORMA DE PERFIS ESTRUTURAIS DE AÇO FORMADOS A FRIO}

O processo de cálculo dos fatores de forma, conforme descrito no capítulo 4 , permitiu o desenvolvimento de uma sub-rotina que foi incorporada ao programa CEASO 01. Com isso, pôde-se calcular os fatores de forma dos núcleos resistentes e realizar a análise estrutural dos cinco exemplos do capítulo 6. Essa sub-rotina, entretanto, possibilita o cálculo dos fatores de forma de qualquer seção transversal aberta de paredes delgadas, como é o caso dos perfis de aço formados a frio.

Este capítulo tem por objetivo oferecer à análise de estruturas metálicas valores de fatores de forma obtidos por um processo de cálculo mais refinado do que os usualmente empregados, além de demonstrar sua aplicabilidade por meio de dois exemplos.

\subsection{Tabelas com o valor dos fatores de forma dos perfis formados a frio}

São apresentadas a seguir tabelas com as características geométricas das seções transversais dos seguintes perfis formados a frio, conforme a NBR 6355 da ABNT (1980).

Nas tabelas, as grandezas apresentadas têm o seguinte valor:

$\mathrm{h}, \mathrm{B}, \mathrm{d}, \mathrm{e}, \mathrm{r}, \mathrm{e}_{\mathrm{y}}$ : indicadas na figura referente a cada perfil.

$\mathrm{S}, \mathrm{M}, \mathrm{J}_{\mathrm{x}}, \mathrm{J}_{\mathrm{y}}, \mathrm{J}_{\mathrm{xy}}, \mathrm{i}_{\mathrm{x}}, \mathrm{i}_{\mathrm{y}}$ : respectivamente, a área da seção transversal, o peso por unidade de comprimento, os momentos de inércia em relação aos eixos x e y, o produto de inércia e os raios de giração em relação aos eixos $\mathrm{x}$ e $\mathrm{y}$, dados por $i_{x}=\sqrt{\frac{J_{x}}{S}}$ e $i_{y}=\sqrt{\frac{J_{y}}{S}}$ 
$\mathrm{W}_{\mathrm{x}}, \mathrm{W}_{\mathrm{y}}$ : módulo resistente de cada seção.

$\mathrm{J}_{\mathrm{xo}}, \mathrm{J}_{\text {yo }}, \mathrm{i}_{\text {máx }}, \mathrm{i}_{\text {mín }}, \alpha$ : respectivamente os momentos principais de inércia, os raios de giração correspondentes a eles e o ângulo entre o sistema de eixos principais $\left(\mathrm{x}_{\mathrm{O}}, \mathrm{y}_{\mathrm{O}}\right)$ e o sistema $\mathrm{xy}$.

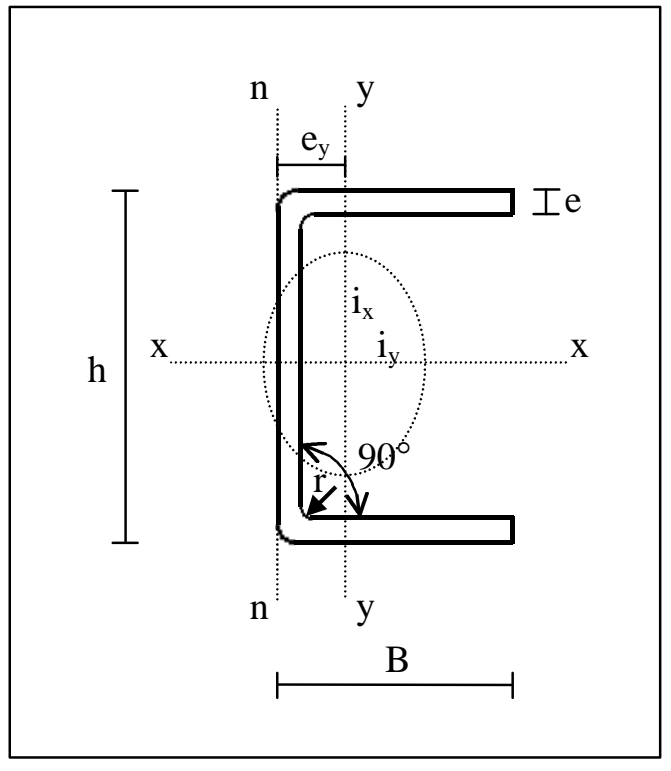

Figura 55 - Perfil U simples

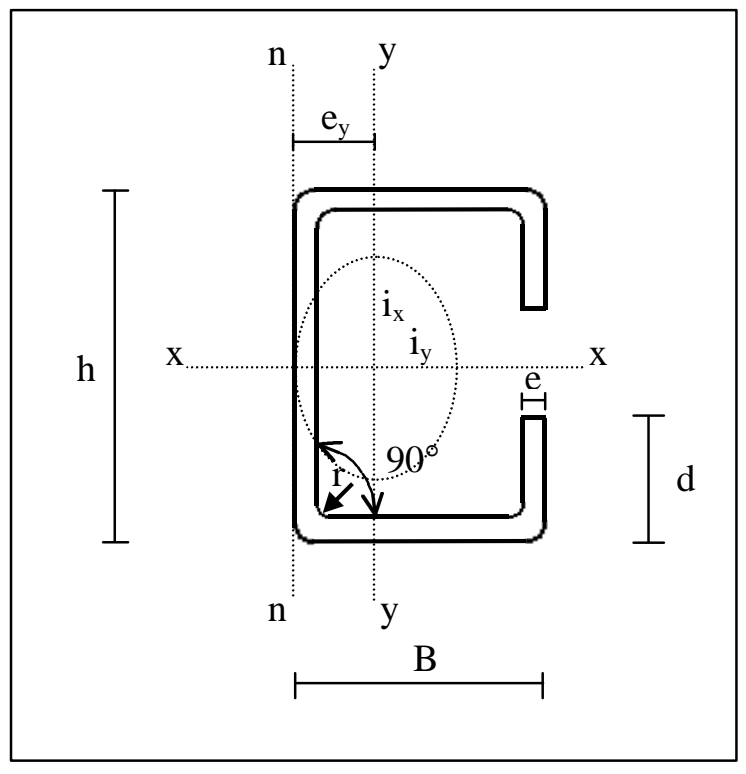

Figura 56 - Perfil U enrijecido

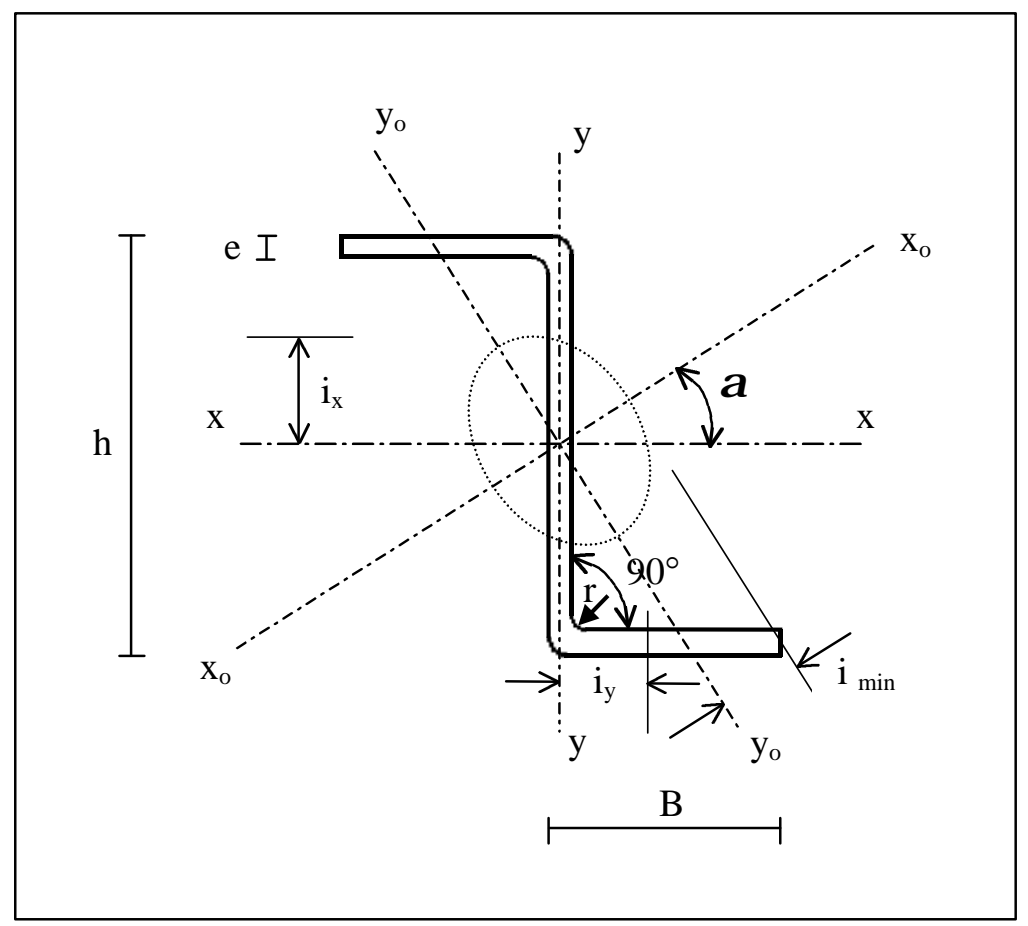

Figura 57 - Perfil Z simples 


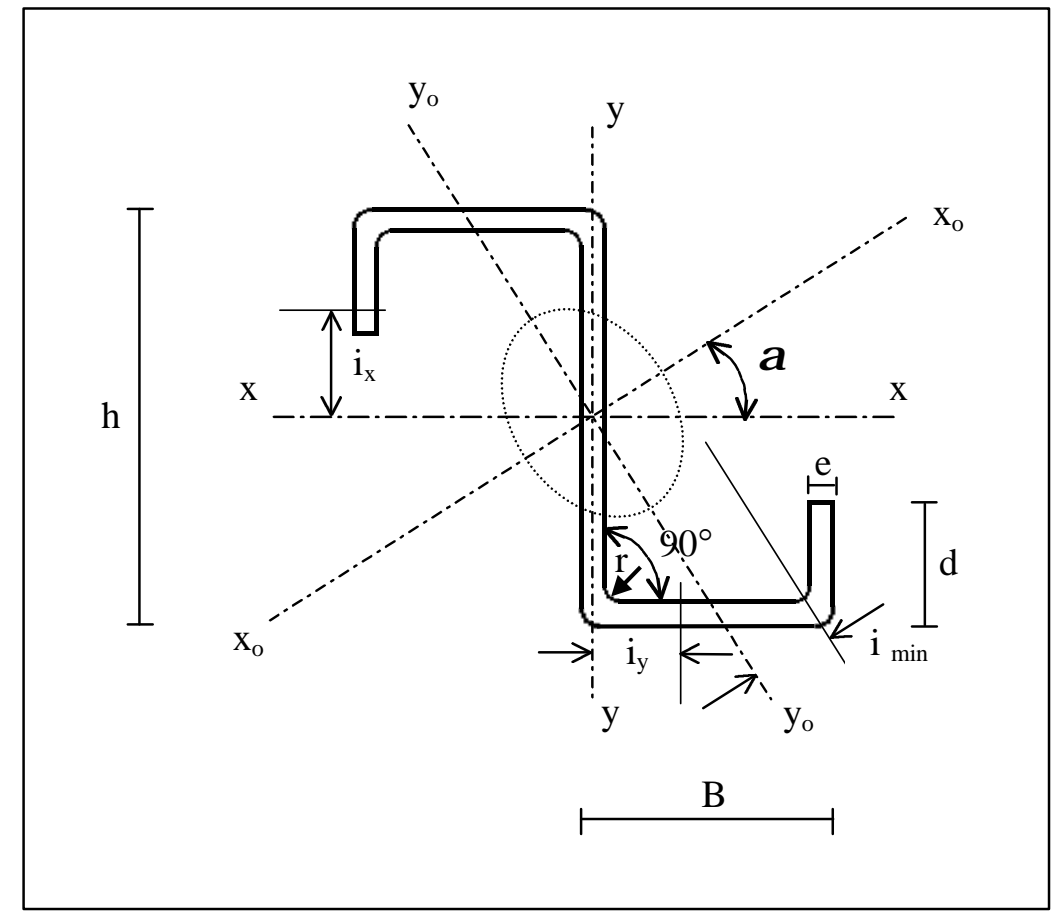

Figura 58 - Perfil Z enrijecido

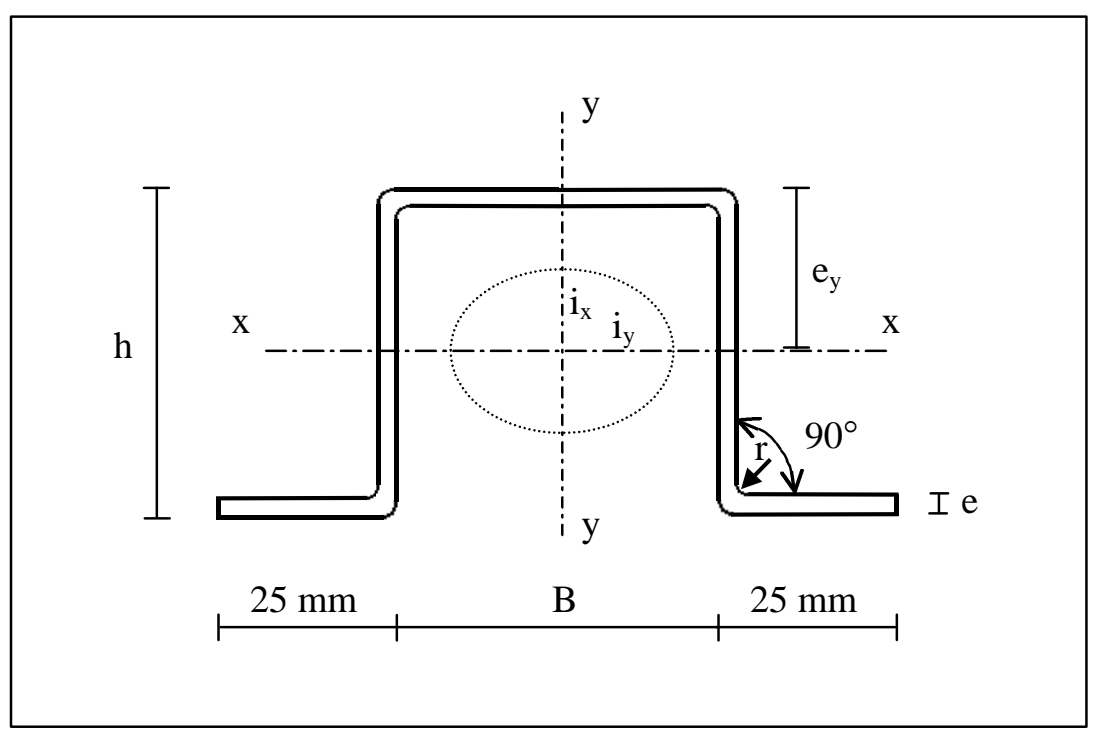

Figura 59 - Perfil cartola

Nas figuras 55, 56, 57, 58 e 59 são apresentados os cinco tipos de perfis formados a frio estabelecidos na NBR 6355/80, com as dimensões que os caracterizam. Em todos eles, $r=$ e. Para perfis U simples, Z simples e cartola há um conjunto de dimensões $h$ e $B$ às quais correspondem várias espessuras (e), enquanto para os perfis $U$ enrijecidos e $Z$ enrijecidos há um conjunto de dimensões 
$\mathrm{h}, \mathrm{B}$ e $\mathrm{d}$ às quais correspondem várias espessuras. As tabelas a seguir apresentam apenas a maior e a menor espessura para cada conjunto de dimensões. 
Tabela 34 - Características geométricas da seção transversal dos perfis U simples

\begin{tabular}{|c|c|c|c|c|c|c|c|c|c|c|c|c|c|c|}
\hline $\mathrm{h}$ & B & $e=r$ & S & $\mathrm{M}$ & $\mathrm{J}_{\mathrm{x}}$ & $\mathrm{W}_{\mathrm{x}}$ & $i_{x}$ & $e_{y}$ & $\mathrm{~J}_{\mathrm{y}}$ & $\mathrm{W}_{\mathrm{y}}$ & $\mathrm{i}_{\mathrm{y}}$ & \multirow[b]{2}{*}{$\alpha_{x x}$} & \multirow[b]{2}{*}{$\alpha_{x y}$} & \multirow[b]{2}{*}{$\alpha_{y y}$} \\
\hline $\mathrm{mm}$ & $\mathrm{mm}$ & $\mathrm{mm}$ & $\mathrm{cm}^{2}$ & $\mathrm{~kg} / \mathrm{m}$ & $\mathrm{cm}^{4}$ & $\mathrm{~cm}^{3}$ & $\mathrm{~cm}$ & $\mathrm{~cm}$ & $\mathrm{~cm}^{4}$ & $\mathrm{~cm}^{3}$ & $\mathrm{~cm}$ & & & \\
\hline \multirow[t]{2}{*}{200} & \multirow[t]{2}{*}{50} & 4,76 & 13,39 & 10,51 & 686,2 & 68,6 & 7,15 & 1,04 & 25,76 & 6,51 & 1,38 & 7,2920 & 0,0 & 1,6187 \\
\hline & & 1,90 & 5,55 & 4,36 & 299,3 & 29,9 & 7,33 & 0,91 & 11,20 & 2,74 & 1,41 & 7,2133 & 0,0 & 1,6286 \\
\hline \multirow[t]{2}{*}{150} & \multirow[t]{2}{*}{50} & 4,76 & 11,01 & 8,64 & 338,0 & 45,0 & 5,54 & 1,21 & 23,84 & 6,30 & 1,47 & 5,0195 & 0,0 & 1,8482 \\
\hline & & 1,90 & 4,60 & 3,61 & 149,9 & 19,9 & 5,70 & 1,08 & 10,42 & 2,65 & 1,50 & 5,0003 & 0,0 & 1,8586 \\
\hline \multirow[t]{2}{*}{127} & \multirow[t]{2}{*}{50} & 4,76 & 9,91 & 7,78 & 255,9 & 35,5 & 4,77 & 1,32 & 22,66 & 6,16 & 1,51 & 4,1350 & 0,0 & 2,0325 \\
\hline & & 1,52 & 3,35 & 2,63 & 82,21 & 12,9 & 4,94 & 1,17 & 8,06 & 2,10 & 1,54 & 4,1342 & 0,0 & 2,0427 \\
\hline \multirow[t]{2}{*}{100} & \multirow[t]{2}{*}{50} & 4,76 & 8,63 & 6,77 & 127,5 & 25,4 & 3,84 & 1,48 & 20,39 & 5,94 & 1,55 & 3,2186 & $\overline{0,0}$ & 2,3921 \\
\hline & & 1,52 & 2,94 & 2,31 & 47,32 & 9,4 & 4,00 & 1,32 & 7,49 & 2,04 & 1,59 & 3,2397 & 0,0 & 2,3948 \\
\hline \multirow[t]{2}{*}{100} & \multirow[t]{2}{*}{40} & 4,76 & 7,67 & 6,02 & 105,9 & 21,1 & 3,71 & 1,11 & 11,09 & 3,84 & 1,20 & 4,0534 & 0,0 & 2,0485 \\
\hline & & 1,52 & 2,64 & 2,07 & 39,95 & 7,9 & 3,88 & 0,96 & 4,05 & 1,33 & 1,23 & 4,0652 & 0,0 & 2,0614 \\
\hline \multirow[t]{2}{*}{75} & \multirow[t]{2}{*}{40} & 4,76 & 6,48 & 5,09 & 52,75 & 14,0 & 2,85 & 1,27 & 10,00 & 3,66 & 1,24 & 3,0100 & 0,0 & 2,5135 \\
\hline & & 1,52 & 2,26 & 1,77 & 20,53 & 5,4 & 3,01 & 1,10 & 3,70 & 1,28 & 1,27 & 3,0483 & 0,0 & 2,5132 \\
\hline \multirow[t]{2}{*}{50} & \multirow[t]{2}{*}{25} & 3,04 & 2,67 & 2,10 & 9,55 & 3,8 & 1,88 & 0,77 & 1,59 & 0,92 & 0,77 & 3,2029 & 0,0 & 2,3901 \\
\hline & & 1,52 & 1,42 & 1,12 & 5,51 & 2,2 & 1,96 & 0,70 & 0,88 & 0,49 & 0,78 & 3,2327 & 0,0 & 2,3939 \\
\hline
\end{tabular}


Tabela 35 - Características geométricas da seção transversal dos perfis U enrijecidos

\begin{tabular}{|c|c|c|c|c|c|c|c|c|c|c|c|c|c|c|c|}
\hline $\mathrm{h}$ & B & d & $\mathrm{e}=\mathrm{r}$ & S & M & $\mathrm{J}_{\mathrm{x}}$ & $\mathrm{W}_{\mathrm{x}}$ & $i_{x}$ & $e_{y}$ & $\mathrm{~J}_{\mathrm{y}}$ & $\mathrm{W}_{\mathrm{y}}$ & $\mathrm{i}_{\mathrm{y}}$ & \multirow[b]{2}{*}{$\alpha_{x x}$} & \multirow[b]{2}{*}{$\alpha_{x y}$} & \multirow[b]{2}{*}{$\alpha_{\mathrm{yy}}$} \\
\hline $\mathrm{mm}$ & $\mathrm{mm}$ & $\mathrm{mm}$ & $\mathrm{mm}$ & $\mathrm{cm}^{2}$ & $\mathrm{~kg} / \mathrm{m}$ & $\mathrm{cm}^{4}$ & $\mathrm{~cm}^{3}$ & $\mathrm{~cm}$ & $\mathrm{~cm}$ & $\mathrm{~cm}^{4}$ & $\mathrm{~cm}^{3}$ & $\mathrm{~cm}$ & & & \\
\hline \multirow[t]{2}{*}{300} & \multirow[t]{2}{*}{85} & \multirow[t]{2}{*}{25} & 4,76 & 22,97 & 18,03 & 2899,00 & 193,27 & 11,23 & 2,17 & 190,41 & 30,09 & 2,88 & 5,6581 & 0,0 & 1,9457 \\
\hline & & & 1,90 & 9,60 & 7,53 & 1262,60 & 84,17 & 11,47 & 2,19 & 88,82 & 14,09 & 3,04 & 5,5075 & 0,0 & 1,9823 \\
\hline \multirow[t]{2}{*}{250} & \multirow[t]{2}{*}{85} & \multirow[t]{2}{*}{25} & 4,76 & 20,59 & 16,17 & 1872,56 & 149,80 & 9,54 & 2,40 & 180,43 & 29,56 & 2,96 & 4,6172 & 0,0 & 2,1773 \\
\hline & & & 1,90 & 8,65 & 6,79 & 821,83 & 65,75 & 9,75 & 2,42 & 84,18 & 13,85 & 3,12 & 4,5191 & 0,0 & 2,2220 \\
\hline \multirow[t]{2}{*}{200} & \multirow[t]{2}{*}{75} & \multirow[t]{2}{*}{25} & 4,76 & 17,26 & 13,55 & 1012,80 & 101,28 & 7,66 & 2,30 & 123,17 & 23,67 & 2,67 & 4,2198 & 0,0 & 2,3854 \\
\hline & & & 2,66 & 10,08 & 7,92 & 614,20 & 61,42 & 7,80 & 2,32 & 77,80 & 15,02 & 2,78 & 4,1574 & 0,0 & 2,4271 \\
\hline \multirow[t]{2}{*}{200} & \multirow[t]{2}{*}{75} & \multirow[t]{2}{*}{20} & 2,28 & 8,48 & 6,66 & 520,49 & 52,05 & 7,83 & 2,19 & 62,25 & 11,72 & 2,71 & 4,1005 & 0,0 & 2,3221 \\
\hline & & & 1,90 & 7,13 & 5,60 & 440,46 & 44,05 & 7,86 & 2,19 & 53,20 & 10,02 & 2,73 & 4,0887 & 0,0 & 2,3295 \\
\hline \multirow[t]{2}{*}{150} & \multirow[t]{2}{*}{60} & \multirow[t]{2}{*}{20} & 4,76 & 12,19 & 10,19 & 423,49 & 56,47 & 5,71 & 1,89 & 57,70 & 14,02 & 2,11 & 3,9939 & 0,0 & 2,4790 \\
\hline & & & 1,52 & 4,53 & 3,56 & 159,20 & 21,23 & 5,93 & 1,92 & 23,35 & 5,72 & 2,27 & 3,8925 & 0,0 & 2,5650 \\
\hline \multirow[t]{2}{*}{127} & \multirow[t]{2}{*}{50} & \multirow[t]{2}{*}{17} & 3,42 & 8,01 & 6,29 & 189,39 & 29,83 & 4,86 & 1,58 & 25,20 & 7,36 & 1,77 & 4,0407 & 0,0 & 2,4788 \\
\hline & & & 1,52 & 3,79 & 2,97 & 94,41 & 14,87 & 4,99 & 1,60 & 13,38 & 3,93 & 1,88 & 3,9662 & 0,0 & 2,5383 \\
\hline \multirow[t]{2}{*}{100} & \multirow[t]{2}{*}{50} & \multirow[t]{2}{*}{17} & 3,42 & 7,09 & 5,56 & 107,17 & 21,43 & 3,89 & 1,76 & 23,13 & 7,13 & 1,81 & 3,2684 & 0,0 & 3,0276 \\
\hline & & & 1,52 & 3,38 & 2,65 & 54,16 & 10,83 & 4,01 & 1,78 & 12,32 & 3,83 & 1,91 & 3,2318 & 0,0 & 3,1014 \\
\hline \multirow[t]{2}{*}{75} & \multirow[t]{2}{*}{40} & \multirow[t]{2}{*}{15} & 3,04 & 4,90 & 3,85 & 41,18 & 10,98 & 2,90 & 1,48 & 10,38 & 4,13 & 1,46 & 3,1542 & 0,0 & 3,3026 \\
\hline & & & 1,52 & 2,63 & 2,07 & 23,51 & 6,27 & 2,99 & 1,50 & 6,20 & 2,48 & 1,54 & 3,1253 & 0,0 & 3,3800 \\
\hline \multirow[t]{2}{*}{50} & \multirow[t]{2}{*}{25} & \multirow[t]{2}{*}{10} & 3,04 & 2,92 & 2,30 & 10,04 & 4,01 & 1,85 & 0,91 & 2,15 & 1,35 & 0,86 & 3,3913 & 0,0 & 3,0847 \\
\hline & & & 1,52 & 1,64 & 1,29 & 6,23 & 2,49 & 1,95 & 0,92 & 1,44 & 0,91 & 0,94 & 3,3385 & 0,0 & 3,2066 \\
\hline
\end{tabular}


Tabela 36 - Características geométricas da seção transversal dos perfis Z simples

\begin{tabular}{|c|c|c|c|c|c|c|c|c|c|c|c|c|c|c|c|c|c|c|c|}
\hline $\mathrm{h}$ & & $e=r$ & S & M & $\mathrm{J}_{\mathrm{x}}$ & $\mathrm{W}_{\mathrm{x}}$ & $i_{x}$ & $\mathrm{~J}_{\mathrm{y}}$ & $\mathrm{W}_{\mathrm{y}}$ & $\mathrm{i}_{\mathrm{y}}$ & $\mathbf{J}_{\mathrm{xy}}$ & $\alpha$ & $\mathrm{J}_{\mathrm{xo}}$ & $\mathrm{i}_{\text {máx }}$ & $\mathrm{J}_{\mathrm{yo}}$ & $\mathrm{i}_{\text {mín }}$ & \multirow[b]{2}{*}{$\alpha_{x x}$} & \multirow[b]{2}{*}{$\alpha_{x y}$} & \multirow[b]{2}{*}{$\alpha_{y y}$} \\
\hline $\mathrm{mm}$ & $\mathrm{mm}$ & $\mathrm{mm}$ & $\mathrm{cm}^{2}$ & $\mathrm{~kg} / \mathrm{m}$ & $\mathrm{cm}^{4}$ & $\mathrm{~cm}^{3}$ & $\mathrm{~cm}$ & $\mathrm{~cm}^{4}$ & $\mathrm{~cm}^{3}$ & $\mathrm{~cm}$ & $\mathrm{~cm}^{4}$ & graus & $\mathrm{cm}^{4}$ & $\mathrm{~cm}$ & $\mathrm{~cm}^{4}$ & $\mathrm{~cm}$ & & & \\
\hline \multirow[t]{2}{*}{200} & \multirow[t]{2}{*}{50} & 4,76 & 13,39 & 10,51 & 686,76 & 68,68 & 7,16 & 34,96 & 7,34 & 1,62 & 108,97 & 9,24 & 704,50 & 7,25 & 17,23 & 1,13 & 5,1580 & $-0,0274$ & 1,5866 \\
\hline & & 1,90 & 5,56 & 4,36 & 299,26 & 29,93 & 7,34 & 14,96 & 3,05 & 1,64 & 45,53 & 8,88 & 306,37 & 7,42 & 7,84 & 1,19 & 5,1150 & $-0,0347$ & 1,5949 \\
\hline \multirow[t]{2}{*}{150} & \multirow[t]{2}{*}{50} & 4,76 & 11,01 & 8,64 & 338,59 & 45,15 & 5,55 & 34,92 & 7,33 & 1,78 & 80,94 & 14,03 & 358,82 & 5,71 & 14,69 & 1,16 & 3,6617 & $-0,0976$ & 1,7669 \\
\hline & & 1,90 & 4,61 & 3,62 & 149,90 & 19,99 & 5,70 & 14,95 & 3,05 & 1,80 & 34,04 & 13,38 & 158,00 & 5,85 & 6,86 & 1,22 & 3,6589 & $-0,0999$ & 1,7746 \\
\hline \multirow[t]{2}{*}{127} & \multirow[t]{2}{*}{50} & 4,76 & 9,92 & 7,78 & 226,45 & 35,66 & 4,78 & 34,90 & 7,33 & 1,88 & 68,05 & 17,70 & 248,16 & 5,00 & 13,18 & 1,15 & 3,1013 & $-0,0890$ & 1,8943 \\
\hline & & 1,52 & 3,36 & 2,64 & 82,22 & 12,95 & 4,95 & 12,10 & 2,46 & 1,90 & 23,20 & 16,75 & 89,20 & 5,15 & 5,12 & 1,23 & 3,1107 & $-0,0891$ & $\overline{1,9011}$ \\
\hline \multirow[t]{2}{*}{100} & \multirow[t]{2}{*}{50} & 4,76 & 8,63 & 6,78 & 128,05 & 25,61 & 3,85 & 34,87 & 7,32 & 2,01 & 52,91 & 24,32 & 151,97 & 4,20 & 10,96 & 1,13 & 2,5598 & 0,0053 & 2,0955 \\
\hline & & 1,52 & 2,95 & 2,32 & 47,33 & 9,47 & 4,01 & 12,10 & 2,46 & 2,03 & 18,21 & 22,98 & 55,05 & 4,32 & 4,38 & 1,22 & 2,5824 & 0,0052 & 2,0976 \\
\hline \multirow[t]{2}{*}{100} & \multirow[t]{2}{*}{40} & 4,76 & 7,68 & 6,03 & 106,45 & 21,29 & 3,72 & 17,50 & 4,65 & 1,51 & 33,59 & 18,53 & 117,71 & 3,92 & 6,24 & 0,90 & 3,0440 & $-0,0855$ & 1,9043 \\
\hline & & 1,52 & 2,65 & 2,08 & 39,96 & 7,99 & 3,89 & 6,12 & 1,56 & 1,52 & 11,59 & 17,20 & 43,55 & 4,06 & 2,54 & 0,98 & 3,0673 & $-0,0860$ & 1,9130 \\
\hline \multirow[t]{2}{*}{75} & \multirow[t]{2}{*}{40} & 4,76 & 6,49 & 5,09 & 53,34 & 14,22 & 2,87 & 17,48 & 4,65 & 1,64 & 24,65 & 26,98 & 65,89 & 3,19 & 4,93 & 0,87 & 2,4449 & 0,0504 & 2,1488 \\
\hline & & 1,52 & 2,27 & 1,78 & 20,54 & 5,48 & 3,01 & 6,12 & 1,56 & 1,64 & 8,64 & 25,09 & 24,59 & 3,29 & 2,08 & 0,96 & 2,4836 & 0,0492 & 2,1503 \\
\hline \multirow[t]{2}{*}{50} & \multirow[t]{2}{*}{25} & 3,04 & 2,68 & 2,10 & 9,56 & 3,82 & 1,89 & 2,62 & 1,12 & 0,99 & 4,11 & 24,94 & 11,47 & 2,07 & 0,71 & 0,52 & 2,5427 & 0,0053 & 2,0940 \\
\hline & & 1,52 & 1,43 & 1,12 & 5,52 & 2,21 & 1,96 & 1,44 & 0,60 & 1,01 & 2,19 & 23,56 & 6,47 & 2,13 & 0,49 & 0,58 & 2,5750 & 0,0052 & 2,0969 \\
\hline
\end{tabular}


Tabela 37 - Características geométricas da seção transversal dos perfis Z enrijecidos

\begin{tabular}{|c|c|c|c|c|c|c|c|c|c|c|c|c|c|c|c|c|c|c|c|c|}
\hline $\mathrm{h}$ & B & $\mathrm{a}$ & $=\mathrm{r}$ & S & $\mathrm{M}$ & $\mathrm{J}_{\mathrm{x}}$ & $\mathrm{W}_{\mathrm{x}}$ & $i_{x}$ & $\mathrm{~J}_{\mathrm{y}}$ & $\mathrm{W}_{\mathrm{y}}$ & $\mathrm{I}_{\mathrm{y}}$ & $\mathbf{J}_{\mathrm{xy}}$ & $\alpha$ & $\mathrm{J}_{\mathrm{xo}}$ & $\mathrm{i}_{\text {máx }}$ & $\mathrm{J}_{\mathrm{yo}}$ & $i_{\text {mín }}$ & \multirow[b]{2}{*}{$\alpha_{x x}$} & \multirow[b]{2}{*}{$\alpha_{x y}$} & \multirow[b]{2}{*}{$\alpha_{y y}$} \\
\hline $\mathrm{mm}$ & $\mathrm{m}$ & $\mathrm{nm}$ & $\mathrm{nm}$ & $\mathrm{m}^{2}$ & $\mathrm{~g} / \mathrm{m}$ & $\mathrm{cm}^{4}$ & $\mathrm{~cm}^{3}$ & $\mathrm{~cm}$ & $\mathrm{~cm}^{4}$ & $\mathrm{~cm}^{3}$ & $\mathrm{Cm}$ & $\mathrm{cm}^{4}$ & grau & $\mathrm{cm}^{4}$ & $\mathrm{~cm}$ & $\mathrm{~cm}^{4}$ & $\mathrm{~cm}$ & & & \\
\hline \multirow[t]{2}{*}{300} & & \multirow[t]{2}{*}{25} & 76 & 2,9 &, 03 & 899,0 & 93,2 & 1,2 & 76,2 & 3,4 &, 4 & 32,9 & 2,8 & 43,7 & 1,5 & 131,5 & 3 & & 0,3154 & 858 \\
\hline & & & 90 & 60 & 53 & 262,6 & 4,17 & & 31,1 & 5,6 & ,7 & 90,9 & 3,6 & 0 & 1,7 & 0,69 & ,5 & & & 8 \\
\hline \multirow[t]{2}{*}{250} & \multirow[t]{2}{*}{85} & \multirow[t]{2}{*}{25} & 4,76 & 20,5 & 5,17 & 372,5 & 49,8 & 54 & 276,2 & 3,4 & 6 & 21,8 & 6,5 & 028,0 &, 92 & 20,7 & 2,4 & &, 3202 &, 0243 \\
\hline & & & 1,90 & 8,65 &,, 79 & 21,83 & 5,75 &, 75 & 131,1 & 5,6 & 3,8 & 240,5 & 17,4 & 97,34 & 10,1 & 50,59 & 2,5 & 4206 & 0,3273 & נט, \\
\hline \multirow[t]{2}{*}{200} & \multirow[t]{2}{*}{75} & \multirow[t]{2}{*}{25} & 76 & 17,2 & 3,55 & 12,8 & 1,2 & ,66 & 96,3 & 7,0 & 3,3 & 26,9 & 9,3 & 127,5 & 8,08 & 1,52 &, 1 & & & \\
\hline & & & 2,66 & 10,0 & 7,92 & 614,20 & 61,42 & $\overline{7,80}$ & 125,9 & 17,1 & 3,5 & 206,1 & 20,0 & 889,63 & 8,27 & 50,55 & 2,2 &, 1632 & $-0,3539$ & 2,1949 \\
\hline \multirow[t]{2}{*}{200} & \multirow[t]{2}{*}{75} & \multirow[t]{2}{*}{20} & 28 & 48 & ,66 & 520,49 & 2,05 & 83 & 3,68 & 3,3 & 3,4 & 167,5 & 2 & 78,96 &, 26 & 0,21 & & & 716 & 112 \\
\hline & & & 1,90 & 7,13 & 5,60 & 440,46 & 44,05 & 7,86 & 84,53 & 11,4 & 3,4 & 142,7 & 19,3 & 90,65 & 8,30 & 34,33 & 2,1 & & 0,2721 & 2,1170 \\
\hline \multirow[t]{2}{*}{150} & \multirow[t]{2}{*}{60} & \multirow[t]{2}{*}{20} & 4,76 & 2,1 & 0,19 & 423,49 & 56,47 &, 71 & 92,87 & 16,1 & 2,6 & 144,8 & 20,6 & 777,95 & 6,07 & 38,42 & 1,7 & 485 & 0,3288 & 2,2227 \\
\hline & & & 1,52 & 53 & 3,56 & 159,20 & 21,23 & 93 & 38,77 & 6,54 & 2,9 & 58,70 & 22,1 & 83,08 & 6,36 & 14,90 & 1,8 & 9843 & 0,3295 & 2,275 \\
\hline \multirow[t]{2}{*}{127} & \multirow[t]{2}{*}{50} & \multirow[t]{2}{*}{17} & 3,42 & 8,01 & 6,29 & 189,39 & 29,83 & 4,86 & 40,97 & 8,49 & 2,2 & 65,05 & 20,6 & 213,87 & 5,17 & 16,50 & 1,4 & 3,0795 & $-0,3446$ & 2,2258 \\
\hline & & & 1,52 & 3,79 & 2,97 & 94,41 & 14,87 & ,99 & 22,12 & 4,49 & 2,4 & 34,08 & 21,6 & 107,94 & 5,3 & 8,59 & 1,5 & 0316 & 0,3453 & 2,262 \\
\hline \multirow[t]{2}{*}{100} & \multirow[t]{2}{*}{0} & \multirow[t]{2}{*}{17} & 3,42 & 7,09 & 5,56 & 107,17 & 21,43 & 3,89 & 40,97 & 8,48 & 2,4 & 49,86 & 28,2 & 133,92 & 4,35 & 14,22 & 1,4 & 5861 & $-0,1937$ & 2,4922 \\
\hline & & & 1,52 & 3,38 & 2,65 & 54,16 & 10,83 & 4,01 & 22,12 & 4,49 & 2,5 & 26,31 & 29,3 & 68,94 & 4,52 & 7,34 & 1,4 & 680 & 0,1843 & 2,5256 \\
\hline \multirow[t]{2}{*}{75} & \multirow[t]{2}{*}{40} & \multirow[t]{2}{*}{1} & 3,04 & 4,90 & 3,85 & 41,18 & 10,98 & 2,90 & 19,07 & 4,96 & 1,9 & 21,05 & 31,1 & 53,90 & 3,32 & 6,35 & 1,1 & 2,5062 & $-0,1776$ & 2,6198 \\
\hline & & & 1,52 & 2,63 & 2,07 & 3,51 & 6,27 & 2,99 & 11,54 & 2,94 & 2,0 & 12,53 & 32,2 & ,40 & 3,45 & 3,64 & 1,1 & 1954 & 0,1650 & 2,6502 \\
\hline \multirow[t]{2}{*}{50} & \multirow[t]{2}{*}{25} & 10 & 3,04 & 2,92 & 2,30 & 10,04 & 4,01 & 1,85 & 3,81 & 1,62 & 1,1 & 4,47 & 27,5 & 12,37 & 2,06 & 1,47 & 0,7 & 2,6180 & $-0,2896$ & 2,5549 \\
\hline & & & 1,52 & 1,64 & 1,29 & 6,23 & 2,49 & 1,95 & 2,62 & 1,08 & 1,2 & 3,04 & 29,6 & 7,96 & 2,20 & 0,89 & 0,7 & 2,5975 & $-0,2737$ & 2,6103 \\
\hline
\end{tabular}


Tabela 38 - Características geométricas da seção transversal dos perfis cartola

\begin{tabular}{|c|c|c|c|c|c|c|c|c|c|c|c|c|c|c|}
\hline $\mathrm{h}$ & B & $\mathrm{e}=\mathrm{r}$ & S & M & $\mathrm{J}_{\mathrm{x}}$ & $\mathrm{W}_{\mathrm{x}}$ & $i_{x}$ & $\mathrm{e}_{\mathrm{y}}$ & $\mathbf{J}_{\mathrm{y}}$ & $\mathrm{W}_{\mathrm{y}}$ & $\mathrm{i}_{\mathrm{y}}$ & \multirow[b]{2}{*}{$\alpha_{x x}$} & \multirow[b]{2}{*}{$\alpha_{x y}$} & \multirow[b]{2}{*}{$\alpha_{\mathrm{yy}}$} \\
\hline $\mathrm{mm}$ & $\mathrm{mm}$ & $\mathrm{mm}$ & $\mathrm{cm}^{2}$ & $\mathrm{~kg} / \mathrm{m}$ & $\mathrm{cm}^{4}$ & $\mathrm{~cm}^{3}$ & $\mathrm{~cm}$ & $\mathrm{~cm}$ & $\mathrm{~cm}^{4}$ & $\mathrm{~cm}^{3}$ & $\mathrm{~cm}$ & & & \\
\hline \multirow[t]{2}{*}{50} & \multirow[t]{2}{*}{50} & 3,04 & 5,54 & 4,35 & 19,16 & 7,34 & 1,85 & 2,30 & 37,84 & 7,56 & 2,61 & 4,7241 & 0,0 & 2,5020 \\
\hline & & 1,90 & 3,59 & 2,81 & 13,48 & 5,24 & 1,93 & 2,43 & 24,94 & 4,98 & 2,63 & 4,9256 & 0,0 & 2,4597 \\
\hline \multirow[t]{2}{*}{50} & \multirow[t]{2}{*}{75} & 3,04 & 6,30 & 4,95 & 22,61 & 7,93 & 1,89 & 2,15 & 80,73 & 12,92 & 3,58 & 3,3821 & 0,0 & 3,0360 \\
\hline & & 1,90 & 4,06 & 3,19 & 15,81 & 5,60 & 1,97 & 2,18 & 52,88 & 8,46 & 3,61 & 3,4932 & 0,0 & 2,9719 \\
\hline \multirow[t]{2}{*}{50} & \multirow[t]{2}{*}{100} & 3,42 & 7,86 & 6,17 & 27,37 & 8,82 & 1,86 & 1,89 & 159,0 & 21,20 & 4,49 & 2,6998 & 0,0 & 3,6863 \\
\hline & & 1,90 & 4,54 & 3,56 & 17,65 & 5,77 & 1,97 & 1,94 & 93,52 & 12,46 & 4,53 & 2,7955 & 0,0 & 3,5683 \\
\hline \multirow[t]{2}{*}{100} & \multirow[t]{2}{*}{50} & 3,04 & 8,58 & 6,73 & 107,28 & 20,93 & 3,53 & 4,87 & 59,24 & 11,84 & 2,62 & 10,7833 & 0,0 & 1,6551 \\
\hline & & 1,90 & 5,49 & 4,31 & 71,78 & 14,13 & 3,61 & 4,92 & 37,74 & 7,54 & 2,62 & 11,2409 & 0,0 & 1,6471 \\
\hline \multirow[t]{2}{*}{100} & \multirow[t]{2}{*}{75} & 3,42 & 10,43 & 8,18 & 135,19 & 24,47 & 3,59 & 4,47 & 142,11 & 22,73 & 3,69 & 6,6852 & 0,0 & 1,8558 \\
\hline & & 1,90 & 5,96 & 4,68 & 82,03 & 15,01 & 3,70 & 4,53 & 80,97 & 12,95 & 3,68 & 6,9745 & 0,0 & 1,8380 \\
\hline \multirow[t]{2}{*}{100} & \multirow[t]{2}{*}{100} & 3,80 & 12,45 & 9,77 & 162,82 & 27,75 & 3,61 & 4,13 & 276,68 & 36,89 & 4,71 & 4,8637 & 0,0 & 2,0917 \\
\hline & & 1,90 & 6,44 & 5,05 & 50,76 & 15,67 & 3,75 & 4,20 & 142,86 & 19,04 & 4,70 & 5,0767 & 0,0 & 2,0606 \\
\hline \multirow[t]{2}{*}{110} & \multirow[t]{2}{*}{50} & 3,42 & 10,26 & 8,05 & 150,18 & 26,61 & 3,82 & 5,35 & 71,22 & 14,24 & 2,63 & 12,1093 & 0,0 & 1,5952 \\
\hline & & 1,90 & 5,87 & 4,60 & 90,92 & 16,29 & 3,93 & 5,42 & 40,30 & 8,06 & 2,62 & 12,8007 & 0,0 & 1,5866 \\
\hline \multirow[t]{2}{*}{110} & \multirow[t]{2}{*}{75} & 3,42 & 11,11 & 8,72 & 171,59 & 28,40 & 3,92 & 4,95 & 152,65 & 24,42 & 3,70 & 7,4823 & 0,0 & 1,7684 \\
\hline & & 1,90 & 6,34 & 4,98 & 103,44 & 17,30 & 4,03 & 5,02 & 86,59 & 13,85 & 3,69 & 7,8053 & 0,0 & 1,7538 \\
\hline \multirow[t]{2}{*}{110} & \multirow[t]{2}{*}{100} & 3,80 & 13,21 & 10,37 & 206,58 & 32,28 & 3,95 & 4,60 & 297,17 & 39,62 & 4,74 & 5,3748 & 0,0 & 1,9746 \\
\hline & & 1,90 & 6,82 & 5,35 & 114,22 & 18,07 & 4,09 & 4,67 & 152,71 & 20,36 & 4,73 & 5,6094 & 0,0 & 1,9491 \\
\hline
\end{tabular}




\subsection{Exemplos de aplicação dos fatores de forma}

Apresentam-se a seguir dois exemplos de aplicação dos fatores de forma dados nas tabelas anteriores:

1) Cálculo da flecha numa viga bi-apoiada cuja seção transversal é um perfil cartola $50 \times 100(\mathrm{~h}=50 \mathrm{~mm}$ e $\mathrm{B}=100 \mathrm{~mm})$ de espessura $3,42 \mathrm{~mm}$. Admite-se para o aço módulo de elasticidade longitudinal $\mathrm{E}=21.000 \mathrm{kN} / \mathrm{cm}^{2}$ e coeficiente de Poisson $v=0,3$ e considera-se flexão em torno do eixo $\mathrm{x}$. $\mathrm{O}$ valor do carregamento aplicado na direção do eixo y e o comprimento da viga são dados na figura 60 .

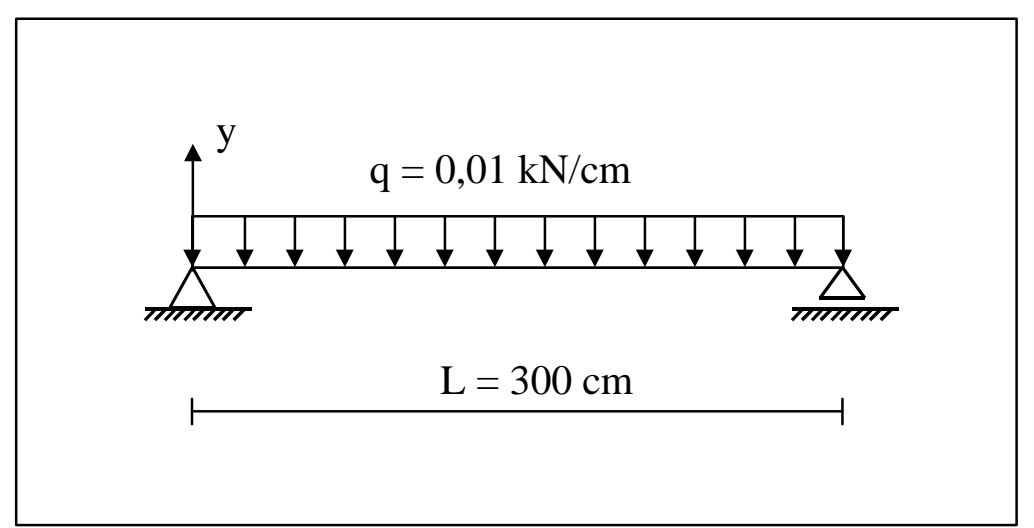

Figura 60 - Viga de aço submetida a força uniformemente distribuída

Segundo a teoria de Euler-Bernoulli, a flecha para o tipo de carregamento e de condições de contorno dados acima é:

$$
\mathrm{f}=\frac{5 \cdot \mathrm{q} \cdot \mathrm{L}^{4}}{384 \cdot \mathrm{E} \cdot \mathrm{I}_{\mathrm{x}}}
$$

Sendo $I_{x}=27,37 \mathrm{~cm}^{4}$, a flecha resultante será:

$$
\mathrm{f}=1,83497 \mathrm{~cm}
$$

Já segundo a teoria de Timoshenko, a flecha, para as mesmas condições, é dada por:

$$
\mathrm{f}=\frac{5 \cdot \mathrm{q} \cdot \mathrm{L}^{4}}{384 \cdot \mathrm{E} \cdot \mathrm{I}_{\mathrm{x}}} \cdot\left(1+\frac{48 \cdot \alpha_{\mathrm{yy}} \cdot \mathrm{E} \cdot \mathrm{I}_{\mathrm{x}}}{5 \cdot \mathrm{G} \cdot \mathrm{A} \cdot \mathrm{L}^{2}}\right)
$$

Sendo $\alpha_{\mathrm{yy}}=3,6863$ o fator de forma da seção transversal para carregamento na direção y e $\mathrm{A}=7,86 \mathrm{~cm}^{2}$ a área da seção transversal, tem-se a flecha: 
$\mathrm{f}=1,84150 \mathrm{~cm}$

2) Cálculo da flecha numa viga engastada de comprimento $300 \mathrm{~cm}$, cuja seção transversal é um perfil $\mathrm{Z}$ simples de dimensões $\mathrm{h}=200 \mathrm{~mm}, \mathrm{~B}=50 \mathrm{~mm}$ e espessura 4,76 mm. Admite-se para o aço módulo de elasticidade longitudinal $\mathrm{E}=21.000 \mathrm{kN} / \mathrm{cm}^{2}$ e coeficiente de Poisson $v=0,3$. Aplica-se uma força concentrada na direção do eixo z, indicada na figura 61. Como a seção é assimétrica haverá deslocamento transversal da barra nas duas direções principais. Para o cálculo dos deslocamentos na extremidade, utiliza-se a matriz dada por PILKEY (1995), apresentada no item 4.4 do capítulo 4.

As características geométricas da seção necessárias à montagem da matriz de rigidez são os momentos de inércia $I_{y}=704,50 \mathrm{~cm}^{4}$ e $I_{z}=17,23 \mathrm{~cm}^{4}$, a área $\mathrm{A}=13,39 \mathrm{~cm}^{4}$ e os fatores de forma $\alpha_{\mathrm{yy}}=5,1580, \alpha_{\mathrm{yz}}=-0,0274$ e $\alpha_{\mathrm{zz}}=1,5866$.

Impõe-se que as duas translações e as duas rotações no engaste sejam nulas e resolve-se o sistema linear resultante. As flechas na extremidade terão como valor:

$$
\begin{aligned}
& \mathrm{f}_{\mathrm{z}}=0,61274 \mathrm{~cm} \\
& \mathrm{f}_{\mathrm{y}}=-7,60056 \cdot 10^{-5} \mathrm{~cm}
\end{aligned}
$$

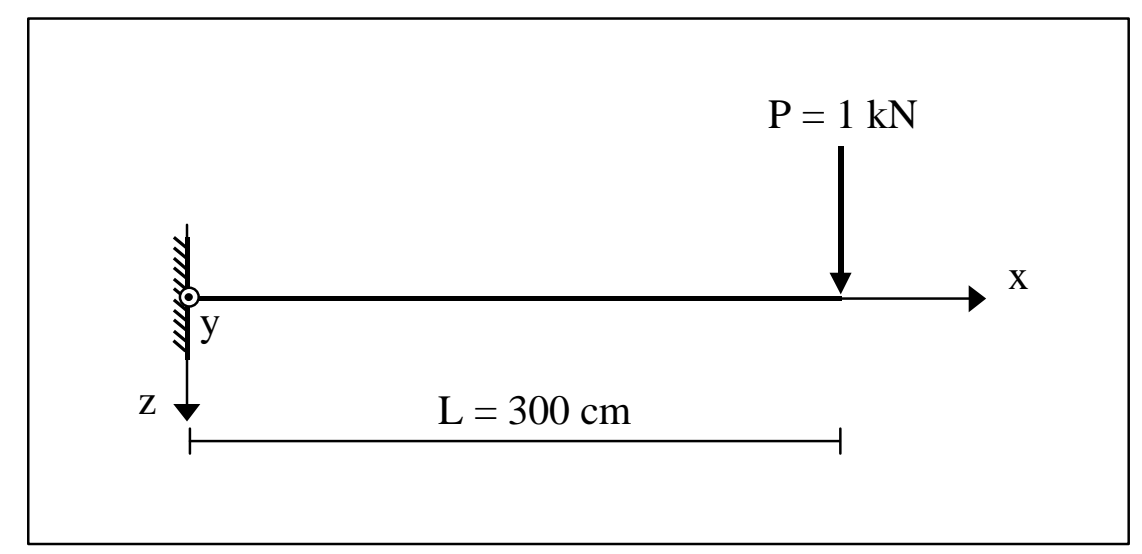

Figura 61 - Viga de aço submetida a força concentrada na extremidade 


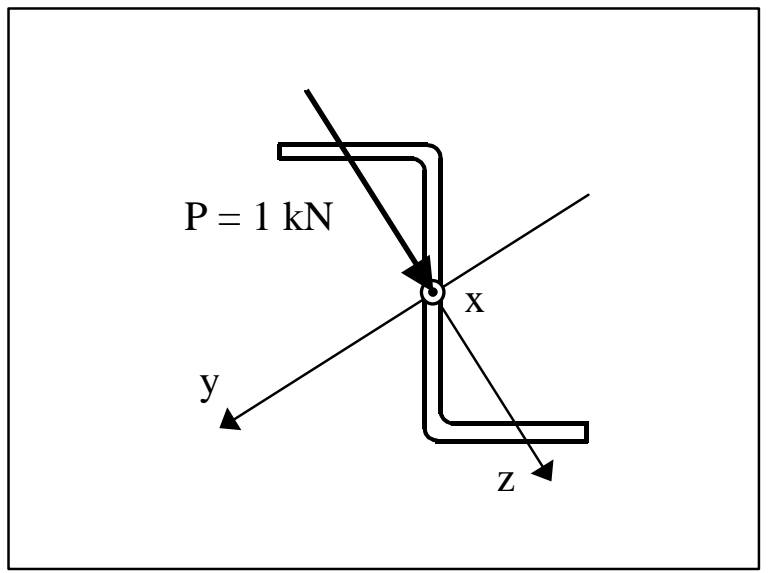

Figura 62 - Seção transversal do perfil Z simples 


\section{8 - CONSIDERAÇÕES FINAIS E SUGESTÕES}

Neste trabalho, incorporou-se a um programa de análise estrutural de edifícios altos já existente (CEASO 01) o recurso de modelar seus elementos verticais de contraventamento (pilares e núcleos resistentes) como barras que seguem a teoria de Timoshenko. A análise dos resultados dos exemplos realizada no capítulo 6 permite que algumas conclusões gerais possam ser estabelecidas quanto ao efeito de considerar a deformação por esforço cortante nos elementos verticais:

- A translação do nó mestre das lajes é sempre aumentada. Nota-se que quando o edifício apresenta dimensões mais próximas às adotadas em projetos (como é o caso do exemplo 5) esse aumento é mais notável apenas nos pavimentos mais baixos, sendo a alteração da flecha lateral muito pequena.

- De uma forma geral, pode-se considerar que haja uma redução de rigidez das estruturas à torção, embora isso não tenha sido confirmado em todos os exemplos. Naqueles exemplos em que a rotação foi aumentada (exemplos 2 e 5), notou-se um aumento muito grande do momento torçor e do bimomento nos núcleos, no primeiro pavimento.

Se fosse considerada nesses elementos a deformação pela tensão de cisalhamento devida ao momento de flexo-torção, haveria uma redução da rigidez tanto à torção quanto ao empenamento e, provavelmente, o aumento destes esforços seria menor.

- O efeito de redistribuição de esforços cortantes entre núcleos e pilares pôde ser constatado nos exemplos 3, 4 e 5, sendo muito pronunciado nos pavimentos inferiores e mínimo quando próximo ao topo do edifício Nos casos em que os núcleos absorvem uma proporção muito grande da força horizontal (próximo a $90 \%$ ), uma redução de seu esforço cortante pode ser proporcionalmente pequena, mas representar muito quando transferida para os pilares (como um acréscimo). No caso do pilar 20 do exemplo 5, o esforço cortante aumentou $354,47 \%$, no primeiro andar. 
Entre as sugestões para trabalhos futuros que possam aperfeiçoar a modelagem de edifícios altos, podem-se citar:

- Considerar a deformação pelo esforço cortante nos pilares e núcleos estruturais numa análise em teoria de $2^{\mathrm{a}}$ ordem.

- Considerar no núcleo as deformações devidas às tensões de cisalhamento derivadas do momento de flexo-torção. A formulação para essa alteração pode ser encontrada em BATOZ (1995), que desenvolveu um elemento baseado na teoria de barra de BENSCOTER (1954). Por esta teoria, mantém-se a definição de área setorial da teoria de Vlassov (que foi deduzida desprezando-se a distorção na seção transversal), porém adota-se uma função de empenamento $\psi$ e não mais a derivada da rotação da barra $\left(\theta_{\mathrm{x}}{ }^{\prime}\right)$. Essa nova função de empenamento irá fazer a consideração da deformação pela tensão de cisalhamento.

- Considerar a rigidez das lajes à flexão, por meio de sua discretização em elementos de placa e considerar sua interação com os núcleos resistentes. A consideração da rigidez das lajes teria, nos núcleos, o efeito de redução do empenamento da seções transversais.

- Pode-se também considerar a laje discretizada como elemento de casca, o que faria com que ela não fosse mais infinitamente rígida em seu plano e fazendo com que não houvesse mais uniformidade de deslocamentos e rotações das extremidades dos elementos verticais numa mesma laje.

- Discretizar as paredes dos núcleos como elementos de casca. Esta abordagem permitiria um maior refinamento da análise e facilitaria o acoplamento das lajes com os núcleos. Além disso, permitiria uma aferição tanto do modelo de pilar de rigidez equivalente (Yagui) como do de barra de sete deslocabilidades (Taranath). Teria, naturalmente, o inconveniente de um custo computacional maior. 


\section{REFERÊNCIAS BIBLIOGRÁFICAS}

ARCHER, J. S. (1965). Consistent matrix formulations for structural analysis using finite element techniques. Journal of the American Institute of Aeronautics and Astronauts, v. 3, p. 1910-1918.

ASSOCIAÇÃO BRASILEIRA DE NORMAS TÉCNICAS (1980). NBR 6355 - Perfis estruturais de aço formados a frio. Rio de Janeiro.

ASSOCIAÇÃO BRASILEIRA DE NORMAS TÉCNICAS (1987). NBR 6123 - Forças devidas ao vento em edificações . Rio de Janeiro.

BARBOSA, J. A. (1978). Edifícios com paredes de seção aberta contraventadas por lintéis, sob carga lateral. São Carlos. 333p. Dissertação (Mestrado) - Escola de Engenharia de São Carlos, Universidade de São Paulo.

BECKER, E. P. (1989). Edifícios altos: interação tridimensional das peças de contraventamento. São Carlos. 181p. Dissertação (Mestrado) - Escola de Engenharia de São Carlos, Universidade de São Paulo.

BENSCOTER, S. U. (1954). A theory of torsion bending for multicell beams. Journal of Applied Mechanics, v. 21, p.25-34.

BICKFORD, W. B. (1982). A consistent higher order beam theory. Developments in Theoretical and Applied Mechanics, v. 11, p. 137-150, Apr.

CARMO, R. M. S. (1995). Efeitos de segunda ordem em edifícios usuais de concreto armado. São Carlos. 112p. Dissertação (Mestrado) - Escola de Engenharia de São Carlos, Universidade de São Paulo.

CARNEGIE, W. ; THOMAS, J. ; DOKUMACI, E. (1969). An improved method of matrix displacement analysis in vibration problems. Aero. Quart. , v. 20, p. 321-332. 
COSTA, J. L. (1982). Núcleos estruturais sobre fundações flexíveis. São Carlos. 187p. Dissertação (Mestrado) - Escola de Engenharia de São Carlos, Universidade de São Paulo.

COWPER, G. R. (1966). The shear coefficient in Timoshenko's beam theory. Journal of Applied Mechanics, v. 33, n. 2, p. 335-340.

DAVIS, R. ; HENSHELL, R. D. ; WARBURTON, G. B. (1972). A Timoshenko beam element. Journal of Sound and Vibration, v. 22, p. 475487.

DONG, S. B. ; WOLF, J. A. (1973). Effect of transverse shear deformation on vibrations of planar structures composed of beam-type elements. Journal of Acoustical Society of America, v. 40, p. 1058-1063.

GERE, J. M. ; WEAVER JR., W. (1965). Analysis of framed structures. Princeton, N. J. , D. Van Nostrand.

HEIDEBRECHT, A. C. ; SWIFT, R. D. (1971). Analysis of asymmetrical coupled shear walls. Journal of the Structural Division, Proc. ASCE, v. 97, n. ST5 , p. 1407-1422, May.

HINDMARCH, A. C. (1983). ODEPACK: a sistematized collection of ODE solvers, in scientific computing. Edited by R. S. Stepleman et al. Amsterdam, North Holland. p. 55-64.

HUGHES, T. J. R. ; TAYLOR, R. L. ; KANOKNUKULCHAI, W. (1977). A simple and efficient element for plate bending. International Journal for Numerical Methods Engineering, v. 11, p. 1529-1543.

KAPUR, K. K. (1966). Vibrations of a Timoshenko beam using a finite element approach. Journal of the Acoustical Society of America, v. 40, p.1058-1063.

LEVINSON, M. (1981). A new rectangular beam theory. Journal of Sound and Vibration, v. 74, p. 81-87.

MASON, W. E. ; HERRMAN, L. R. (1968). Elastic shear analysis of general prismatic beams. Journal of Engineering Mechanics Division, Proc. ASCE, v. 94, n. 4, p. 965-983.

MATIAS JR., I. G. (1997). Análise não linear de estruturas tridimensionais de edifícios altos com núcleos resistentes sobre fundações flexíveis. São 
Carlos. 279p. Dissertação (Mestrado) - Escola de Engenharia de São Carlos, Universidade de São Paulo.

MORI, D. D. (1992). Os núcleos estruturais e a não linearidade geométrica na análise de estruturas tridimensionais de edifícios altos. São Carlos. 195p. Tese (Doutorado) - Escola de Engenharia de São Carlos, Universidade de São Paulo.

NICKEL, R. E. ; SECOR, G. A. (1972). Convergence of consistently derived Timoshenko beam finite elements. International Journal for Numerical Methods in Engineering, v. 5, p. 243-253.

PEREIRA, G. S. (1997). Contribuições à análise de estruturas de contraventamento de edifícios em concreto armado. São Carlos. 97p. Dissertação (Mestrado) - Escola de Engenharia de São Carlos, Universidade de São Paulo.

PETROLITO, J. (1995). Stiffness analysis of beams using a higher-order theory. Computers \& Structures, v. 55, n. 1, p. 33-39.

PILKEY, W.D. ; KANG, W. ; SCHRAMM, U. (1995). New structural matrices for a beam element with shear deformation. Finite Elements in Analysis \& Design, v. 19, p. 25-44.

PLAIS, W. (1998). Análise não-linear de estruturas de barras via elementos finitos utilizando uma teoria de alta-ordem. Belo Horizonte. 125p. Dissertação (Mestrado) - Escola de Engenharia da UFMG, Universidade Federal de Minas Gerais.

PRZEMIENIECKI, J. S. (1968). Theory of matrix structural analysis. New York, McGraw-Hill.

REDDY, J. N. ; HEYLIGER, P. R. (1988). A higher order beam finite element fo bending and vibration problems. Journal of Sound and Vibration, v. 126, n. 2, p. 309-326.

RIBEIRO, S. R. S. (1987). Associação tridimensional de pórticos e paredes de seção aberta em estruturas de edifícios altos. São Carlos. 224 p. Dissertação (Mestrado) - Escola de Engenharia de São Carlos, Universidade de São Paulo. 
SCHRAMM, U. ; KITIS, L. ; KANG, W. ; PILKEY, W. D. (1994). On the shear coefficient in beam theory. Finite Elements in Analysis \& Design, V.16, p. 141-162.

SERRA, J. L. F. A. (1994). Contribuição ao estudo de núcleos resistentes de concreto armado. São Carlos. 128p. Tese (Doutorado) - Escola de Engenharia de São Carlos, Universidade de São Paulo.

SEVERN, R. T. (1970). Inclusion of shear deformation in the stiffness matrix for a beam element. Journal of Strain Analysis, v. 5, p. 239-241.

SHAKOURZADEH, H. ; GUO, Y. Q. ; BATOZ, J. L. (1995). A torsion bending element for thin-walled beams with open and closed cross sections. Computers \& Structures, v. 55, n. 6, p. 1045-1054.

SILVA, R. M. (1989). Análise de estruturas tridimensionais de edifícios altos com núcleos resistentes considerando o efeito $P$ - $\delta$. São Carlos. 239p. Dissertação (Mestrado) - Escola de Engenharia de São Carlos, Universidade de São Paulo.

SURANA, K. S. (1979). Isoparametric elements for cross sectional properties and stress analysis of beams. International Journal for Numerical Methods in Engineering, v. 14, p. 475-497.

TARANATH, B. S. (1968). Torsional behaviour of open section shear wall structures. Southampton, England, University of Southampton.

TESSLER, A. (1991). A two-node beam element including transverse shear and transverse normal deformations. International Journal for Numerical Methods in Engineering, v. 32, p. 1027-1039.

TESSLER, A. ; DONG, S. B. (1981). On a hierarchy of conforming Timoshenko beam elements. Computers \& Structures, v. 14, n. 3-4, p.335-344.

THOMAS, D. L. ; WILSON, J. M. ; WILSON, R. R. (1973). Timoshenko beam finite elements. Journal of Sound and Vibration, v. 31, p. 315-330.

THOMAS, J. ; ABBAS, B. A. H. (1975). Finite element model for dynamic analysis of Timoshenko beam. Journal of Sound and Vibration, v. 41, n.3, p. 291-299. 
TIMOSHENKO, S. P. (1921). On the correction for shear of the differential equation for transverse vibrations of prismatic bars. Philosophical Magazine, v. 41, p. 744-746.

TSO, W. K. ; BISWAS, J. K. (1973). General analysis of nonplanar coupled shear walls. Journal of the Structural Division, v. 99, n. 3, p. 365-380, Mar.

VLASSOV, B. P. (1962). Pièces longues en voiles minces. Trad. por G. Smirnoff. 2. ed. Paris, Eyrolles.

YAGUI, T. (1971). Estruturas constituídas de paredes delgadas com diafragmas transversais. São Carlos. 138p. Tese (Doutorado) - Escola de Engenharia de São Carlos, Universidade de São Paulo. 


\section{BIBLIOGRAFIA COMPLEMENTAR}

BATHE, K. J. (1996). Finite element procedures. New Jersey, Prentice Hall.

LOVE, A. E. H. (1944). A treatise on the mathematical theory of elasticity. 4.ed. New York, Dover Publications.

MORI, D. D. (1988). Flexo-torção: barras com seção transversal aberta e paredes delgadas. São Carlos, Escola de Engenharia de São Carlos, Universidade de São Paulo. 132p.

MURRAY, N. W. (1986). Introduction to the theory of thin-walled structures. Oxford, Clarendon Press.

OÑATE, E. (1992). Cálculo de estructuras por el método de elementos finitos. Análisis elástico lineal. Barcelona, Centro Internacional de Métodos Numéricos en Ingeniería.

PILKEY, W. D. ; WUNDERLICH, W. (1992). Mechanics of structures: variational and computational methods. Boca Raton, CRC Press.

REDDY, J. N. (1984). Energy and variational methods in applied mechanics. New York, John Wiley.

SAVASSI, W. (1996). Introdução ao método dos elementos finitos: em análise linear de estruturas. São Carlos, EESC.

TIMOSHENKO, S. P. (1922). On the transverse vibrations of bars of uniform cross section. Philosophical Magazine, v. 43, p. 125-131.

TIMOSHENKO, S. P. ; GERE, J. M. (1961). Theory of elastic stability.

2. ed. New York, McGraw-Hill.

TIMOSHENKO, S. P. (1966). Resistência dos materiais. Trad. por Domício Falcão Moreira. Rio de Janeiro, Ao Livro Técnico.

TIMOSHENKO, S. P. ; GOODIER, J. N. (1980). Teoria da elasticidade. 3. ed. Rio de Janeiro, Guanabara Dois.

ZIENKIEWICZ, O. C. ; TAYLOR, L. R. (1991). The finite element method. 4. ed. London, McGraw-Hill. 